FLOW-INDUCED NOISE OF PERFORATED PLATES AT OBLIQUE ANGLES OF INCIDENCE 


\section{FLOW-INDUCED NOISE OF PERFORATED PLATES AT OBLIQUE ANGLES OF INCIDENCE}

By

PAUL VANOOSTVEEN, B.ENG.MGT.

A Thesis Submitted to the School of Graduate Studies

in Partial Fulfilment of the Requirements

for the Degree

Master of Applied Science

McMaster University

CCopyright by Paul Vanoostveen, October 2017 
Master of Applied Science (2017)

Mechanical Engineering
McMaster University

Hamilton, Ontario, Canada
TITLE:

AUTHOR:

SUPERVISOR:

NUMBER OF PAGES: $\quad$ xxvi, 151

Flow-Induced Noise of Perforated Plates at Oblique Angles of Incidence

Paul Vanoostveen, B.Eng.Mgt. (McMaster University)

Dr. Samir Ziada 


\section{ABSTRACT}

In this thesis, the tonal noise produced by flow over perforated plates at oblique angles of incidence is studied experimentally. A two-dimensional model of a perforated plate is used, where the circular holes of a typical perforated plate are replaced by a series of long rectangular Aluminum slats with an adjustable gap width between them. The slats are $3.175 \mathrm{~mm}$ thick and the gap width between them is set to $3.175 \mathrm{~mm}, 6.35 \mathrm{~mm}$, and 12.7 $\mathrm{mm}$. This simplified model is mounted at the exit of an open-loop wind tunnel and tested at angles of incidence of $0^{\circ}$ to $40^{\circ}$ and flow velocities of 0 to $30 \mathrm{~m} / \mathrm{s}$. An angle of $0^{\circ}$ is defined

as flow parallel to the plate. The acoustic response is studied using microphone measurements, and flow visualization is done using particle image velocimetry. The effect of the angle of incidence, flow velocity, gap width, and streamwise position are investigated.

The flow visualization reveals that tonal noise is produced by the periodic shedding and impingement of vortices at the trailing edge of the gaps. Vortices form in the unstable free shear layer originating at the leading edge of the gap and impinge on the downstream side of the gap. At the downstream corner, these vortices separate into vortex pairs, consisting of one positively rotating and one negatively rotating vortex. These vortices are shed periodically, leading to the production of tonal noise at the shedding frequency.

The effect of the angle of incidence is investigated by changing the angle of the plate with respect to the flow. For a given gap width, tones are produced only for a specific range of angles. Depending on the plate geometry, this range of angles is typically around 
$5^{\circ}$ to $30^{\circ}$. Within this range of angles, the free shear layer impinges on the downstream side of the gap. For angles which are too small or too large, the free shear layer misses this downstream side and tones are not produced. For a larger gap width, tones are produced at smaller angles of incidence.

Similarly, for a given plate geometry, there is a preferred range of flow velocities at which tonal noise is produced. The velocity at which the free shear layer is the most unstable at the tone frequency produces the strongest vortices and the loudest tones. The optimal velocity is lower for larger gap widths.

Finally, it is found that the magnitude of the produced tones increases in the streamwise direction over repeated gaps along the length of the plate. This is due to the local flow conditions changing in the streamwise direction, only reaching the optimal conditions after a certain length of the plate. 


\section{ACKNOWLEDGEMENTS}

I would like to thank my supervisor, Dr. Samir Ziada, for his help and support throughout the course of this research project. Without his guidance and expertise, the work presented in this thesis would not have been possible.

I would also like to thank my fellow members of the Flow-Induced Vibrations and Aeroacoustics research group at McMaster University. Their experience and suggestions proved invaluable during the completion of this work.

I would like to acknowledge Kevin Sia and Adam Royce for their help in manufacturing the experimental apparatus as part of their mechanical engineering capstone project. Finally, I would like to thank the Mechanical Engineering technicians at McMaster University for their technical support in manufacturing and troubleshooting the experimental apparatus used in this project. 


\section{TABLE OF CONTENTS}

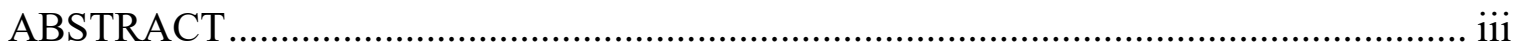

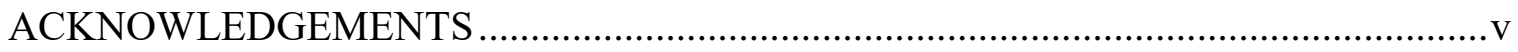

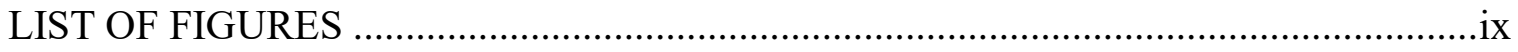

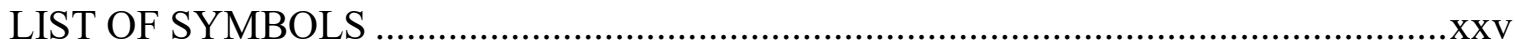

CHAPTER 1: INTRODUCTION ....................................................................... 1

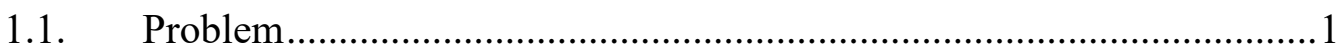

1.2. Thesis Outline ........................................................................

CHAPTER 2: LITERATURE REVIEW …............................................................

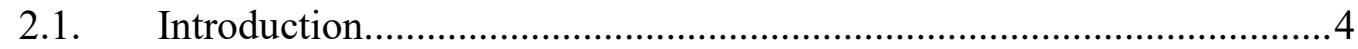

2.2. Shear Layer Instability ...........................................................

2.3. Impinging Shear Flows .......................................................

2.4. Flow Over Perforated Plates .................................................... 12

2.5. 2D Simplifications for Circular Cavities ....................................... 18

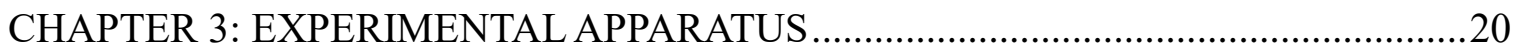

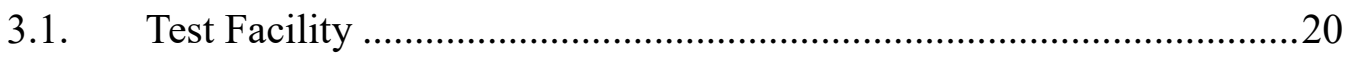

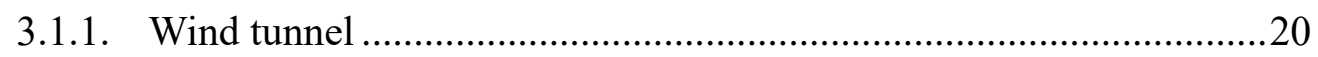

3.1.2. Test section .............................................................................22 


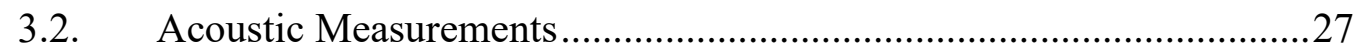

3.3. Particle Image Velocimetry Measurements .......................................29

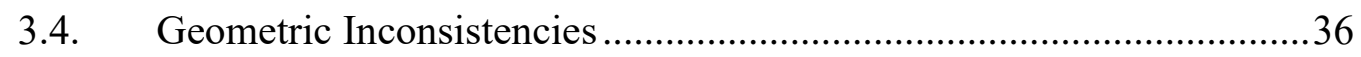

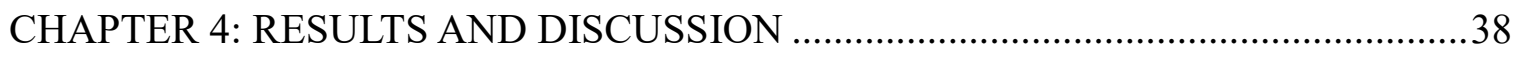

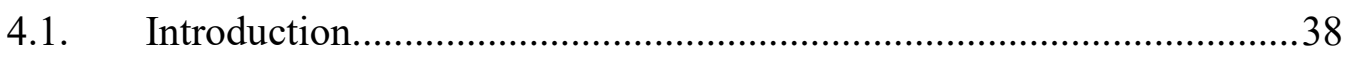

4.2. $\quad$ Acoustic Results - Perforated Plate......................................................38

4.3. Acoustic Results - 2D Model ...............................................................

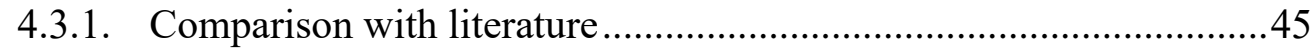

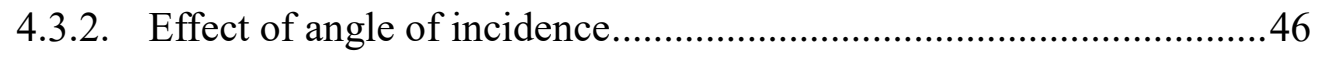

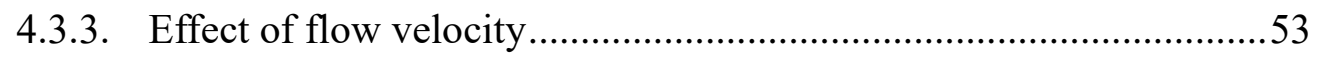

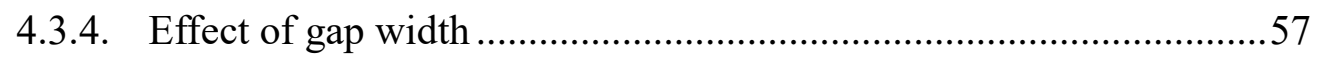

4.3.5. Effect of streamwise position .........................................................58

4.4. Particle Image Velocimetry Results ......................................................63

4.4.1. Laser positioned in front of slats ......................................................63

4.4.2. Laser positioned behind slats .......................................................67

4.4.2.1. Effect of angle of incidence.......................................................

4.4.2.2. Effect of flow velocity ..............................................................

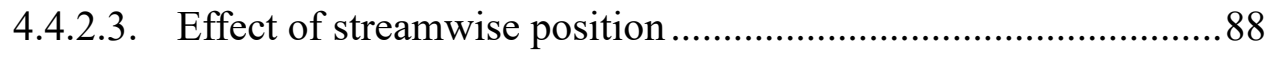




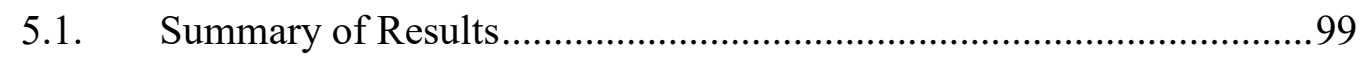

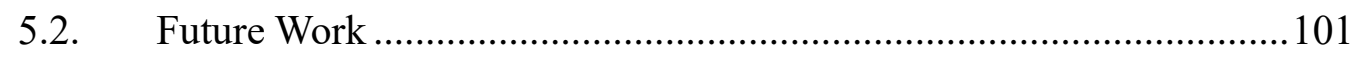

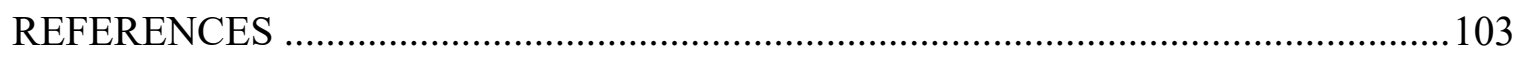

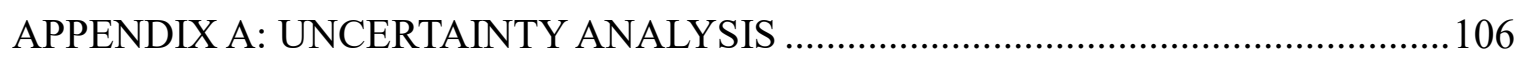

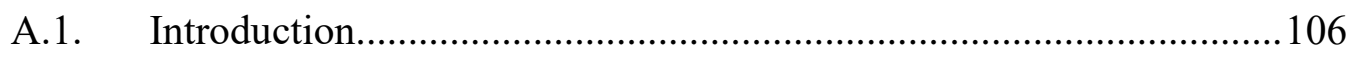

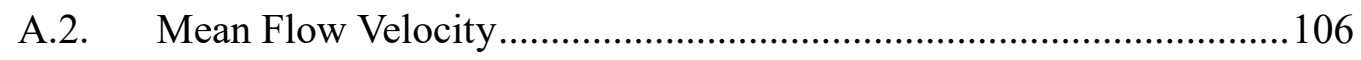

A.3. Acoustic Measurements....................................................................107

A.4. Calculated Values..............................................................................108

A.5. Particle Image Velocimetry Measurements ……................................110

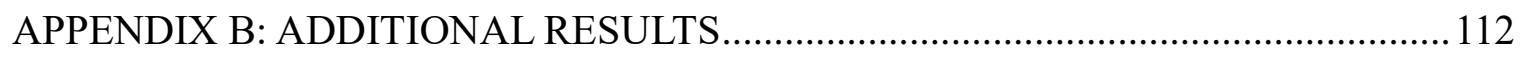

B.1. Acoustic Results - Perforated Plate..................................................... 112

B.2. Acoustic Results - 2D Model .............................................................121

B.2.1. Effect of flow velocity, gap width $=3.175 \mathrm{~mm} \ldots \ldots \ldots \ldots \ldots \ldots \ldots \ldots \ldots . . .121$

B.2.2. Effect of flow velocity, gap width $=6.35 \mathrm{~mm} \ldots \ldots \ldots \ldots \ldots \ldots \ldots \ldots \ldots \ldots . . .126$

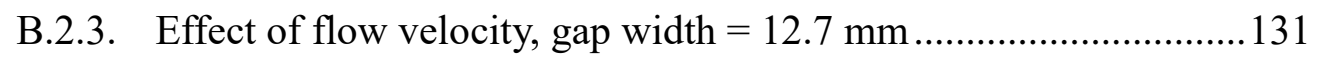

B.2.4. Effect of streamwise position .....................................................136

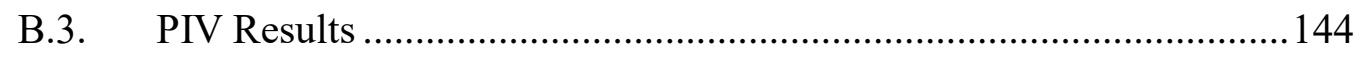

B.3.1. Effect of angle of incidence …………..........................................144 


\section{LIST OF FIGURES}

Figure 1: Perforated metal plates used on the balcony of a building (Schafer, 2017).........1

Figure 2: Dependence of the growth rate of disturbances on the amplitude of excitation for a circular jet with $D=7.5 \mathrm{~cm}, U_{0}=8 \mathrm{~m} / \mathrm{s}, \operatorname{Re}_{\Theta}=122: \circ, 70 \mathrm{~dB} ; \bullet, 80 \mathrm{~dB}$;

$\Delta, 90 \mathrm{~dB} ; \mathrm{x}, 100 \mathrm{~dB}$ (Freymuth, 1966).

Figure 3: Dependence of the growth rate of disturbances on the Strouhal number for a circular jet with $D=7.5 \mathrm{~cm}, U_{0}=16 \mathrm{~m} / \mathrm{s}: \mathbf{\mathbf { X }}, S t_{\Theta}=0.0020 ; \otimes, S t_{\Theta}=0.0040$; -, $S t_{\Theta}=0.0050 ;$ *, $S t_{\Theta}=0.0070 ; \Delta, S t_{\Theta}=0.0080 ; \circ, S t_{\Theta}=0.0090 ; \mathrm{x}, S t_{\Theta}=$ $0.0100 ; \square, S t_{\Theta}=0.0118 ; \boldsymbol{\Lambda}, S t_{\Theta}=0.0148 ; \bullet, S t_{\Theta}=0.0176 ; \mathbf{\square}, S t_{\Theta}=0.0234$ (Freymuth, 1966).

Figure 4: Growth rate of disturbances as a function of Strouhal number based on momentum thickness, $S t_{\Theta}$. The lines are the numerical results of Michalke for both spatial and temporal amplification of disturbances; and the symbols are the experimental results of Freymuth: O, round jet; X, planar jet (Michalke, 1965; Freymuth, 1966).

Figure 5: Fluid-dynamic mechanism caused by flow impingement on an edge; and the frequency variation with (a) varying free-stream velocity $U$ and constant impingement length $L$, and (b) varying impingement length $L$ and constant free-stream velocity $U$ (Knisely \& Rockwell, 1982).

Figure 6: Basic configurations of shear-layer and impingement-edge geometries that produce self-sustained oscillations (Rockwell \& Naudascher, 1979).

Figure 7: Flow over an orifice with (a) perpendicular angle of incidence, (b) parallel angle of incidence, (c) oblique angle of incidence. 
Figure 8: Geometric parameters of interest for a perforated plate.

Figure 9: Results of Feng for an angle of incidence of $15^{\circ}$ and a velocity of $15 \mathrm{~m} / \mathrm{s}$ for a hole diameter of $20 \mathrm{~mm}$ (plate 1), $12 \mathrm{~mm}$ (plate 2), $11 \mathrm{~mm}$ (plate 4), $10 \mathrm{~mm}$ (plate 5), and $8 \mathrm{~mm}$ (plate 3) (Feng, 2012).

Figure 10: Results of Feng for an angle of incidence of $15^{\circ}$, a hole diameter of $8 \mathrm{~mm}$ (plate 3) and variable velocity (Feng, 2012). 16

Figure 11: Acoustic results of Blinet et al. (2015) for a hole diameter of $20 \mathrm{~mm}$, an angle of incidence of $10^{\circ}$ and a velocity of $15 \mathrm{~m} / \mathrm{s}$. The red line is the result with the blunt edge of the plate exposed to the flow, and the blue line is the result with the sharp edge of the plate exposed to the flow.

Figure 12: Test section consisting of a frame with rectangular slats mounted on hinges at the exit of an open-loop wind tunnel.

Figure 13: Horizontal cross-sectional view of the test section. .25

Figure 14: FFT of the microphone signal showing the limits of the bandpass filter centered at the dominant tonal frequency.

Figure 15: Time signal of the unfiltered and filtered microphone signal showing the trigger location. The red X's denote the 8 points in the acoustic cycle at which PIV images are taken: $0^{\circ}, 45^{\circ}, 90^{\circ}, 125^{\circ}, 180^{\circ}, 225^{\circ}, 270^{\circ}$, and $315^{\circ}$.

Figure 16: Cross-sectional view of a series of two slats: (a) correctly aligned slats, (b) upstream slat twisted, (c) upstream slat bent causing offset. 
Figure 17: Frequency spectra for a perforated plate with an angle of incidence of $15^{\circ}$ and a flow velocity of $15 \mathrm{~m} / \mathrm{s}$. The black lines are the results of Feng for a hole diameter of $8 \mathrm{~mm}$ to $20 \mathrm{~mm}(\mathrm{D} / \mathrm{t}=5.3$ to 13.3$)$, expressed in A-weighted sound power level (dB) (Feng, 2012). The colored lines are the results of this research, with a hole diameter of $6.35 \mathrm{~mm}(D / t=4)$, expressed in A-weighted sound pressure level $(\mathrm{dB})$. The blue line is for the blunt edges exposed to the flow and the red line is for the sharp edges exposed to the flow.

Figure 18: Influence of the wind velocity on the frequency spectrum for a perforated plate at an angle of incidence of $15^{\circ}$. The black lines are the results of Feng for a hole diameter of $8 \mathrm{~mm}(\mathrm{D} / \mathrm{t}=5.3)$, expressed in A-weighted sound power level (dB) (Feng, 2012). The colored lines are the results of this research with a hole diameter of $6.35 \mathrm{~mm}(D / t=4)$, expressed in A-weighted sound pressure level (dB). The blunt edges of the plate are exposed to the flow.

Figure 19: Frequency spectra for a perforated plate with a hole diameter of $6.35 \mathrm{~mm}$, an angle of incidence of 15 degrees, and a flow velocity of 5 to $30 \mathrm{~m} / \mathrm{s}$. The red line is for the sharp edge of the perforations exposed to the flow, while the blue line is for the blunt edge exposed to the flow.

Figure 20: Frequency spectra for a perforated plate with a hole diameter of $6.35 \mathrm{~mm}$, a flow velocity of $20 \mathrm{~m} / \mathrm{s}$, and an angle of incidence of 0 to 40 degrees. The red line is for the sharp edge of the perforations exposed to the flow, while the blue line is for the blunt edge exposed to the flow. 
Figure 21: Frequency spectra for an angle of incidence of $15^{\circ}$ and a flow velocity of 15 $\mathrm{m} / \mathrm{s}$. The black lines are the results of Feng for a perforated plate with a hole diameter of $8 \mathrm{~mm}$ to $20 \mathrm{~mm}(\mathrm{D} / \mathrm{t}=5.3$ to 13.3$)$, expressed in A-weighted sound power level (dB) (Feng, 2012). The red and blue lines are the results of this research, for a perforated plate with a hole diameter of $6.35 \mathrm{~mm}(D / t=4)$, expressed in A-weighted sound pressure level $(\mathrm{dB})$. The Green line is the results of this research, for the rectangular slats with a gap width of $6.35 \mathrm{~mm}$ $(H / t=2)$.

Figure 22: Frequency spectra for a gap width of $6.35 \mathrm{~mm}$, a flow velocity of $20 \mathrm{~m} / \mathrm{s}$, and an angle of incidence of 0 to 40 degrees. The Strouhal number, St, is based on the effective gap width, $d_{e}$. This definition of $S t$ is used in all the analysis to follow.

Figure 23: This illustration shows the angles of incidence favorable to the production of tonal noise for a gap width, $H=6.35 \mathrm{~mm}(0.25$ ") and an angle of incidence in the range $0^{\circ} \leq \theta \leq 45^{\circ}$. Green indicates that tones are produced, while red indicates that tones are not produced. Tones are produced for $\theta=5^{\circ}$ to $\theta=30^{\circ}$.

Figure 24: Frequency spectra for a gap width of $12.7 \mathrm{~mm}$, a flow velocity of $20 \mathrm{~m} / \mathrm{s}$, and an angle of incidence of 0 to 40 degrees. .50

Figure 25: This illustration shows the angles of incidence favorable to the production of tonal noise for a gap width, $H=12.7 \mathrm{~mm}(0.5$ ") and an angle of incidence in the range $0^{\circ} \leq \theta \leq 45^{\circ}$. Green indicates that tones are produced, while red indicates that tones are not produced. Tones are produced for $\theta=5^{\circ}$ to $\theta=25^{\circ}$.

Figure 26: Frequency spectra for a gap width of $3.175 \mathrm{~mm}$, a flow velocity of $20 \mathrm{~m} / \mathrm{s}$, and an angle of incidence of 0 to 40 degrees. 
Figure 27: This illustration shows the angles of incidence favorable to the production of tonal noise for a gap width, $H=3.175 \mathrm{~mm}(0.125$ ") and an angle of incidence in the range $0^{\circ} \leq \theta \leq 45^{\circ}$. Green indicates that tones are produced, while red indicates that tones are not produced. Tones are produced for $\theta=15^{\circ}$ to $\theta=$ $45^{\circ}$

Figure 28: This illustration shows the three classes of inlet flows possible for the slats. In arrow \#1, the angle of incidence is too small to produce tones. In arrow \#3, the angle of incidence is too large to produce tones. In arrow \#2, the flow impinges on the downstream side of the gap and tones may be produced.......52

Figure 29: Frequency spectra for a gap width of $3.175 \mathrm{~mm}$, an angle of incidence of 30 degrees, and a flow velocity of 5 to $30 \mathrm{~m} / \mathrm{s}$.

Figure 30: Frequency spectra for a gap width of $6.35 \mathrm{~mm}$, an angle of incidence of 15 degrees, and a flow velocity of 5 to $30 \mathrm{~m} / \mathrm{s}$.

Figure 31: Frequency spectra for a gap width of $12.7 \mathrm{~mm}$, an angle of incidence of 15 degrees, and a flow velocity of 5 to $30 \mathrm{~m} / \mathrm{s}$.

Figure 32: Frequency spectra for a gap width of $3.175 \mathrm{~mm}\left(1 / 8^{\prime \prime}\right), 6.35 \mathrm{~mm}(1 / 4 ")$, and $12.7 \mathrm{~mm}(1 / 2 ")$, a flow velocity of $20 \mathrm{~m} / \mathrm{s}$, and an angle of incidence of 0 to 40 degrees.

Figure 33: Frequency spectra for a gap width of $6.35 \mathrm{~mm}$, an angle of incidence of $15^{\circ}$, and a flow velocity of $20 \mathrm{~m} / \mathrm{s}$. Results are shown for un-taped (blue \& red) and taped (green \& pink) slats. The streamwise position is varied, from before the first gap to after the last gap. For Case \#4, spectra are only shown for the $3^{\text {rd }}$ to $24^{\text {th }}$ gaps. .60 
Figure 34: Overall sound pressure level for a gap width of $6.35 \mathrm{~mm}$, an angle of incidence of $15^{\circ}$, and a flow velocity of $20 \mathrm{~m} / \mathrm{s}$. Results are shown for un-taped (blue \& red) and taped (green $\&$ pink) slats. The streamwise position is varied, from before the first gap to after the last gap.

Figure 35: PIV results showing the phase-locked velocity field at the 24th gap for a gap width of $6.35 \mathrm{~mm}$, an angle of incidence of 15 degrees, and a flow velocity of $20 \mathrm{~m} / \mathrm{s}$. Images are taken with the laser on the front side of the slats.

Figure 36: PIV results showing the phase-locked vorticity field at the 24th gap for a gap width of $6.35 \mathrm{~mm}$, an angle of incidence of 15 degrees, and a flow velocity of $20 \mathrm{~m} / \mathrm{s}$. Images are taken with the laser on the front side of the slats.

Figure 37: PIV results showing the phase-locked velocity field at the 24th gap for a gap width of $6.35 \mathrm{~mm}$, an angle of incidence of 15 degrees, and a flow velocity of $20 \mathrm{~m} / \mathrm{s}$. Images are taken with the laser on the back side of the slats.

Figure 38: PIV results showing the phase-locked vorticity field at the 24th gap for a gap width of $6.35 \mathrm{~mm}$, an angle of incidence of 15 degrees, and a flow velocity of $20 \mathrm{~m} / \mathrm{s}$. Images are taken with the laser on the back side of the slats.

Figure 39: PIV results showing the average velocity field at the 24th gap for a gap width of $6.35 \mathrm{~mm}$, an angle of incidence of 0 degrees, and a flow velocity of $20 \mathrm{~m} / \mathrm{s}$.

Figure 40: PIV results showing the average vorticity field at the 24th gap for a gap width of $6.35 \mathrm{~mm}$, an angle of incidence of 0 degrees, and a flow velocity of $20 \mathrm{~m} / \mathrm{s}$.

Figure 41: PIV results showing the average velocity field at the 24th gap for a gap width of $6.35 \mathrm{~mm}$, an angle of incidence of 35 degrees, and a flow velocity of $20 \mathrm{~m} / \mathrm{s}$. 
Figure 42: PIV results showing the average vorticity field at the 24th gap for a gap width of $6.35 \mathrm{~mm}$, an angle of incidence of 35 degrees, and a flow velocity of $20 \mathrm{~m} / \mathrm{s}$.

Figure 43: PIV results showing the average velocity (left) and vorticity (right) fields at the 24th gap for a gap width of $6.35 \mathrm{~mm}$ and a flow velocity of $20 \mathrm{~m} / \mathrm{s}$. The angle of incidence is $0^{\circ}$ for the top row, $15^{\circ}$ for the middle row, and $35^{\circ}$ for the bottom row. The white lines are streamlines.

Figure 44: PIV results showing the average velocity field at the 24th gap for a gap width of $6.35 \mathrm{~mm}$, an angle of incidence of 15 degrees, and a flow velocity of $10 \mathrm{~m} / \mathrm{s}$.

Figure 45: PIV results showing the average vorticity field at the 24th gap for a gap width of $6.35 \mathrm{~mm}$, an angle of incidence of 15 degrees, and a flow velocity of $10 \mathrm{~m} / \mathrm{s}$.

Figure 46: PIV results showing the phase-locked velocity field at the 24th gap for a gap width of $6.35 \mathrm{~mm}$, an angle of incidence of 15 degrees, and a flow velocity of $15 \mathrm{~m} / \mathrm{s}$

Figure 47: PIV results showing the phase-locked vorticity field at the 24th gap for a gap width of $6.35 \mathrm{~mm}$, an angle of incidence of 15 degrees, and a flow velocity of $15 \mathrm{~m} / \mathrm{s}$ .78

Figure 48: PIV results showing the phase-locked velocity field at the 24th gap for a gap width of $6.35 \mathrm{~mm}$, an angle of incidence of 15 degrees, and a flow velocity of $25 \mathrm{~m} / \mathrm{s}$

Figure 49: PIV results showing the phase-locked vorticity field at the 24th gap for a gap width of $6.35 \mathrm{~mm}$, an angle of incidence of 15 degrees, and a flow velocity of $25 \mathrm{~m} / \mathrm{s}$. 
Figure 50: PIV results showing the phase-locked velocity field at the 24th gap for a gap width of $6.35 \mathrm{~mm}$, an angle of incidence of 15 degrees, and a flow velocity of $30 \mathrm{~m} / \mathrm{s}$

Figure 51: PIV results showing the phase-locked vorticity field at the 24th gap for a gap width of $6.35 \mathrm{~mm}$, an angle of incidence of 15 degrees, and a flow velocity of $30 \mathrm{~m} / \mathrm{s}$ .82

Figure 52: PIV results showing the average velocity (left) and vorticity (right) fields at the 24th gap for a gap width of $6.35 \mathrm{~mm}$, an angle of incidence of 15 degrees, and a flow velocity of 10 to $30 \mathrm{~m} / \mathrm{s}$. The white lines are streamlines. .83

Figure 53: PIV results showing the phase-locked vorticity field at a single phase at the $24^{\text {th }}$ gap for a gap width of $6.35 \mathrm{~mm}$, an angle of incidence of 15 degrees, and a flow velocity of 15 to $30 \mathrm{~m} / \mathrm{s}$. .85

Figure 54: Strouhal number based on momentum thickness, Ste, as a function of $\mathrm{X}^{*}$. Results are shown for the $24^{\text {th }}$ gap for a gap width of $6.35 \mathrm{~mm}$, an angle of incidence of 15 degrees, and a flow velocity of 10 to $30 \mathrm{~m} / \mathrm{s}$. The region between the theoretical limits of instability are values of $\mathrm{St}_{\Theta}$ at which the free shear layer is most unstable, based on the work of Michalke (1972).

Figure 55: PIV results showing the phase-locked velocity field at the 18th gap for a gap width of $6.35 \mathrm{~mm}$, an angle of incidence of 15 degrees, and a flow velocity of $20 \mathrm{~m} / \mathrm{s}$

Figure 56: PIV results showing the phase-locked vorticity field at the 18th gap for a gap width of $6.35 \mathrm{~mm}$, an angle of incidence of 15 degrees, and a flow velocity of $20 \mathrm{~m} / \mathrm{s}$ .90 
Figure 57: PIV results showing the phase-locked velocity field at the 12th gap for a gap width of $6.35 \mathrm{~mm}$, an angle of incidence of 15 degrees, and a flow velocity of $20 \mathrm{~m} / \mathrm{s}$

Figure 58: PIV results showing the phase-locked vorticity field at the 12th gap for a gap width of $6.35 \mathrm{~mm}$, an angle of incidence of 15 degrees, and a flow velocity of $20 \mathrm{~m} / \mathrm{s}$ .92

Figure 59: PIV results showing phase-locked velocity field at the 6th gap for a gap width of $6.35 \mathrm{~mm}$, an angle of incidence of 15 degrees, and a flow velocity of $20 \mathrm{~m} / \mathrm{s}$.

Figure 60: PIV results showing the phase-locked vorticity field at the 6th gap for a gap width of $6.35 \mathrm{~mm}$, an angle of incidence of 15 degrees, and a flow velocity of $20 \mathrm{~m} / \mathrm{s}$.

Figure 61: PIV results showing the average velocity (left) and vorticity (right) fields for a gap width of $6.35 \mathrm{~mm}$, an angle of incidence of 15 degrees, and a flow velocity of $20 \mathrm{~m} / \mathrm{s}$ at the $6^{\text {th }}, 12^{\text {th }}, 18^{\text {th }}$, and $24^{\text {th }}$ gap locations. The white lines are streamlines. .95

Figure 62: PIV results showing the phase-locked vorticity field at a single phase at the for a gap width of $6.35 \mathrm{~mm}$, an angle of incidence of 15 degrees, and a flow velocity of $20 \mathrm{~m} / \mathrm{s}$, at the $6^{\text {th }}, 12^{\text {th }}, 18^{\text {th }}$, and $24^{\text {th }}$ gap locations.

Figure 63: Frequency spectra for a perforated plate with a hole diameter of $6.35 \mathrm{~mm}$, an angle of incidence of 0 degrees, and a flow velocity of 5 to $30 \mathrm{~m} / \mathrm{s}$. The red line is for the sharp edge of the plate exposed to the flow, while the blue line is for the blunt edge exposed to the flow. 
Figure 64: Frequency spectra for a perforated plate with a hole diameter of $6.35 \mathrm{~mm}$, an angle of incidence of 5 degrees, and a flow velocity of 5 to $30 \mathrm{~m} / \mathrm{s}$. The red line is for the sharp edge of the plate exposed to the flow, while the blue line is for the blunt edge exposed to the flow.

Figure 65: Frequency spectra for a perforated plate with a hole diameter of $6.35 \mathrm{~mm}$, an angle of incidence of 10 degrees, and a flow velocity of 5 to $30 \mathrm{~m} / \mathrm{s}$. The red line is for the sharp edge of the plate exposed to the flow, while the blue line is for the blunt edge exposed to the flow.

Figure 66: Frequency spectra for a perforated plate with a hole diameter of $6.35 \mathrm{~mm}$, an angle of incidence of 15 degrees, and a flow velocity of 5 to $30 \mathrm{~m} / \mathrm{s}$. The red line is for the sharp edge of the plate exposed to the flow, while the blue line is for the blunt edge exposed to the flow.

Figure 67: Frequency spectra for a perforated plate with a hole diameter of $6.35 \mathrm{~mm}$, an angle of incidence of 20 degrees, and a flow velocity of 5 to $30 \mathrm{~m} / \mathrm{s}$. The red line is for the sharp edge of the plate exposed to the flow, while the blue line is for the blunt edge exposed to the flow.

Figure 68: Frequency spectra for a perforated plate with a hole diameter of $6.35 \mathrm{~mm}$, an angle of incidence of 25 degrees, and a flow velocity of 5 to $30 \mathrm{~m} / \mathrm{s}$. The red line is for the sharp edge of the plate exposed to the flow, while the blue line is for the blunt edge exposed to the flow.

Figure 69: Frequency spectra for a perforated plate with a hole diameter of $6.35 \mathrm{~mm}$, an angle of incidence of 30 degrees, and a flow velocity of 5 to $30 \mathrm{~m} / \mathrm{s}$. The red line is for the sharp edge of the plate exposed to the flow, while the blue line is for the blunt edge exposed to the flow. 
Figure 70: Frequency spectra for a perforated plate with a hole diameter of $6.35 \mathrm{~mm}$, an angle of incidence of 35 degrees, and a flow velocity of 5 to $30 \mathrm{~m} / \mathrm{s}$. The red line is for the sharp edge of the plate exposed to the flow, while the blue line is for the blunt edge exposed to the flow.

Figure 71: Frequency spectra for a perforated plate with a hole diameter of $6.35 \mathrm{~mm}$, an angle of incidence of 40 degrees, and a flow velocity of 5 to $30 \mathrm{~m} / \mathrm{s}$. The red line is for the sharp edge of the plate exposed to the flow, while the blue line is for the blunt edge exposed to the flow.

Figure 72: Frequency spectra for a gap width of $3.175 \mathrm{~mm}$, an angle of incidence of 0 degrees, and a flow velocity of 5 to $30 \mathrm{~m} / \mathrm{s}$.

Figure 73: Frequency spectra for a gap width of $3.175 \mathrm{~mm}$, an angle of incidence of 5 degrees, and a flow velocity of 5 to $30 \mathrm{~m} / \mathrm{s}$.

Figure 74: Frequency spectra for a gap width of $3.175 \mathrm{~mm}$, an angle of incidence of 10 degrees, and a flow velocity of 5 to $30 \mathrm{~m} / \mathrm{s}$.

Figure 75: Frequency spectra for a gap width of $3.175 \mathrm{~mm}$, an angle of incidence of 15 degrees, and a flow velocity of 5 to $30 \mathrm{~m} / \mathrm{s}$.

Figure 76: Frequency spectra for a gap width of $3.175 \mathrm{~mm}$, an angle of incidence of 20 degrees, and a flow velocity of 5 to $30 \mathrm{~m} / \mathrm{s}$.

Figure 77: Frequency spectra for a gap width of $3.175 \mathrm{~mm}$, an angle of incidence of 25 degrees, and a flow velocity of 5 to $30 \mathrm{~m} / \mathrm{s}$.

Figure 78: Frequency spectra for a gap width of $3.175 \mathrm{~mm}$, an angle of incidence of 30 degrees, and a flow velocity of 5 to $30 \mathrm{~m} / \mathrm{s}$.

Figure 79: Frequency spectra for a gap width of $3.175 \mathrm{~mm}$, an angle of incidence of 35 degrees, and a flow velocity of 5 to $30 \mathrm{~m} / \mathrm{s}$. 
Figure 80: Frequency spectra for a gap width of $3.175 \mathrm{~mm}$, an angle of incidence of 40 degrees, and a flow velocity of 5 to $30 \mathrm{~m} / \mathrm{s}$.

Figure 81: Frequency spectra for a gap width of $6.35 \mathrm{~mm}$, an angle of incidence of 0 degrees, and a flow velocity of 5 to $30 \mathrm{~m} / \mathrm{s}$.

Figure 82: Frequency spectra for a gap width of $6.35 \mathrm{~mm}$, an angle of incidence of 5 degrees, and a flow velocity of 5 to $30 \mathrm{~m} / \mathrm{s}$.

Figure 83: Frequency spectra for a gap width of $6.35 \mathrm{~mm}$, an angle of incidence of 10 degrees, and a flow velocity of 5 to $30 \mathrm{~m} / \mathrm{s}$.

Figure 84: Frequency spectra for a gap width of $6.35 \mathrm{~mm}$, an angle of incidence of 15 degrees, and a flow velocity of 5 to $30 \mathrm{~m} / \mathrm{s}$.

Figure 85: Frequency spectra for a gap width of $6.35 \mathrm{~mm}$, an angle of incidence of 20 degrees, and a flow velocity of 5 to $30 \mathrm{~m} / \mathrm{s}$.

Figure 86: Frequency spectra for a gap width of $6.35 \mathrm{~mm}$, an angle of incidence of 25 degrees, and a flow velocity of 5 to $30 \mathrm{~m} / \mathrm{s}$.

Figure 87: Frequency spectra for a gap width of $6.35 \mathrm{~mm}$, an angle of incidence of 30 degrees, and a flow velocity of 5 to $30 \mathrm{~m} / \mathrm{s}$.

Figure 88: Frequency spectra for a gap width of $6.35 \mathrm{~mm}$, an angle of incidence of 35 degrees, and a flow velocity of 5 to $30 \mathrm{~m} / \mathrm{s}$.

Figure 89: Frequency spectra for a gap width of $6.35 \mathrm{~mm}$, an angle of incidence of 40 degrees, and a flow velocity of 5 to $30 \mathrm{~m} / \mathrm{s}$.

Figure 90: Frequency spectra for a gap width of $12.7 \mathrm{~mm}$, an angle of incidence of 0 degrees, and a flow velocity of 5 to $30 \mathrm{~m} / \mathrm{s}$. 
Figure 91: Frequency spectra for a gap width of $12.7 \mathrm{~mm}$, an angle of incidence of 5 degrees, and a flow velocity of 5 to $30 \mathrm{~m} / \mathrm{s}$.

Figure 92: Frequency spectra for a gap width of $12.7 \mathrm{~mm}$, an angle of incidence of 10 degrees, and a flow velocity of 5 to $30 \mathrm{~m} / \mathrm{s}$.

Figure 93: Frequency spectra for a gap width of $12.7 \mathrm{~mm}$, an angle of incidence of 15 degrees, and a flow velocity of 5 to $30 \mathrm{~m} / \mathrm{s}$.

Figure 94: Frequency spectra for a gap width of $12.7 \mathrm{~mm}$, an angle of incidence of 20 degrees, and a flow velocity of 5 to $30 \mathrm{~m} / \mathrm{s}$.

Figure 95: Frequency spectra for a gap width of $12.7 \mathrm{~mm}$, an angle of incidence of 25 degrees, and a flow velocity of 5 to $30 \mathrm{~m} / \mathrm{s}$.

Figure 96: Frequency spectra for a gap width of $12.7 \mathrm{~mm}$, an angle of incidence of 30 degrees, and a flow velocity of 5 to $30 \mathrm{~m} / \mathrm{s}$.

Figure 97: Frequency spectra for a gap width of $12.7 \mathrm{~mm}$, an angle of incidence of 35 degrees, and a flow velocity of 5 to $30 \mathrm{~m} / \mathrm{s}$.

Figure 98: Frequency spectra for a gap width of $12.7 \mathrm{~mm}$, an angle of incidence of 40 degrees, and a flow velocity of 5 to $30 \mathrm{~m} / \mathrm{s}$.

Figure 99: Frequency spectra for a gap width of $6.35 \mathrm{~mm}$, an angle of incidence of $15^{\circ}$, and a flow velocity of $15 \mathrm{~m} / \mathrm{s}$. Results are shown for un-taped (blue \& red) and taped (green \& pink) slats. The streamwise position is varied, from before the first gap to after the last gap. For Case \#4, spectra are only shown for the $12^{\text {th }}$ to $24^{\text {th }}$ gaps..... 136

Figure 100: Overall sound pressure level vs. streamwise position for a gap width of 6.35 $\mathrm{mm}$, an angle of incidence of $15^{\circ}$, and a flow velocity of $15 \mathrm{~m} / \mathrm{s}$. Results are shown for un-taped (blue \& red) and taped (green \& pink) slats. 
Figure 101: Frequency spectra for a gap width of $6.35 \mathrm{~mm}$, an angle of incidence of $15^{\circ}$, and a flow velocity of $20 \mathrm{~m} / \mathrm{s}$. Results are shown for un-taped (blue \& red) and taped (green \& pink) slats. The streamwise position is varied, from before the first gap to after the last gap. For Case \#4, spectra are only shown for the $3^{\text {rd }}$ to $24^{\text {th }}$ gaps.

Figure 102: Overall sound pressure level vs. streamwise position for a gap width of 6.35 $\mathrm{mm}$, an angle of incidence of $15^{\circ}$, and a flow velocity of $20 \mathrm{~m} / \mathrm{s}$. Results are shown for un-taped (blue $\&$ red) and taped (green $\&$ pink) slats.

Figure 103: Frequency spectra for a gap width of $6.35 \mathrm{~mm}$, an angle of incidence of $15^{\circ}$, and a flow velocity of $25 \mathrm{~m} / \mathrm{s}$. Results are shown for un-taped (blue \& red) and taped (green \& pink) slats. The streamwise position is varied, from before the first gap to after the last gap. For Case \#4, spectra are only shown for the $3^{\text {rd }}$ to $24^{\text {th }}$ gaps. 140

Figure 104: Overall sound pressure level vs. streamwise position for a gap width of 6.35 $\mathrm{mm}$, an angle of incidence of $15^{\circ}$, and a flow velocity of $25 \mathrm{~m} / \mathrm{s}$. Results are shown for un-taped (blue \& red) and taped (green \& pink) slats.

Figure 105: Frequency spectra for a gap width of $6.35 \mathrm{~mm}$, an angle of incidence of $15^{\circ}$, and a flow velocity of $30 \mathrm{~m} / \mathrm{s}$. Results are shown for un-taped (blue \& red) and taped (green \& pink) slats. The streamwise position is varied, from before the first gap to after the last gap.

Figure 106: Overall sound pressure level vs. streamwise position for a gap width of 6.35 $\mathrm{mm}$, an angle of incidence of $15^{\circ}$, and a flow velocity of $30 \mathrm{~m} / \mathrm{s}$. Results are shown for un-taped (blue \& red) and taped (green \& pink) slats.

Figure 107: PIV results showing the average velocity field at the 24th gap for a gap width of $6.35 \mathrm{~mm}$, an angle of incidence of 0 degrees, and a flow velocity of $15 \mathrm{~m} / \mathrm{s}$. 
Figure 108: PIV results showing the average vorticity field at the 24th gap for a gap width of $6.35 \mathrm{~mm}$, an angle of incidence of 0 degrees, and a flow velocity of $15 \mathrm{~m} / \mathrm{s}$.

Figure 109: PIV results showing the average velocity field at the 24th gap for a gap width of $6.35 \mathrm{~mm}$, an angle of incidence of 0 degrees, and a flow velocity of $20 \mathrm{~m} / \mathrm{s}$.

Figure 110: PIV results showing the average vorticity field at the 24th gap for a gap width of $6.35 \mathrm{~mm}$, an angle of incidence of 0 degrees, and a flow velocity of $20 \mathrm{~m} / \mathrm{s}$.

Figure 111: PIV results showing the average velocity field at the 24th gap for a gap width of $6.35 \mathrm{~mm}$, an angle of incidence of 0 degrees, and a flow velocity of $25 \mathrm{~m} / \mathrm{s}$.

Figure 112: PIV results showing the average vorticity field at the 24th gap for a gap width of $6.35 \mathrm{~mm}$, an angle of incidence of 0 degrees, and a flow velocity of $25 \mathrm{~m} / \mathrm{s}$.

Figure 113: PIV results showing the average velocity field at the 24th gap for a gap width of $6.35 \mathrm{~mm}$, an angle of incidence of 0 degrees, and a flow velocity of $30 \mathrm{~m} / \mathrm{s}$.

Figure 114: PIV results showing the average vorticity field at the 24th gap for a gap width of $6.35 \mathrm{~mm}$, an angle of incidence of 0 degrees, and a flow velocity of $30 \mathrm{~m} / \mathrm{s}$.

Figure 115: PIV results showing the average velocity field at the 24th gap for a gap width of $6.35 \mathrm{~mm}$, an angle of incidence of 35 degrees, and a flow velocity of $15 \mathrm{~m} / \mathrm{s}$. 
Figure 116: PIV results showing the average vorticity field at the 24th gap for a gap width of $6.35 \mathrm{~mm}$, an angle of incidence of 35 degrees, and a flow velocity of $15 \mathrm{~m} / \mathrm{s}$.

Figure 117: PIV results showing the average velocity field at the 24th gap for a gap width of $6.35 \mathrm{~mm}$, an angle of incidence of 35 degrees, and a flow velocity of $20 \mathrm{~m} / \mathrm{s}$.

Figure 118: PIV results showing the average vorticity field at the 24th gap for a gap width of $6.35 \mathrm{~mm}$, an angle of incidence of 35 degrees, and a flow velocity of $20 \mathrm{~m} / \mathrm{s}$.

Figure 119: PIV results showing the average velocity field at the 24th gap for a gap width of $6.35 \mathrm{~mm}$, an angle of incidence of 35 degrees, and a flow velocity of $25 \mathrm{~m} / \mathrm{s}$. 150

Figure 120: PIV results showing the average vorticity field at the 24th gap for a gap width of $6.35 \mathrm{~mm}$, an angle of incidence of 35 degrees, and a flow velocity of $25 \mathrm{~m} / \mathrm{s}$. .150

Figure 121: PIV results showing the average velocity field at the 24th gap for a gap width of $6.35 \mathrm{~mm}$, an angle of incidence of 35 degrees, and a flow velocity of $30 \mathrm{~m} / \mathrm{s}$.

Figure 122: PIV results showing the average vorticity field at the 24th gap for a gap width of $6.35 \mathrm{~mm}$, an angle of incidence of 35 degrees, and a flow velocity of $30 \mathrm{~m} / \mathrm{s}$. 


\section{LIST OF SYMBOLS}

$D \quad$ Hole diameter

$D / t \quad$ Hole diameter over thickness ratio

d Characteristic dimension for calculating $S t$

$d_{e} \quad$ Effective diameter for calculating $S t$ for rectangular holes

$f \quad$ Frequency

$H \quad$ Gap width

$H / t \quad$ Gap width over thickness ratio

$h \quad$ Height of the wind tunnel exit section

$P \quad$ Sound pressure

$P^{*} \quad$ Normalized sound pressure

$\operatorname{Re}_{D} \quad$ Reynolds number based on gap width

$S \quad$ Width of the solid between holes, or the width of the slats

$S / t \quad$ Solid width or slat width over thickness ratio

St Strouhal number

$t \quad$ Thickness of the perforated plate or slats

$V \quad$ Velocity magnitude

$V^{*} \quad$ Dimensionless velocity magnitude

$V_{\text {mean }}$ Mean flow velocity from the wind tunnel

$w \quad$ Width of the wind tunnel exit section

$X^{*} \quad$ Dimensionless $X$-position

$Y^{*} \quad$ Dimensionless $Y$-position 
$\rho \quad$ Density of air

$\Theta \quad$ Momentum thickness

$\theta \quad$ Angle of incidence

$\omega \quad$ Vorticity

$\omega^{*} \quad$ Dimensionless vorticity 


\section{CHAPTER 1 INTRODUCTION}

\subsection{Problem}

Perforated plates are thin metal plates with a repetitive pattern of holes. These holes are most commonly circular, but may also be of different geometries. These plates are often used as a decorative feature on the exterior faces of buildings. They may be used on balconies, stairways, and windows, and sometimes as a cladding over the entire external surface of the building. An example of perforated plates used on a balcony is shown in Figure 1. These plates are selected by architects for their visual appeal and optical properties. They are typically not structural and in most cases, they could be replaced with another material such as bannisters or glass panes.

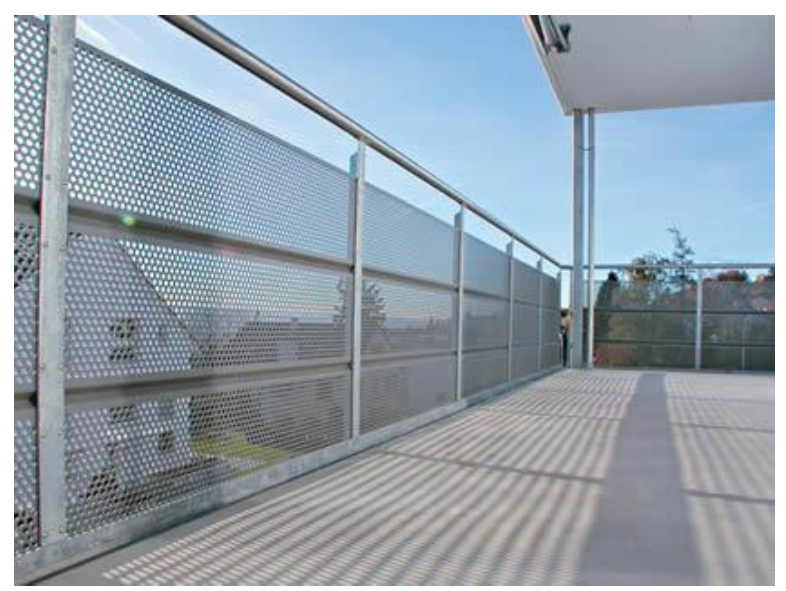

Figure 1: Perforated metal plates used on the balcony of a building (Schafer, 2017).

One major problem with perforated plates which often goes overlooked in building design is their tendency to produce noise when exposed to high winds. While this noise is 
generally broadband, for certain perforation geometries, wind velocities, and wind directions, strong tonal noise is produced. The level of this tonal noise can be much higher than that of the broadband noise. In addition, even at the same noise level, tonal noise is perceived as more annoying than broadband noise. ISO standard 1996:1 defines tonal noise as "sound characterized by a single frequency component or narrow-band components that emerge audibly from the total sound" and specifies an adjustment of 3 to 6 decibels for tonal noise sources (ISO, 2003). This means that, for the purpose of meeting noise regulations, 3 to $6 \mathrm{~dB}$ is added to the measured overall sound pressure level of a tonal noise source as a penalty for the perceived annoyance level. This is a significant increase, as a 6 $\mathrm{dB}$ increase corresponds to a doubling of the sound pressure in Pascals.

In architectural applications, the noise produced by perforated plates is an irritant to inhabitants of the building and the surrounding area. In some extreme cases, these problems attract media attention, which creates negative publicity for the designers and architects of the building. This often results in costly retrofitting in attempt to eliminate the problem. In most cases, the only known solution is to remove all the plates from the building and replace them with a different product. In order to prevent these problems from occurring in the future and to repair existing problems in a timely and cost-effective manner, a thorough understanding of the mechanism which generates this noise is required.

In this thesis, a two-dimensional model of a perforated plate is used to study the flow over a perforated plate for various geometries at various angles of incidence and flow velocities. This work consists of two main parts. First, the acoustic response of the plates is measured using a microphone positioned downstream of the plate. Secondly, phase- 
locked particle image velocimetry (PIV) is used to study the details of the flow field and the mechanism of tone generation. The objective of this research is to study the flow over perforated plates and identify the conditions which are favorable to the production of tonal noise. This will aid in designing against this noise in the future.

\subsection{Thesis Outline}

This thesis consists of five chapters. After this Introduction, Chapter 2: Literature Review gives an overview of the existing literature relevant to the following research. This includes general cases of shear layer instability and aeroacoustic feedback mechanisms and more specific work related to flow over perforated plates. Chapter 3: Experimental Apparatus gives a description of the experimental test facility and the acoustic and particle image velocimetry measurements used in the research. Chapter 4: Results and Discussion provides complete results of the acoustic and PIV experiments, as well as a discussion of the findings. Finally, in Chapter 5: Conclusions, the results are summarized and recommendations for future work are provided. 


\section{CHAPTER 2 LITERATURE REVIEW}

\subsection{Introduction}

In this chapter, flow instability is reviewed as it relates to free shear flows. The effect of an unstable shear flows impinging on a solid surface is discussed. This produces periodic excitation due to a feedback mechanism. In the absence of resonance or elastic effects, this is known as the fluid dynamic feedback mechanism. After describing the fundamental concepts of the fluid dynamic mechanism, the theory is applied to the specific case of flow over perforated plates. This case is characteristic of the fluid dynamic mechanism, with tonal noise produced for a specific range of flow velocities and angles of incidence to the plate. The frequency of these tones increases with increasing flow velocity and decreases with increasing impingement distance. Finally, the simplification of a perforated plate into a two-dimensional geometry, which is the approach taken in this research, is discussed.

\subsection{Shear Layer Instability}

A fluid flow is defined as unstable if an infinitesimally small disturbance becomes amplified to have a noticeable effect on the flow field. In unstable flows, these disturbances grow into vortex-like structures which produce velocity and pressure fluctuations and noise. As early as 1880 , Rayleigh showed that two-dimensional flow which contains a 
change in curvature, or an inflection point, may be unstable (Rayleigh, 1880). This was done mathematically, by applying a disturbance to the equations of motion of a twodimensional flow field. Tollmien expanded on this in 1935 to prove that the presence of this inflection point is a sufficient condition for instability in certain two-dimensional inviscid laminar flows (Tollmien, 1935).

A common example of an unstable fluid flow is a free shear layer flow. A free shear layer is formed when two parallel fluid flows with different velocities merge together. The boundary layers of the two flows come together to form a region of high vorticity. The velocity of one of the flows can be zero. For example, in flow over the leading edge of a cavity, the moving fluid merges with the nearly stagnant fluid inside the cavity, creating a free shear layer across the cavity surface. The high velocity flow rotates towards the low velocity flow, producing high vorticity. The shear layer is called "free" because it is not bounded by a solid surface. The velocity profile of a free shear layer is characterized by an inflection point near the plane where the two fluid streams meet. Therefore, these flows are inherently unstable, leading to the production of vortices and turbulence. Common free shear flows include jets, wakes, and mixing layers.

Michalke used Rayleigh's stability theory to numerically investigate the instability characteristics for a two-dimensional velocity profile of the form in equation (1) (Michalke, 1964; Michalke, 1965; Michalke, 1972). This velocity profile is typical of a free shear layer with an inflection point.

$$
U(y)=0.5(1+\tanh y)
$$


Michalke calculated the theoretical growth rate of a small disturbance in this velocity field using both temporal and spatial amplification. If small disturbances grow with time or space, the flow field is said to be unstable. The growth rate of disturbances was found to be a function of the frequency of the disturbance. For low frequencies, this flow field is always unstable, reaching a maximum instability at a specific frequency, near the middle of the unstable range.

The frequency of the disturbance is expressed as the Strouhal number based on momentum thickness. The Strouhal number is a dimensionless number for the frequency in oscillating flows. It is given by equation (2) below, where $f$ is the frequency of oscillations, $d$ is the characteristic dimension, and $V_{\text {mean }}$ is the mean velocity of the flow. The characteristic dimension is selected based on the geometry of the problem. In this case, $d$ is defined as the momentum thickness, $\Theta$.

$$
S t=\frac{f * d}{V_{\text {mean }}}
$$

Freymuth conducted experiments on the instability of the free shear layer of both a round and a planar jet (Freymuth, 1966). A disturbance with a defined frequency was produced by a loudspeaker at the outlet of the jet. The amplitude of the excitation and the Reynold's number of the jets were adjusted. Hot wire measurements were taken at various downstream points to calculate the growth of these disturbances. It was found that the amplitude of the excitation increased the size of the initial disturbance, but had no effect on the growth rate of the disturbance or the maximum amplitude of the disturbance. This is shown in Figure 2 for the round jet with a diameter of $7.5 \mathrm{~cm}$ and an exit velocity of $8 \mathrm{~m} / \mathrm{s}$. 
As the amplitude of excitation was increased, the amplitude of the disturbance relative to the mean flow velocity increased, moving the starting point of the curves higher on the $y$ axis. The disturbances then grew exponentially, which is given by linear lines on a logarithmic scale. The exponential growth rate of the disturbance is given by the slope of the lines. For the case of increasing amplitude of excitation, the slopes of all the lines are the same, so the disturbances grew at the same rate.

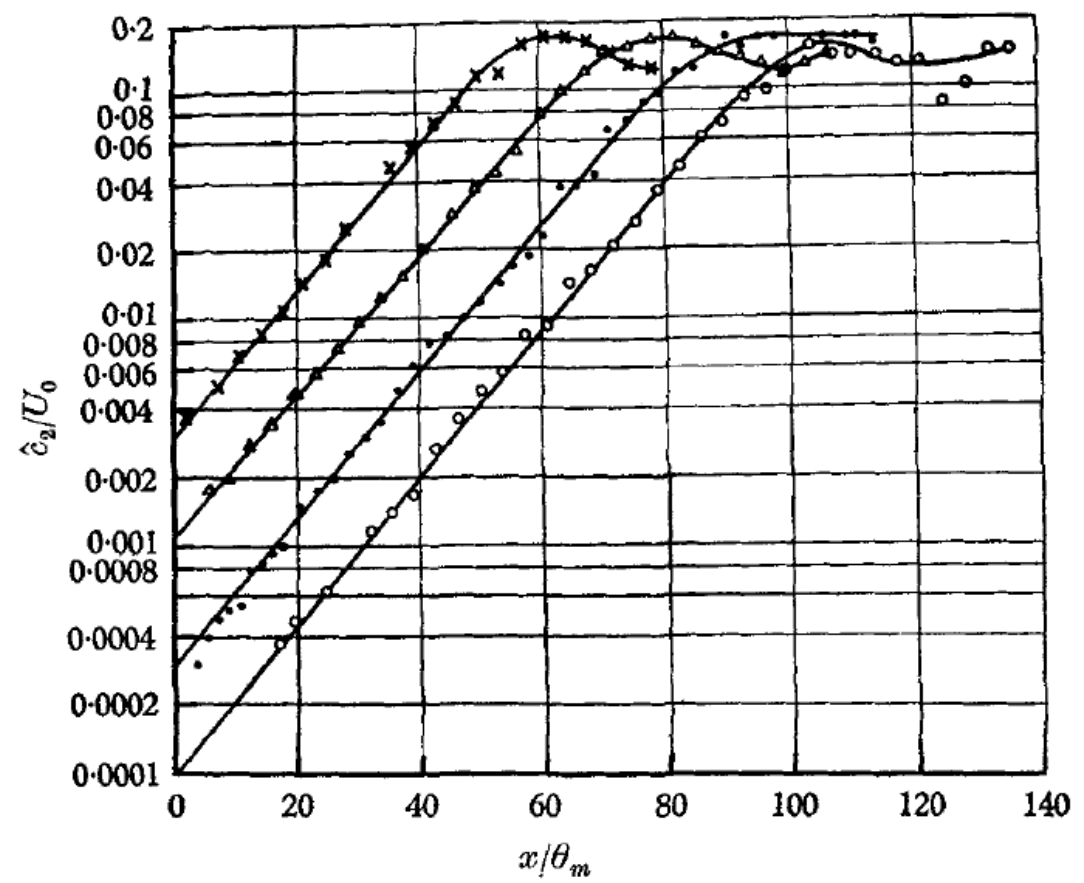

Figure 2: Dependence of the growth rate of disturbances on the amplitude of excitation for a circular jet with $D=7.5 \mathrm{~cm}, U_{0}=8 \mathrm{~m} / \mathrm{s}, R e_{\Theta}=122: \circ, 70 \mathrm{~dB}$; $\bullet, 80 \mathrm{~dB} ; \Delta, 90 \mathrm{~dB} ; \mathrm{x}, 100 \mathrm{~dB}$ (Freymuth, 1966).

The frequency of excitation was found by Freymuth to have a large effect on the growth rate of disturbances (Freymuth, 1966). The results are shown in Figure 3 for the round jet with a diameter of $7.5 \mathrm{~cm}$, an exit velocity of $16 \mathrm{~m} / \mathrm{s}$, and a variable Strouhal 
number of excitation. As $S t_{\Theta}$ was increased, the slope of the lines increased, and the disturbances grew at a faster rate. Similar results were produced for the planar jet.

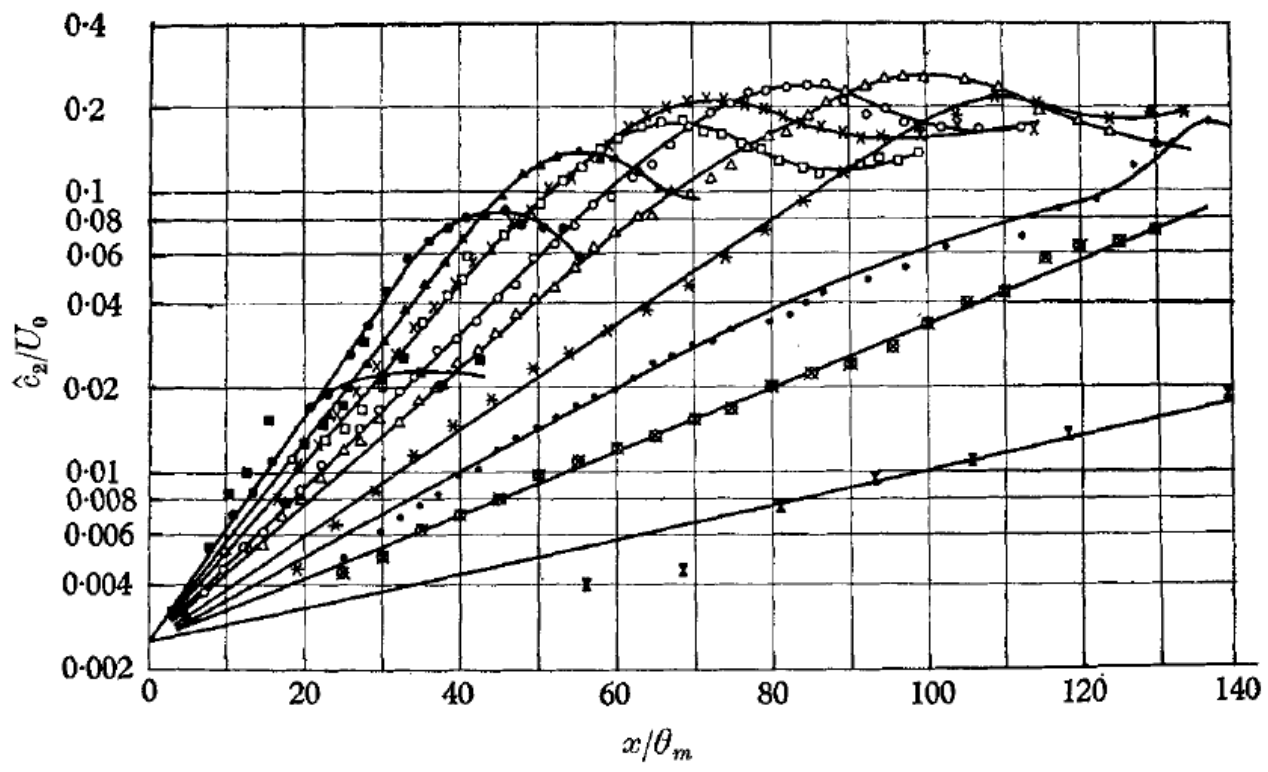

Figure 3: Dependence of the growth rate of disturbances on the Strouhal number for a circular jet with $D=7.5 \mathrm{~cm}, U_{0}=16 \mathrm{~m} / \mathrm{s}: X, S t_{\Theta}=0.0020 ; \otimes, S t_{\Theta}=0.0040$; $\bullet, S t_{\Theta}=0.0050 ; *, S t_{\Theta}=0.0070 ; \Delta, S t_{\Theta}=0.0080 ; \circ, S t_{\Theta}=0.0090 ; x, S t_{\Theta}=0.0100 ;$ $\square, S t_{\Theta}=0.0118 ; \Delta, S t_{\Theta}=0.0148 ; \bullet, S t_{\Theta}=0.0176 ; \square, S t_{\Theta}=0.0234$ (Freymuth, 1966).

In Figure 4, the experimental results of Freymuth are compared to the numerical results of Michalke using both the theory of spatial amplification and temporal amplification (Freymuth, 1966; Michalke, 1965). The experimental results for both the round jet and the planar jet agreed closely with the numerical results. For low frequency, the data agreed better with the spatial amplification case, while at higher frequencies, the agreement was better with the temporal amplification case. The experimental results end at $S t_{\Theta}=0.0234$, since the jet could not be excited at higher frequencies than this. The peak of these curves is the Strouhal number at which disturbance grew at the fastest rate and the free shear layer was most unstable. For both the numerical and experimental results, this 
occurred at a Strouhal number of around 0.0176. The shear layer was most unstable for the range of $S t_{\Theta}=0.01$ to 0.025 .

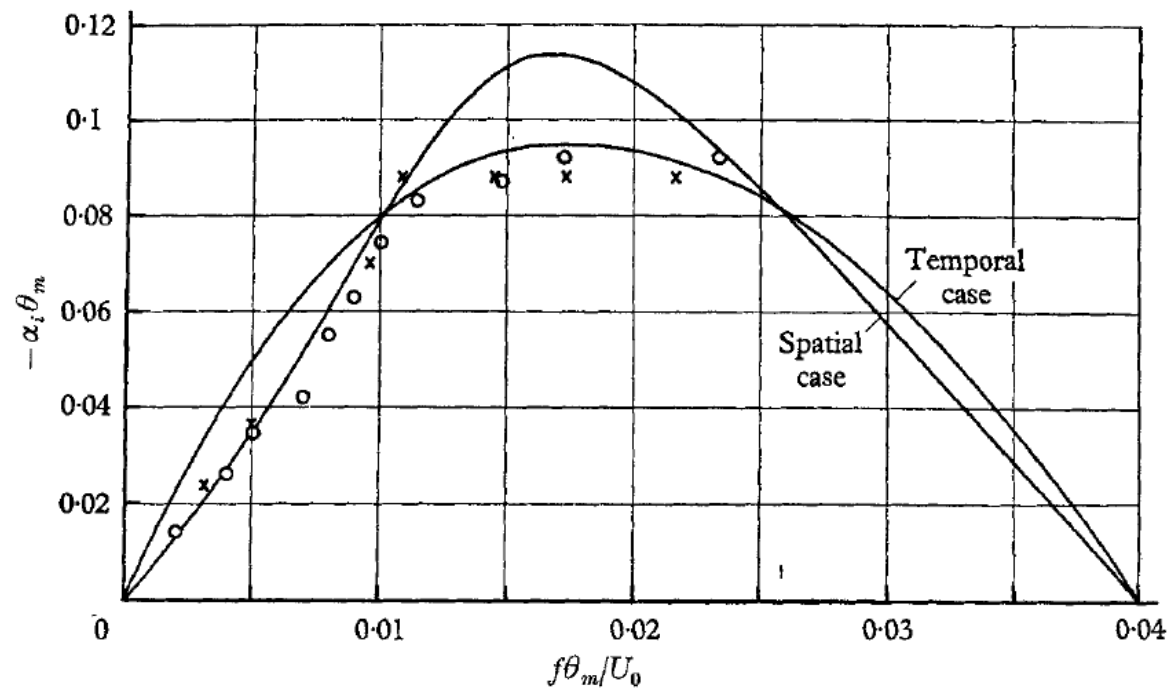

Figure 4: Growth rate of disturbances as a function of Strouhal number based on momentum thickness, $S t_{\Theta}$. The lines are the numerical results of Michalke for both spatial and temporal amplification of disturbances; and the symbols are the experimental results of Freymuth: O, round jet; X, planar jet (Michalke, 1965; Freymuth, 1966).

\subsection{Impinging Shear Flows}

There are three general classes of feedback mechanisms leading to flow-induced oscillation (Naudascher, 1967). These are the fluid-resonant mechanism, the fluid-elastic mechanism, and the fluid-dynamic mechanism. In some cases, more than one of these mechanisms can couple together in the same system.

In the fluid-resonant mechanism, instabilities in the flow excite resonant effects in a confined geometry. Examples of this mechanism are standing waves in pipes and closed cavities. The frequency of these oscillations is dictated by the geometry of the resonator and its resonance frequency. 
In the fluid-elastic mechanism, oscillations are caused by elastic movement or vibration of a solid boundary. An example of a fluid-elastic instability is vibrating tube bundles in a heat exchanger which couple with the flow under certain operating conditions. The frequency of oscillations is dictated by the natural frequency of the vibrating component.

Finally, the fluid-dynamic mechanism is caused by inherent instabilities in the flow, independent of resonance or elastic effects. Fluid-dynamic feedback is caused when an unstable flow impinges on a solid boundary. This leads to a pressure fluctuation which is felt upstream at the initial region of the flow instability. This closes a feedback loop and leads to enhancement of the instability at its source. The fluid-dynamic mechanism is illustrated in Figure 5. Here, a free shear layer and flow instability originate at the separation edge. Vortices form in the flow and impinge on the downstream corner. The feedback from this impingement enhances the oscillation, leading to the periodic shedding and impingement of vortices. The frequency of oscillations is defined by the free stream velocity, $U$, and the impingement length, $L$. As shown in Figure 5(a), the frequency increases linearly with increasing flow velocity within a certain range of instability. The frequency decreases linearly with increasing impingement length, as shown in Figure 5(b). The frequency of oscillations may have multiple modes or stages, with a frequency jump between each stage. 


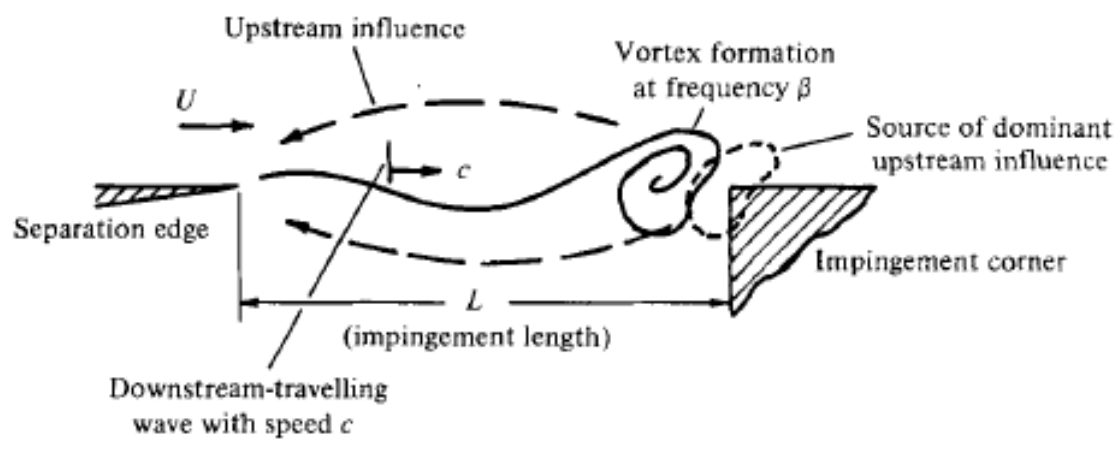

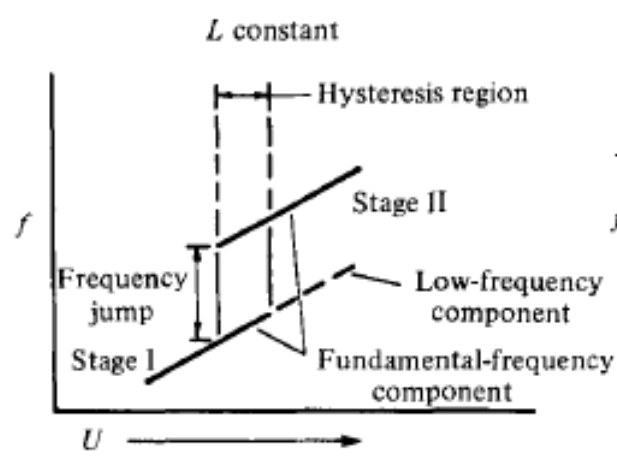

(a)

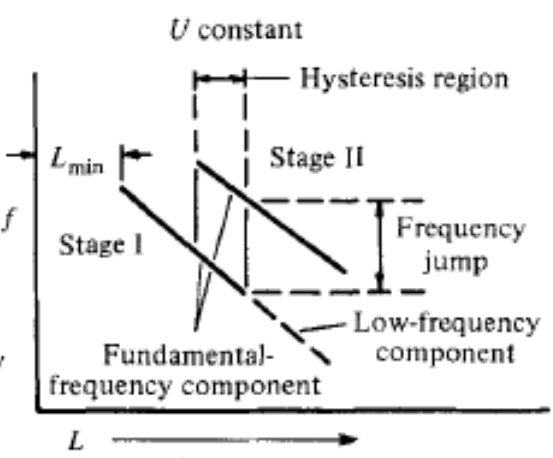

(b)

Figure 5: Fluid-dynamic mechanism caused by flow impingement on an edge; and the frequency variation with (a) varying free-stream velocity $U$ and constant impingement length $L$, and (b) varying impingement length $L$ and constant freestream velocity $U$ (Knisely \& Rockwell, 1982).

Examples of flow configurations leading to oscillation generated by the fluiddynamic mechanism are shown in Figure 6. In all of these cases, an unstable free shear layer originates at a jet exit or a mixing layer. This leads to the formation of vortical structures, which impinge on a downstream surface, which may be a plate, cylinder, edge, or others shown in Figure 6. This impingement sends a pressure wave which is felt upstream at the source of the free shear layer, which enhances the instability. The velocity of the flow and the dimensions of the solid boundaries may lead to periodic shedding and impingement of vortices at a defined frequency. 


\begin{tabular}{|l|l|c|}
\hline & PLANAR & PXISYMMETRIC \\
JETS
\end{tabular}

Figure 6: Basic configurations of shear-layer and impingement-edge geometries that produce self-sustained oscillations (Rockwell \& Naudascher, 1979).

Flow over perforated plates may produce oscillations due to the fluid-dynamic mechanism. This geometry is similar to several of the cases shown in Figure 6, particularly the "jet-surface," "jet-plate," and "mixing layer-edge." However, it has some of its own unique characteristics, which will be discussed in the following section and in Chapter 4: Results and Discussion.

\subsection{Flow Over Perforated Plates}

Extensive work has been done in the literature to study flow over perforated plates and orifices at parallel and perpendicular angles of incidence (Howe, 1981; Howe, 1981; Nelson, 1982; Rockwell \& Naudascher, 1979; Tsui \& Flandro, 1977). The applications of 
these studies include duct flow and perforated jet engine liners. For these applications, the flow is typically in a parallel or perpendicular direction.

Flow through a perforated plate or orifice at a perpendicular direction may produce tonal noise due to a separation of the incident flow at the upstream corner of the orifice and an impingement of this flow near the downstream corner. This is illustrated in Figure 7(a). This effect occurs only for sharp-edged holes with a diameter of 1 to 2 times their length $(D / t=1-2)($ Heller \& Franken, 1988). For holes with a $D / t$ less than 1 , reattachment occurs before the downstream corner of the orifice. For holes with a $D / t$ greater than 2, reattachment does not occur within the length of the orifice. In both of these cases, tonal noise is not produced.

Similarly, parallel angles of incidence, or grazing flow, has been studied by many researchers for a variety of geometries, including perforated plates, orifices, and cavities. The case of tonal noise generation by grazing flow over a perforated surface was investigated by Celik and Rockwell in 2002, with further details found in the works cited therein (Celik \& Rockwell, 2002). In general, these tones are generated by the periodic impingement of vortices on the downstream corner of the holes, as shown in Figure 7(b). This periodic impingement causes a feedback which is felt upstream, leading to the repeated initiation of vortices at the upstream edge. This mechanism may be coupled with resonant or elastic effects, but can also occur purely due to the fluid-dynamic mechanism. 
a)

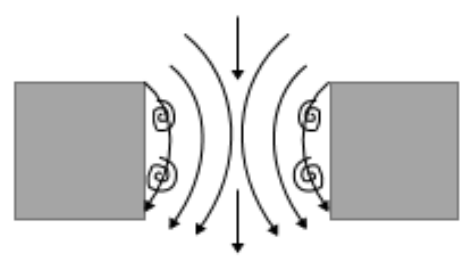

b)

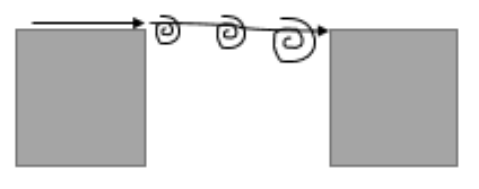

c)

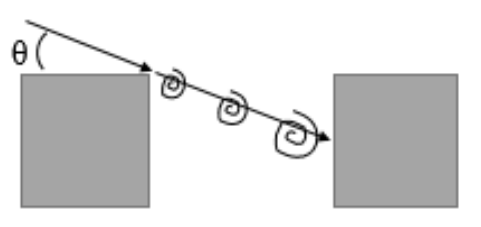

Figure 7: Flow over an orifice with (a) perpendicular angle of incidence, (b) parallel angle of incidence, (c) oblique angle of incidence.

For architectural applications, a defining feature of the flow over perforated plates is its varying angle of incidence. This results in the case of an oblique angle of incidence, shown in Figure 7(c), which is clearly distinct from the cases of perpendicular or parallel flow. With these architectural applications receiving increased attention, several experimental studies have recently been done to investigate the effect of the angle of incidence on the noise produced by perforated plates. Feng measured the acoustic response of five different geometries of perforated plates at an angle of incidence of $0^{\circ}$ to $90^{\circ}$ and a flow velocity of 3 to $15 \mathrm{~m} / \mathrm{s}$ (Feng, 2012). These five plates had a thickness, $t$, of $1.5 \mathrm{~mm}$ (1/16 inch) and a hole diameter, $D$, of $8 \mathrm{~mm}, 10 \mathrm{~mm}, 11 \mathrm{~mm}, 12 \mathrm{~mm}$, and $20 \mathrm{~mm}$. This corresponds to a $D / t$ of 5.3 to 13.3 . The solid width over thickness ratio, $S / t$, ranged from 6.67 to 20 . These geometric parameters are defined in Figure 8, and the values tested by Feng are summarized in Table 1.

Table 1: Dimensions for the perforated plates tested by Feng (2012).

\begin{tabular}{|l|l|l|l|l|}
\hline Plate \# & $\mathbf{t}(\mathbf{m m})$ & $\mathbf{D}(\mathbf{m m})$ & $\mathbf{D} / \mathbf{t}$ & $\mathbf{S} / \mathbf{t}$ \\
\hline 3 & 1.5 & 8 & 5.3 & 6.7 \\
\hline 5 & 1.5 & 10 & 6.7 & 7.3 \\
\hline 4 & 1.5 & 11 & 7.3 & 8 \\
\hline 2 & 1.5 & 12 & 8 & 10 \\
\hline 1 & 1.5 & 20 & 13.3 & 20 \\
\hline
\end{tabular}




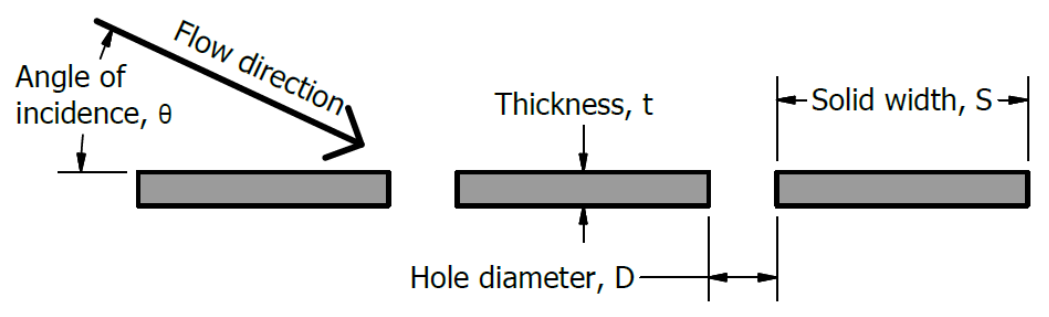

Figure 8: Geometric parameters of interest for a perforated plate.

Tonal peaks were observed for these plates for flow at angles of incidence between 10 and 30 degrees, where 0 degrees represents the case of grazing flow, or flow parallel to the plate. The strongest tones were found to occur at $\theta=15$ degrees. Beyond this range of angles, the noise was found to be broadband.

Figure 9 shows the acoustic results of Feng for an angle of incidence of 15 degrees and a velocity of $15 \mathrm{~m} / \mathrm{s}$. These frequency spectra are presented in the $\mathrm{dB}$ scale with $1 / 3$ octave bands. Results are shown for each of the five different plate geometries and a flow velocity of $15 \mathrm{~m} / \mathrm{s}$. At this velocity, a tonal peak was observed in the 2000 to $2500 \mathrm{~Hz}$ frequency bands. The peak for the plate with an $8 \mathrm{~mm}$ hole diameter is the largest. When the flow velocity was reduced to $12 \mathrm{~m} / \mathrm{s}$, the peak for the plate with an $8 \mathrm{~mm}$ hole diameter decreased, while the peaks for the plates with a hole diameter of $10 \mathrm{~mm}, 11 \mathrm{~mm}$, and $12 \mathrm{~mm}$ increased. The frequency of these peaks decreased to the $1000 \mathrm{~Hz}$ frequency band. Therefore, there appeared to be an ideal flow velocity to produce tones for a given plate geometry. This ideal velocity was greater for plates with smaller hole diameters. The frequency of the tonal peaks increased with increasing flow velocity. 
Figure 10 shows the frequency spectrum for the plate with an $8 \mathrm{~mm}$ hole diameter for a variable flow velocity. The frequency of the peak increased linearly with increasing flow velocity. This is characteristic of the fluid dynamic mechanism, as shown in Figure 5.

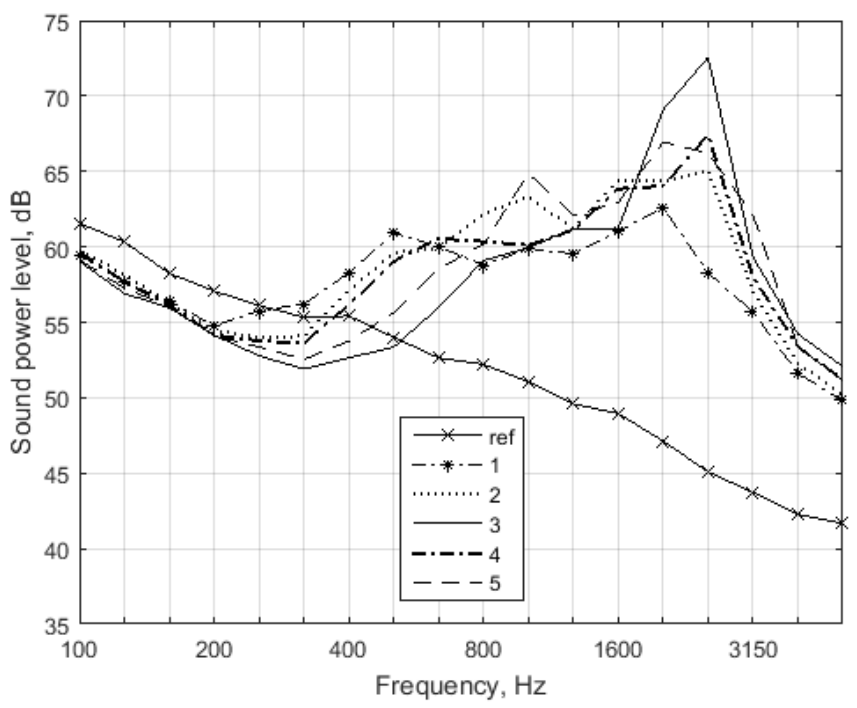

Figure 9: Results of Feng for an angle of incidence of $15^{\circ}$ and a velocity of $15 \mathrm{~m} / \mathrm{s}$ for a hole diameter of $20 \mathrm{~mm}$ (plate 1), $12 \mathrm{~mm}$ (plate 2), $11 \mathrm{~mm}$ (plate 4), $10 \mathrm{~mm}$ (plate 5), and $8 \mathrm{~mm}$ (plate 3) (Feng, 2012).

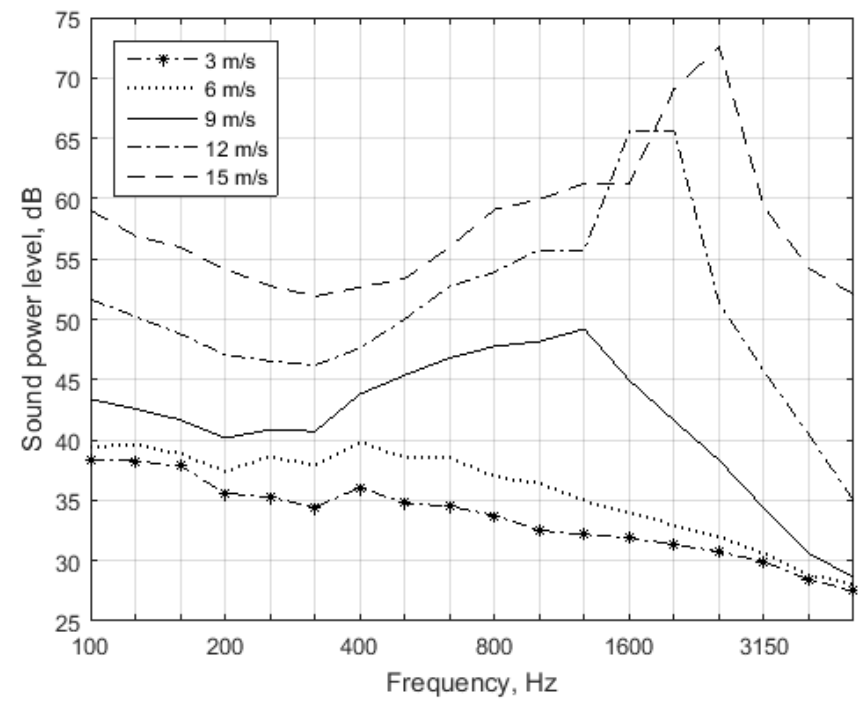

Figure 10: Results of Feng for an angle of incidence of $15^{\circ}$, a hole diameter of $8 \mathrm{~mm}$ (plate 3) and variable velocity (Feng, 2012). 
In a similar study, Blinet et al. (2015) identified tonal peaks for perforated plates with a $D / t$ of 4 to 8 . The angle of incidence of the flow at which these tones were identified ranged from 5 to 20 degrees. These authors found that the direction that the plate was oriented in the wind can have a large influence in the produced tones. The perforated plates that they tested were manufactured by punching the holes into sheet metal, which is the common manufacturing technique for perforated plates. The resulting holes have a blunt edge on the side where the punch enters the plate and a sharp edge on the side where the punch exits the plate. By orienting the plate with the sharp edge or the blunt edge exposed to the flow, different results were obtained. In Figure 11, a plate with a hole diameter of 20 $m m$ was tested at an angle of incidence of 10 degrees and a wind velocity of $15 \mathrm{~m} / \mathrm{s}$. With the blunt edge of the plate exposed to the flow, a large peak was measured at a frequency of $2000 \mathrm{~Hz}$. With the sharp edge exposed to the flow, this peak was not observed.
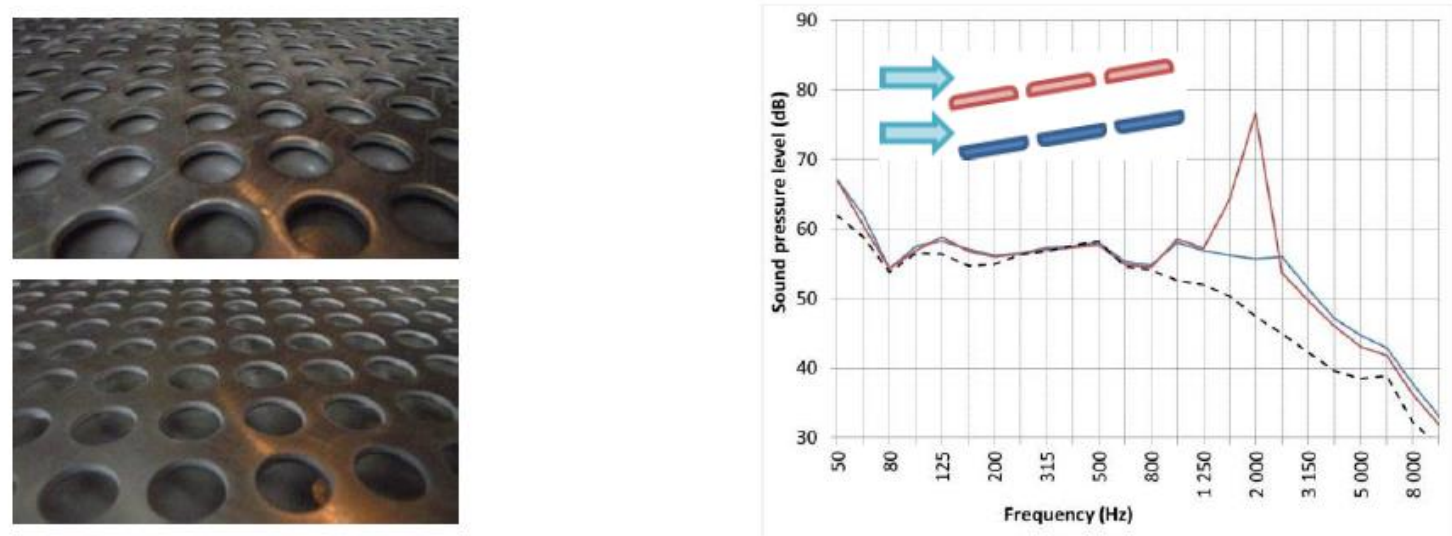

Figure 11: Acoustic results of Blinet et al. (2015) for a hole diameter of $20 \mathrm{~mm}$, an angle of incidence of $10^{\circ}$ and a velocity of $15 \mathrm{~m} / \mathrm{s}$. The red line is the result with the blunt edge of the plate exposed to the flow, and the blue line is the result with the sharp edge of the plate exposed to the flow. 
The few existing studies on flow over perforated plates highlight the strong effect of the main parameters on the produced tonal noise. The mechanism appears to be typical of the fluid dynamic mechanism. The frequency of oscillations increases with increasing flow velocity and decreases with increasing impingement distance, or hole diameter. However, none of the existing literature has studied the flow field around the plates or looked at the details of the sound generation mechanism which produces these tones. These unresolved issues are the focus of this thesis.

\subsection{Simplification from Circular to Rectangular Cavities}

In this thesis, the circular holes of a perforated plate are simplified by long rectangular gaps. This is done to simplify the problem to be predominantly two dimensional. This makes the physics easier to study and makes particle image velocimetry flow visualization much more feasible and reliable. Once the two-dimensional case is well understood, this work can be extended to three-dimensional circular holes.

Similar simplifications from circular to rectangular geometries have been done in the literature. Bruggeman proposed equation (3) below for calculating the effective diameter for rectangular cavities, where $d_{e}$ is the effective diameter and $H$ is the length of the cavity in the flow direction (Bruggeman, 1987). This effective diameter is used as the characteristic dimension for calculating the Strouhal number for rectangular cavities. This produces a Strouhal number equivalent to that of a circular cavity with a diameter, $D=H$. Therefore, a rectangular cavity with a length of $H$ behaves similarly to a circular cavity with a diameter which is $(4 / \pi)$ times larger than $H$. 


$$
d_{e}=\left(\frac{4}{\pi}\right) H
$$

Ziada and Shine used the relationship developed Bruggeman for calculating the Strouhal number for flow over side branches in a pipe (Ziada \& Shine, 1999). For side branches with square or rectangular cross-sections, the Strouhal number calculated using the effective diameter was said to be equivalent to the Strouhal number for a circular side branch using the actual diameter.

In this research, equation (3) is used when calculating the Strouhal number for the rectangular gaps. This produces a Strouhal number which is comparable to that of circular holes. 


\section{CHAPTER 3 EXPERIMENTAL APPARATUS}

\subsection{Test Facility}

In order to experimentally study the flow over perforated plates, a custom test facility was designed, manufactured, and assembled. The test section features a frame mounted on a hinge at the exit of an open-loop wind tunnel. The test plate is mounted to this frame and pivoted about the hinge to different angle of incidence. The facility allows both acoustic and particle image velocimetry measurements to be taken. In the following section, the test facility is described, starting with the wind tunnel, followed by the test section itself. Then, the acoustic and PIV measurement techniques are described, followed by a discussion of some geometric inconsistencies inherent to the experimental apparatus.

\subsubsection{Wind tunnel}

An open-loop wind tunnel is used for all the experiments. The air flow for this wind tunnel is supplied by a Sheldons Mill Exhauster 10-blade centrifugal blower. This blower is powered by a Toshiba EQP III 3-phase induction motor with a Toshiba Tosvert-130 G2+ transistor inverter. The velocity of the wind tunnel is controlled by changing the frequency of the motor. The relationship between the motor frequency and the exit velocity is calculated using a pitot tube located in the center of the wind tunnel exit section and a Fluke 922 airflow meter. This pitot tube is removed during testing to avoid an obstruction of the flow upstream of the test section. The relationship between the motor frequency and the 
exit velocity is found to be $0.7167(\mathrm{~m} / \mathrm{s}) / \mathrm{Hz}$. For a maximum velocity of $30 \mathrm{~m} / \mathrm{s}$, this corresponds to a maximum blower frequency of $41.9 \mathrm{~Hz}$. Because the blower has 10 blades, this leads to a blade passing frequency of $419 \mathrm{~Hz}$. This frequency is much lower than that of the flow-generated tonal noise produced in the experiments, which is in the range of 1 to $5 \mathrm{kHz}$. The blade passing frequency is also at a single frequency, while the tonal noise produced by the plates has a broader frequency peak. Therefore, the blade passing frequency can be easily distinguished from the tonal noise and can be filtered out or ignored. The inverter also emits noise at the chopping frequency. This frequency is adjustable, but is set to $1500 \mathrm{~Hz}$ during the acoustic experiments. Like the blade passing frequency, the chopping frequency is at a single frequency, and can be easily distinguished from the broad peaks produced by the plates.

Downstream from the blower, the wind tunnel features an expansion section, followed by a settling chamber, a contraction section, and a rectangular exit section. The settling chamber includes a series of screens to reduce the unsteadiness and swirl in the flow, while the contraction section is designed to produce uniform flow at the wind tunnel exit. The rectangular exit section is $711 \mathrm{~mm}$ ( 28 inches) wide by $216 \mathrm{~mm}$ ( 8.5 inches) high by $502 \mathrm{~mm}$ (19.75 inches) long and is used to direct the flow towards the test section and for mounting of the test section.

The flow velocity of the wind tunnel is varied in $5 \mathrm{~m} / \mathrm{s}$ increments from 0 to $30 \mathrm{~m} / \mathrm{s}$. This corresponds to a Reynolds number based on gap width, $\operatorname{Re}_{D}$, of up to $2.5 \times 10^{4}$, for a gap width of $12.7 \mathrm{~mm}$. 


\subsubsection{Test section}

A frame is mounted to the exit section of the wind tunnel and is used to firmly support the test plates at the desired angle. In Figure 12, this frame is shown fixed to the wind tunnel exit section. Figure 13 shows a horizontal cross-sectional view of the same. The frame is made from $25.4 \mathrm{~mm}$ ( 1 inch) by $50.8 \mathrm{~mm}$ ( 2 inch) by $3.175 \mathrm{~mm}(1 / 8 \mathrm{inch})$ thick Aluminum angle brackets. The frame has three sides, with a top and bottom bracket and a vertical bracket. The vertical bracket is on the upstream side of the test section and has two hinges mounted to it, one at the top and one at the bottom. The downstream side of the frame is left open with no bracket, to avoid adding any restrictions or additional noise sources in the flow. The part of the frame which is exposed to the flow is $431.8 \mathrm{~mm}$ (17 inches) high (Z-direction in Figure 13) by $914.4 \mathrm{~mm}$ (36 inches) long ( $X$-direction in Figure 13). The height of the frame is twice that of the height of the wind tunnel exit section, which minimizes the effect of flow around the top and bottom edges of the frame. The length is sufficiently long to allow development of the flow.

The top and bottom brackets extend past the vertical bracket and are bolted to two support brackets on the side of the wind tunnel exit section. The top and bottom support brackets are made from $50.8 \mathrm{~mm}$ ( 2 inch $)$ by $50.8 \mathrm{~mm}(2 \mathrm{inch})$ by $3.175 \mathrm{~mm}(1 / 8 \mathrm{inch})$ thick Aluminum angle bracket. They have slots machined into them which allow the top and bottom supports to slide along their length, changing the angle of incidence of the frame relative to the wind tunnel flow. These support brackets are fixed to the wind tunnel exit section with a threaded rod which is $12.7 \mathrm{~mm}(1 / 2 \mathrm{inch})$ in diameter. The threaded rod goes 
through a flange at the top and bottom of the exit section. None of the mounting goes through the outside walls of the exit section or otherwise impedes the flow.

The nuts holding the top and bottom brackets to the top and bottom support brackets and the nuts holding the top and bottom support brackets to the threaded rod are loosened in order to adjust the angle of the frame relative to the flow. Once the angle is set, these nuts are all tightened securely, to prevent movement of the frame due to the wind loading. For additional rigidity, a wooden leg is added between the downstream corner of the frame and the laboratory floor. A thin rope is used to tie the downstream corners of the frame to a brace on the wall. Even with these supports, the frame vibrates at high wind speeds. However, these vibrations have a much lower frequency than the frequency of the produced noise. When additional damping is added to the plate by holding it by hand, the magnitude and frequency of the produced tones remain unchanged. Therefore, the noise is produced purely by the fluid dynamic mechanism and is not caused or enhanced by the vibration of the plate.

The angle of incidence of the plate with respect to the flow is varied in 5 degree increments from 0 to 40 degrees, with a 0 degree angle defined as flow which is parallel to the plate, as defined in Figure 13. The angle is set using a protractor with 0.5-degree increments. It is verified using geometric relationships. Using the width of the exit section $(711 \mathrm{~mm})$ and the length of the frame $(914.4 \mathrm{~mm})$, the length between the downstream corner of the frame and the opposite corner of the exit section can be determined for the desired angle of incidence. The angle is accurate to within 0.5 degrees. 
The top and bottom supports have a slot which runs along their horizontal length.

This slot is used for mounting test plates to the frame with screws. Flat-headed machine screws are used to avoid unwanted noise due to shedding from the screw heads. The screw heads are covered by masking tape during testing to further reduce this risk.

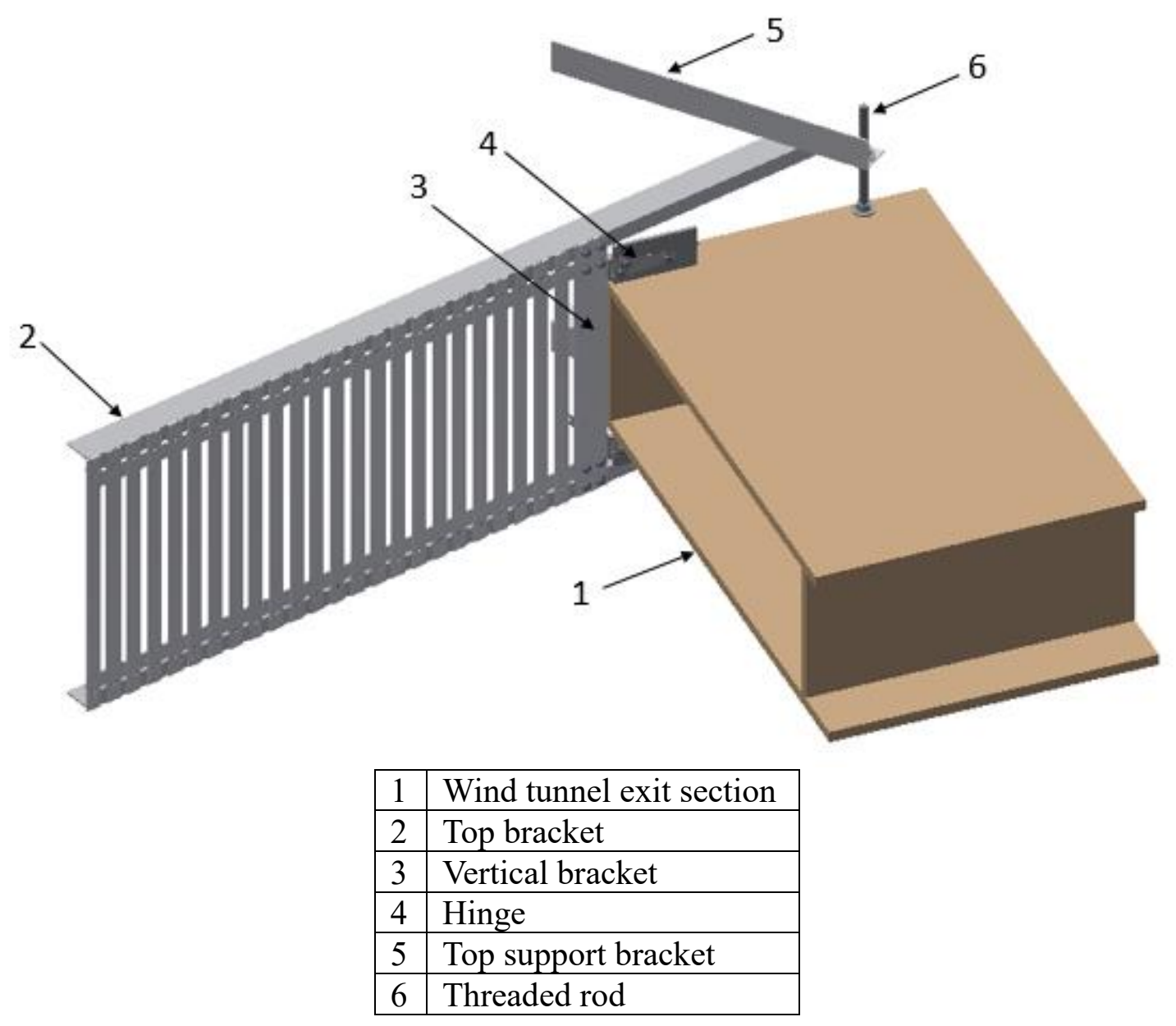

Figure 12: Test section consisting of a frame with rectangular slats mounted on hinges at the exit of an open-loop wind tunnel. 


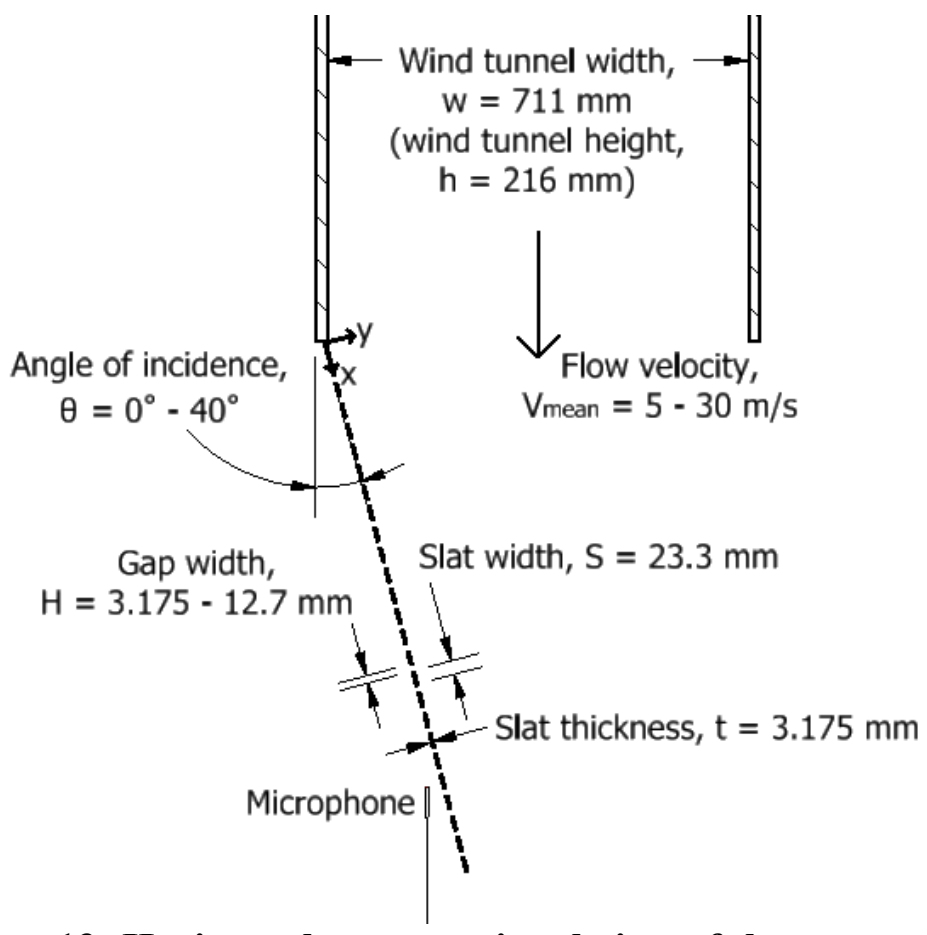

Figure 13: Horizontal cross-sectional view of the test section.

Two types of test plates are used in this study. First, a typical perforated plate is used to validate the experimental apparatus and methodology by comparing to the results from literature. This plate has a thickness of $1.59 \mathrm{~mm}(1 / 16$ inch $)$ and has holes with a diameter of $6.35 \mathrm{~mm}(1 / 4 \mathrm{inch})$ with a center-to-center spacing of $12.7 \mathrm{~mm}(1 / 2 \mathrm{inch})$. This corresponds to a solid width over thickness ratio, $S / t$, of 4 and a diameter over thickness ratio, $D / t$, of 4 . This plate is manufactured and supplied by Wellington Perforated Sheet \& Plate Inc. (Wellington, 2017). The holes are made with a press, which results in a different edge roundness on each side of the plate. The side of the plate which is pressed has a rounded edge, which is referred to as the "blunt" edge. The other side is referred to as the "sharp" edge. The plate is tested with both edges of the plate exposed to the flow. This yields different results for each side, which is discussed in Chapter 4: Results and 
Discussion. In general, it is found that this perforated plate produces tonal noise in the same range of Strouhal numbers for the same range of input conditions as what is found in the literature.

The second type of test plate is a two-dimensional simplification of a perforated plate. To simplify the geometry to two dimensions, a perforated plate is modelled by a series of rectangular Aluminum slats with an adjustable gap width between them. These slats are $23.29 \mathrm{~mm}$ (0.917 inches) wide by $3.175 \mathrm{~mm}$ (0.125 inches) thick by $431.8 \mathrm{~mm}$ (17 inches) long. This corresponds to a solid width over thickness ratio, $S / t$, of 7.33 , which was chosen to correspond with plate $\# 5$ of Feng from the literature (see Table 1) (Feng, 2012). The slats are machined to a consistent width from a 6061 Aluminum alloy sheet. All sides of the slats are filed, resulting in sharp edges. The sharpness of the slat edges is similar to the "sharp" edge of the perforated plate. The individual slats experience some vibration at high wind speeds. However, similar to the vibrations of the frame, these vibrations have a much lower frequency than the frequency of the flow-induced noise. When this vibration is reduced by holding the slats by hand, the magnitude and frequency of the produced tones remain unchanged. Therefore, vibration of the slats has no effect on the produced noise.

In this two-dimensional model, the hole diameter, $D$, of a perforated plate is replaced by the effective diameter, $d_{e}$, which is defined in equation (3). The slats are securely fixed to the frame with a flat-head machine screw at each end. The gap width is adjusted by changing the position of the slats along the length of the frame. Each slat is positioned individually starting at the upstream end of the frame, using a caliper to set the gap width. The following three gap widths are tested: $3.175 \mathrm{~mm}(1 / 8 \mathrm{inch}), 6.35 \mathrm{~mm}(1 / 4$ 
inch), and $12.7 \mathrm{~mm}(1 / 2 \mathrm{inch})$. This corresponds to a gap width over thickness ratio, $H / t$, of 1,2 , and 4 , respectively.

\subsection{Acoustic Measurements}

A G.R.A.S. 40BP 1/4-inch microphone, G.R.A.S. 26AC preamplifier, and G.R.A.S. 12AA power supply are used to measure the acoustic pressure fluctuations downstream of the experimental apparatus. The microphone is a high-precision condenser microphone designed for high sound pressure levels and high frequencies. A G.R.A.S. RA0022 1/4-inch nose cone is used on the microphone to reduce the amount of background noise due to the turbulence of the flow. A National Instruments 9233 data acquisition device is used to record the output of this microphone system. The signal is outputted to a computer, where a MATLAB program records the input signal and displays it.

The microphone is calibrated using a G.R.A.S. $42 \mathrm{AB}$ pistonphone. The pistonphone emits a single frequency sound at $1000 \mathrm{~Hz}$ and a sound pressure level of $114 \mathrm{~dB}(10 \mathrm{~Pa})$. A calibration constant is applied to the MATLAB code to convert the voltage output of the microphone system to the correct sound pressure level. For each data point, the voltage signal is recorded for 5 minutes at a sampling rate of 50,000 $\mathrm{Hz}$. For each 1-second time interval, the signal is windowed using a Hanning window, and the Fast Fourier transform is calculated to obtain the frequency spectrum of the signal. The average of these 300 spectra is calculated to obtain the final frequency spectrum.

For all the acoustic measurements, the microphone is positioned at the midpoint of the height of the wind tunnel exit section in the vertical direction (Z-direction in Figure 13). 
It is moved to different positions in the $X$ and $Y$ directions. Unless otherwise stated, all the acoustic measurements are taken when the microphone is positioned $25.4 \mathrm{~mm}(1 \mathrm{inch})$ from the back of the slats (negative $Y$-direction in Figure 13) and $762 \mathrm{~mm}$ (30 inches) downstream along the length of the plate ( $X$-direction in Figure 13). This is $152.4 \mathrm{~mm}$ (6 inches) from the downstream edge of the plate in the negative $X$-direction. The microphone in Figure 13 is shown at its correct scale at this location. As will be shown in Chapter 4: Results and Discussion, the tonal noise produced by the plates increases in the streamwise direction up to its maximum, then decreases near the downstream end of the plate. The chosen microphone location is found to be the location where the flow along the plate is fully developed and the tonal noise which is emitted is at its maximum.

When the microphone is moved further away from the plate in the $Y$-direction, the signal to noise ratio decreases. The magnitude of the measured tonal peaks decreases and the noise from other sources in the room become relatively greater. Therefore, the microphone is kept close to the back of the plate at a $Y$-position of negative $25.4 \mathrm{~mm}$ for all acoustic measurements. Because of the background noise in the room, the sound power level cannot be accurately calculated. Instead, the results are given as sound pressure at specific microphone locations.

For the acoustic results, the frequency is expressed using the dimensionless Strouhal number, $S t$, defined in equation (2). For a perforated plate, the characteristic dimension, $d$, is defined as the hole diameter, $D$. For the rectangular slats, it is defined as the effective diameter, $d_{e}=(4 H / \pi)$, where $H$ is the gap width. This factor of $(4 / \pi)$ is applied to account for the fact that a gap with a width $H$ acts similarly to a round hole with a diameter $4 H / \pi$, 
as shown by Bruggeman and discussed in Section 2.5 (Bruggeman, 1987). This definition of $d_{e}$ is found to give good agreement for the $S t$ values between the circular and the rectangular holes.

For most of the acoustic results, the magnitude of the microphone signal is expressed as the normalized sound pressure, $P^{*}$, where $P$ is the rms amplitude of the pressure fluctuation, $\rho$ is the density of air at room temperature, and $V_{\text {mean }}$ is the mean flow velocity:

$$
P^{*}=\frac{P}{\frac{1}{2} \rho * V_{\text {mean }}^{2}}
$$

\subsection{Particle Image Velocimetry Measurements}

Two-dimensional phase-locked particle image velocimetry (PIV) is used to obtain detailed measurements of the flow field in front of, behind, and between the slats. The light for the PIV system is provided by a $532 \mathrm{~nm}$ New Wave Solo 120XT pulsed Nd:YAG laser. A 12-bit Power View 4MP CCD camera with a Nikon AF Nikkor $50 \mathrm{~mm}$ lens is used to capture the images. An optical bandpass filter with a bandwidth of $10 \mathrm{~nm}$, centered at the laser frequency of $532 \mathrm{~nm}$, is used at the end of the camera lens to allow only light from the laser to enter the camera. The timing of the camera images and laser pulses is synchronized using a TSI LaserPulse 610035 synchronizer. A Laskin nozzle oil droplet generator is used to seed the flow with bis (2-ethylhexyl) sebacate particles, with a particle diameter of $1 \mu m$.

The camera is positioned above the test section and focused on the horizontal laser sheet. The camera is spatially calibrated by taking an image of a ruler placed in the field of 
view, level with the laser sheet. The number of pixels between defined points on the ruler are measured and the pixel size is calculated in units of $\mu \mathrm{m} /$ pixel. This calibration is repeated every time the camera or laser are moved. For the images shown in this thesis, the average pixel size is between 15 and $16 \mu \mathrm{m}$, depending on the distance of the camera from the laser sheet.

Insight $4 \mathrm{G}$ software is used to process all the PIV images using the Classic PIV algorithm. A 24-by-24 pixel interrogation region is used. For a pixel size of $15.7 \mu m$, this corresponds to a 376.8-by-376.8 $\mu \mathrm{m}$ interrogation region. The total field of view of the camera is 2048-by-2048 pixels. Therefore, the field of view is approximately $32-b y-32 \mathrm{~mm}$ in size and consists of 85-by-85 interrogation regions.

The time step between the two camera images is calculated based on the size of the interrogation region and the maximum flow velocity in the field of view, using equation (5) below. In order to accurately track the particles from one frame to the next, the maximum particle displacement should be approximately one quarter of the interrogation region. Using this processing scheme, pixel size, and time step, this produces processed images with a vector validation rate of $98-99 \%$.

$$
\Delta T=\frac{\frac{1}{4} *(\text { interrogation region size })}{V_{\max }}
$$

The PIV images are phase-locked using the same microphone system as was used for the acoustic measurements. The microphone is positioned downstream of the gap which is being looked at, just outside the field of view of the camera. A bandpass filter is used with the microphone system to filter out everything except for the largest tonal peak. This 
filter is centered at the peak frequency of the dominant tone. The bandwidth is adjusted between $500 \mathrm{~Hz}$ and $1000 \mathrm{~Hz}$ based on the width of the main peak. In Figure 14, the FFT of the microphone signal is shown, with a large peak centered at $2720 \mathrm{~Hz}$. In this case, the filter has a bandwidth of $683 \mathrm{~Hz}$, with a high pass of $2400 \mathrm{~Hz}$ and a low pass of $3083 \mathrm{~Hz}$, to remove all frequency components except for this peak.

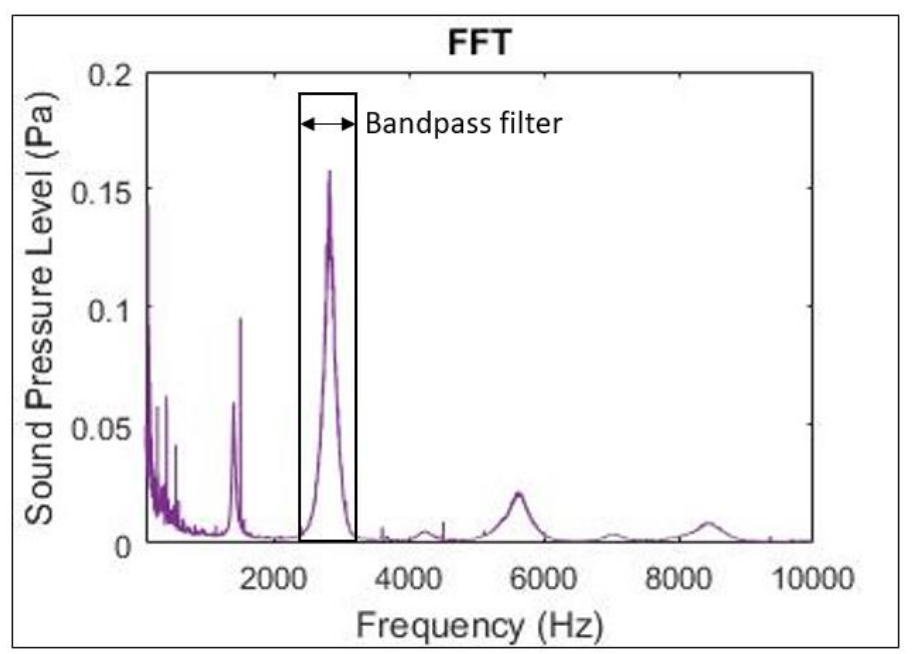

Figure 14: FFT of the microphone signal showing the limits of the bandpass filter centered at the dominant tonal frequency.

During PIV testing, a gain is applied to increase the voltage of the microphone signal. Using this filtered and amplified signal, a trigger system is used to trigger the PIV system to take images at a defined point in the acoustic cycle. This trigger sends a pulse when the signal crosses the 0 Volt point in the negative direction. Considering the period of the filtered signal, a time delay is applied to capture images at defined points in the cycle. Images are taken at 8 different points in the cycle, corresponding to phases of $0^{\circ}, 45^{\circ}, 90^{\circ}$, $135^{\circ}, 180^{\circ}, 225^{\circ}, 270^{\circ}$, and $315^{\circ}$. This is shown in Figure 15 . This blue line in this figure is the filtered and amplified microphone signal. After filtering, the signal is nearly 
sinusoidal. The trigger signal is the red pulse at the zero crossing of the filtered signal. The red X's denote the 8 phases at which images are taken.

For each phase angle, 200 PIV images are taken. The average of these 200 images is calculated to obtain the average flow field at that particular phase angle. In addition, 200 images are taken at random phases, in order to study the mean flow field.

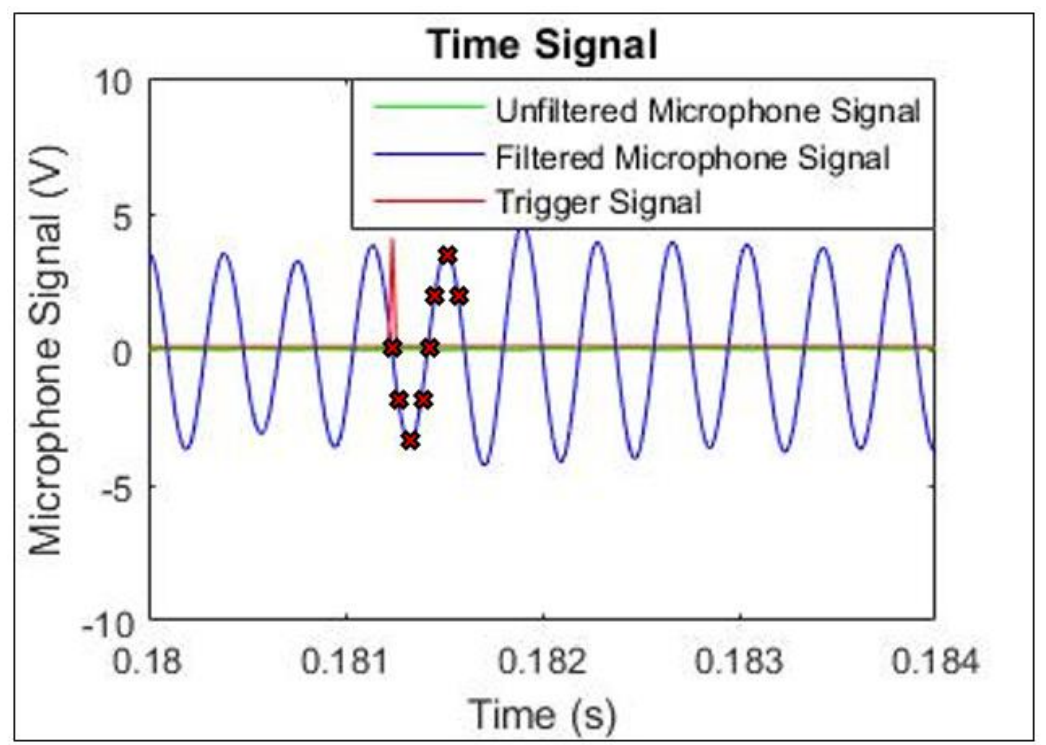

Figure 15: Time signal of the unfiltered and filtered microphone signal showing the trigger location. The red $X$ 's denote the 8 points in the acoustic cycle at which PIV images are taken: $0^{\circ}, 45^{\circ}, 90^{\circ}, 125^{\circ}, 180^{\circ}, 225^{\circ}, 270^{\circ}$, and $315^{\circ}$.

In order to minimize reflections of the laser light from the Aluminum surfaces when taking PIV images, all metal surfaces of the slats are covered in a thin black vinyl tape. The black surface of this tape reflects less of the laser light, allowing the flow field to be resolved closer to the surfaces of the slats. This also prevents damage to the camera due to overexposure. Like the slats themselves, the tape has a smooth surface. It has a thickness of $0.089 \mathrm{~mm}$ (0.0035 inches), which increases the thickness of the slats from $3.175 \mathrm{~mm}$ (0.125 inches) to $3.353 \mathrm{~mm}$ (0.132 inches). For a gap width of $6.35 \mathrm{~mm}$ (1/4 inches), this 
decreases the $H / t$ ratio from 2 to 1.89 . Taping the slats is found to have a small effect on the magnitude and frequency of the produced tones, as shown in Section 4.3.5: Effect of streamwise position.

Because the laser sheet cannot pass through the slats, different laser positions are used to capture the area in front of and behind the slats. First, the laser is positioned in front of the slats, at $Y=0.5$ meters, where $Y$ is defined in Figure 13, and 0.5 meters is the focal length of the laser lens. Second, the laser is positioned behind the slats, at $Y=-0.5$ meters. This allows both sides of the slats to be captured. With both laser positions, the area between the slats can also be seen. The laser sheet is focused on a single gap at a time. Different gaps are chosen to show the development of the flow along the length of the plate. Specifically, the $6^{\text {th }}, 12^{\text {th }}, 18^{\text {th }}$, and $24^{\text {th }}$ gaps are looked at for a gap width of $6.35 \mathrm{~mm}$ and an angle of incidence of 15 degrees. The laser sheet is aligned level with the $X$ - $Y$ plane, at a height in the $Z$-direction equal to the midpoint of the wind tunnel exit section.

The PIV results are expressed as dimensionless terms, $X^{*}$ and $Y^{*}$. The $X$ distance is expressed as a fraction of the gap width, $H$. The $Y$ distance is expressed as a fraction of slat thickness, $t$.

$$
\begin{gathered}
X^{*}=\frac{X}{H} \\
Y^{*}=\frac{Y}{t}
\end{gathered}
$$

The velocity magnitude is expressed as a fraction of the mean velocity, where $V$ is the velocity magnitude and $V_{\text {mean }}$ is the mean velocity exiting the wind tunnel. The velocity 
magnitude consists of the $X$ and $Y$ components of the velocity field only. The out of plane $Z$ component is assumed to be negligible for this two-dimensional model.

$$
V^{*}=\frac{V}{V_{\text {mean }}}
$$

The vorticity is expressed as dimensionless vorticity, $\omega^{*}$, where $\omega$ is the vorticity and $H$ is the gap width.

$$
\omega^{*}=\frac{\omega * H}{V_{\text {mean }}}
$$

The vorticity is the curl of the velocity field and is calculated using the velocity field data obtained from the PIV images. The following equation is used:

$$
\omega=\vec{\nabla} \times \vec{V}=\frac{\partial v}{\partial x}-\frac{\partial u}{\partial y}
$$

Individual coherent vortices are identified in the vorticity field using the $d_{2}$ parameter (Vollmers, 2001). The $d_{2}$ parameter is defined in equation (11) below, where $G$ is the gradient matrix, and $u$ and $v$ are the $x$ and $y$ components of the velocity field. Negative values of $d_{2}$ indicate vortices, with greater negative values indicative of stronger vortices. This parameter does a better job of identifying individual vortices than just looking at the vorticity itself. In particular, it does not generally identify steady boundary layers and shear layers as vortices, despite the fact that they may have high vorticity. Vollmers reviewed the $d_{2}$ parameter along with several other methods of vortex identification, and concluded that it was the best indicator of vortices, but has two drawbacks. The first drawback of this method is that it requires smooth velocity field data to accurately calculate the gradients. 
In this thesis, it is applied to PIV velocity field data which is the average of 200 images, resulting in a smooth field. A second drawback is that calculation of the derivatives can be time consuming, which is also not a concern for the purposes of this work. In the phaselocked PIV images, a single contour of the $d_{2}$ parameter is overlaid onto the vorticity field to identify and track the movement of vortices.

$$
d_{2}=(\operatorname{trace} G)^{2}-4 \operatorname{det} G=\left(\frac{\partial u}{\partial x}+\frac{\partial v}{\partial y}\right)^{2}-4\left(\frac{\partial u}{\partial x} * \frac{\partial v}{\partial y}-\frac{\partial u}{\partial y} * \frac{\partial v}{\partial x}\right)
$$

Using the velocity field data from the PIV results, the momentum thickness of the free shear layer is calculated using equation (12) below, where $u$ is the local velocity in the $X$-direction and $U$ is the free stream velocity.

$$
\Theta=\int_{0}^{\infty} \frac{u}{U}\left(1-\frac{u}{U}\right) d y
$$

Because the velocity field data from the PIV consists of discrete pixels, equation (12) is discretized in equation (13), where $u_{i}$ is the velocity at a given data point, $U$ is the free stream velocity, and $\left(y_{i}-y_{i-1}\right)$ is the distance between successive data points.

$$
\Theta=\sum_{i=0}^{\infty} \frac{u_{i}}{U}\left(1-\frac{u_{i}}{U}\right)\left(y_{i}-y_{i-1}\right)
$$

The momentum thickness is used to calculate the Strouhal number based on momentum thickness, $S t_{\Theta}$. As discussed in the Chapter 2: Literature Review, $S t_{\Theta}$ is used to investigate the stability of the free shear layer. 


\subsection{Geometric Inconsistencies}

There are several sources of error and uncertainty related to the experimental apparatus and measurement techniques. The main source is caused by inconsistencies in the test section. Because the slats are relatively thin at $3.175 \mathrm{~mm}(1 / 8 \mathrm{inch})$ and are required to span a distance of $330.2 \mathrm{~mm}$ (13 inches), they are prone to twisting and bending. This can change the effective angle, gap width, and thickness of the gap. Figure 16(a) illustrates the case of grazing flow over two properly aligned slats. Figure 16(b) shows the case where the upstream slat is twisted in the clockwise direction, and Figure 16(c) shows the case where the upstream slat is bent, causing it to shift downward relative to the downstream slat. In the second two cases, the offset between the two slats changes the effective geometry of the gap. This leads to inconsistent results from one gap to the next. This is seen in the acoustic results in Section 4.3.5: Effect of streamwise position, and in the PIV results in Section 4.4.2.3: Effect of streamwise position.

As a results of the misalignment of some of the slats, it is difficult to draw conclusions based on the results from a single gap. It is important to look at the overall trends over multiple gaps. For the PIV measurements, care was taken to make sure that the two slats which were focused on were correctly aligned. 
a)

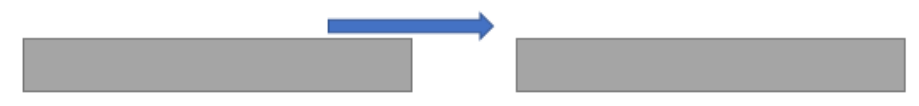

b)

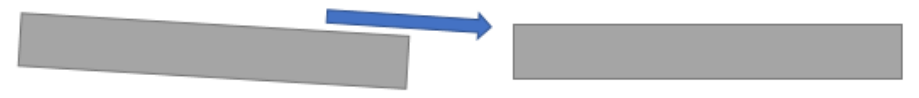

c)

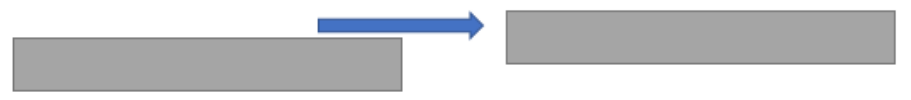

Figure 16: Cross-sectional view of a series of two slats: (a) correctly aligned slats, (b) upstream slat twisted, (c) upstream slat bent causing offset.

An analysis of additional sources of error in the experimental procedure is found in Appendix A: Uncertainty Analysis. 


\section{CHAPTER 4 RESULTS AND DISCUSSION}

\subsection{Introduction}

The experimental results are presented in three main parts. First, the acoustic results for the perforated plate are shown. These results are compared to those from literature to validate the experimental apparatus and methodology. Second, the acoustic results are shown for the two-dimensional slats. Results are discussed for different angles of incidence, flow velocities, gap widths, and streamwise positions. These results show the conditions which are favorable to the production of tonal noise and the dependence on various input parameters. Third, particle image velocimetry results are shown for flow over the slats. The purpose of the PIV measurements is to understand the physical mechanism which leads to the production of tonal noise by the slats, and in extension by a perforated plate. The focus of the PIV results is on the gap width and angle which were found in the acoustic results to produce the most well-defined tones. In addition, results are discussed for different angles of incidence, flow velocities, and streamwise positions.

\subsection{Acoustic Results - Perforated Plate}

To validate the experimental apparatus, the acoustic response of a perforated plate is measured and compared to the results from literature. In Figure 9, Feng showed that a perforated plate with a thickness of $1.5 \mathrm{~mm}$ and a hole diameter of $8 \mathrm{~mm}$ produced tonal 
noise in the $2500 \mathrm{~Hz}$ frequency band when exposed to flow at an angle of incidence of $15^{\circ}$ and a velocity of $15 \mathrm{~m} / \mathrm{s}$ (Feng, 2012). The perforated plate tested for validation has a thickness of $1.59 \mathrm{~mm}$ and a hole diameter $6.35 \mathrm{~mm}$ and is tested at the same angle of incidence and flow velocity as the results of Feng. The frequency response of this plate is plotted in Figure 17. In this figure, the black lines are the results of Feng for various hole diameters and the colored lines are the results of this research. For both the blunt edge and the sharp edge of the plate exposed to the flow, there is a tonal peak in the $2500 \mathrm{~Hz}$ frequency band. This agrees well with the $D=8 \mathrm{~mm}$ plate of Feng, which is the closest in geometry to the $D=6.35 \mathrm{~mm}$ plate which was tested.

The results of Feng are expressed as A-weighted sound power level found using a rotating microphone in an anechoic room. The results of this research are expressed as Aweighted sound pressure level at a single microphone location in an open room. The location of the microphone is $25.4 \mathrm{~mm}(1 \mathrm{inch})$ from the back of the slats in the $Y$-direction and $762 \mathrm{~mm}$ (30 inches) downstream along the length of the plate in the $X$-direction. This is $152.4 \mathrm{~mm}$ (6 inches) from the downstream edge of the plate in the negative $X$-direction (The $X-Y$ axis is defined in Figure 13). These measurements are also subject to reflections of sound and background noise. Because of the different levels of background noise, different experimental apparatuses, and different microphone locations, the magnitudes of the sound levels are not directly comparable between the two studies. Only the frequencies are directly comparable. 


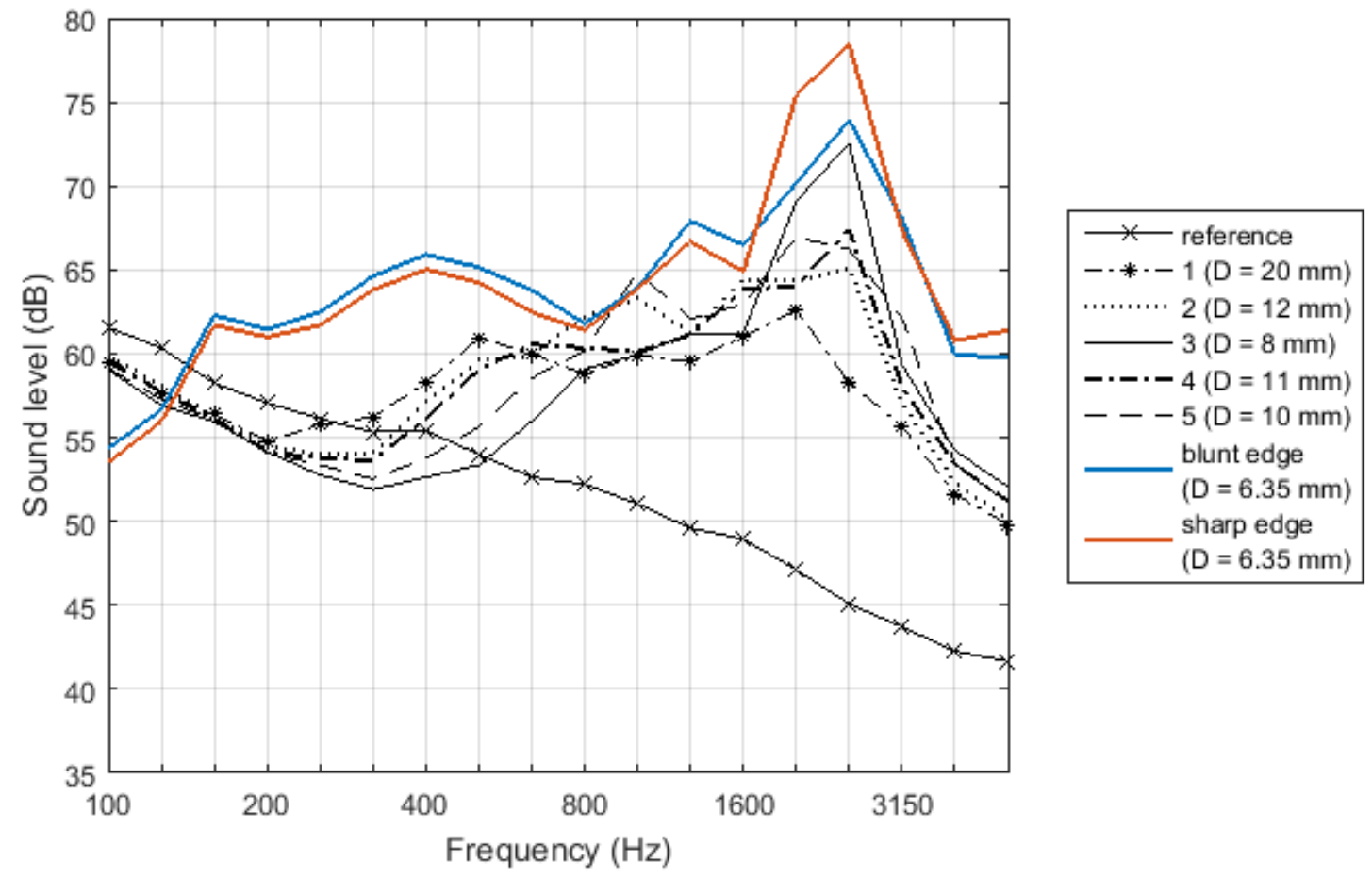

Figure 17: Frequency spectra for a perforated plate with an angle of incidence of $15^{\circ}$ and a flow velocity of $15 \mathrm{~m} / \mathrm{s}$. The black lines are the results of Feng for a hole diameter of $8 \mathrm{~mm}$ to $20 \mathrm{~mm}(D / t=5.3$ to 13.3), expressed in A-weighted sound power level (dB) (Feng, 2012). The colored lines are the results of this research, with a hole diameter of $6.35 \mathrm{~mm}(D / t=4)$, expressed in A-weighted sound pressure level $(\mathrm{dB})$. The blue line is for the blunt edges exposed to the flow and the red line is for the sharp edges exposed to the flow.

In Figure 10, Feng showed that as the flow velocity increases, the frequency of the tones increase linearly (Feng, 2012). The overall sound power level also increased with increasing flow velocity due to increasing energy in the flow. In Figure 18, the results of this research are compared with the results of Feng concerning the effect of flow velocity. The black lines are the results of Feng for a plate with a thickness of $1.5 \mathrm{~mm}$ and a hole diameter of $8 \mathrm{~mm}$ at an angle of incidence of $15^{\circ}$ and a variable flow velocity. The colored lines are the results of this research at the same angle of incidence using the validation plate 
$(t=1.59 \mathrm{~mm}, D=6.35 \mathrm{~mm})$. Both sets of results show the frequency of the tones increasing linearly with increasing flow velocity. While the results of Feng stop at $15 \mathrm{~m} / \mathrm{s}$, the results of this research show that the trend continues up to a velocity of $30 \mathrm{~m} / \mathrm{s}$.

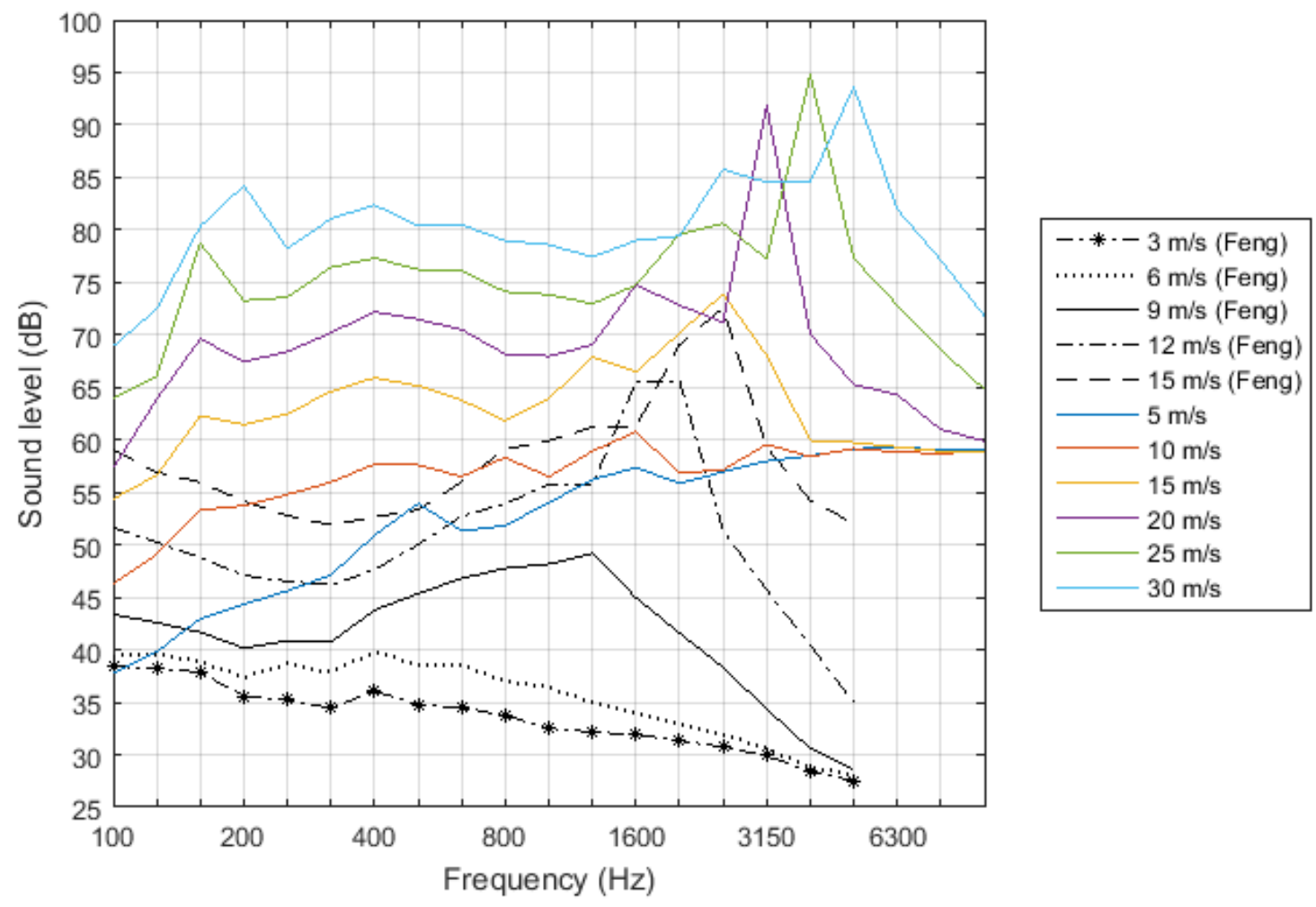

Figure 18: Influence of the wind velocity on the frequency spectrum for a perforated plate at an angle of incidence of $15^{\circ}$. The black lines are the results of Feng for a hole diameter of $8 \mathrm{~mm}(D / t=5.3)$, expressed in A-weighted sound power level (dB) (Feng, 2012). The colored lines are the results of this research with a hole diameter of 6.35 $m m(D / t=4)$, expressed in A-weighted sound pressure level (dB). The blunt edges of the plate are exposed to the flow.

In Figure 17 and Figure 18, a sample of the results of Feng have basically been reproduced using a perforated plate with an approximately similar geometry. Tones are observed in the same range of frequencies for the same range of angles and flow velocities. 
When the results of the acoustic measurements for the perforated plate are expressed in narrow band frequencies, it is observed that tones are excited at two different frequencies. Figure 19 shows the acoustic results for the perforated plate at an angle of incidence of $15^{\circ}$ and a varying flow velocity. The results are plotted in $1 \mathrm{~Hz}$ band frequencies, with the frequency converted to Strouhal number and the sound pressure level converted to normalized pressure. Because the frequency of the tones increases linearly with increasing flow velocity, the peaks at different flow velocities collapse to a single Strouhal number.

The $15 \mathrm{~m} / \mathrm{s}$ case in Figure 19 shows the same data as the colored lines in Figure 17, While the dominant peak can be seen at $S t=0.96$, another smaller peak is also visible at $S t$ $=0.55$. When the flow velocity is increased to $20 \mathrm{~m} / \mathrm{s}$, the lower frequency becomes the dominant mode for the sharp edge of the plate exposed to the flow, while the higher frequency remains the dominant mode for the blunt edge of the plate exposed to the flow. Because both modes are present for both plate orientations, this is not the case of a single mode shifting to a higher or lower frequency depending on the curvature of the hole edge. The situation remains the same at a velocity of $25 \mathrm{~m} / \mathrm{s}$ and $30 \mathrm{~m} / \mathrm{s}$, though the magnitude of the peaks decreases. The idea that the same plate produces different acoustic results depending on whether the sharp or the blunt edge is exposed to the flow agrees with the results of Blinet et al. (2015), as shown in Figure 11. 


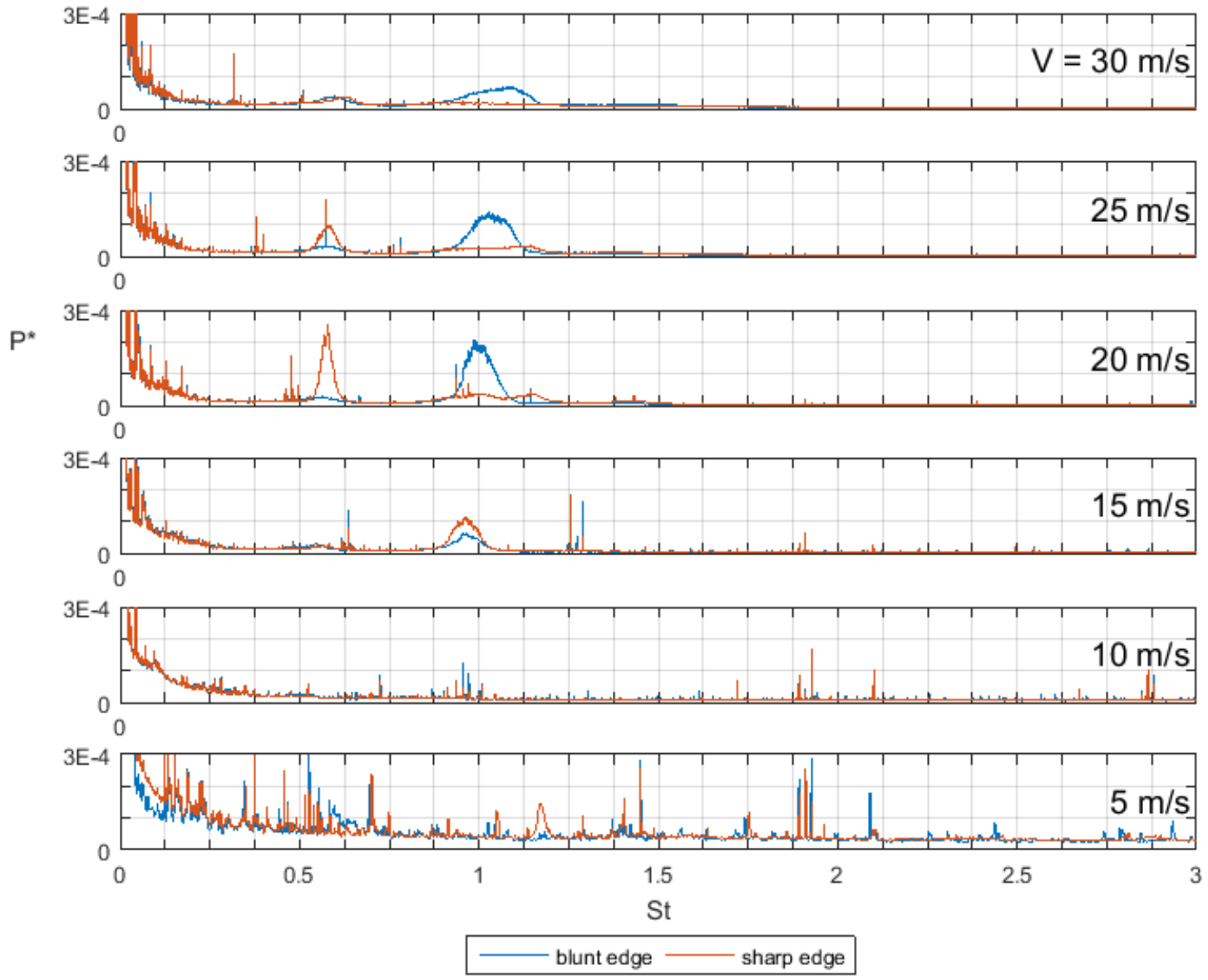

Figure 19: Frequency spectra for a perforated plate with a hole diameter of $6.35 \mathrm{~mm}$, an angle of incidence of 15 degrees, and a flow velocity of 5 to $30 \mathrm{~m} / \mathrm{s}$. The red line is for the sharp edge of the perforations exposed to the flow, while the blue line is for the blunt edge exposed to the flow.

The results are similar to Figure 19 for an angle of incidence of 20 degrees and 25 degrees. There is a mode at $S t=0.58$ to 0.66 , which is excited when the sharp edge of the plate is exposed to the flow. The second mode is at $S t=1.00$ to 1.08 and is excited when the blunt edge of the plate is exposed to the flow. The Strouhal number of the tones increases slightly with increasing angle of incidence. This may be due to a slight increase in the flow velocity along the surface of the plate due to a decrease of the flow area. At $\theta=$ 
$10^{\circ}$ and $\theta=30^{\circ}$, the tones are present, but are much weaker than for the range $\theta=15^{\circ}$ to $25^{\circ}$. For angles smaller than $10^{\circ}$ or larger than $30^{\circ}$, the noise is broadband only. For the frequency spectra for an angle of incidence of $\theta=0^{\circ}$ to $40^{\circ}$, see Appendix B.1: Acoustic Results - Perforated Plate.

The dependence on the angle of incidence is shown in Figure 20 for a mean flow velocity of $20 \mathrm{~m} / \mathrm{s}$. It is clear from this figure that there is a preferred angle of incidence at which the plate produces tonal noise. For this plate, the preferred angle is $\theta=15^{\circ}$ to $25^{\circ}$.

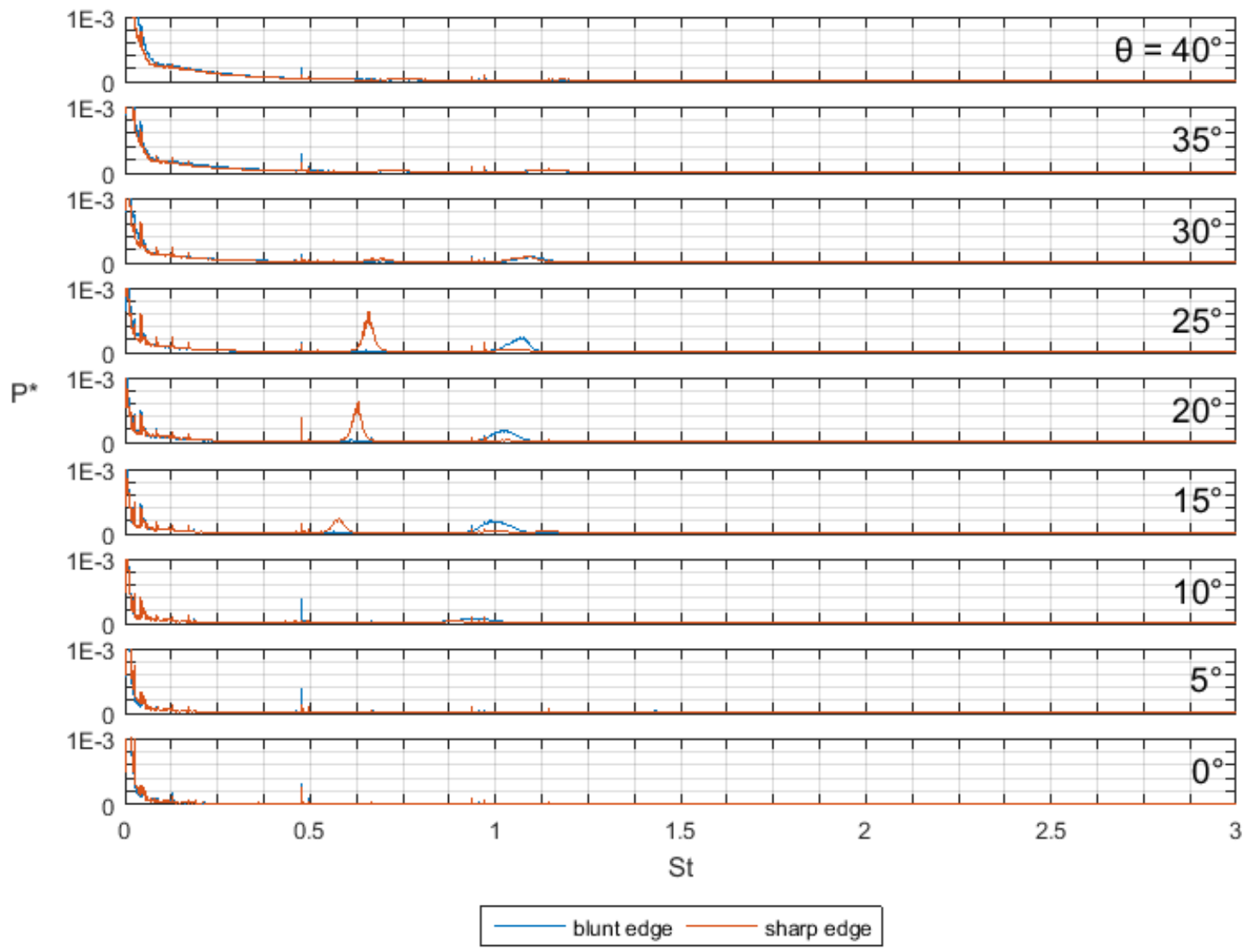

Figure 20: Frequency spectra for a perforated plate with a hole diameter of $6.35 \mathrm{~mm}$, a flow velocity of $20 \mathrm{~m} / \mathrm{s}$, and an angle of incidence of 0 to 40 degrees. The red line is for the sharp edge of the perforations exposed to the flow, while the blue line is for the blunt edge exposed to the flow. 
The results for the blunt edge of the plate exposed to the flow give good agreement with the results of Feng (2012) and Blinet et al. (2015) from the literature. The fact that different results are produced when the sharp edge of the plate is exposed to the flow agrees with the findings of Blinet et al.

\subsection{Acoustic Results - 2D Model}

\subsubsection{Comparison with literature}

The use of a two-dimensional model to study the flow over perforated plates is validated by comparing the results to those in the literature. In Figure 21, the results of Feng (2012) are plotted in black and the results of this research are plotted as colored lines. For the present $D=6.35 \mathrm{~mm}$ perforated plate, a tonal peak was measured at a frequency of 2500 $H z$, as shown by the red and blue lines. With the gap width set to $H=6.35 \mathrm{~mm}$, the twodimensional model produced a tone at a frequency of $2000 \mathrm{~Hz}$.

The Strouhal number is calculated for each of these peaks using equation (2). For the perforated plate, the characteristic dimension, $d$, is $6.35 \mathrm{~mm}$, which gives $S t=1.058$. For the slats, $d=(4 / \pi) * 6.35 \mathrm{~mm}$, which gives $S t=1.078$. Therefore, the Strouhal number of the peaks for the slats is nearly identical to that produced by the perforated plate for the same input conditions. The tones for the slats are also produced at the same range of angles and flow velocities as the perforated plates. Therefore, the two-dimensional model appears to reproduce the same mechanism of tone generation as the perforated plates. While some three-dimensional complexities of the flow field may be lost by changing to the slats, it 
appears that this two-dimensional model is an effective simplification for studying the main features of the mechanism.

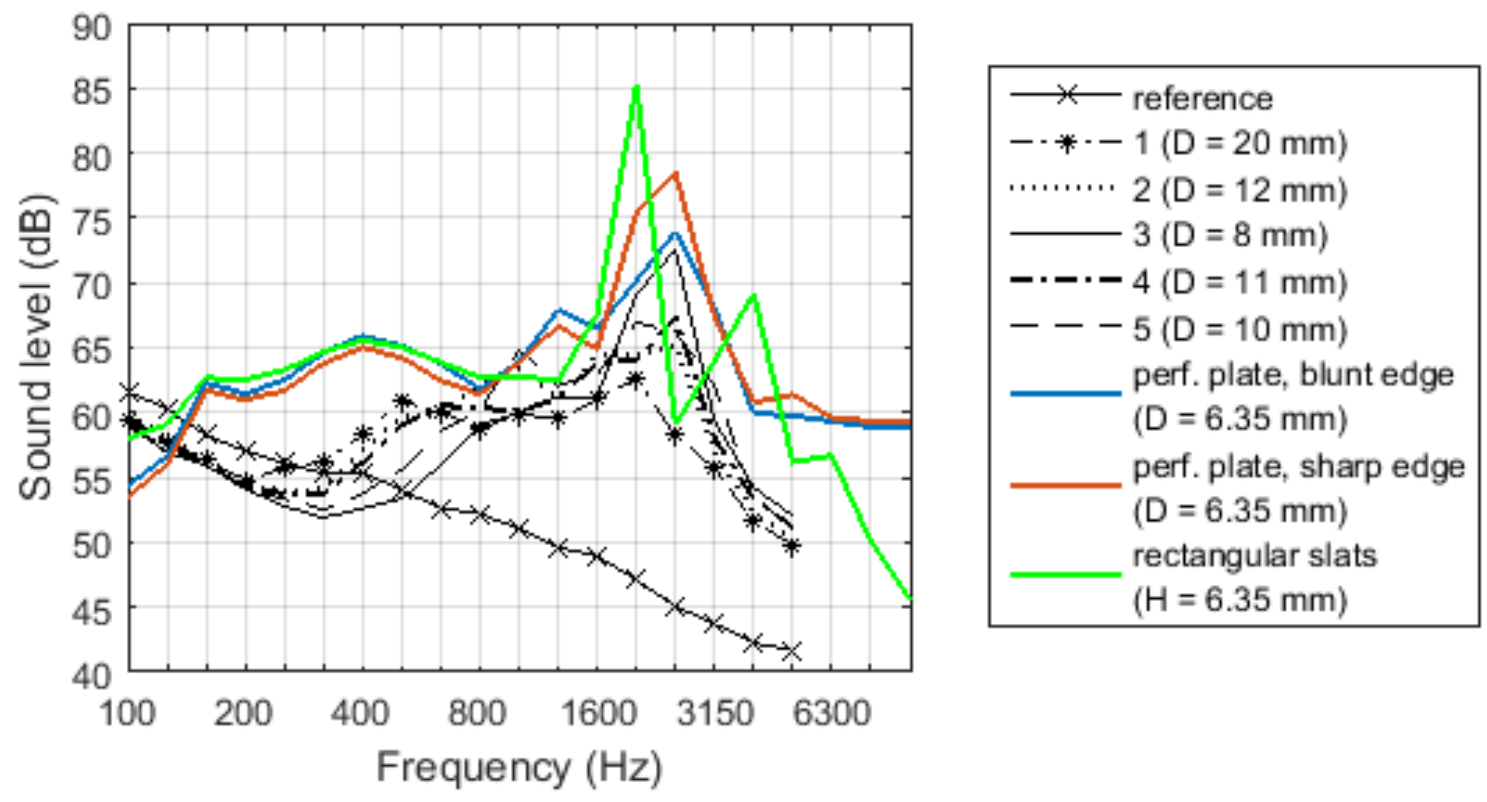

Figure 21: Frequency spectra for an angle of incidence of $15^{\circ}$ and a flow velocity of $15 \mathrm{~m} / \mathrm{s}$. The black lines are the results of Feng for a perforated plate with a hole diameter of $8 \mathrm{~mm}$ to $20 \mathrm{~mm}(D / t=5.3$ to 13.3), expressed in A-weighted sound power level (dB) (Feng, 2012). The red and blue lines are the results of this research, for a perforated plate with a hole diameter of $6.35 \mathrm{~mm}(D / t=4)$, expressed in A-weighted sound pressure level $(\mathrm{dB})$. The Green line is the results of this research, for the rectangular slats with a gap width of $6.35 \mathrm{~mm}(H / t=2)$.

In the following sections, the two-dimensional model is used to study the effect of angle of incidence, flow velocity, gap width, and streamwise position on the produced noise.

\subsubsection{Effect of angle of incidence}

When the angle of incidence of the plate with respect to the mean flow is varied for a constant gap width and wind velocity, tones are observed only for a specific range of 
angles. This range of angles depends on the gap width. For a gap width of $6.35 \mathrm{~mm}(1 / 4$ inch), tones are produced in the range of St of 1 to 1.3 for angles of incidence of 5 to 30 degrees. The strongest peak occurs for an angle of 15 degrees. The low frequency noise increases with increasing angle of incidence. This is because at larger angles, a greater amount of the flow passes through the gaps between the slats, producing a greater amount of turbulent noise downstream, near the location of the microphone.

Figure 22 shows the frequency spectrum for the case of a gap width of $6.35 \mathrm{~mm}$ $(H / t=2)$ and a flow velocity of $20 \mathrm{~m} / \mathrm{s}\left(\operatorname{Re}_{D}=8.4 \times 10^{3}\right)$. At an angle of incidence of 15 degrees, a large peak is observed at a Strouhal number of 1.15. In addition, a harmonic can be seen at a Strouhal number of 2.30 and a subharmonic can be seen at a Strouhal number of 0.57 . The Strouhal number of the peaks increases slightly with increasing angle of incidence. This was also observed for the perforated plate, and is likely due to a slight increase in the local flow velocity as the angle is increased. At larger angles, the flow is increasingly restricted by the plate, which leads to an acceleration of the flow along the length of the plate in the streamwise direction. As the angle is increased or decreased away from 15 degrees, the magnitude of the peaks decreases. An audible whistle can be heard for angles of approximately 5 to 30 degrees. At angles of 0 degrees or 35 degrees and greater, the noise is purely broadband and no tonal noise is observed, as can be seen in Figure 22 . 


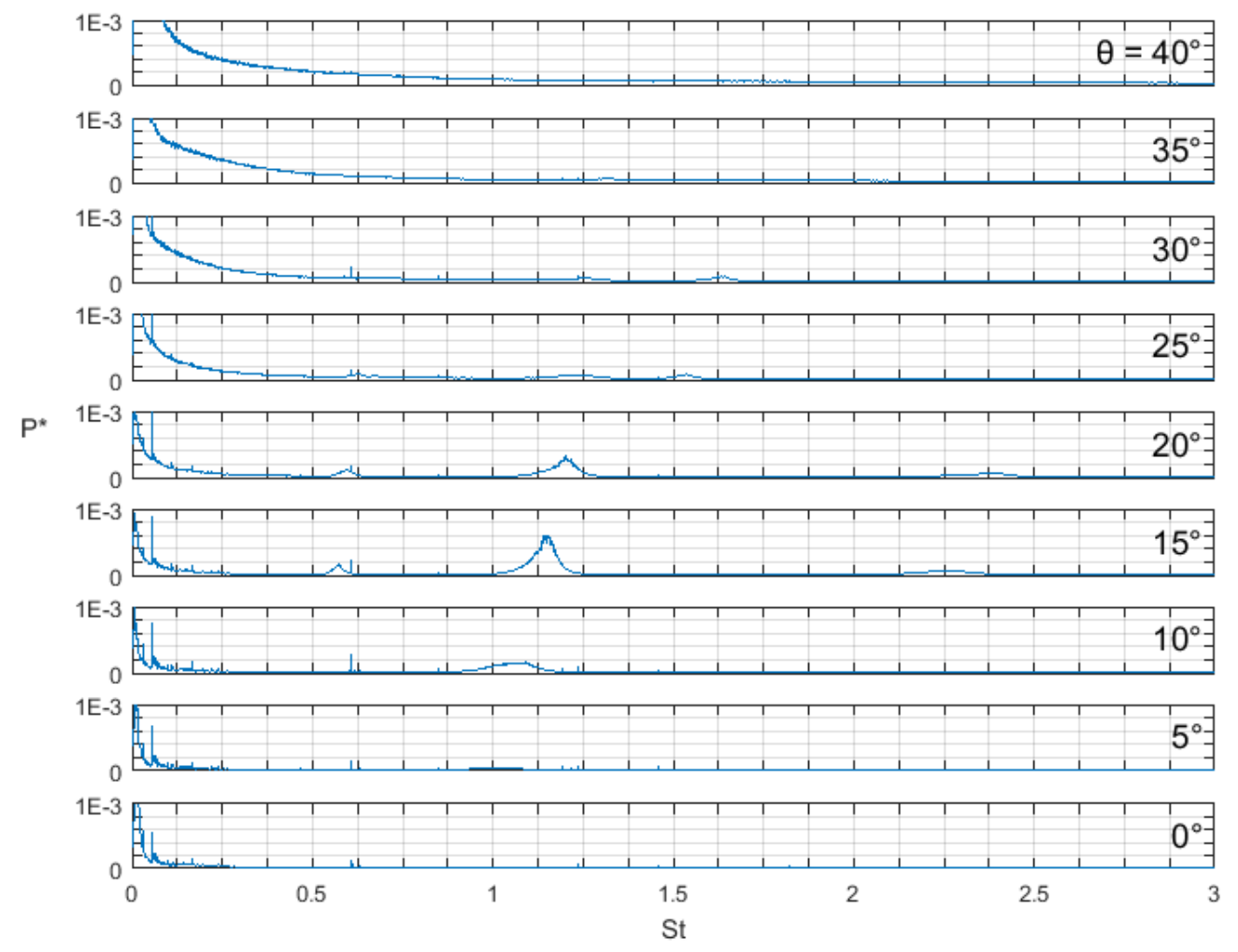

Figure 22: Frequency spectra for a gap width of $6.35 \mathrm{~mm}$, a flow velocity of $20 \mathrm{~m} / \mathrm{s}$, and an angle of incidence of 0 to 40 degrees. The Strouhal number, $S t$, is based on the effective gap width, $d_{e}$. This definition of $S t$ is used in all the analysis to follow.

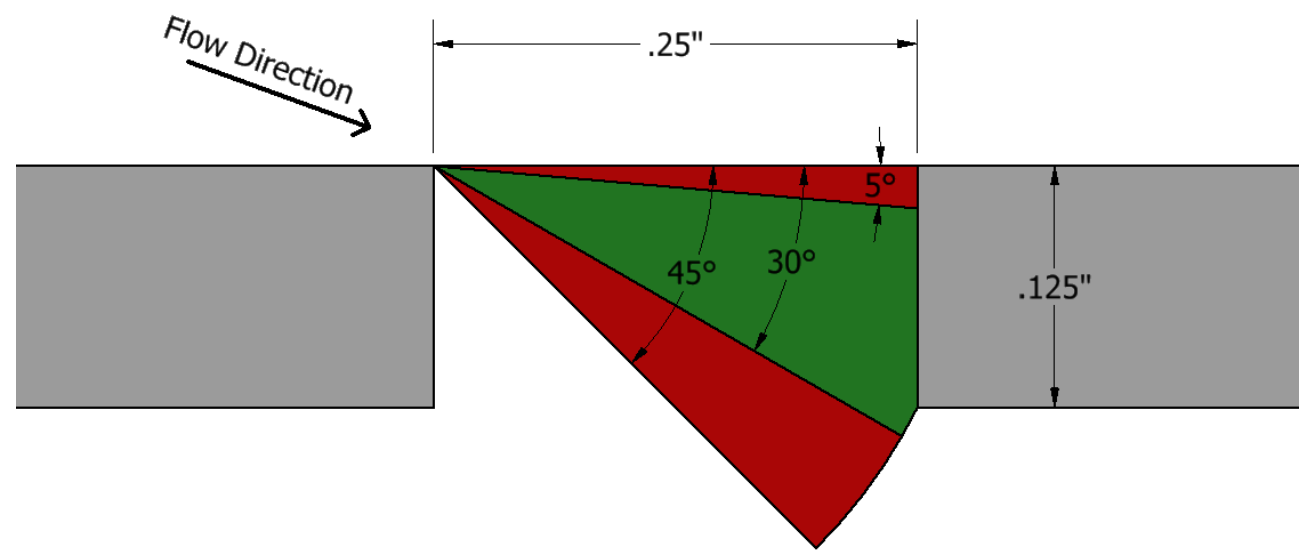

Figure 23: This illustration shows the angles of incidence favorable to the production of tonal noise for a gap width, $H=6.35 \mathrm{~mm}(0.25$ ") and an angle of incidence in the range $0^{\circ} \leq \theta \leq 45^{\circ}$. Green indicates that tones are produced, while red indicates that tones are not produced. Tones are produced for $\theta=5^{\circ}$ to $\theta=30^{\circ}$. 
Figure 23 illustrates the range of angles which produce tones for a gap width of 6.35 $\mathrm{mm}$. The green regions indicate the angles which produce tones, while the red regions indicate the angles which do not produce tones. This figure is based on the angle of the mean flow. The actual angle of the flow at the leading edge of the gaps is smaller than this, since the flow is directed along the surface of the plate as the flow develops along the plate's length.

A similar trend to the $H=6.35 \mathrm{~mm}$ case is observed for a gap width of $H=12.7 \mathrm{~mm}$ $(H / t=4)$, as shown in Figure 24 . For a flow velocity of $20 \mathrm{~m} / \mathrm{s}$, the largest tonal peak is observed at an angle of 15 degrees and a Strouhal number of 1.33. Harmonics are seen around a Strouhal number of 2.00 and 2.66, and a subharmonic is seen around a Strouhal number of 0.67 . The magnitude of this tone diminishes as the angle is increased or decreased away from 15 degrees, and remains audible in the range of 5 to 25 degrees. The range of angles which produce tones in the $H=12.7 \mathrm{~mm}$ case is shown in Figure 25 .

The frequency spectra for a gap width of $H=3.175 \mathrm{~mm}(H / t=1)$ and a flow velocity of $20 \mathrm{~m} / \mathrm{s}$ are shown in Figure 26. In this case, audible tones are measured for angles of incidence of 15 degrees and larger in the range of Strouhal numbers of 0.49 to 0.63 . It appears that these tones may continue for angles larger than 45 degrees, however, this is not measured due to limitations of the experimental apparatus. Similar to the gaps of 6.35 $\mathrm{mm}$ and $12.7 \mathrm{~mm}$, harmonics are also observed. However, the tones in this case are weak relative to those produced for the other gap widths. The range of angles which produce tones in the $H=3.175 \mathrm{~mm}$ case is shown in Figure 27 . 


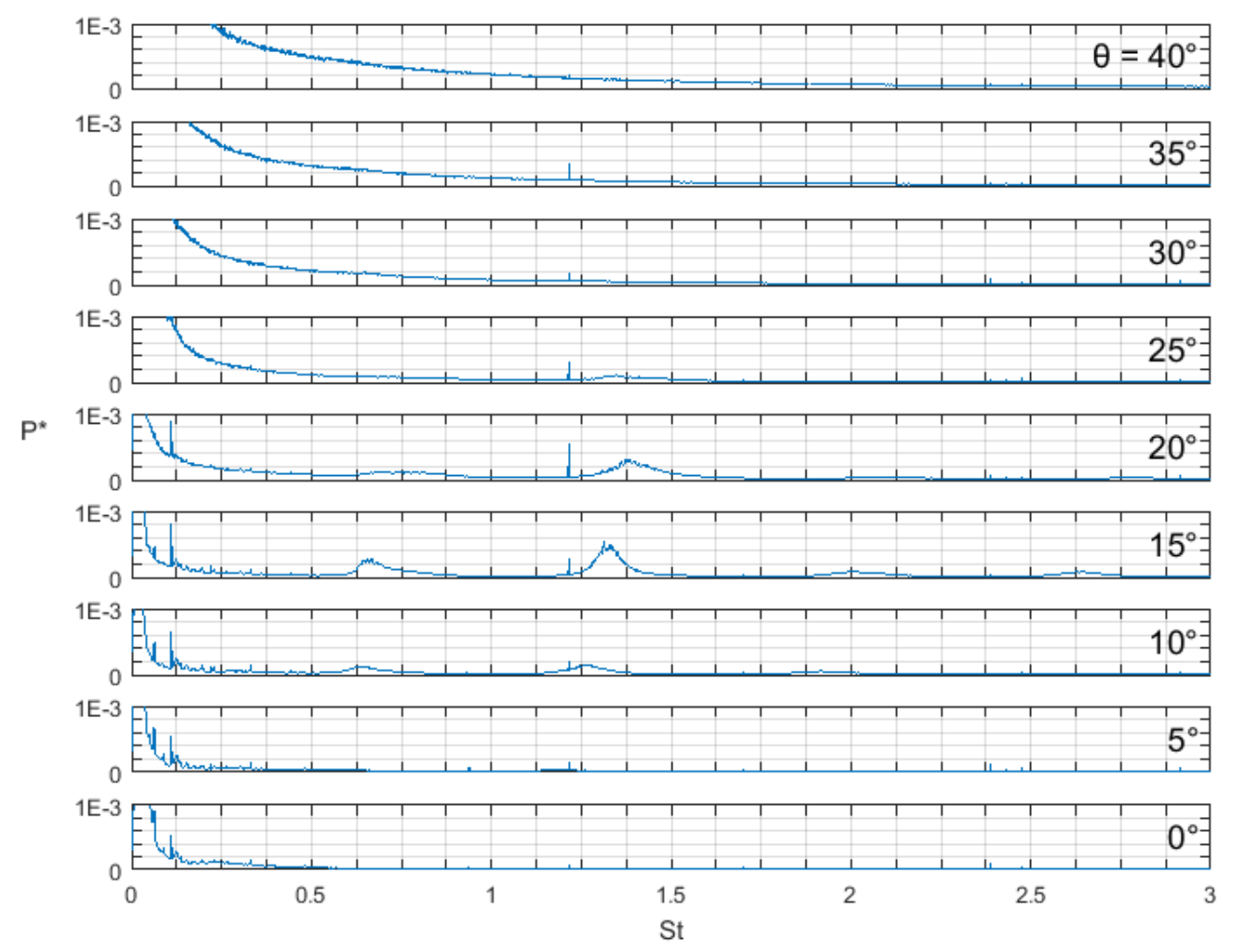

Figure 24: Frequency spectra for a gap width of $12.7 \mathrm{~mm}$, a flow velocity of $20 \mathrm{~m} / \mathrm{s}$, and an angle of incidence of 0 to 40 degrees.

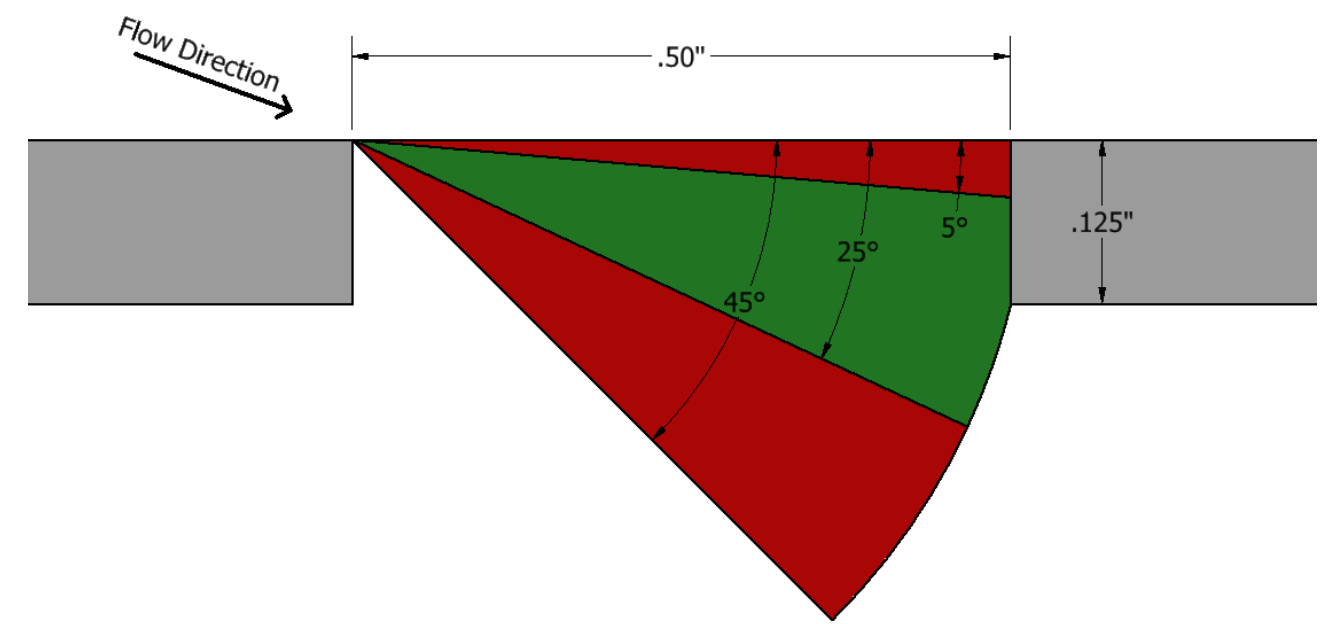

Figure 25: This illustration shows the angles of incidence favorable to the production of tonal noise for a gap width, $H=12.7 \mathrm{~mm}(0.5$ ") and an angle of incidence in the range $0^{\circ} \leq \theta \leq 45^{\circ}$. Green indicates that tones are produced, while red indicates that tones are not produced. Tones are produced for $\theta=5^{\circ}$ to $\theta=25^{\circ}$. 


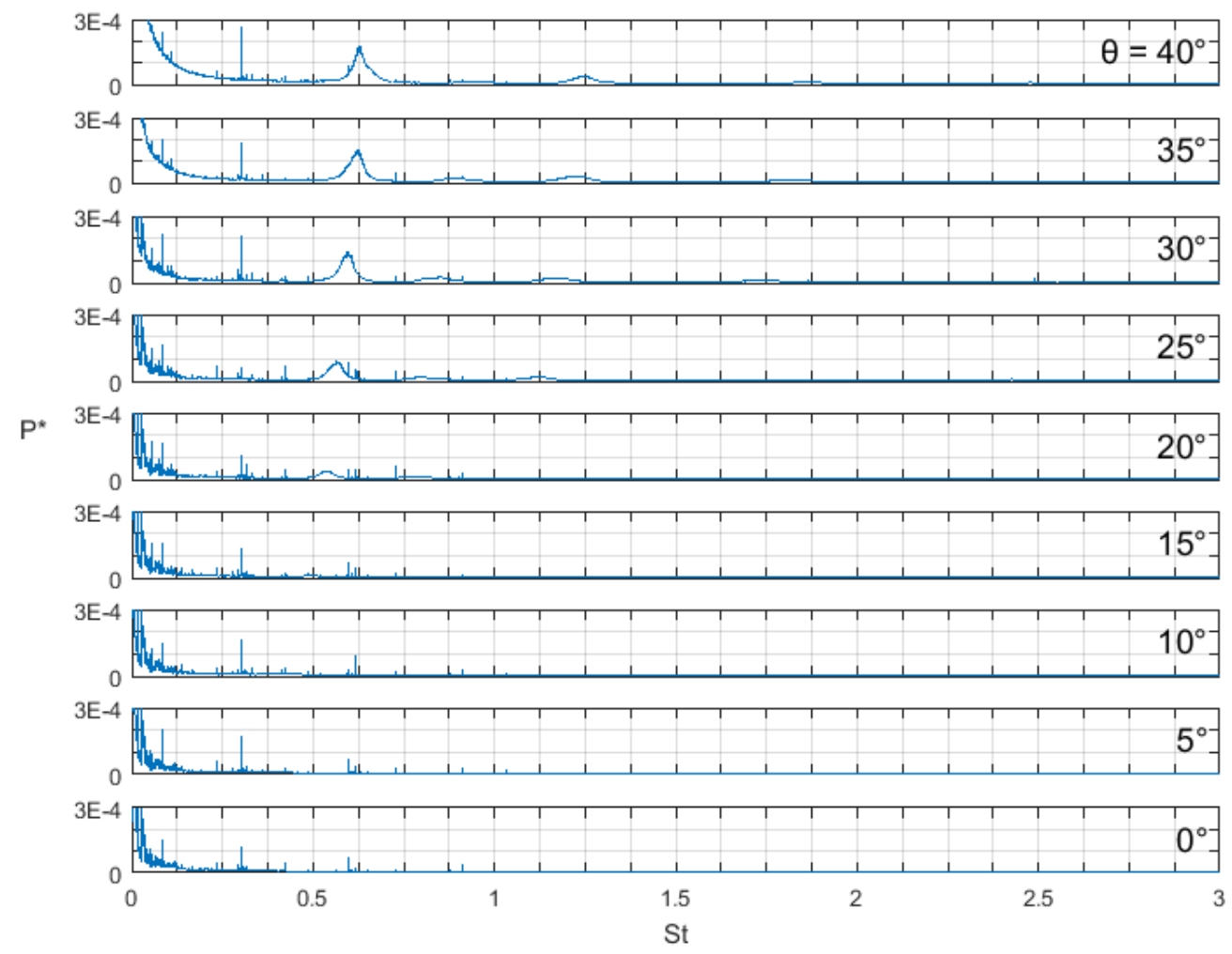

Figure 26: Frequency spectra for a gap width of $3.175 \mathrm{~mm}$, a flow velocity of $20 \mathrm{~m} / \mathrm{s}$, and an angle of incidence of 0 to 40 degrees.

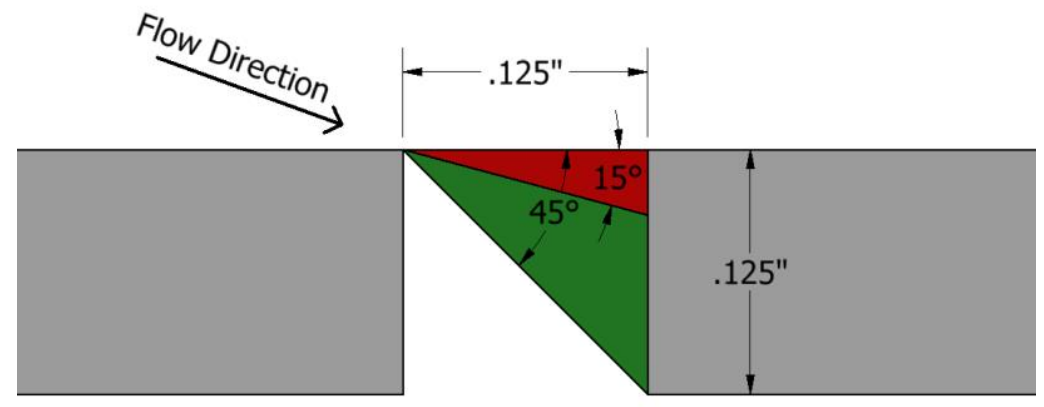

Figure 27: This illustration shows the angles of incidence favorable to the production of tonal noise for a gap width, $H=3.175 \mathrm{~mm}(0.125$ ') and an angle of incidence in the range $0^{\circ} \leq \theta \leq 45^{\circ}$. Green indicates that tones are produced, while red indicates that tones are not produced. Tones are produced for $\theta=15^{\circ}$ to $\theta=45^{\circ}$. 
It is clear from Figure 23, Figure 25, and Figure 27 that tonal noise is produced when the shear layer shed from the trailing edge of the upstream slat impinge on the side of the downstream slat. This impingement is necessary to complete the feedback loop which results in sustained periodic pressure fluctuations. For too small of an angle of incidence, the flow grazes over the surface of the gaps. This is the case with arrow \#1 in Figure 28. For too large of an angle of incidence, the flow passes through the gap and misses the side of the downstream slat, as shown by arrow \#3. On the other hand, arrow \#2 shows the angle of incidence which is favorable for the production of tones. This is confirmed in section 4.4: Particle Image Velocimetry Results, where the results are shown for the three cases of arrows \#1, \#2, and \#3.

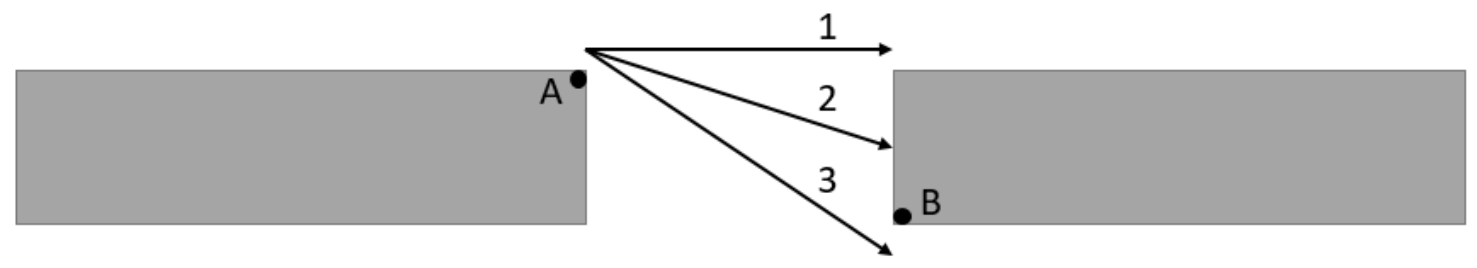

Figure 28: This illustration shows the three classes of inlet flows possible for the slats. In arrow \#1, the angle of incidence is too small to produce tones. In arrow \#3, the angle of incidence is too large to produce tones. In arrow \#2, the flow impinges on the downstream side of the gap and tones may be produced.

In order for the separated shear layer to impinge on the edge of the downstream slat, the angle of incidence at the inlet of the gap must fall within the following range:

$$
0<\theta<\tan ^{-1}\left(\frac{t}{D}\right)
$$

The angle must be greater than 0 degrees, but less than the angle between the top corner of the upstream slat (point $A$ in Figure 28) and the bottom corner of the downstream 
slat (point $B$ in Figure 28). This maximum angle is 14 degrees for a $H / t$ of 4, 27 degrees for a $H / t$ of 2 , and 45 degrees for a $H / t$ of 1 . Therefore, the larger the value of $H / t$, the smaller the angle of incidence required to produce tones and the narrower the range of angles at which tones are produced. The maximum angles which produce tones in Figure 23 and Figure 25 slightly exceed the theoretical limit from equation (14). This is because the angles in Figure 23 and Figure 25 are based on the mean flow from the wind tunnel. In reality, this angle decreases as the flow travels along the length of the plate.

\subsubsection{Effect of flow velocity}

For a given plate geometry, there is a specific range of flow velocity which optimally produces tonal noise. In Figure 29, Figure 30, and Figure 31, the effect of flow velocity is shown for a gap width of $3.175 \mathrm{~mm}, 6.35 \mathrm{~mm}$, and $12.7 \mathrm{~mm}$, respectively. The angle of incidence is set to the optimal angle for each gap width - 30 degrees for a gap width of $3.175 \mathrm{~mm}$, and 15 degrees for a gap width of $6.35 \mathrm{~mm}$ and $12.7 \mathrm{~mm}$.

For the $3.175 \mathrm{~mm}$ gap width $(H / t=1)$, tones occur for velocities of $15 \mathrm{~m} / \mathrm{s}$ and greater $\left(\operatorname{Re}_{D} \geq 3.2 \times 10^{3}\right)$, but are relatively weak in comparison to the other two gap widths. This is shown in Figure 29 below. The largest peak, in terms of normalized pressure $\left(P^{*}\right)$ magnitude occurs for a velocity of $25 \mathrm{~m} / \mathrm{s}$ at a Strouhal number of 0.61 . At a velocity of 15 $\mathrm{m} / \mathrm{s}$ and $20 \mathrm{~m} / \mathrm{s}$, there is a single dominant peak at a Strouhal number of 0.55 to 0.6 with several weaker harmonics. As the velocity is increased to $25 \mathrm{~m} / \mathrm{s}$ and $30 \mathrm{~m} / \mathrm{s}$, the trend changes from one dominant peak to multiple peaks of similar magnitude. 


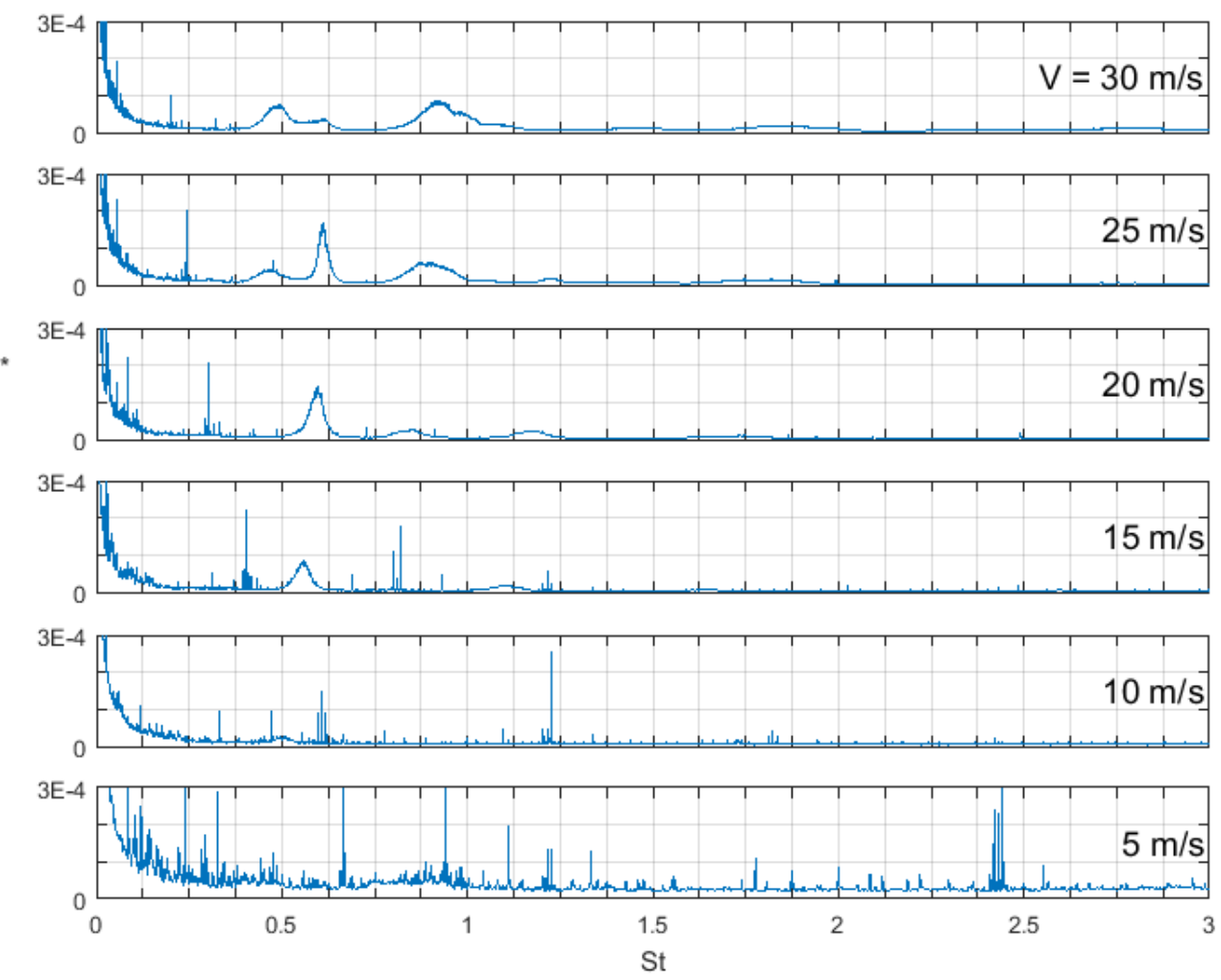

Figure 29: Frequency spectra for a gap width of $3.175 \mathrm{~mm}$, an angle of incidence of 30 degrees, and a flow velocity of 5 to $30 \mathrm{~m} / \mathrm{s}$.

For the $6.35 \mathrm{~mm}$ gap width $(H / t=2)$, tones are observed for velocities of $15 \mathrm{~m} / \mathrm{s}$ and greater $\left(\operatorname{Re}_{D} \geq 6.3 \times 10^{3}\right)$, as shown in Figure 30 . The largest magnitude peak occurs at a velocity of $20 \mathrm{~m} / \mathrm{s}$ and a Strouhal number of 1.15 . There is a single dominant peak until $\mathrm{V}$ $=30 \mathrm{~m} / \mathrm{s}\left(\operatorname{Re}_{D}=12.6 \times 10^{3}\right)$, where a second peak emerges at a Strouhal number of 1.53. This peak is not a harmonic, but instead it is similar to the multiple peaks which were observed at higher velocities for the $H=3.175 \mathrm{~mm}$ case.

In this case, the Strouhal number of the tones increases slightly with increasing flow velocity. This may be a result of the flow being outside the wind tunnel and unbounded. At lower flow velocities, the flow diverges more, decreasing the velocity downstream. This 
lower velocity results in lower frequency tones. This results in lower calculated Strouhal numbers, since the Strouhal number is based on the wind tunnel exit velocity, not the local velocity at the microphone location.

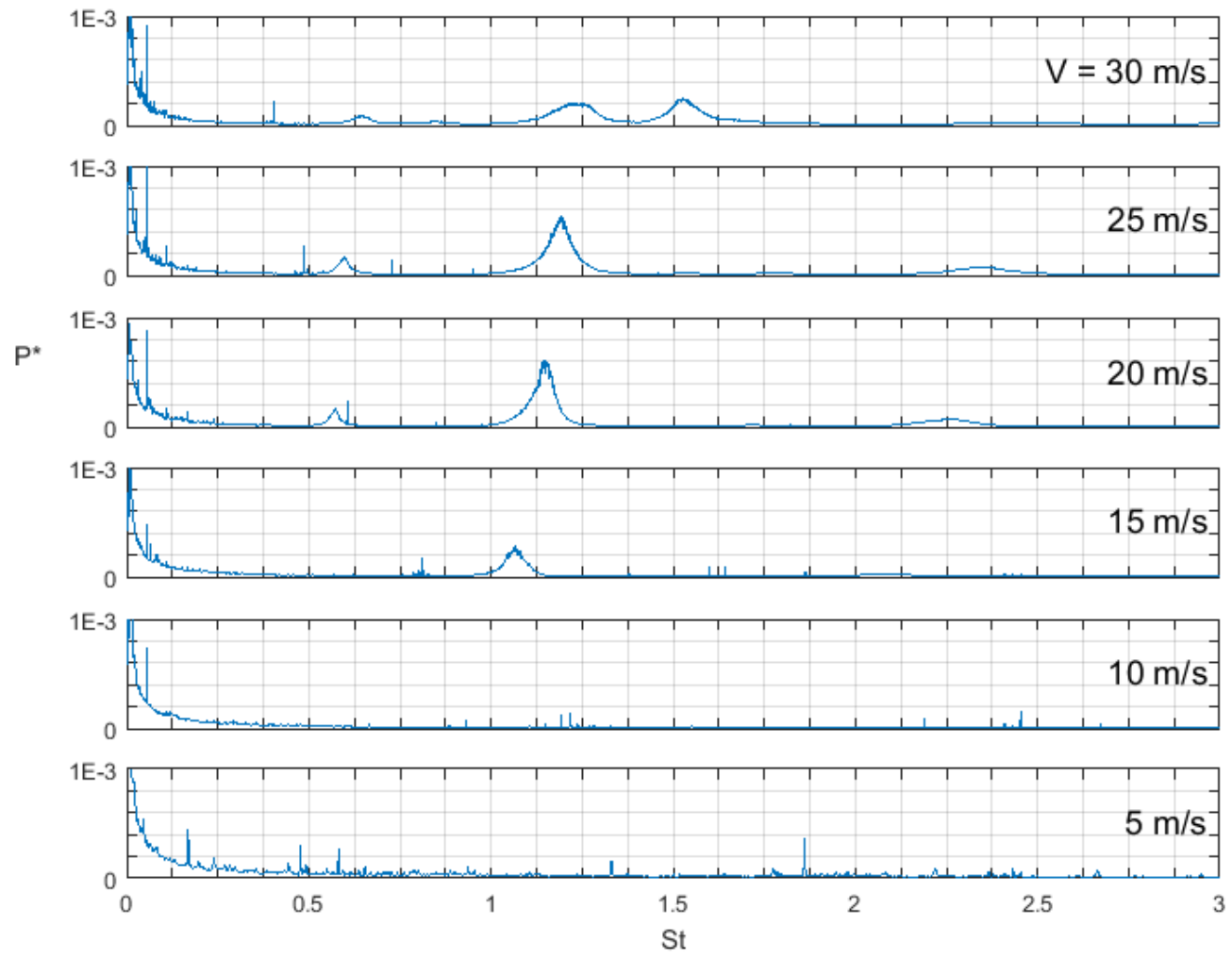

Figure 30: Frequency spectra for a gap width of $6.35 \mathrm{~mm}$, an angle of incidence of 15 degrees, and a flow velocity of 5 to $30 \mathrm{~m} / \mathrm{s}$.

For the $12.7 \mathrm{~mm}$ gap width $(H / \mathrm{t}=4)$, tones occur for velocities of $10 \mathrm{~m} / \mathrm{s}$ and greater $\left(R e_{D} \geq 8.4 \times 10^{3}\right)$, as shown in Figure 31 . For this case, the largest magnitude tone is observed at a flow velocity of $10 \mathrm{~m} / \mathrm{s}$, when expressed in terms of normalized pressure, $P^{*}$. The largest peak is at a Strouhal number of 1.31, with a slightly weaker subharmonic at a Strouhal number of 0.65 . The magnitude of the peaks decreases with increasing flow 
velocity beyond $10 \mathrm{~m} / \mathrm{s}$. For this case, there are multiple harmonics and sub-harmonics for all flow velocities.
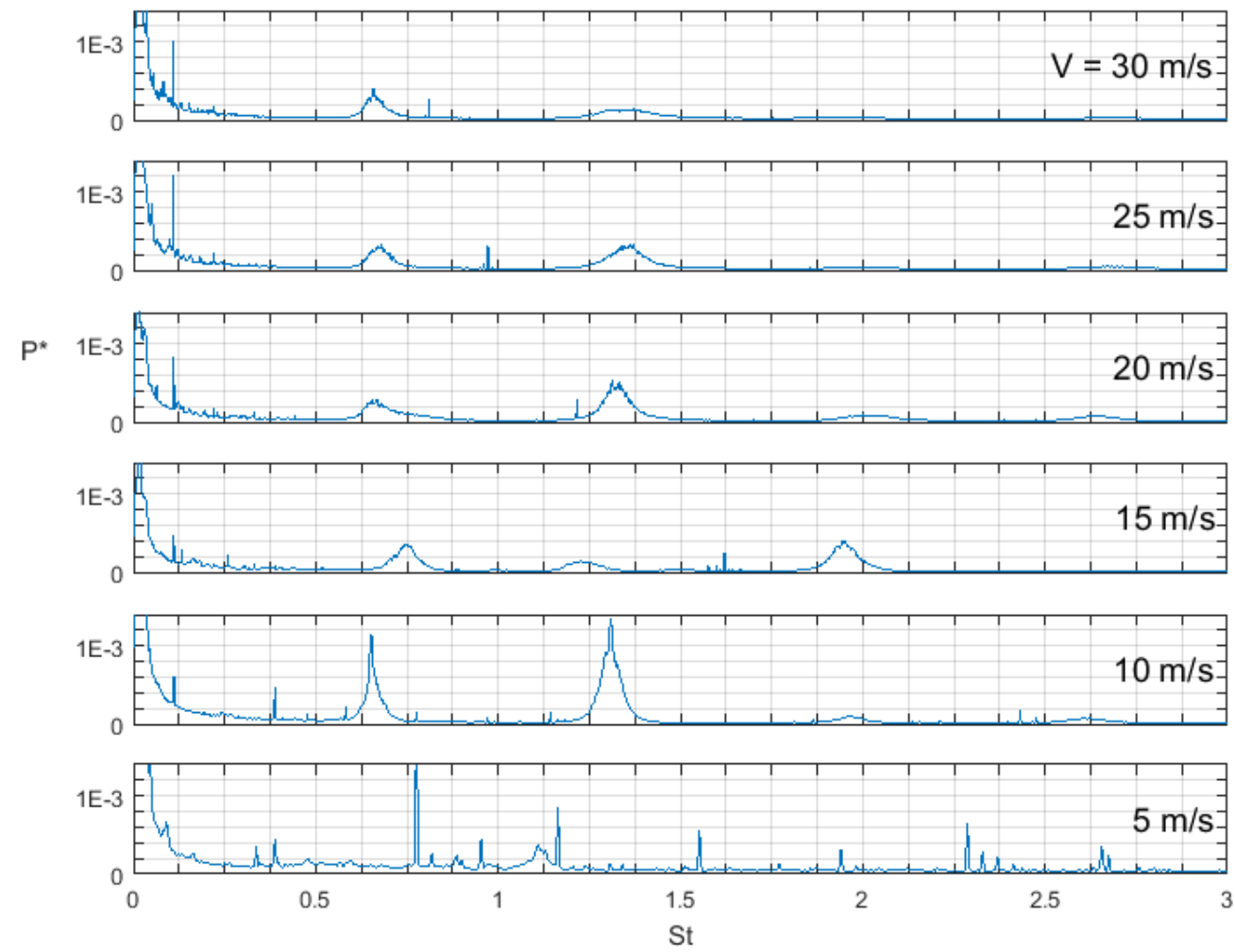

Figure 31: Frequency spectra for a gap width of $12.7 \mathrm{~mm}$, an angle of incidence of 15 degrees, and a flow velocity of 5 to $30 \mathrm{~m} / \mathrm{s}$.

In general, there is an ideal flow velocity at which $P^{*}$ of the dominant peak is at a maximum. Above and below this optimal velocity, the magnitude of the peaks decrease. This optimal velocity is lower for larger gap widths.

For complete results at an angle of incidence of 0 to 40 degrees, see Appendix B.2.1: Effect of flow velocity, gap width $=3.175 \mathrm{~mm}$, Appendix B.2.2: Effect of flow velocity, gap width $=6.35 \mathrm{~mm}$, and Appendix B.2.3: Effect of flow velocity, gap width $=12.7 \mathrm{~mm}$. 


\subsubsection{Effect of gap width}

As shown in the previous two subsections, the gap width has a significant effect on the velocity and angle of incidence at which tonal noise is produced. For the same slat thickness, a larger gap width produces tones at lower flow velocities and smaller angles of incidence. At a larger gap width, the Strouhal number of the measured tonal noise is greater. This is shown in Figure 32 for a velocity of $20 \mathrm{~m} / \mathrm{s}$ and an angle of incidence of 0 to 40 degrees. For the $6.35 \mathrm{~mm}$ gap width, tones with a Strouhal number of 1.1 to 1.2 are seen from $\theta=10$ to 20 degrees. For a $12.7 \mathrm{~mm}$ gap width, tones with a similar magnitude are seen at the same angles with a Strouhal number of 1.3 to 1.4 . Finally, for a $3.175 \mathrm{~mm}$ gap width, relatively weak tones with a Strouhal number around 0.6 are seen at $\theta=30$ degrees and greater.

For architectural applications, the velocity and angle of the wind are functions of the climate and weather, and cannot be controlled by designers. The geometry of the plate is the one variable which can be controlled. Larger gap widths produce tones at lower flow velocities. However, the magnitude of these tones is lower, when expressed as actual, nonnormalized sound pressure. Therefore, if the range of wind velocities which a plate is expected to be exposed to is known, one of two approaches can be taken when choosing a hole diameter. First, a small hole size can be chosen, such that the wind rarely, if ever, reaches a high enough velocity to produce tones. The downside of this approach is that if the wind does reach a sufficiently high velocity, the magnitude of the tones will be great. The second approach is to use a larger hole size, such that tones are produced at low wind velocities, but these tones are sufficiently weak in magnitude to not be an irritant. 


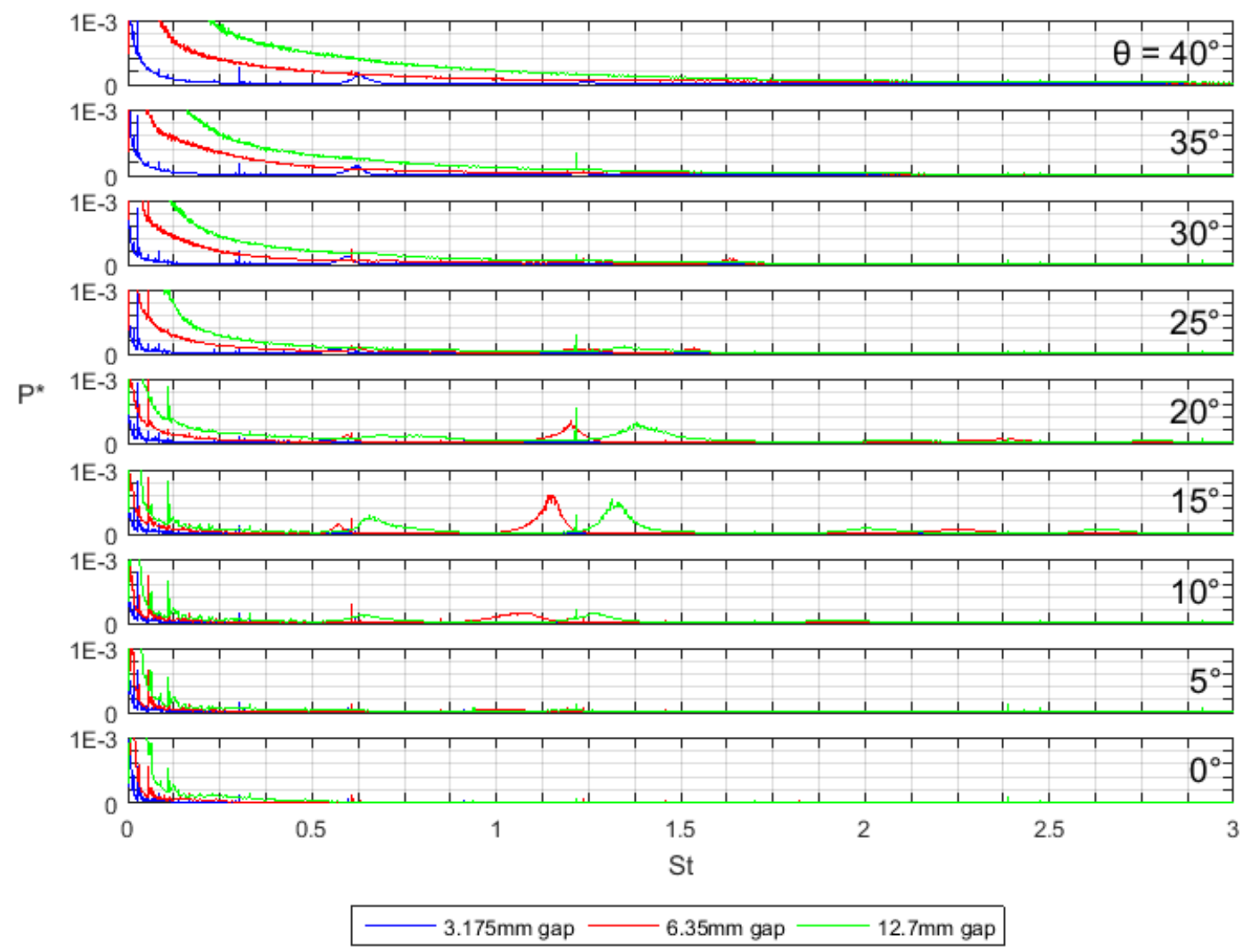

Figure 32: Frequency spectra for a gap width of $3.175 \mathrm{~mm}\left(1 / 8^{\prime \prime}\right), 6.35 \mathrm{~mm}\left(1 / \mathrm{4}^{\prime \prime}\right)$, and $12.7 \mathrm{~mm}\left(1 / 2^{\prime \prime}\right)$, a flow velocity of $20 \mathrm{~m} / \mathrm{s}$, and an angle of incidence of 0 to 40 degrees.

\subsubsection{Effect of streamwise position}

In order to identify the source of the tonal noise and understand the mechanism that produces it, microphone measurements are taken along the length of the plate in the streamwise direction. Using the coordinate system defined in Figure 13, the $Y$-position of the microphone is kept fixed at $Y=-25.4 \mathrm{~mm}$. The $X$-position is varied along the length of the plate in the streamwise direction. Measurements are taken at every $3^{\text {rd }}$ gap location, with the exception of case $\# 2$, where the measurements are taken at every gap location, and 
case \#4, where measurements are only taken from the $3^{\text {rd }}$ to $24^{\text {th }}$ gap locations. For a gap width of $6.35 \mathrm{~mm}(1 / 4$ inches $)$ and slats with a width of $23.29 \mathrm{~mm}(0.917$ inches $)$, every $3^{\text {rd }}$ gap corresponds to a distance of $88.9 \mathrm{~mm}$ (3.5 inches) between measurements. Under this configuration, there are a total of 30 slats and 29 gaps supported by the $914.4 \mathrm{~mm}$ (36 inches) long frame. The measurements were taken four times for a gap width of $6.35 \mathrm{~mm}$ and an angle of incidence of 15 degrees, corresponding to the four cases in Figure 33 and Figure 34. Between each set of measurements, the apparatus was disassembled and reassembled, including removing and reattaching the slats. This was done to confirm the repeatability of the measurements. The resulting frequency spectra are shown in Figure 33. Case \#3 and case \#4 in Figure 33 are measured with the surface of the slats covered in thin vinyl tape. This was done in order to minimize reflections when taking PIV images, as discussed in section 3.3: Particle Image Velocimetry Measurements.

The results of Figure 33 show a tonal peak with a Strouhal number of 1.1 to 1.2 at all gap locations. The magnitude of this peak increases until around the $15^{\text {th }}$ gap, then remains relatively constant until the $27^{\text {th }}$ gap. After the last gap, at the downstream end of the entire plate, the magnitude drops off quickly. This is shown in an alternate way in Figure 34. Here, the overall sound pressure level (OSPL) of the peaks is calculated and plotted in units of decibels as a function of the $X$ position. The OSPL of the peaks is calculated over a frequency range of $4.7 \mathrm{kHz}$, centered around the frequency of the tone. At the bottom of Figure 34, a cross-sectional view of the slats is overlaid to show the position of the measurements relative to the slats. At the top, the locations of the $6^{\text {th }}, 12^{\text {th }}, 18^{\text {th }}$, and $24^{\text {th }}$ gaps are identified. This figure clearly shows an increase in the OSPL as the flow develops 
along the plate, followed by a region of relatively constant OSPL, followed by a quick decrease at the downstream end of the plate.

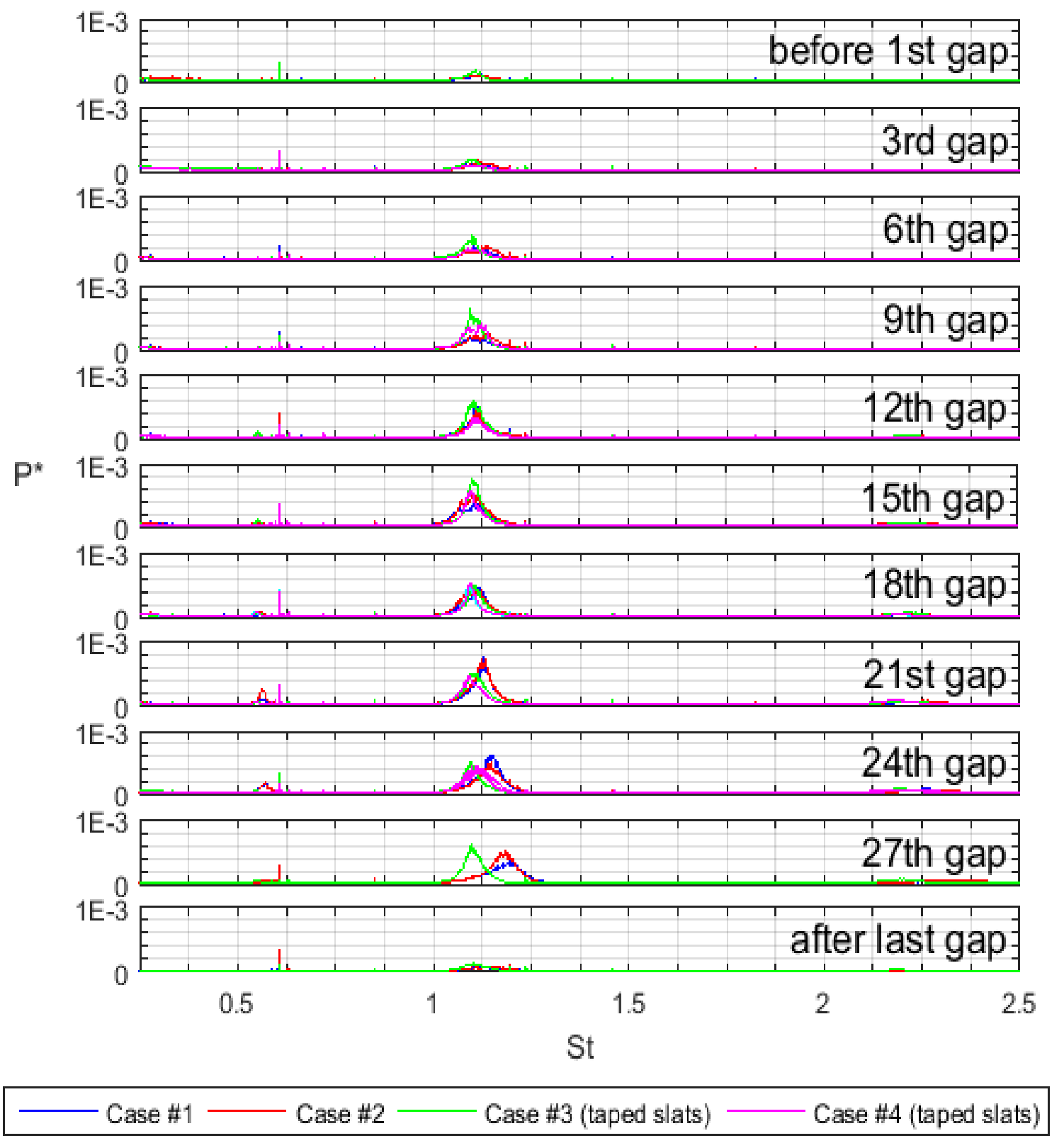

Figure 33: Frequency spectra for a gap width of $6.35 \mathrm{~mm}$, an angle of incidence of $15^{\circ}$, and a flow velocity of $20 \mathrm{~m} / \mathrm{s}$. Results are shown for un-taped (blue \& red) and taped (green \& pink) slats. The streamwise position is varied, from before the first gap to after the last gap. For Case \#4, spectra are only shown for the $3^{\text {rd }}$ to $24^{\text {th }}$ gaps. 


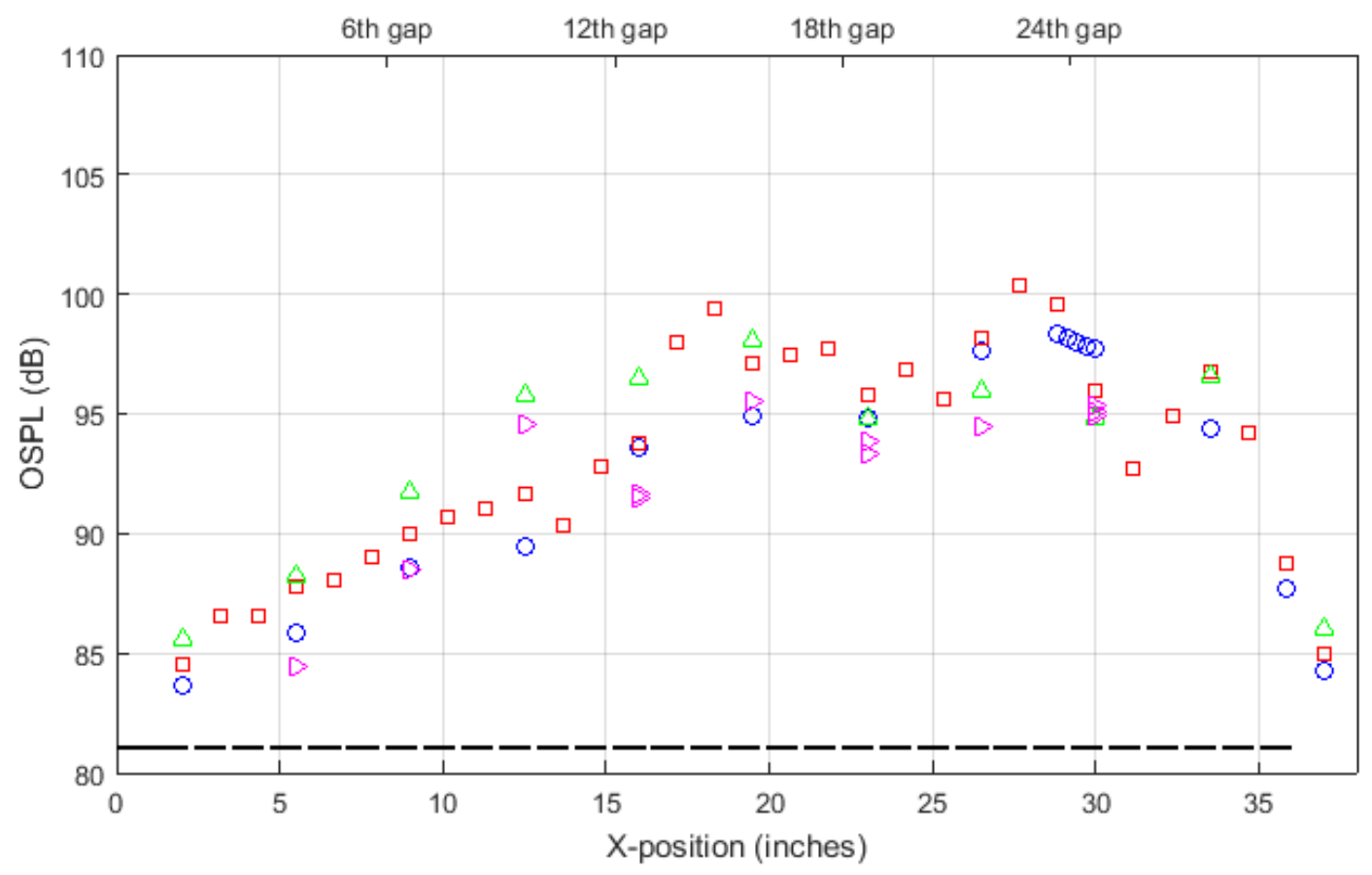

$0 \quad$ Case \#1 $\quad \square \quad$ Case \#2 $\triangle \quad$ Case \#3 (taped slats) $\quad>\quad$ Case \#4 (taped slats)

Figure 34: Overall sound pressure level for a gap width of $6.35 \mathrm{~mm}$, an angle of incidence of $15^{\circ}$, and a flow velocity of $20 \mathrm{~m} / \mathrm{s}$. Results are shown for un-taped (blue $\&$ red) and taped (green \& pink) slats. The streamwise position is varied, from before the first gap to after the last gap.

There is some scatter in the data in Figure 33 and Figure 34, due to a variety of sources of error. The geometric parameters of the plate change slightly when the assembly is disassembled and reassembled. This includes the order of the slats, the gap width between each slat and the angle of incidence of the plate. The slats may become somewhat twisted or offset during reassembly, as was discussed in section 3.4: Geometric Inconsistencies. The position of the microphone can also change slightly from one set of measurements to the next. Despite this scatter, the main trend remains clear for all four cases. 
Figure 33 and Figure 34 show that taping the slats has a minor effect on the acoustic results. The general trend for the un-taped and taped slats is similar, with some small differences. The differences can be attributed to experimental uncertainty and repeatability. In some cases, the Strouhal number of the tones increases slightly at the downstream end of the plate, from around the $21^{\text {st }}$ gap to the $27^{\text {th }}$ gap. This is likely due to a slight increase in the local flow velocity at the downstream end of the plate.

A similar trend is seen for a mean flow velocity of $15 \mathrm{~m} / \mathrm{s}, 25 \mathrm{~m} / \mathrm{s}$, and $30 \mathrm{~m} / \mathrm{s}$. For these results, see Appendix B.2.4: Effect of streamwise position. 


\subsection{Particle Image Velocimetry Results}

\subsubsection{Laser positioned in front of slats}

PIV images were taken with the laser positioned on both the front side and the back side of the slats. With the laser at the front side of the slats (positive $Y$-direction in Figure 13), the flow approaching the gaps can be seen, as well as the flow between the slats. With the laser at the back side of the slats (negative $Y$-direction in Figure 13), the flow between the slats can still be seen, as well as the flow leaving the gaps. The two perspectives combine to give a complete picture of the flow field in the area around the slats.

The results for the front side of the slats are shown in Figure 35 and Figure 36 for the case of $H=6.35 \mathrm{~mm}, \theta=15^{\circ}$, and $V=20 \mathrm{~m} / \mathrm{s}$. This is the case which was found to have the most well-defined single peaks in the acoustic results (Figure 22). The mean flow travels from left to right in these images. The $X-Y$ axis corresponds with that previously defined in Figure 13, but the origin is defined as the leading edge of the gap. The grey rectangles in these figures are superimposed onto the images to show the location of the slats. These images are focused on the $24^{\text {th }}$ downstream gap. In the acoustic results, this was found to be the location where the flow is fully developed and the magnitude of the tonal peak is at its maximum (Figure 34).

Figure 35 shows the phase-locked velocity magnitude, which includes the $X$ and $Y$ components of the velocity field. It can be seen that the flow accelerates at the leading edge of the gap. Similarly, there is a region of lower velocity flow at the trailing edge of the gap. There is a steep velocity gradient originating at the leading edge of the gap, leading to the formation of a free shear layer. This gradient occurs between the mean flow, which is 
approximately $20 \mathrm{~m} / \mathrm{s}$, and the nearly stagnant flow between the slats. Due to the angle of incidence of the mean flow relative to the slats, the flow impinges on the side of the downstream slat, and is directed to exit the backside of the gap. The phase-locked velocity field reveals that the flow exiting the gap periodically alternates between higher and lower velocity. One of these high velocity pulses exits the gap for each period of oscillation.

The phase-locked vorticity field is calculated from the velocity field data and plotted in Figure 36. Contours of the $d_{2}$ parameter are overlaid onto the images to show the locations of the individual vortices. A region of high vorticity forms across the free shear layer originating at the leading edge of the gap. This vorticity is highly negative, which corresponds to clockwise rotation in the images. Individual coherent vortices form in the shear layer and impinge on the side of the downstream slat. They exit the gap in a direction perpendicular to the slats. These vortices remain coherent as they exit the backside of the gap and move away from the slats. 

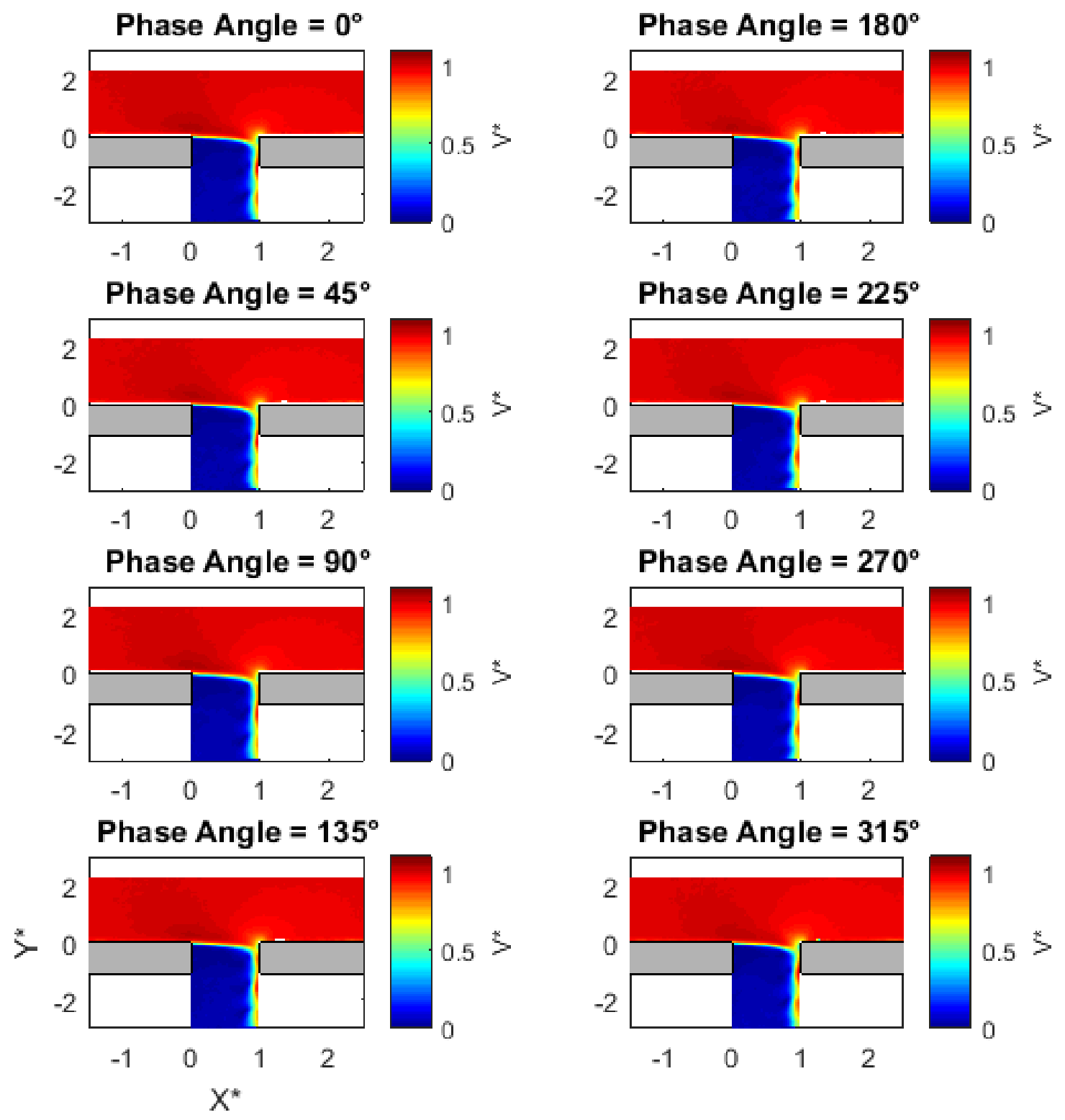

Figure 35: PIV results showing the phase-locked velocity field at the 24th gap for a gap width of $6.35 \mathrm{~mm}$, an angle of incidence of 15 degrees, and a flow velocity of 20 $\mathrm{m} / \mathrm{s}$. Images are taken with the laser on the front side of the slats. 

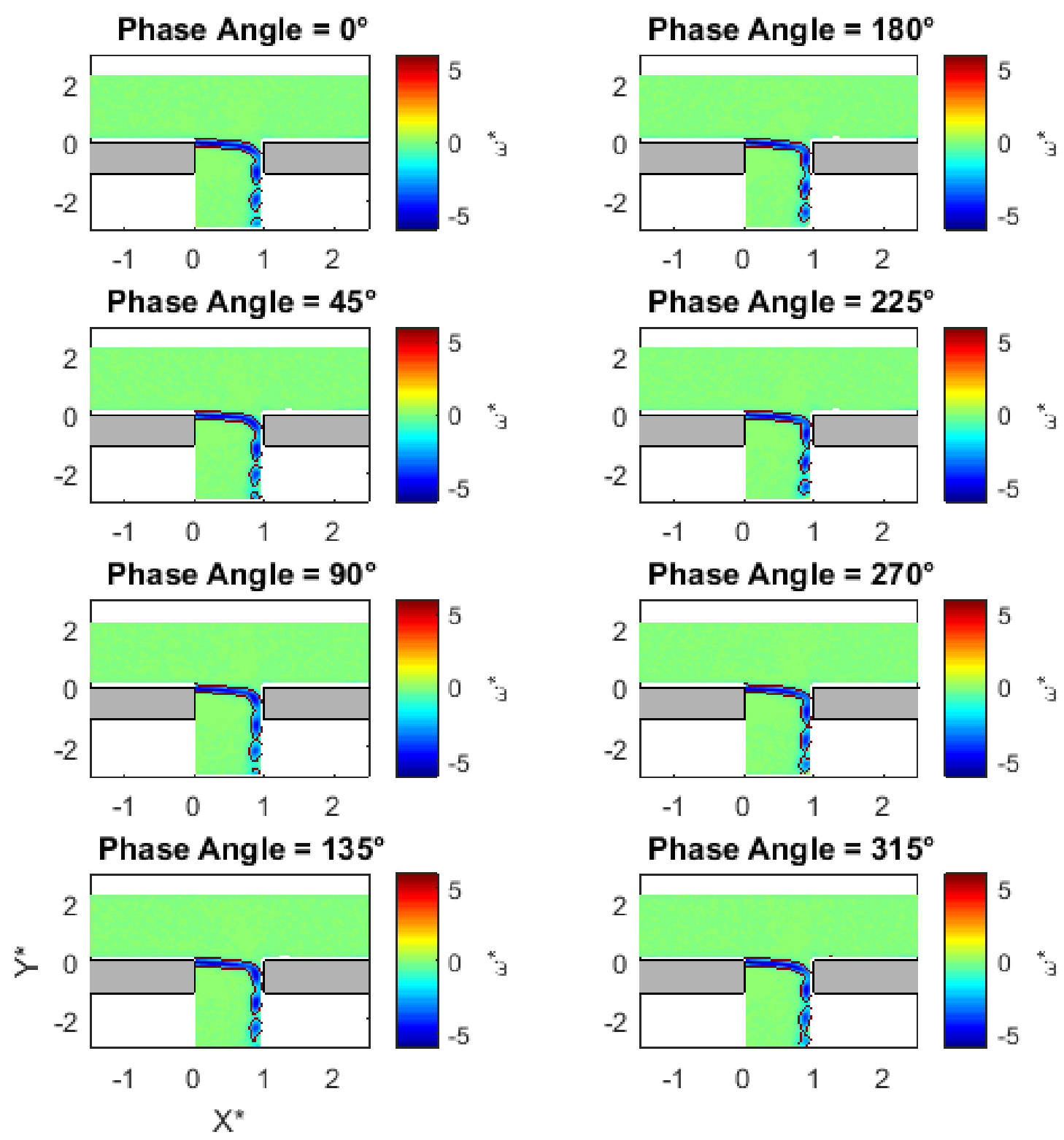

Figure 36: PIV results showing the phase-locked vorticity field at the 24th gap for a gap width of $6.35 \mathrm{~mm}$, an angle of incidence of 15 degrees, and a flow velocity of 20 $\mathrm{m} / \mathrm{s}$. Images are taken with the laser on the front side of the slats. 


\subsubsection{Laser positioned behind slats}

Because the laser sheet is blocked by the slats, the flow field behind the slats is hidden from view in Figure 35 and Figure 36. In order to investigate this region, the laser is positioned on the other side of the slats. From this point of view, the flow field in the gap and behind the slats can be seen. It is found that this perspective gives a more complete picture of the sound generation mechanism, and is therefore used for the remainder of the images, at a variety of angles of incidence, flow velocities, and gap locations. The gap width and the slat dimensions are kept constant for all cases.

Figure 37 and Figure 38 show velocity magnitude and vorticity fields for the same case as Figure 35 and Figure 36, except with the laser positioned behind the slats. From this point of view, it can be seen that there are pairs of vortices, rather than individual vortices as indicated previously in Figure 36. A region of high vorticity forms across the free shear layer originating at the leading edge of the gap. Clockwise rotating vortices form in this shear layer and impinge on the downstream slat. At the point where the flow exits the gap, it separates from the backside edge of the slats and positive vorticity, counterclockwise rotating vortices are shed. These vortices, together with the shear vortices, form vortex pairs, where one half of the pair has a positive vorticity and the other half has a negative vorticity. The frequency of these vortex pairs is equal to the frequency of the dominant tonal peak found in the acoustic measurements. Therefore, the periodic shedding of these vortex pairs is the source of this tonal noise. 

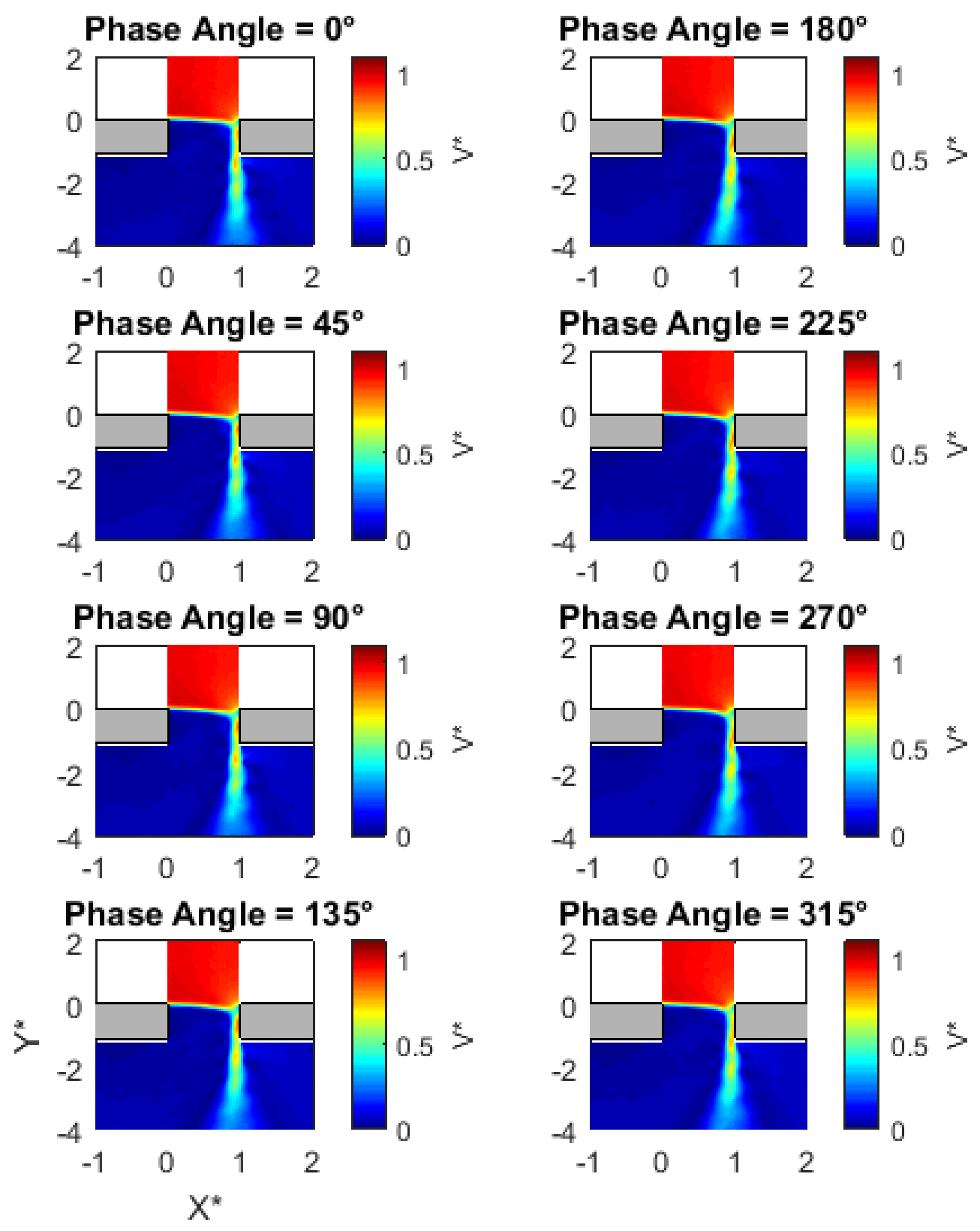

Figure 37: PIV results showing the phase-locked velocity field at the 24th gap for a gap width of $6.35 \mathrm{~mm}$, an angle of incidence of 15 degrees, and a flow velocity of 20 $\mathrm{m} / \mathrm{s}$. Images are taken with the laser on the back side of the slats. 

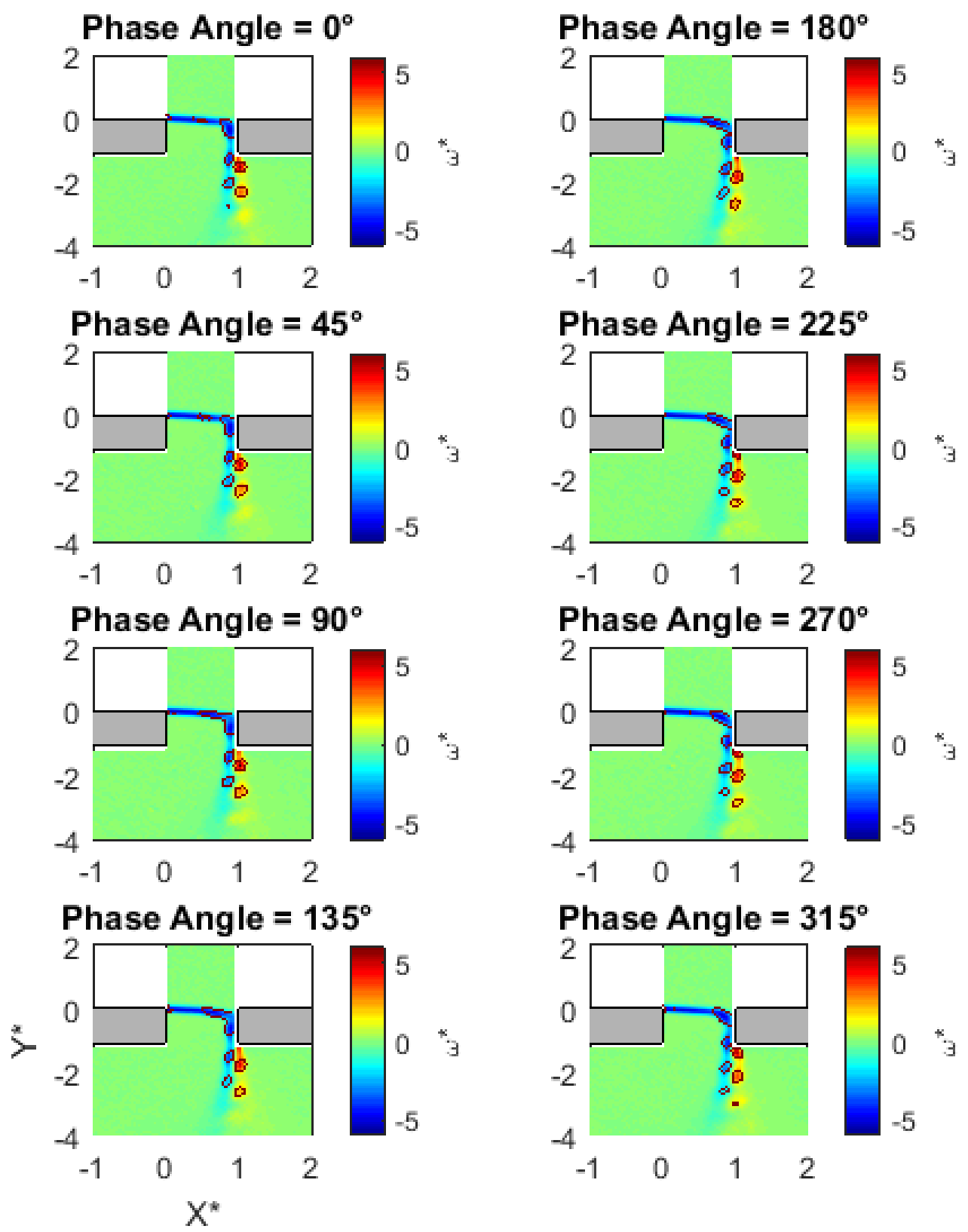

Figure 38: PIV results showing the phase-locked vorticity field at the 24th gap for a gap width of $6.35 \mathrm{~mm}$, an angle of incidence of 15 degrees, and a flow velocity of 20 $m / s$. Images are taken with the laser on the back side of the slats. 


\subsubsection{Effect of angle of incidence}

The acoustic results showed that tonal noise is produced only for a specific range of angles. For the case of a gap width of $6.35 \mathrm{~mm}$ and a mean velocity of $20 \mathrm{~m} / \mathrm{s}$, this range of angles was approximately 5 to 30 degrees, with the most pronounced tones at 10 to 30 degrees (see Figure 22). For angles of 0 degrees or angles of 35 degrees and larger, only broadband noise is produced.

PIV results are shown below for the case at which the angle is too small to produce tones and the case at which the angle is too large to produce tones. Figure 39 and Figure 40 show the velocity and vorticity fields for a gap width of $6.35 \mathrm{~mm}$ at an angle of incidence of 0 degrees. Figure 41 and Figure 42 show the same for an angle of incidence of 35 degrees. For both angles, only the $V=20 \mathrm{~m} / \mathrm{s}$ case is shown. However, a velocity of 15 to $30 \mathrm{~m} / \mathrm{s}$ produces similar results, when expressed in non-dimensional terms. For results at other velocities, see Appendix B.3.1: Effect of angle of incidence. Because no tonal noise is produced in these cases, there is no periodic signal to phase-lock to, so phase-locked images could not be produced. The results shown are the average flow field. 


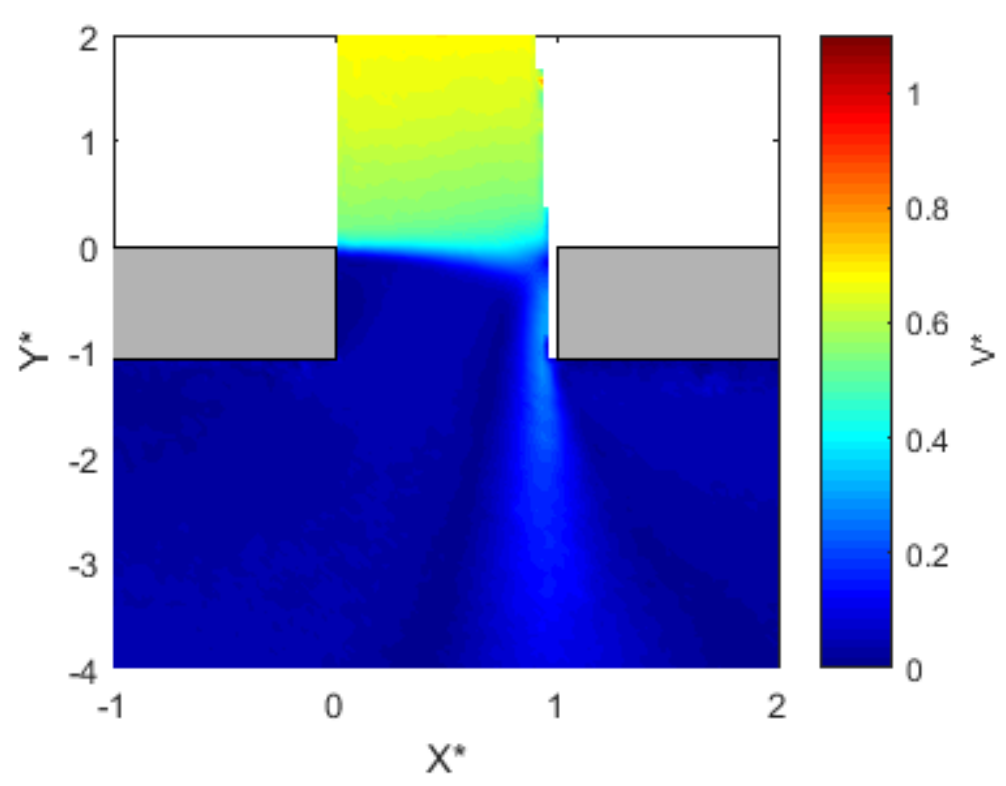

Figure 39: PIV results showing the average velocity field at the 24 th gap for a gap width of $6.35 \mathrm{~mm}$, an angle of incidence of 0 degrees, and a flow velocity of $20 \mathrm{~m} / \mathrm{s}$.

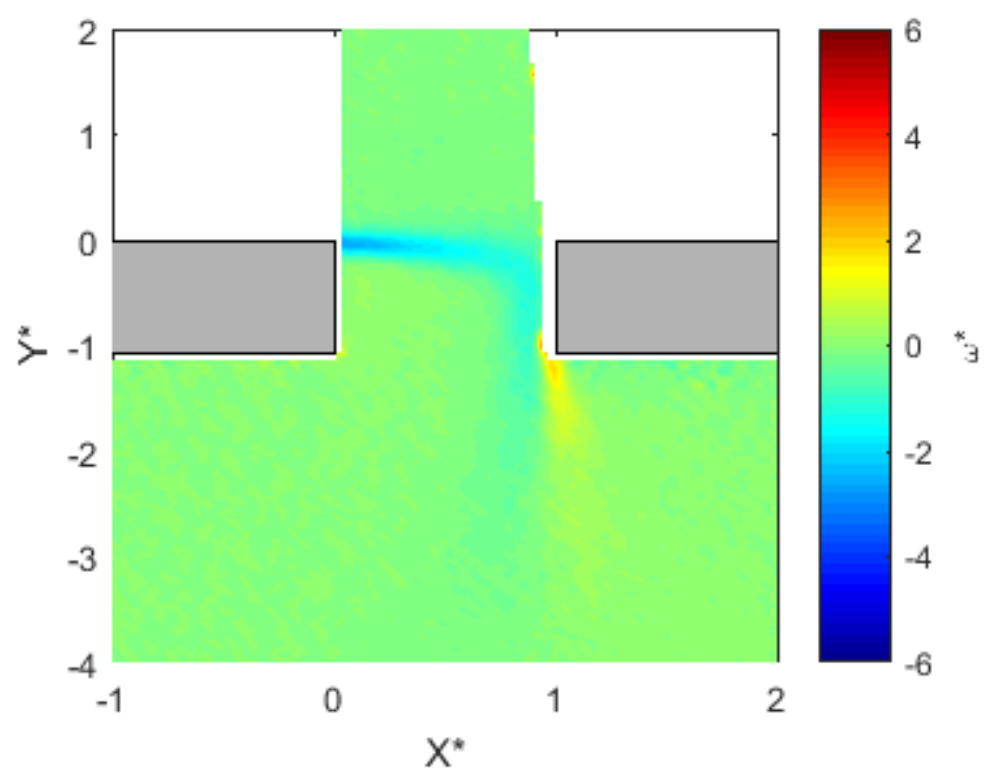

Figure 40: PIV results showing the average vorticity field at the 24th gap for a gap width of $6.35 \mathrm{~mm}$, an angle of incidence of 0 degrees, and a flow velocity of $20 \mathrm{~m} / \mathrm{s}$. 


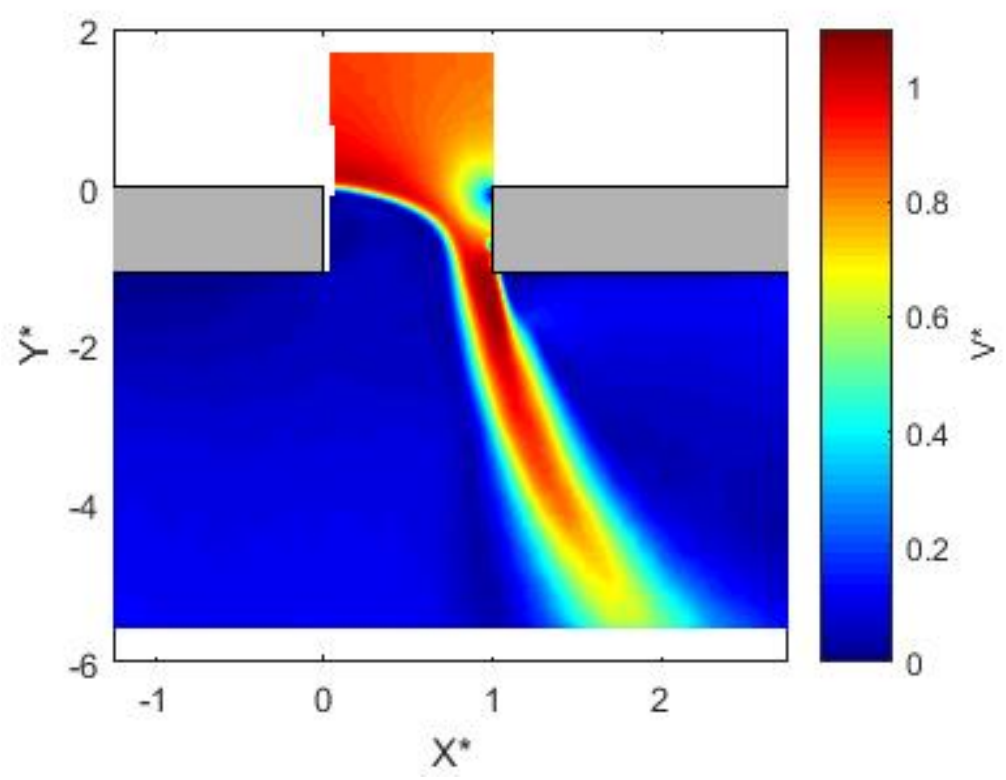

Figure 41: PIV results showing the average velocity field at the 24th gap for a gap width of $6.35 \mathrm{~mm}$, an angle of incidence of 35 degrees, and a flow velocity of $20 \mathrm{~m} / \mathrm{s}$.

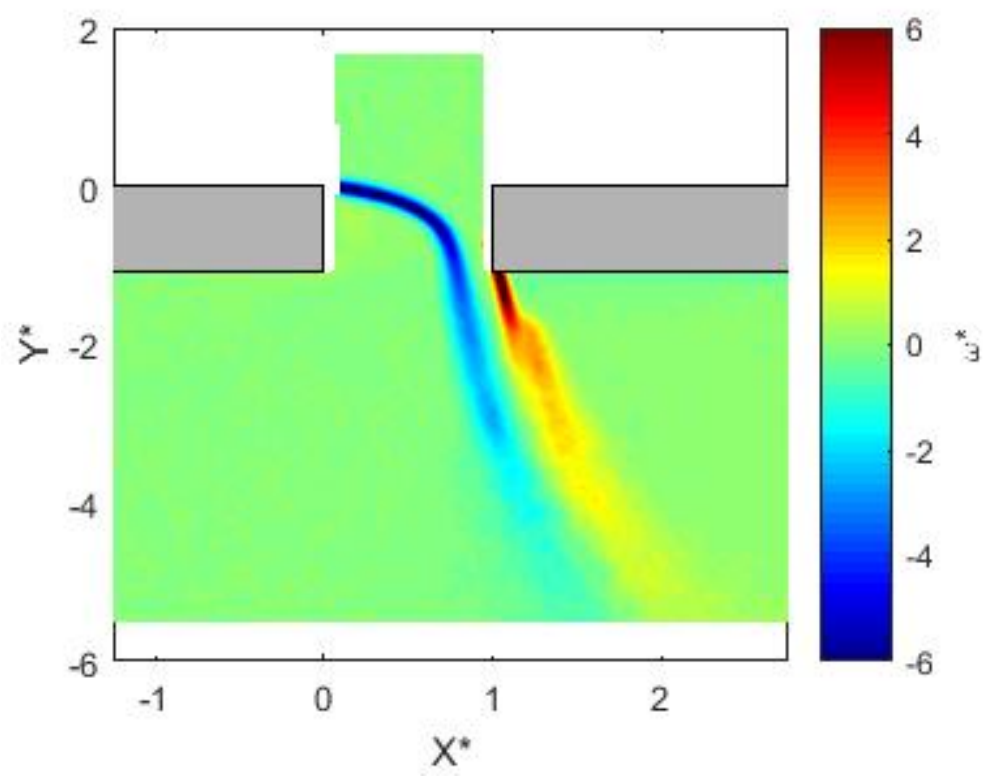

Figure 42: PIV results showing the average vorticity field at the 24th gap for a gap width of $6.35 \mathrm{~mm}$, an angle of incidence of 35 degrees, and a flow velocity of $20 \mathrm{~m} / \mathrm{s}$. 
In Figure 43, the average velocity and vorticity at an angle of incidence of $0^{\circ}, 15^{\circ}$, and $35^{\circ}$ are compared. For the case of $\theta=0^{\circ}$, the flow grazes over the surface of the gap and very little flow passes through the gap. The vorticity in the gap is very weak in comparison to the $15^{\circ}$ and $35^{\circ}$ cases and dissipates quickly. As the angle is increased, a greater amount of flow passes through the gap, impinging on the edge of the downstream slat. This is the case for $\theta=15^{\circ}$, where the shear layer and its vortices impinge on the downstream slat at around $Y^{*}=-0.25$. It then moves vertically along the side of the downstream slat until it leaves the gap at the downstream corner. As the angle of incidence is increased further, the point where the shear layer impinges moves lower. Around $\theta=35^{\circ}$, the shear layer totally misses the bottom corner of the downstream slat. This stops the feedback loop, since the shear layer no longer impinges on the downstream edge. In this case, the gap acts as a nozzle, accelerating the flow to a velocity higher than the mean flow velocity, but not producing any periodic feedback. 


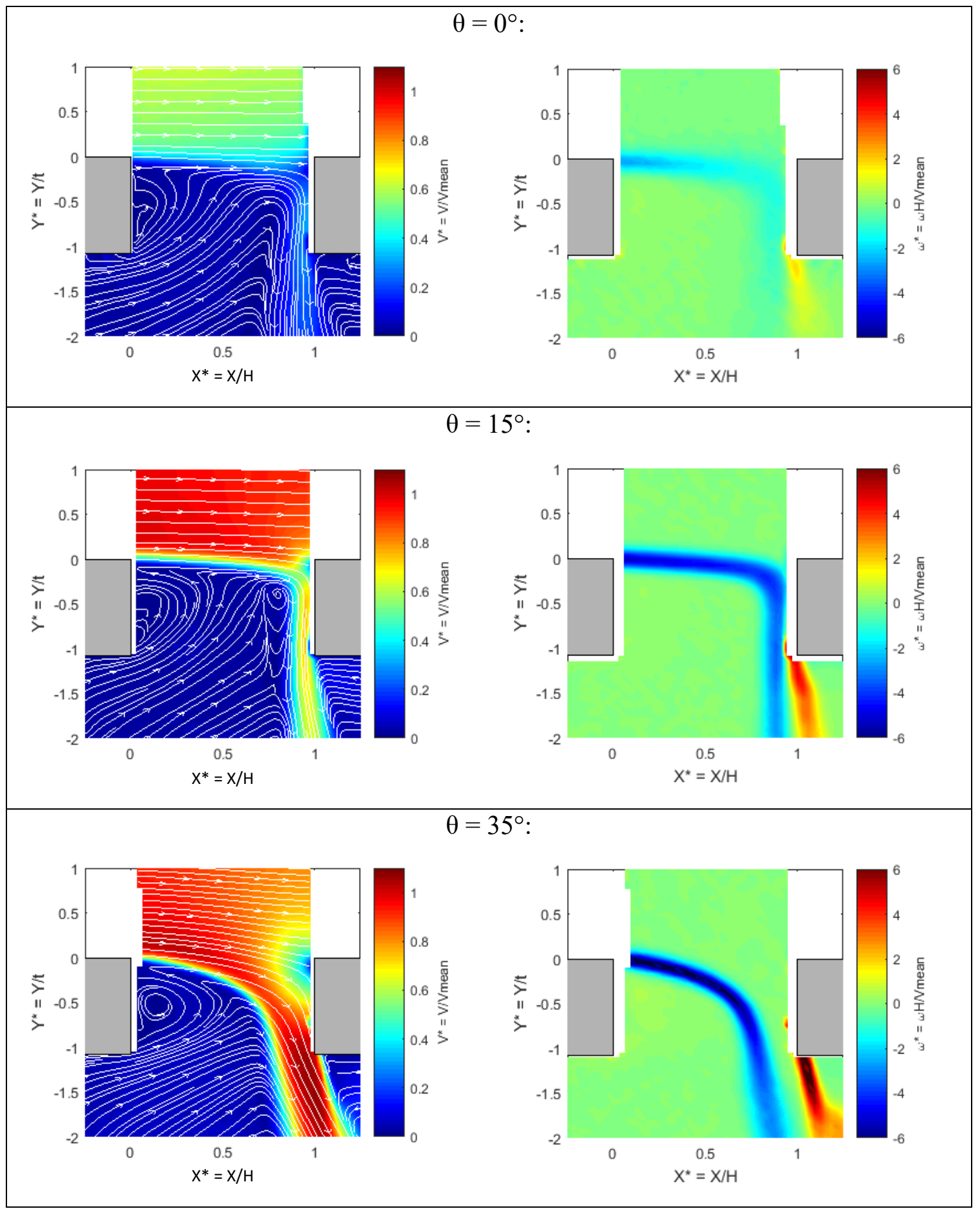

Figure 43: PIV results showing the average velocity (left) and vorticity (right) fields at the 24th gap for a gap width of $6.35 \mathrm{~mm}$ and a flow velocity of $20 \mathrm{~m} / \mathrm{s}$. The angle of incidence is $0^{\circ}$ for the top row, $15^{\circ}$ for the middle row, and $35^{\circ}$ for the bottom row.

The white lines are streamlines. 


\subsubsection{Effect of flow velocity}

In the acoustic results, it was found that tones are produced for a specific range of flow velocities. For the case of a gap width of $6.35 \mathrm{~mm}$ and an angle of incidence of 15 degrees, this range of velocity was approximately 15 to $30 \mathrm{~m} / \mathrm{s}$, with the largest tonal peaks at 20 to $25 \mathrm{~m} / \mathrm{s}$ (see Figure 30). Focusing on the $24^{\text {th }}$ gap location, for a gap width of 6.35 $\mathrm{mm}$ and an angle of incidence of $15^{\circ}$, the effect of the mean flow velocity is investigated below. The PIV results for the velocity and vorticity fields for a mean velocity of $10 \mathrm{~m} / \mathrm{s}$ are shown in Figure 44 and Figure 45; the results for a velocity of $15 \mathrm{~m} / \mathrm{s}$ are shown in Figure 46 and Figure 47; the results for a velocity of $25 \mathrm{~m} / \mathrm{s}$ are shown in Figure 48 and Figure 49; and the results for a velocity of $30 \mathrm{~m} / \mathrm{s}$ are shown in Figure 50 and Figure 51. The $20 \mathrm{~m} / \mathrm{s}$ case was previously shown in Figure 37 and Figure 38. A side by side comparison of the average velocity and vorticity fields for a velocity of $10 \mathrm{~m} / \mathrm{s}$ to $30 \mathrm{~m} / \mathrm{s}$ is shown in Figure 52.

For the $10 \mathrm{~m} / \mathrm{s}$ case, no tonal noise was produced, so phase-locked images could not be generated. Therefore, the average velocity and vorticity fields are shown. The velocity and vorticity fields for the $15 \mathrm{~m} / \mathrm{s}$ and $25 \mathrm{~m} / \mathrm{s}$ cases are very similar to that of the $20 \mathrm{~m} / \mathrm{s}$ case. In all three, clear, coherent vortex pairs are shed. For the $30 \mathrm{~m} / \mathrm{s}$ case, the individual vortices become less coherent and periodic. This corresponds to the results of the acoustic measurements in Figure 30, which showed that at $30 \mathrm{~m} / \mathrm{s}$, the magnitude of the main tone decreased and a second peak emerged. Therefore, the phase-locked vorticity, which is locked to the frequency of the main peak, becomes less coherent as the magnitude of the peak decreases. 


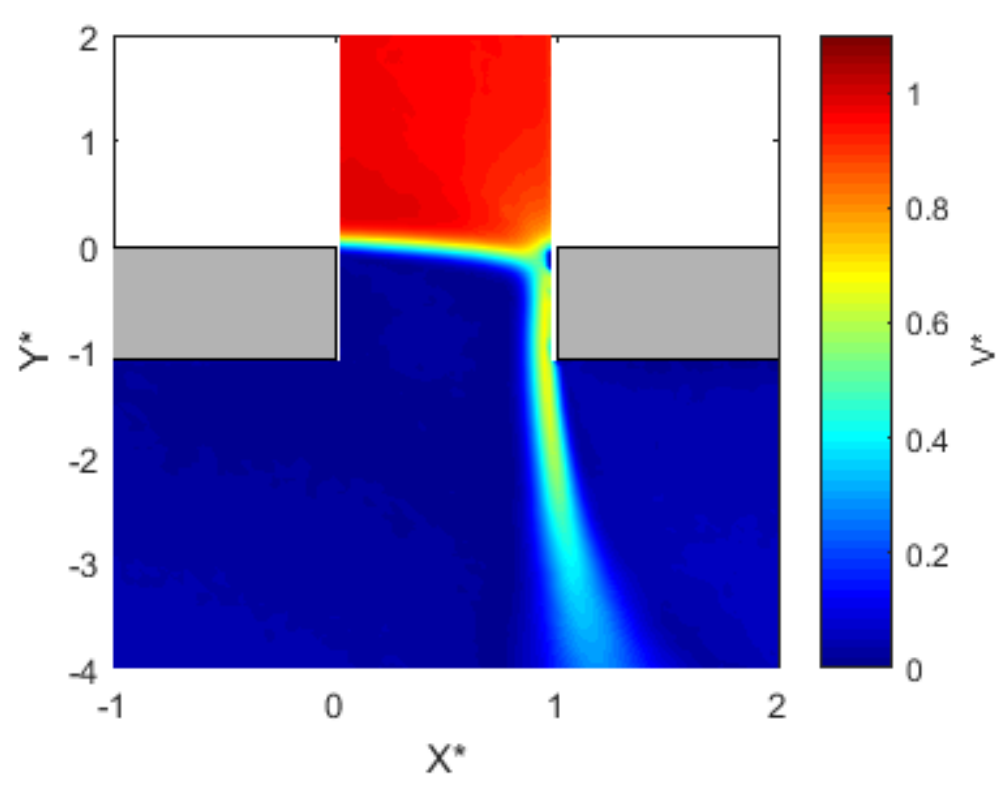

Figure 44: PIV results showing the average velocity field at the 24 th gap for a gap width of $6.35 \mathrm{~mm}$, an angle of incidence of 15 degrees, and a flow velocity of $10 \mathrm{~m} / \mathrm{s}$.

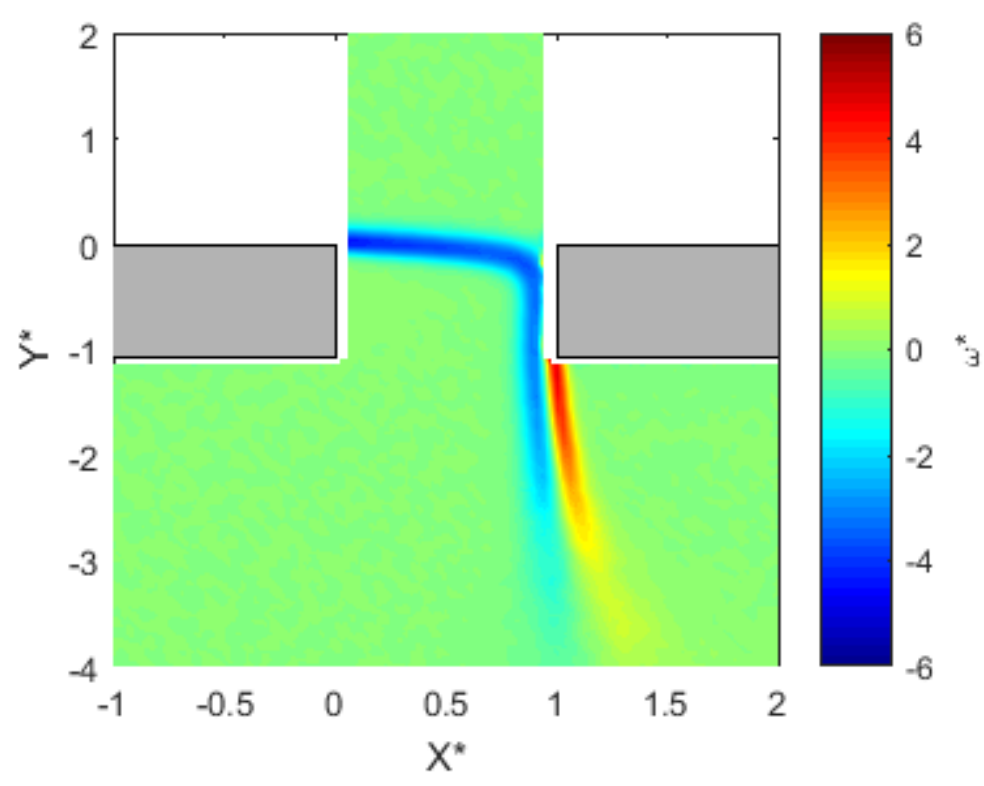

Figure 45: PIV results showing the average vorticity field at the 24th gap for a gap width of $6.35 \mathrm{~mm}$, an angle of incidence of 15 degrees, and a flow velocity of $10 \mathrm{~m} / \mathrm{s}$. 

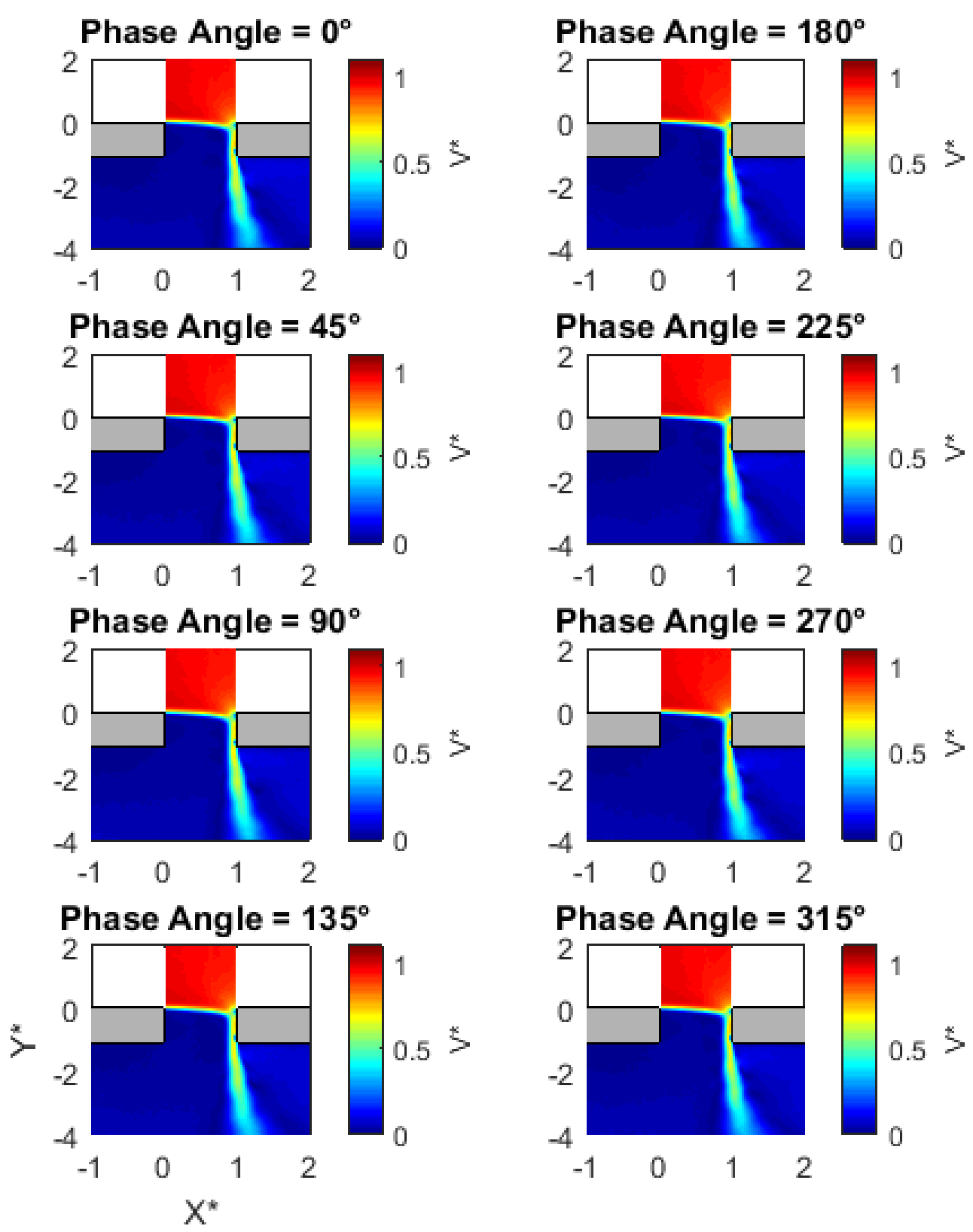

Figure 46: PIV results showing the phase-locked velocity field at the 24th gap for a gap width of $6.35 \mathrm{~mm}$, an angle of incidence of 15 degrees, and a flow velocity of 15 $\mathrm{m} / \mathrm{s}$. 

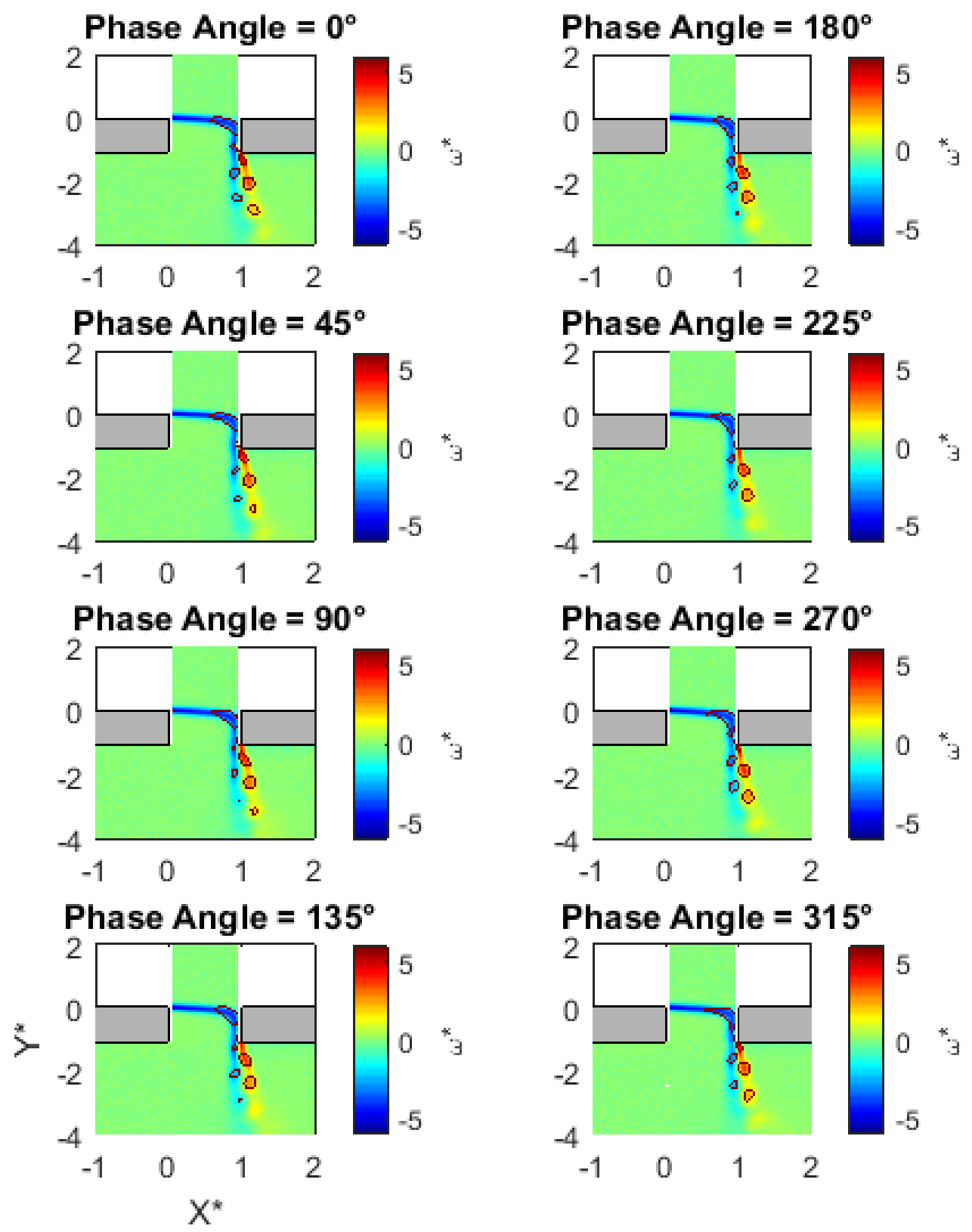

Figure 47: PIV results showing the phase-locked vorticity field at the 24th gap for a gap width of $6.35 \mathrm{~mm}$, an angle of incidence of 15 degrees, and a flow velocity of 15 $\mathrm{m} / \mathrm{s}$. 

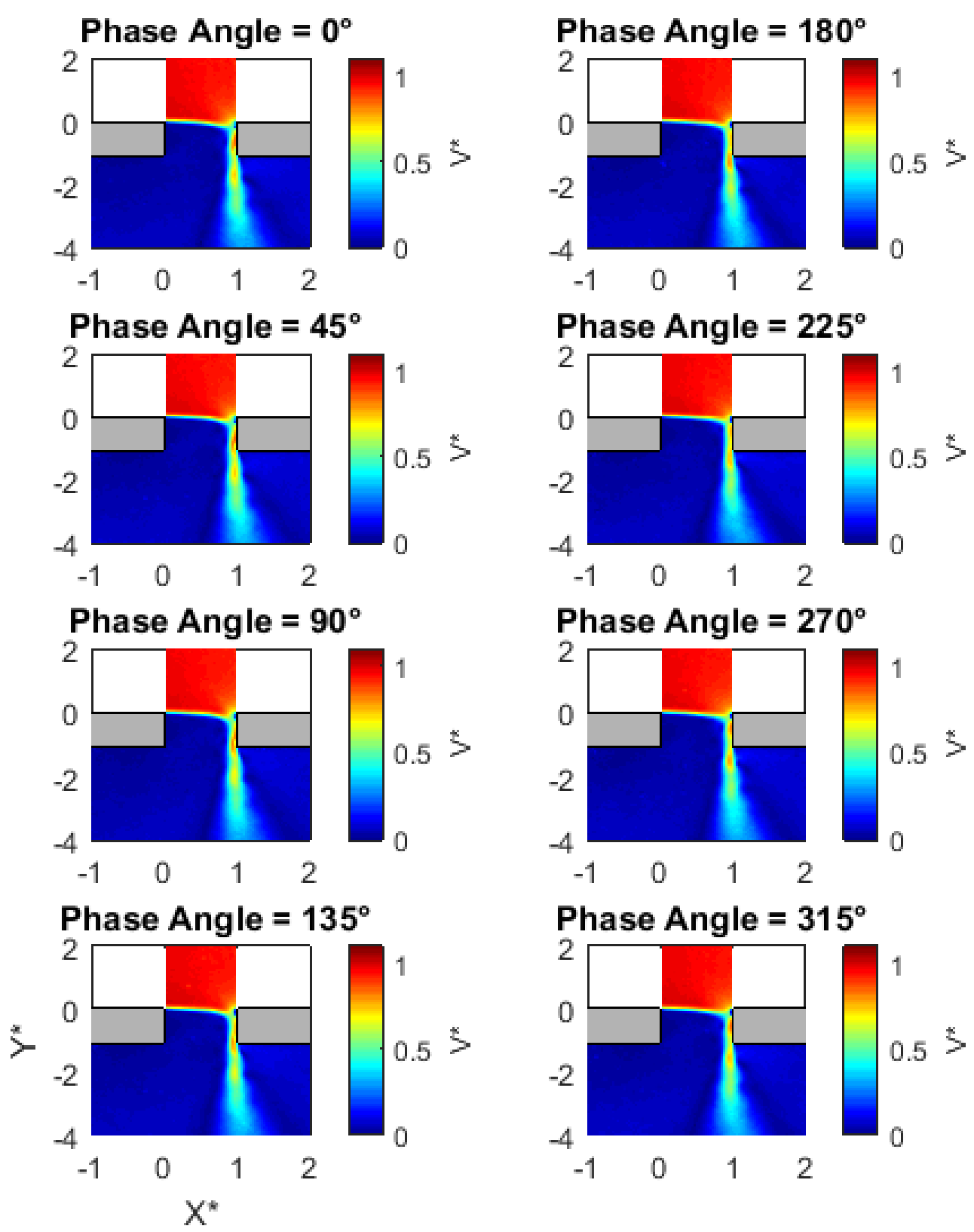

Figure 48: PIV results showing the phase-locked velocity field at the 24th gap for a gap width of $6.35 \mathrm{~mm}$, an angle of incidence of 15 degrees, and a flow velocity of 25 $\mathrm{m} / \mathrm{s}$. 

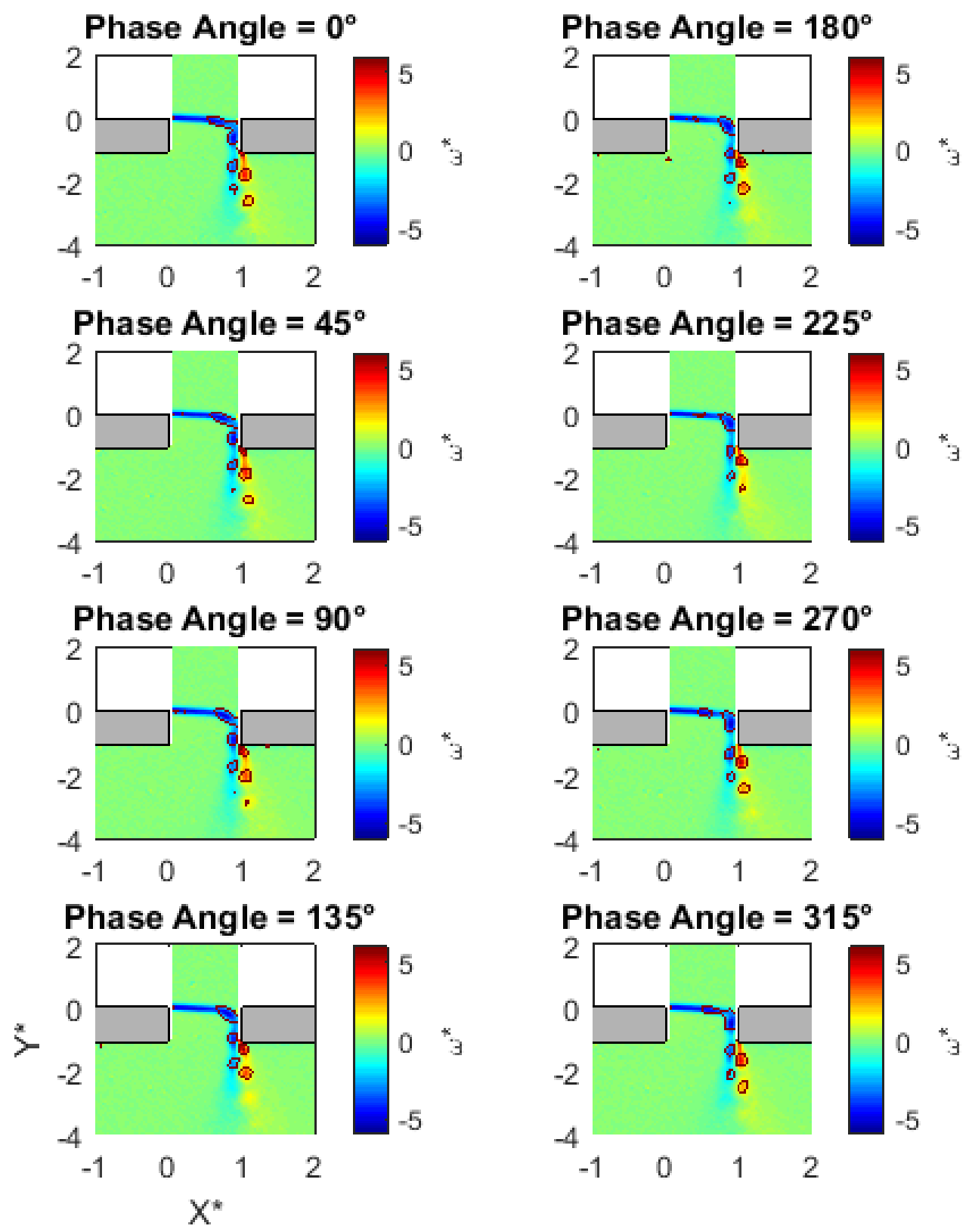

Figure 49: PIV results showing the phase-locked vorticity field at the 24th gap for a gap width of $6.35 \mathrm{~mm}$, an angle of incidence of 15 degrees, and a flow velocity of 25 $\mathrm{m} / \mathrm{s}$. 

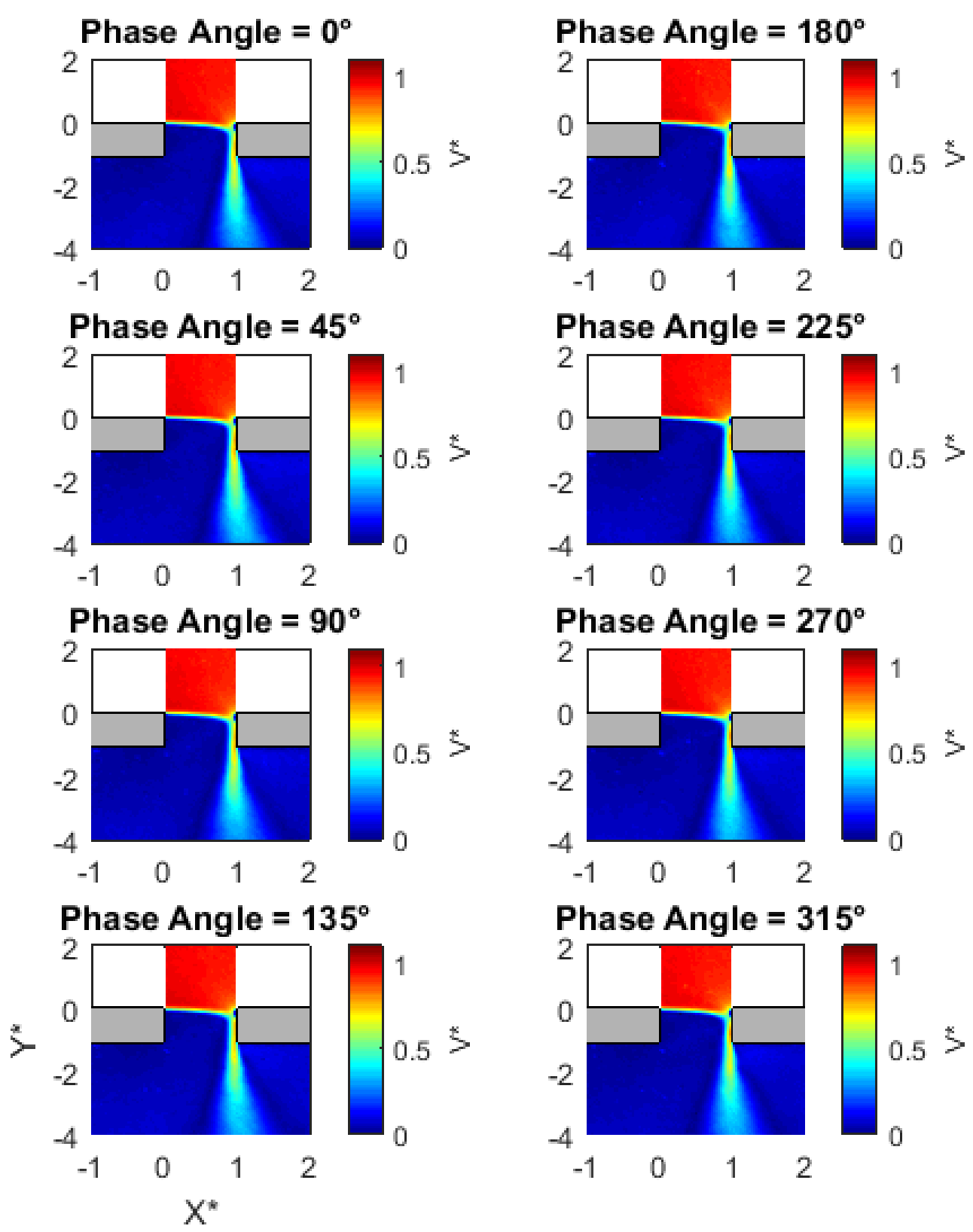

Figure 50: PIV results showing the phase-locked velocity field at the 24th gap for a gap width of $6.35 \mathrm{~mm}$, an angle of incidence of 15 degrees, and a flow velocity of 30 $\mathrm{m} / \mathrm{s}$. 

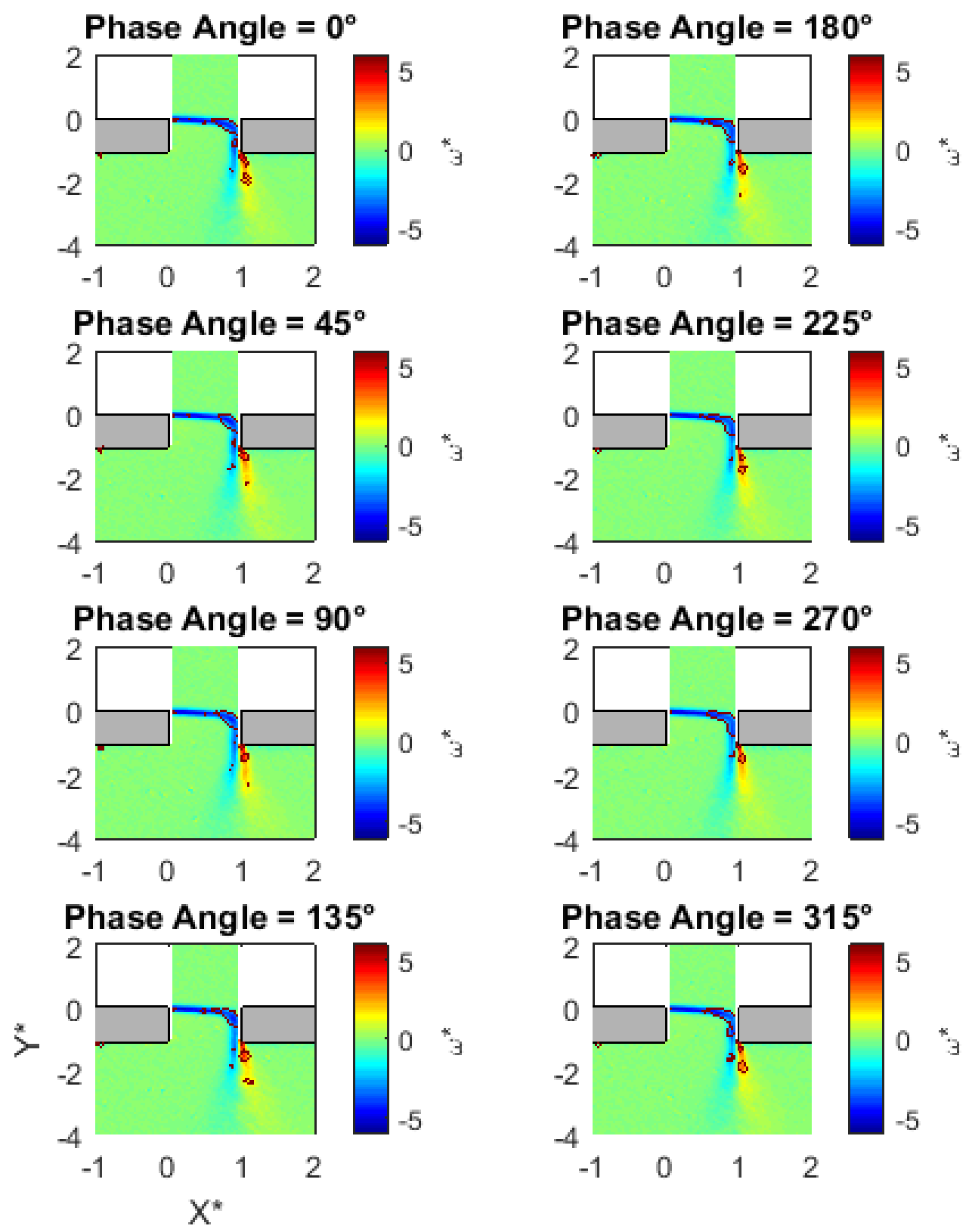

Figure 51: PIV results showing the phase-locked vorticity field at the 24th gap for a gap width of $6.35 \mathrm{~mm}$, an angle of incidence of 15 degrees, and a flow velocity of 30 $\mathrm{m} / \mathrm{s}$. 


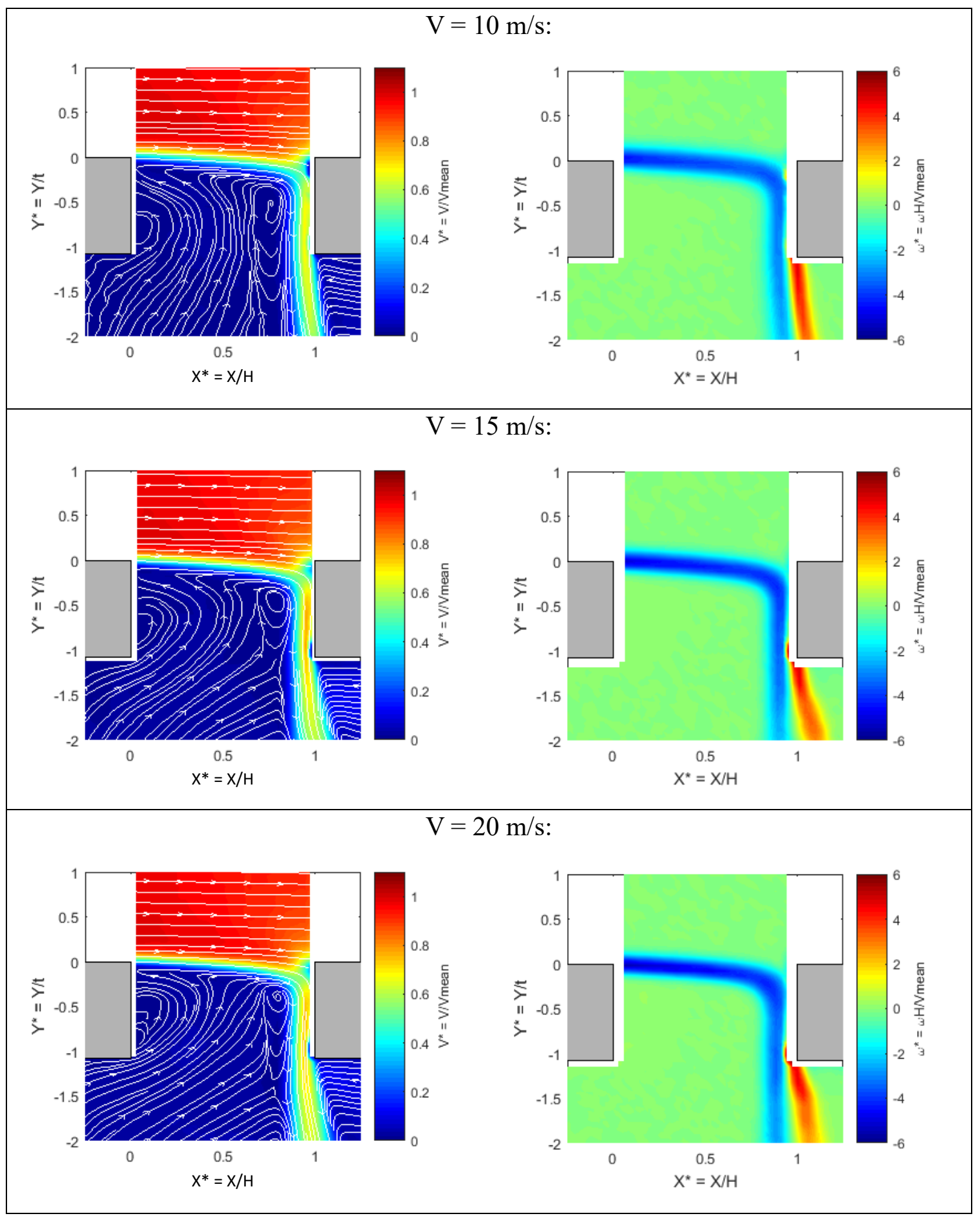

Figure 52: PIV results showing the average velocity (left) and vorticity (right) fields at the 24th gap for a gap width of $6.35 \mathrm{~mm}$, an angle of incidence of 15 degrees, and a flow velocity of 10 to $30 \mathrm{~m} / \mathrm{s}$. The white lines are streamlines. (continued on the following page) 


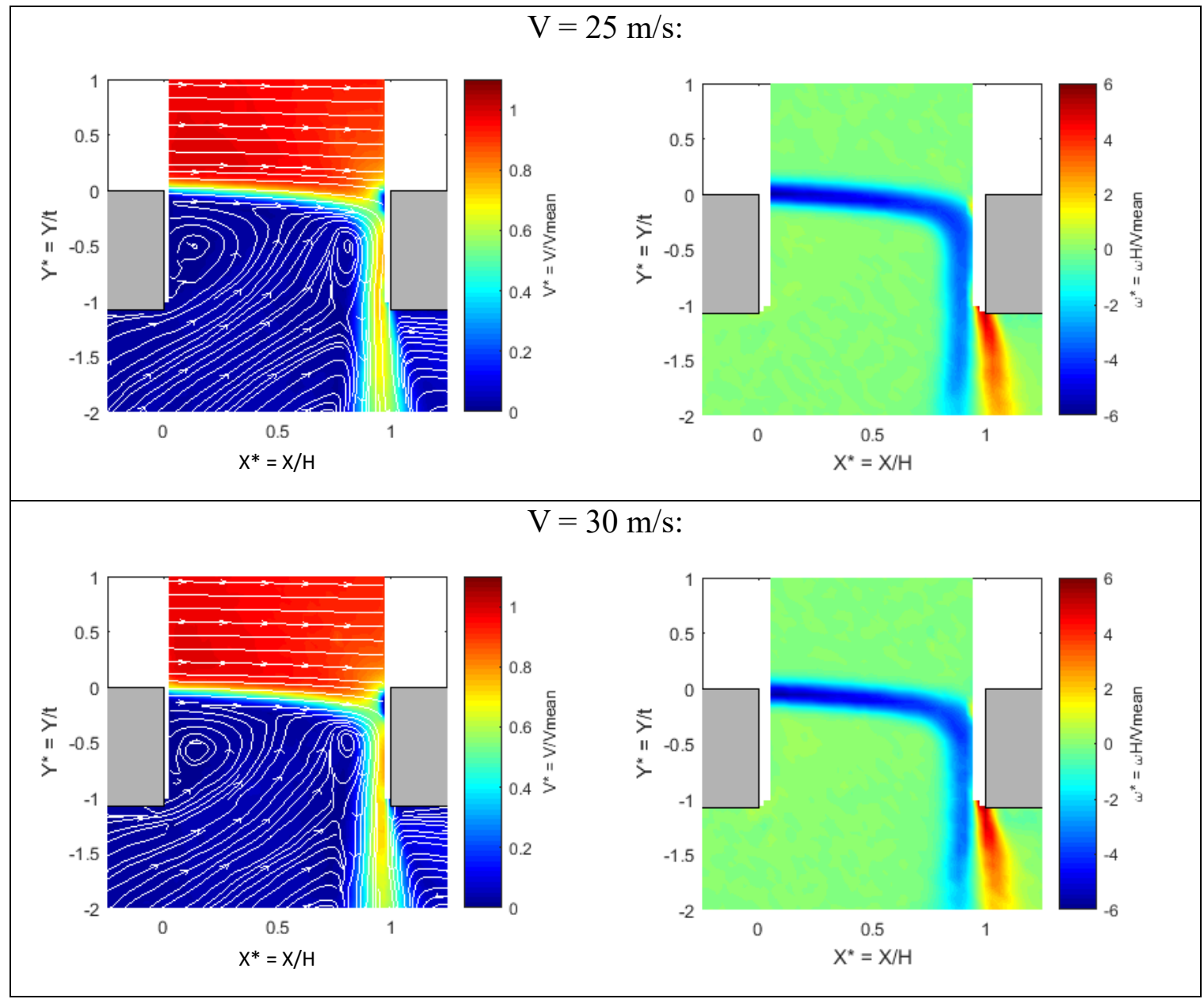

Figure 52 (continued): PIV results showing the average velocity (left) and vorticity (right) fields at the 24th gap for a gap width of $6.35 \mathrm{~mm}$, an angle of incidence of 15 degrees, and a flow velocity of 10 to $30 \mathrm{~m} / \mathrm{s}$. The white lines are streamlines.

Figure 53 below shows the vorticity field at a single phase for the flow velocities $15,20,25$, and $30 \mathrm{~m} / \mathrm{s}$. Phases were selected which are at approximately the same point in the acoustic cycle. At the velocities 15, 20, and $25 \mathrm{~m} / \mathrm{s}$, a total of seven vortices were identified by the $d_{2}$ parameter. At $30 \mathrm{~m} / \mathrm{s}$, only five vortices were identified. The vortices in this case appear to be weaker and dissipate more quickly. 


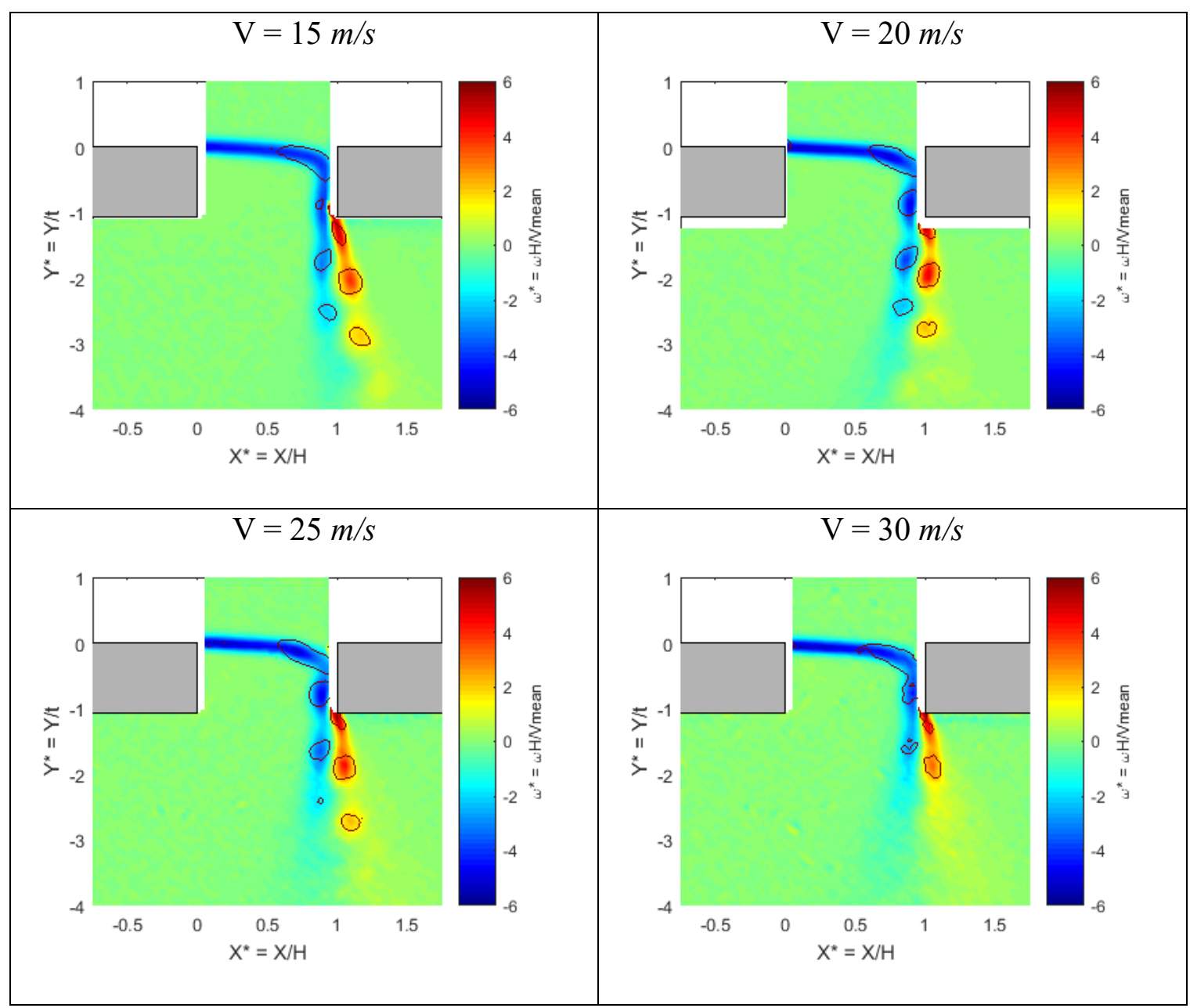

Figure 53: PIV results showing the phase-locked vorticity field at a single phase at the $24^{\text {th }}$ gap for a gap width of $6.35 \mathrm{~mm}$, an angle of incidence of 15 degrees, and a flow velocity of 15 to $30 \mathrm{~m} / \mathrm{s}$.

Using the velocity field data from the PIV results, the Strouhal number based on momentum thickness, $S t_{\Theta}$, is calculated. This is plotted in Figure 54 as a function of the $X$ position along the mouth of the gap. The value of $S t_{\Theta}$ is calculated using equation (2) at discrete points corresponding to the pixel locations in the PIV results. Because the momentum thickness is found based on the $u$-component of the velocity field, it becomes undefined once there is a significant $v$-component to the velocity. This is the case for $X^{*}$ 
greater than 0.4. This corresponds to the downstream side of the gaps, where the air is flowing through to the backside of the plate with a significant negative $v$-component. Therefore, Figure 54 shows the momentum thickness data only for the upstream portion of the gap, up to $X^{*}=0.4$. Initial data near the upstream corner is also omitted because of the uncertainty of the PIV results near the wall. Therefore, the data in Figure 54 starts at $X^{*}=$ 0.05 .

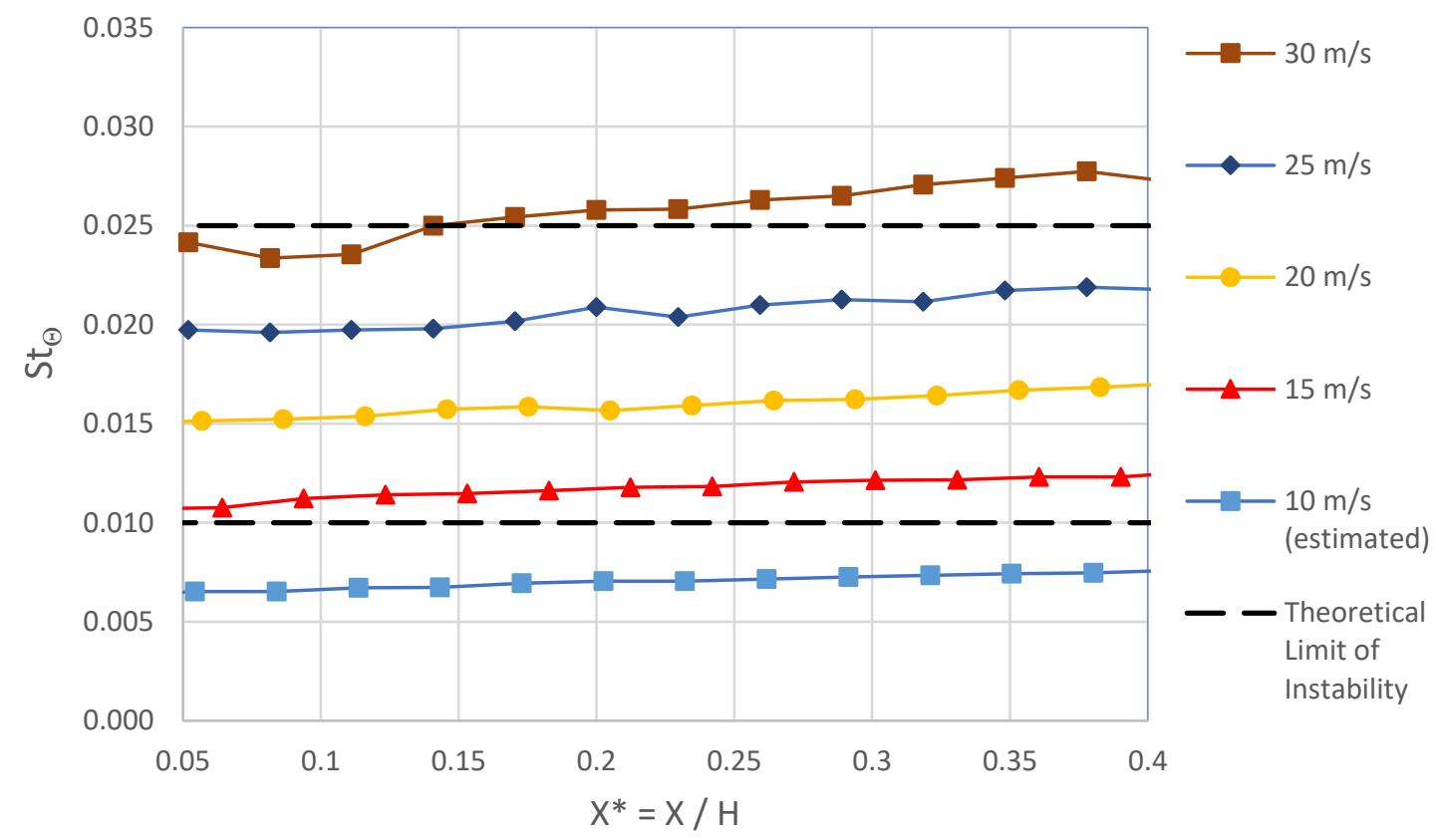

Figure 54: Strouhal number based on momentum thickness, St $\Theta$, as a function of $X^{*}$. Results are shown for the $24^{\text {th }}$ gap for a gap width of $6.35 \mathrm{~mm}$, an angle of incidence of 15 degrees, and a flow velocity of 10 to $30 \mathrm{~m} / \mathrm{s}$. The region between the theoretical limits of instability are values of $\mathrm{St}_{\Theta}$ at which the free shear layer is most unstable, based on the work of Michalke (1972).

A free shear layer is most unstable and prone to spatially growing disturbances when its Strouhal number based on momentum thickness falls between 0.01 and 0.025 (Michalke, 1972), which is indicated by the dashed lines in Figure 54. The maximum instability is 
found around $S t_{\Theta}=0.0176$. The Strouhal number for all the velocities shown falls between the limits of instability, except for the $10 \mathrm{~m} / \mathrm{s}$ case, which falls below the limit, and the 30 $\mathrm{m} / \mathrm{s}$ case, which falls slightly above the limit. The $15 \mathrm{~m} / \mathrm{s}$ case falls just above the lower limit, while the $20 \mathrm{~m} / \mathrm{s}$ and $25 \mathrm{~m} / \mathrm{s}$ cases are well within the limits of instability. Note that for the $10 \mathrm{~m} / \mathrm{s}$ case, no tones were produced, so the frequency used in the calculation of $S t_{\Theta}$ was estimated by linearly extrapolating the peak frequencies for the $15 \mathrm{~m} / \mathrm{s}, 20 \mathrm{~m} / \mathrm{s}, 25 \mathrm{~m} / \mathrm{s}$, and $30 \mathrm{~m} / \mathrm{s}$ cases.

The findings of Figure 54 agree well with the acoustics and the PIV results. The produced tonal noise was found to have the greatest magnitude for a velocity of 20 to 25 $\mathrm{m} / \mathrm{s}$, where the free shear layer has been found to be the most unstable. The magnitude of the produced tones decreased as the velocity was increased above $25 \mathrm{~m} / \mathrm{s}$ or decreased below $20 \mathrm{~m} / \mathrm{s}$, as the free shear layer moves closer to the upper and lower limits of instability. In the PIV results, it was seen that the shed vortices in the 15,20 , and $25 \mathrm{~m} / \mathrm{s}$ cases are stronger and remain coherent for longer than in the $10 \mathrm{~m} / \mathrm{s}$ or $30 \mathrm{~m} / \mathrm{s}$ cases. 


\subsubsection{Effect of streamwise position}

It was found in the acoustic results that the noise level emitted from the slats increased along the slats in the streamwise direction (see Figure 33 and Figure 34). For the case of a $6.35 \mathrm{~mm}$ gap width, 15 degree angle of incidence, and $20 \mathrm{~m} / \mathrm{s}$ flow velocity, the magnitude of the tones increased until around the $15^{\text {th }}$ gap, then remained approximately constant until around the $27^{\text {th }}$ gap, before decreasing again past the $27^{\text {th }}$ gap.

PIV images were taken at the $6^{\text {th }}, 12^{\text {th }}, 18^{\text {th }}$, and $24^{\text {th }}$ gap locations to study the development of the flow along the plate. Figure 55 and Figure 56 show the velocity and vorticity fields for a $6.35 \mathrm{~mm}$ gap width, a 15-degree angle of incidence, and a $20 \mathrm{~m} / \mathrm{s}$ flow velocity, taken at the $18^{\text {th }}$ gap. These are the same input conditions as Figure 37 and Figure 38 , which were taken at the $24^{\text {th }}$ gap. It is seen that at the $18^{\text {th }}$ gap, there is still a streak of negative vortices originating from the upstream slat and a streak of positive vortices originating from the backside of the downstream slat. Similar to the $24^{\text {th }}$ gap, these vortices are shed periodically from the trailing edge of the downstream slat. However, the individual vortices are less coherent than they are at the $24^{\text {th }}$ gap location.

Figure 57 and Figure 58 show the velocity and vorticity fields at the $12^{\text {th }}$ gap location. In comparison to the $18^{\text {th }}$ gap, the vortices shed from the backside of the downstream slat are less coherent and dissipate more quickly. A similar trend is seen in Figure 59 and Figure 60 for the $6^{\text {th }}$ gap location.

This agrees well with the acoustic results. Going from the $6^{\text {th }}$ to the $12^{\text {th }}, 18^{\text {th }}$, and $24^{\text {th }}$ gaps, the shed vortices become increasingly periodic and coherent. This corresponds to the increase in magnitude of the acoustic tones in the streamwise direction. 

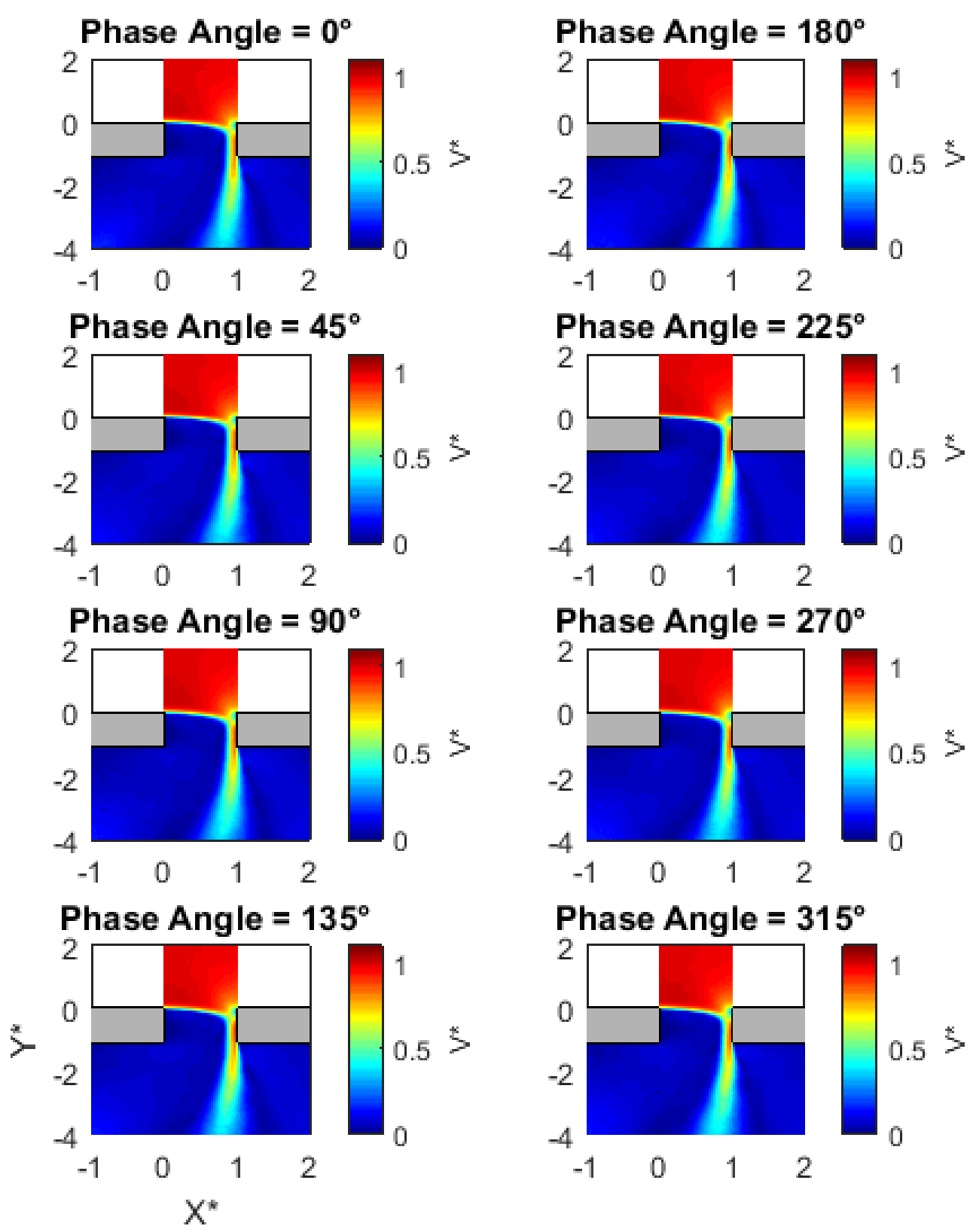

Figure 55: PIV results showing the phase-locked velocity field at the 18th gap for a gap width of $6.35 \mathrm{~mm}$, an angle of incidence of 15 degrees, and a flow velocity of 20 $\boldsymbol{m} / \mathbf{s}$. 

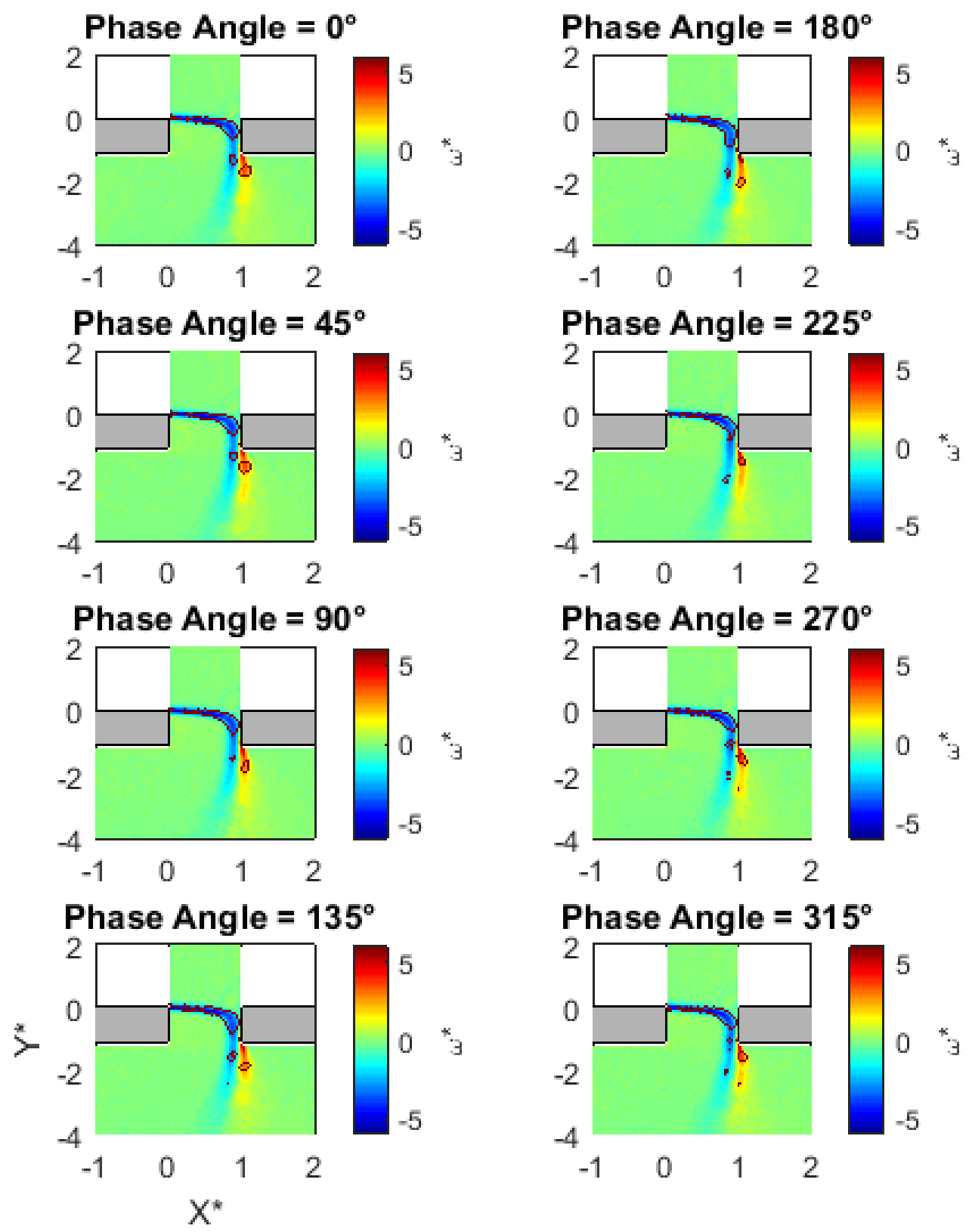

Figure 56: PIV results showing the phase-locked vorticity field at the 18th gap for a gap width of $6.35 \mathrm{~mm}$, an angle of incidence of 15 degrees, and a flow velocity of 20 $\mathrm{m} / \mathrm{s}$. 

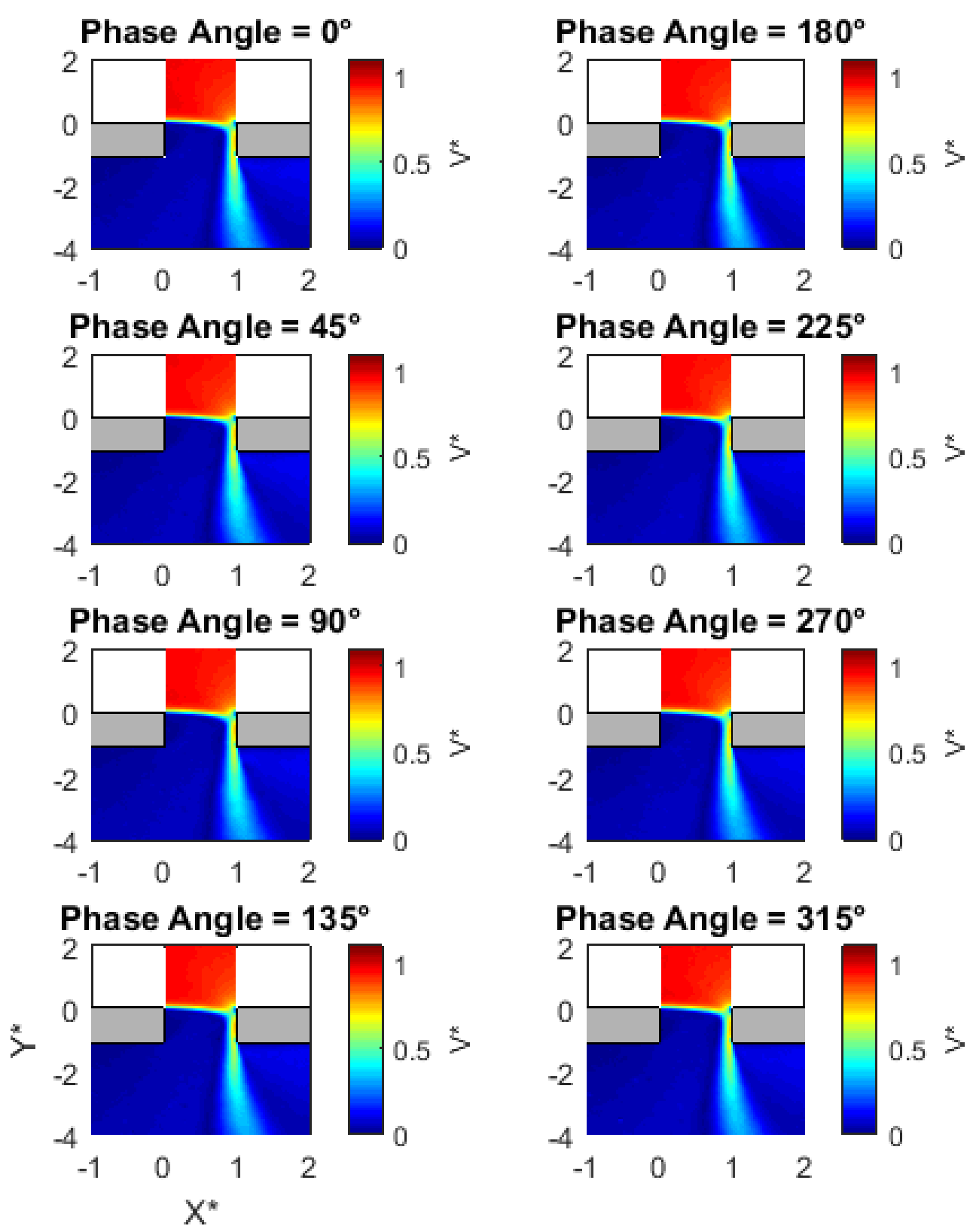

Figure 57: PIV results showing the phase-locked velocity field at the 12th gap for a gap width of $6.35 \mathrm{~mm}$, an angle of incidence of 15 degrees, and a flow velocity of 20 $\boldsymbol{m} / \mathbf{s}$. 

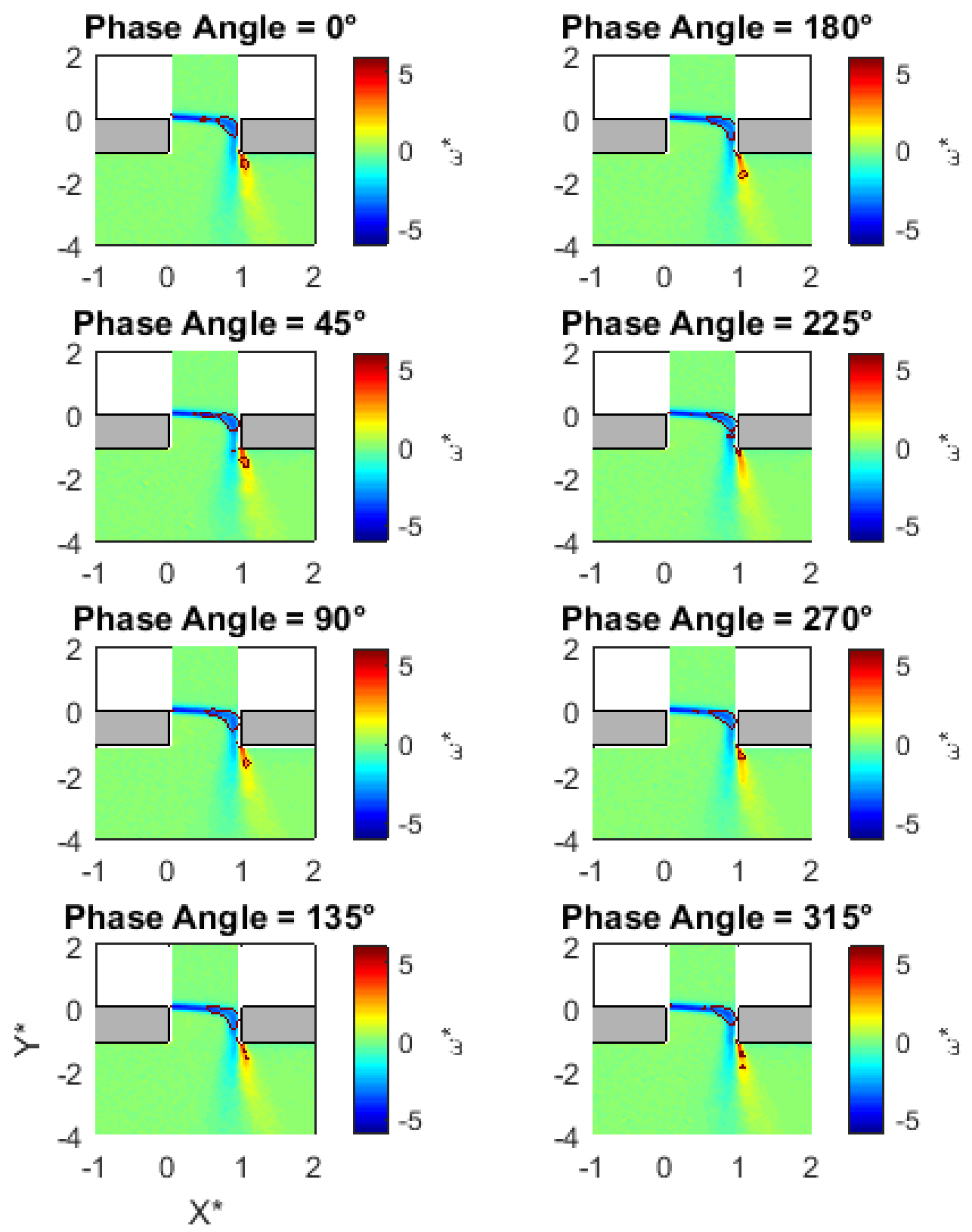

Figure 58: PIV results showing the phase-locked vorticity field at the 12th gap for a gap width of $6.35 \mathrm{~mm}$, an angle of incidence of 15 degrees, and a flow velocity of 20 $\mathrm{m} / \mathrm{s}$. 

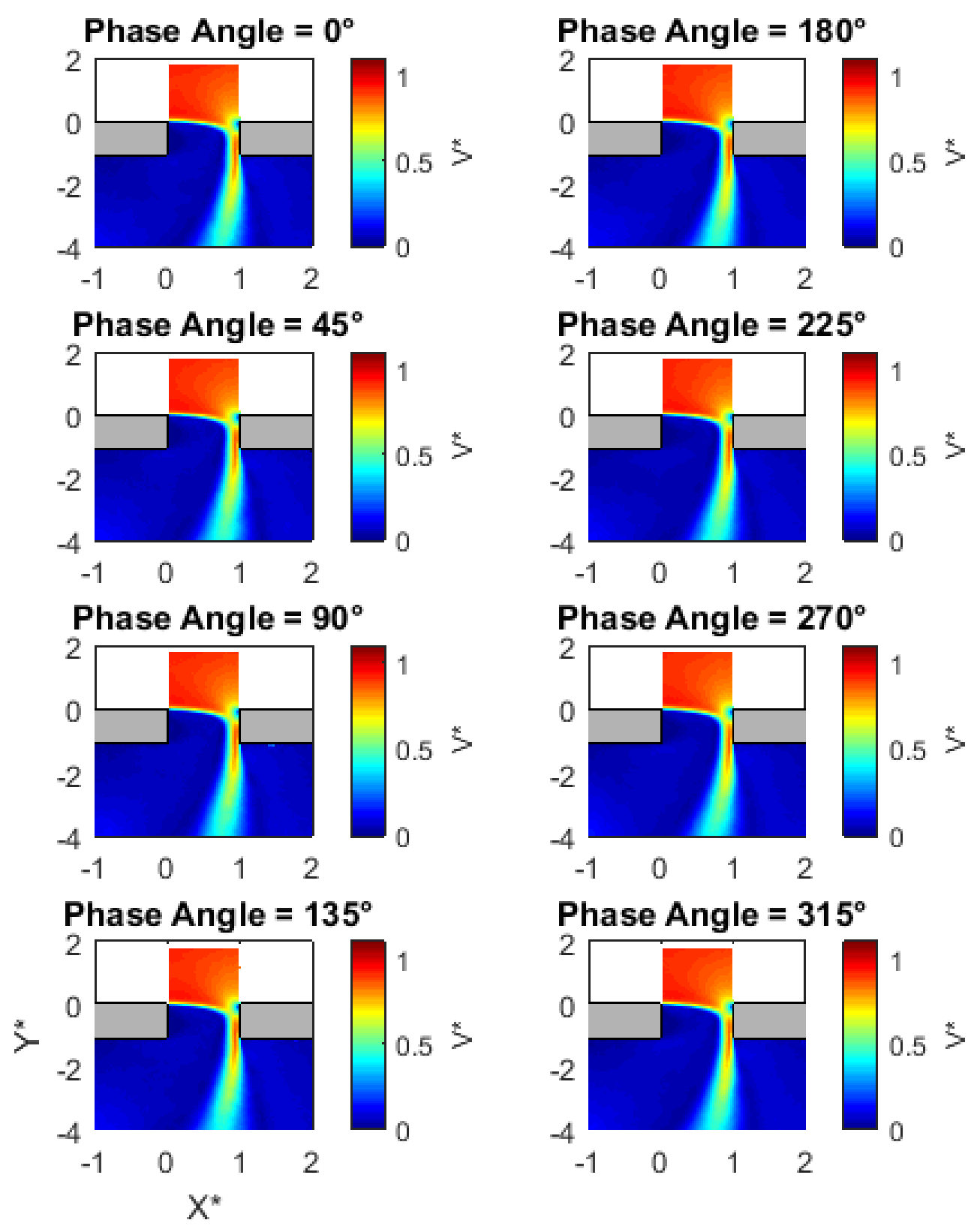

Figure 59: PIV results showing phase-locked velocity field at the 6th gap for a gap width of $6.35 \mathrm{~mm}$, an angle of incidence of 15 degrees, and a flow velocity of $20 \mathrm{~m} / \mathrm{s}$. 

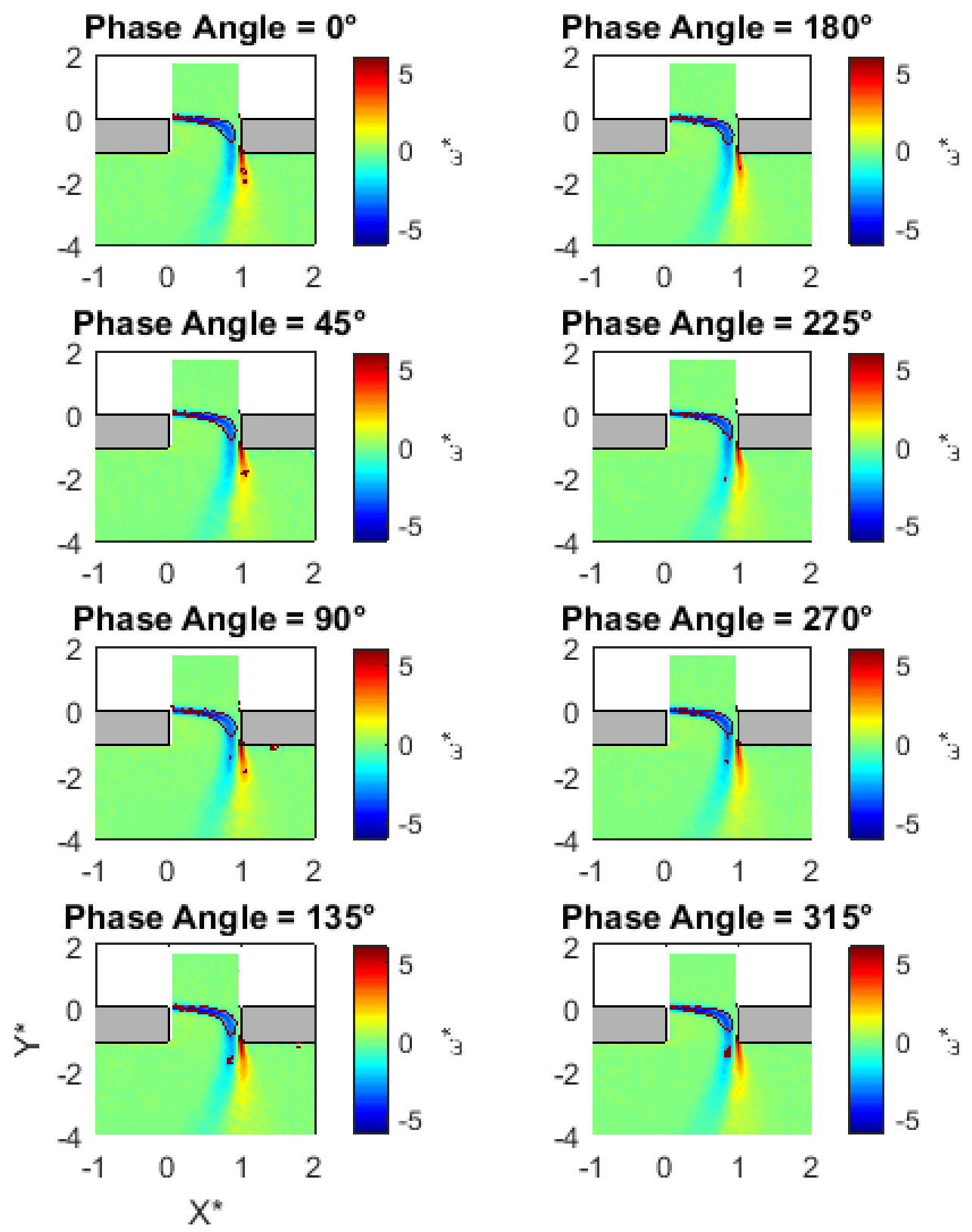

Figure 60: PIV results showing the phase-locked vorticity field at the 6th gap for a gap width of $6.35 \mathrm{~mm}$, an angle of incidence of 15 degrees, and a flow velocity of 20 $\mathrm{m} / \mathrm{s}$. 
Figure 61 compares the mean velocity and vorticity fields at the $6^{\text {th }}, 12^{\text {th }}, 18^{\text {th }}$, and $24^{\text {th }}$ gap locations. At the $6^{\text {th }}$ gap, the local angle of incidence of the flow is the greatest. This results in a large stagnation region at the $\left(X^{*}=1, Y^{*}=0\right)$ corner of the gap. At the $12^{\text {th }}, 18^{\text {th }}$, and $24^{\text {th }}$ gaps, the local angle of incidence decreases and this stagnation region becomes smaller. A recirculation zone forms at around $\left(X^{*}=0.75, Y^{*}=-0.5\right)$. This is most pronounced at the $24^{\text {th }}$ gap, where the most coherent vortices are observed.

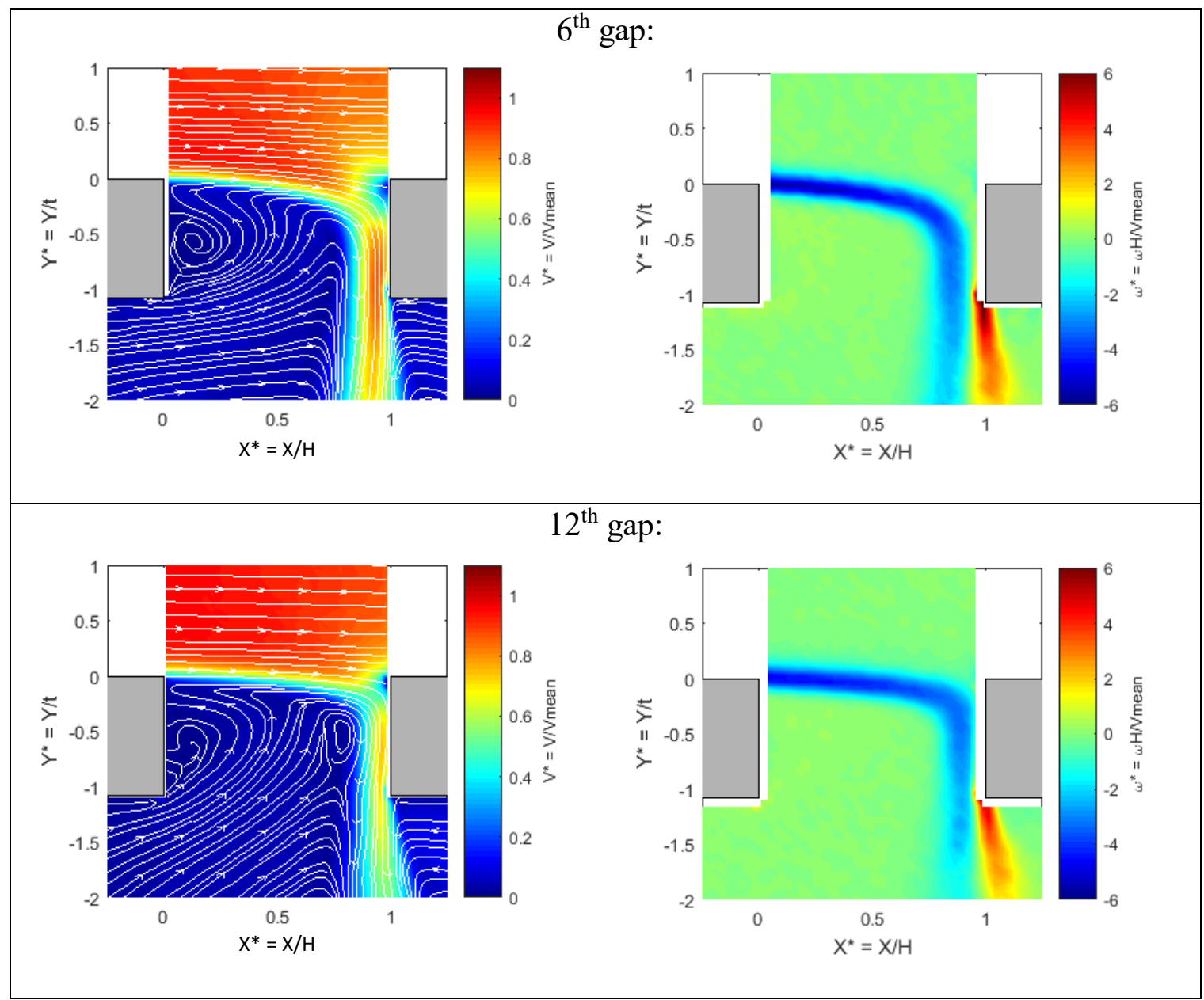

Figure 61: PIV results showing the average velocity (left) and vorticity (right) fields for a gap width of $6.35 \mathrm{~mm}$, an angle of incidence of 15 degrees, and a flow velocity of $20 \mathrm{~m} / \mathrm{s}$ at the $6^{\text {th }}, 12^{\text {th }}, 18^{\text {th }}$, and $24^{\text {th }}$ gap locations. The white lines are streamlines. (continued on the following page) 


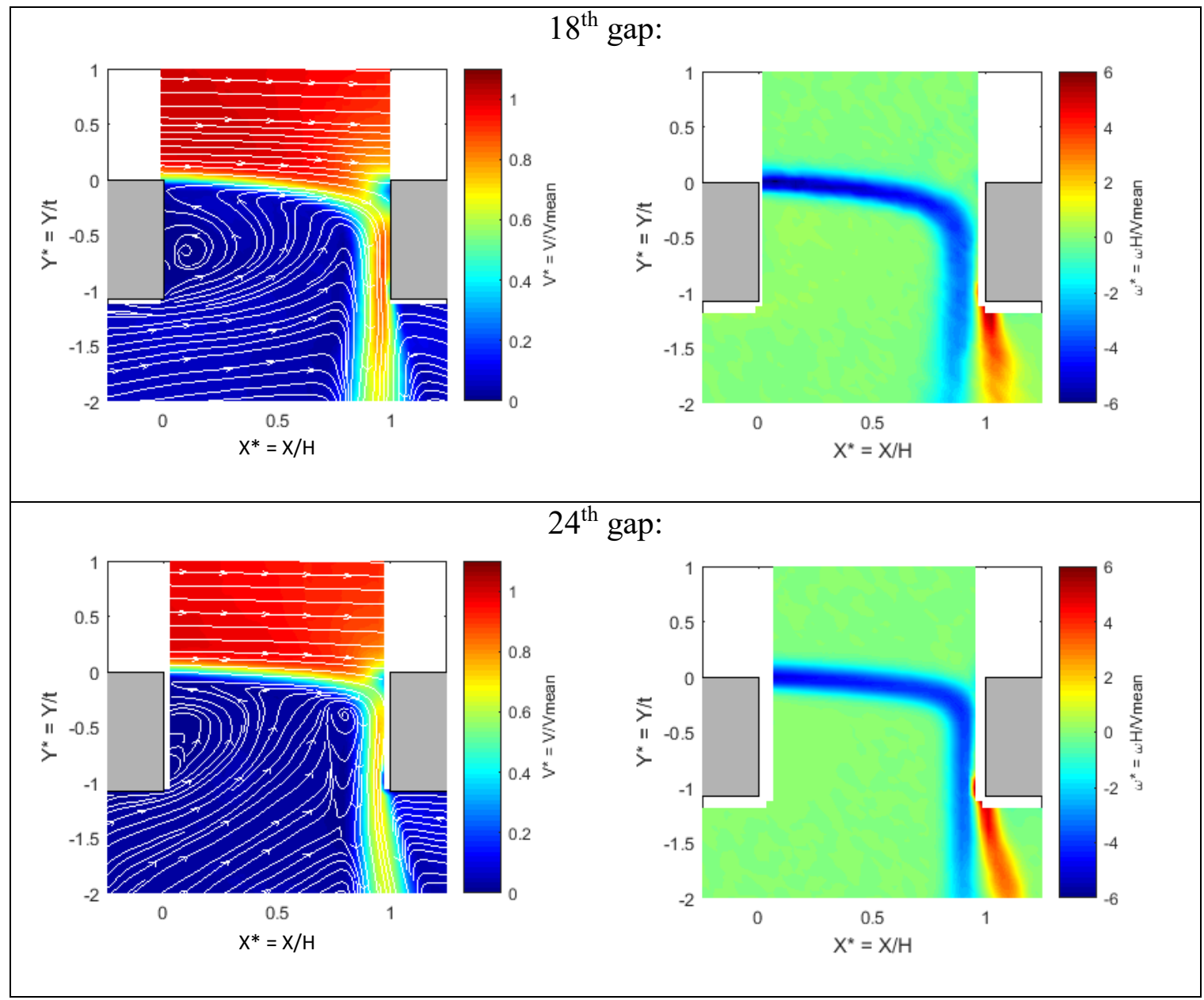

Figure 61 (continued): PIV results showing the average velocity (left) and vorticity (right) fields for a gap width of $6.35 \mathrm{~mm}$, an angle of incidence of 15 degrees, and a flow velocity of $20 \mathrm{~m} / \mathrm{s}$ at the $6^{\text {th }}, 12^{\text {th }}, 18^{\text {th }}$, and $24^{\text {th }}$ gap locations. The white lines are streamlines.

Figure 62 shows the vorticity field at a single phase for the $6^{\text {th }}, 12^{\text {th }}, 18^{\text {th }}$, and $24^{\text {th }}$ gaps. Phases were selected which are at approximately the same point in the acoustic cycle. At the $24^{\text {th }}$ gap, a total of seven vortices were identified by the $d_{2}$ parameter, at various stages of their propagation. These vortices are very strong and coherent. At the $18^{\text {th }}$ gap, only four vortices were identified, two positive and two negative. At the $12^{\text {th }}$ gap, only three 
vortices were identified, and at the $6^{\text {th }}$ gap, no clear and distinct vortices were identified. The vortices become progressively weaker and dissipate more quickly further upstream.

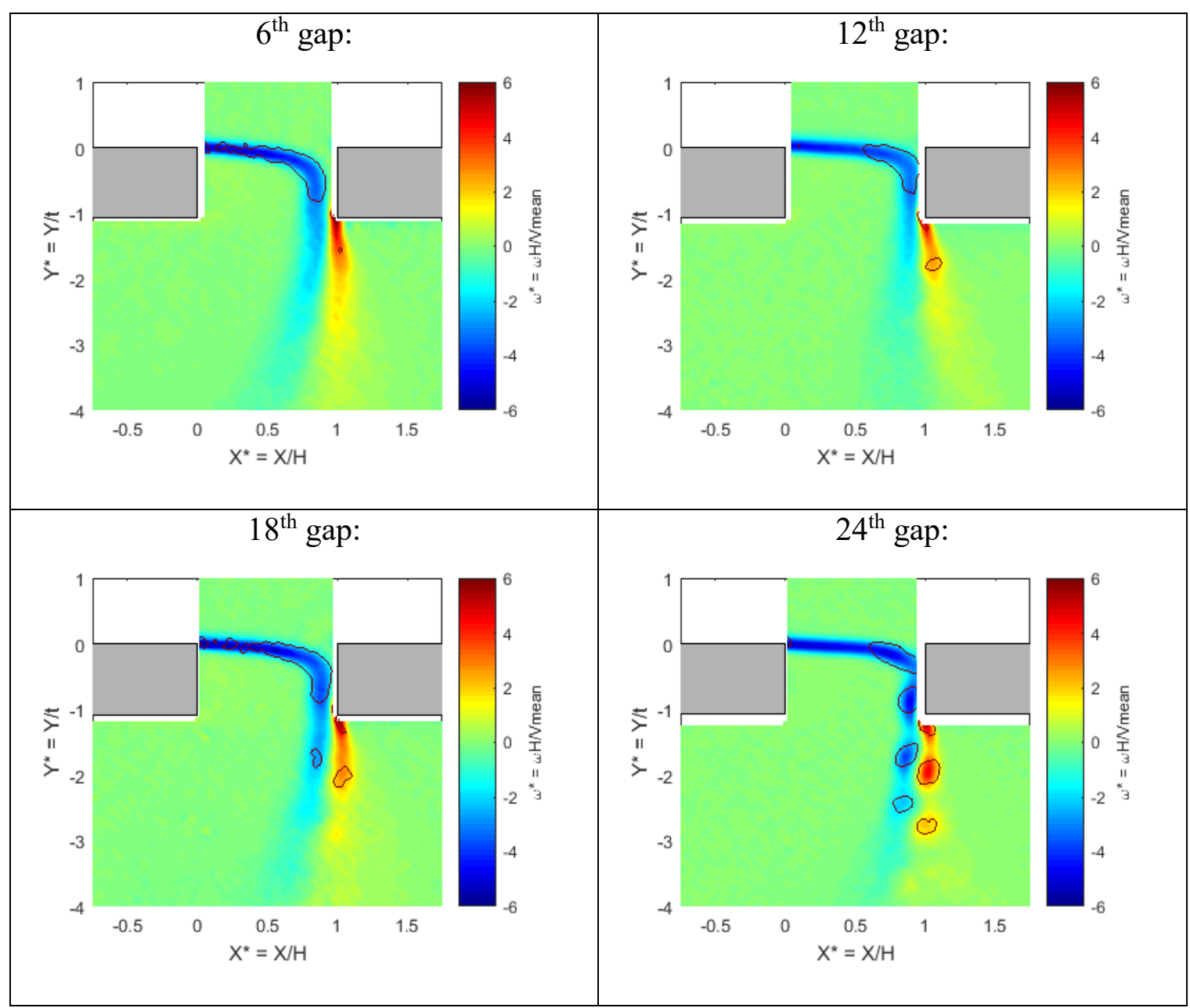

Figure 62: PIV results showing the phase-locked vorticity field at a single phase at the for a gap width of $6.35 \mathrm{~mm}$, an angle of incidence of 15 degrees, and a flow velocity of $20 \mathrm{~m} / \mathrm{s}$, at the $6^{\text {th }}, 12^{\text {th }}, 18^{\text {th }}$, and $24^{\text {th }}$ gap locations.

As the flow moves along the length of the plate, the flow conditions change, becoming more favorable to the production of tones. The velocity and angle of incidence of the flow are fixed at the upstream end of the plate, but they can change as the flow moves 
downstream. In particular, there is an increase in the grazing component ( $X$-component) of the flow and a decrease in the normal component ( $Y$-component). This causes the local angle of incidence to decrease. This is seen for the $6^{\text {th }}$ gap in Figure 61 , where the stagnation region on the downstream slat is larger and a greater portion of the fluid passes through the gap. In the vorticity plots, the shear layer at the $6^{\text {th }}$ gap is more rounded near the $\left(X^{*}=1\right.$, $Y^{*}=0$ ) corner. On the other hand, at the $24^{\text {th }}$ gap, the local angle of incidence is smaller and the shear layer turns sharply downward at the $\left(X^{*}=1, Y^{*}=0\right)$ corner. This results in a stronger feedback and higher amplitude tonal noise. The optimal condition for tone generation appear to be when the local angle of incidence is large enough that the shear layer does not graze over the downstream corner, but small enough that it impinges near this corner and turns sharply downward. Under this condition, the impingement on the downstream slat is nearly at a perpendicular direction. If the local angle of incidence is increased and the impingement point is lower down on the downstream slat, the shear layer impinges at an angle less than 90 degrees and the feedback is not as strong. Tones are still produced in this case, but they are relatively weaker. 


\section{CHAPTER 5 CONCLUSIONS}

\subsection{Summary of Results}

It has been shown that flow over perforated plates at oblique angles of incidence produces tonal noise by the fluid dynamic feedback mechanism. A two-dimensional model of a perforated plate was used, where the circular holes were replaced with rectangular gaps. The use of this model was validated by comparing to the results for a perforated plate of similar geometry.

The experimental apparatus consisted of a series of rectangular slats mounted to a frame, which pivoted at the exit of an open loop wind tunnel. The studied input parameters were the angle of incidence of the flow, the flow velocity, the gap width, and the streamwise position. Microphone measurements were taken to study how each of these inputs affects the produced noise, and particle image velocimetry flow visualization was done to explain the tone generation mechanism. It was found that the local angle of incidence of the flow develops along the length of the plate. The flow conditions become more favorable to the production of tonal noise as the flow develops, resulting in an increase in the magnitude of the produced noise in the streamwise direction.

For a given plate geometry, tones were observed for a specific range of angles of incidence of the flow. The angle must be such that the free shear layer originating at the leading edge of the gap impinges on the downstream side of the gap. The flow then moves down along the downstream side of the gap and counter-rotating vortices are shed at the 
downstream corner. In the unstable free shear layer spanning the gap, vortical structures form. The periodic impingement and shedding of these vortices at the trailing edge of the gap produces audible tonal noise or whistling of the plate. For plates with a larger gap width over thickness ratio, $H / t$, tones are produced at smaller angles of incidence and a narrower range of angles.

Within the optimal range of angles, the frequency of the tones increases linearly with increasing flow velocity, which is typical of the fluid dynamic mechanism. Similar to the angle of incidence, there is a preferred range of flow velocity at which tones are produced. The magnitude of the tones is greatest when the free shear layer is most unstable, which is generally in the range of Strouhal number based on momentum thickness, $S t_{\Theta}$, of 0.1 to 0.25 . Within this range, the vortices which form in the free shear layer are stronger and more coherent, resulting in louder tones. For plates with a larger gap width, tones are produced at lower flow velocities.

The results of this work give some hints to designers when using perforated plates on the exterior faces of buildings. The velocity and direction of the wind are out of the designer's control, but the geometric parameters of the plate can be carefully selected to prevent wind-induced noise problems. The hole diameter can be selected based on the range of wind velocities which the plate is expected to be exposed to. One approach is to select a smaller hole diameter, such that the maximum expected wind velocity is not great enough to produce tones. A second approach is to select a larger hole diameter, such that tones are produced at low wind velocities, but the magnitude of these tones is low enough to not be an irritant. The hole diameter and plate thickness can also be selected together to control 
the $D / t$ ratio. For a larger $D / t$ ratio, tones will be produced for a narrower range of angles of incidence. On a building, this may result in less frequent whistling. Finally, the fact that the magnitude of the tones increased along the plate in the streamwise direction may be of use to designers. If the flow can be broken up at an appropriate point along the length of the plate, it may be possible to prevent the noise from reaching its maximum magnitude. This could be done by separating a longer span of perforated plate into several shorter spans or by tripping the flow at an appropriate location.

\subsection{Future Work}

As discussed in Chapter 2, very little work has been done regarding flow over perforated plates at oblique angles of incidence. Therefore, a lot of work can be done to build upon the results of this thesis.

It was found that the magnitude of the noise increases in the streamwise direction along the plate. A more comprehensive study could be done on this effect. Future work could look at different lengths of plates, to better understand how the plate length effects the produced noise. Since the noise increases along the length of the plate, it is possible that the noise can be reduced by breaking up a longer plate into multiple short sections.

The effect of edge rounding can be investigated in more detail. It was found that the frequency of the tones produced by a perforated plate change depending on the roundness of the leading and trailing edges. This could be enhanced by testing plates with different edge roundness. Flow visualization could also be done to see how the mechanism changes with different edge roundness. 
It was also found in the acoustic results that under some conditions, the produced noise consists of multiple tonal peaks. The focus of this work was mainly on the largest peak, at a Strouhal number of around 1 to 1.4. Additional work could be done to look into the mechanism which produces these other peaks, and under what conditions they are produced.

Finally, a more comprehensive parametric study could be completed to develop a complete picture of how the many variables affect the noise production. Some of the variables that could be looked at in more detail include the plate thickness, the gap width, and the spacing between gaps, as well as the edge roundness and plate length, mentioned above. In this thesis, only a few different cases were investigated to develop an understanding of the mechanism. Now that the mechanism is well understand, the effect of these variables should be looked at in more detail to obtain a complete map of how each input affects the produced noise. 


\section{REFERENCES}

Blinet, T., Kerdodou, P., Chene, J.-B., \& Jacqus, G. (2015). Wind Noise Generated by Facade Elements on Buildings: A Simple Measurement Method and Case Studies. Inter-Noise 2015. San Francisco.

Bruggeman, J. C. (1987). Flow-Induced Pulsations in Pipe Systems. Doctoral Dissertation, Techniche Universait.

Celik, E., \& Rockwell, D. (2002). Shear Layer Oscillation Along a Perforated Surface: A Self-Excited Large-Scale Instability. Physics of Fluids, 14(12), 4444-4447.

Feng, L. (2012). Tone-Like Signal in the Wind-Induced Noise of Perforated Plates. Acta Acustica United With Acustica, 98, 188-194.

Fluke. (2017). Fluke 922 Airflow Meter. (Fluke Corporation) Retrieved September 22, 2017, from http://www.fluke.com/fluke/m3en/HVAC-IAQ-Tools/AirTesters/Fluke-922.htm?PID=56154

Freymuth, P. (1966). On transition in a separated laminar boundary layer. Journal of Fluid Mechanics, 25(4), 683-704.

G.R.A.S. (n.d.). Calibration Chart 1/4" Pressure Microphone Type 40BP. Holte, Denmark: G.R.A.S. Sound \& Vibration.

Heller, H. H., \& Franken, P. A. (1988). Chapter 16: Noise of Gas Flow. In Noise and Vibration Control (pp. 515-524). Washington.

Howe, M. S. (1981). On the Theory of Unsteady Shearing Flow Over a Slot. Philosophical Transactions of the Royal Society of London. Series A, Mathematical and Physical Sciences, 303, 151-180.

Howe, M. S. (1981). The Influence of Mean Shear on Unsteady Aperture Flow, with Application to Acoustical Diffraction and Self-Sustained Cavity Oscillations. Journal of Fluid Mechanics, 109, 125-146.

ISO. (2003). ISO 1996-1: Acoustics - Description, Measurement, and Assessment of Environmental Noise - Part 1: Basic Quantities and Assessment Procedures. International Organization for Standardization. 
Kline, S. J., \& McClintock, F. A. (1953). Describing Uncertainties in Single-Sample Experiments. Mechanical Engineering, 73(1), 3-8.

Knisely, C., \& Rockwell, D. (1982). Self-sustained low-frequency components in an impinging shear layer. Journal of Fluid Mechanics, 116, 157-186.

Michalke, A. (1964). On the inviscid instability of the hyperbolic-tangent velocity profile. Journal of Fluid Mechanics, 19(4), 543-556.

Michalke, A. (1965). On spatially growing disturbances in an inviscid shear layer. Journal of Fluid Mechanics, 23(3), 521-544.

Michalke, A. (1972). The Instability of Free Shear Layers. Progress in Aerospace Sciences(12), 213-239.

Naudascher, E. (1967). From Flow Instability to Flow-Induced Excitation. Journal of the Hydraulics Division, 93(4), 15-40.

Nelson, P. A. (1982). Noise Generated by Flow Over Perforated Surfaces. Journal of Sound and Vibration, 83(1), 11-26.

Rayleigh, L. (1880). On the stability, or instability, of certain fluid motions. Proceedings of the London Mathematical Society, 11(1), 57-70.

Rockwell, D., \& Naudascher, E. (1979). Self-Sustained Oscillations of Impinging Free Shear Layers. Annual Review of Fluid Mechanics, 11, 67-94.

Schafer. (2017). Balcony Balustrades in Perforated Metal. (Schafer Perforated Metal) Retrieved April 28, 2017, from http://www.schaeferlochbleche.de/en/infocenter/perforated-metal-applications/perforated-metalbalcony-balustrades/

Tollmien, W. (1935). General instability criterion of laminar velocity distributions.

Tsui, C. Y., \& Flandro, G. A. (1977). Self-Induced Sound Generation by Flow Over Perforated Duct Liners. Journal of Sound and Vibration, 50(3), 315-331.

Vollmers, H. (2001). Detection of Vortices and Quantitative Evaluation of their Main Parameters from Experimental Velocity Data. Measurement Science and Technology, 12, 1199-1207. 
Wellington. (2017). Stock Mild Steel. (Wellington Perforated Sheet \& Plate Inc.) Retrieved 08 23, 2017, from http://wellingtonperforated.ca/plain-steel/stock-mildsteel/products.html

Ziada, S., \& Shine, S. (1999). Strouhal Numbers of Flow-Excited Acoustic Resonance of Closed Side Branches. Journal of Fluids and Structures(13), 127-142. 


\section{APPENDIX A UNCERTAINTY ANALYSIS}

\section{A.1. Introduction}

In this appendix, the errors of various measured and calculated values are evaluated. The method proposed by Kline and McClintock is used (Kline \& McClintock, 1953). The uncertainty of a dependent variable $Y$ which is a function of a set of variables, $X_{i}$, is calculated as follows:

$$
d Y=\sqrt{\sum_{i=1}^{n}\left(\frac{\partial Y}{\partial X_{i}} d X_{i}\right)^{2}}
$$

Therefore, the absolute uncertainty of $Y$ is a function of the uncertainty of each individual variable, $X_{i}$, and the derivative of $Y$ with respect to each variable.

\section{A.2. Mean Flow Velocity}

The mean flow velocity from the wind tunnel is found using a pitot tube positioned in the center of the wind tunnel exit section and a Fluke 922 airflow meter. A linear relationship is developed between the blower motor frequency and the mean exit velocity, which is $0.7167(\mathrm{~m} / \mathrm{s}) / \mathrm{Hz}$.

The Fluke 922 airflow meter has a resolution of $0.001 \mathrm{~m} / \mathrm{s}$ and a manufacturer's quoted accuracy of $\pm 2.5 \%$ at a reading of $10.00 \mathrm{~m} / \mathrm{s}$ (Fluke, 2017). This corresponds to an 
absolute uncertainty of $0.25 \mathrm{~m} / \mathrm{s}$. The resolution of the blower motor frequency is $0.1 \mathrm{~Hz}$. Therefore, the value of the frequency is accurate to $\pm 0.05 \mathrm{~Hz}$, which corresponds to \pm $0.035835 \mathrm{~m} / \mathrm{s}$. At a mean flow velocity of $10 \mathrm{~m} / \mathrm{s}$, this gives a percent error of $\pm 0.72 \%$. In this case, equation (15) reduces to:

$$
\begin{aligned}
& d Y=\sqrt{d X_{1}{ }^{2}+d X_{2}{ }^{2}} \\
& d V=\sqrt{(0.25 \mathrm{~m} / \mathrm{s})^{2}+(0.035835 \mathrm{~m} / \mathrm{s})^{2}} \\
& d V=0.253 \mathrm{~m} / \mathrm{s}
\end{aligned}
$$

This corresponds to a percentage error for the mean flow velocity of $2.53 \%$ at a mean flow velocity of $10 \mathrm{~m} / \mathrm{s}$. The percentage error decreases at higher flow velocities.

\section{A.3. Acoustic Measurements}

Acoustic measurements are taken with a G.R.A.S. 40BP 1/4-inch pressure microphone. The manufacturer's quoted uncertainty for this microphone is $\pm 0.06 \mathrm{~dB}$ (G.R.A.S.). The error in the microphone measurements can be expressed as follows, where $S P L_{a}$ is the actual sound pressure level and $S P L_{m}$ is the measured sound pressure level:

$$
\begin{aligned}
& S P L_{m}-S P L_{a}=0.06 \\
& 20 \log \frac{P_{m}}{P_{\text {ref }}}-20 \log \frac{P_{a}}{P_{\text {ref }}}=0.06 \\
& 20 \log \frac{P_{m}}{P_{a}}=0.06 \\
& \frac{P_{m}}{P_{a}}=10^{\frac{0.06}{20}}
\end{aligned}
$$




$$
\frac{P_{m}}{P_{a}}=1.0069
$$

Therefore, the percentage error of the microphone measurements is $\pm 0.69 \%$.

\section{A.4. Calculated Values}

The uncertainty of the calculated values is found using the propagation of uncertainties and equation (15). The Strouhal number is calculated using the following equation:

$$
\begin{aligned}
& S t=\frac{f * d}{V} \\
& S t=\frac{f *\left(\frac{4}{\pi}\right) * H}{V_{\text {mean }}}
\end{aligned}
$$

The frequency comes from the microphone measurements, and has a resolution of $1 \mathrm{~Hz}$. The lowest frequency of the measured tones is $387 \mathrm{~Hz}(\mathrm{St}=0.625)$, for the case of a $12.7 \mathrm{~mm}$ gap width and a $10 \mathrm{~m} / \mathrm{s}$ flow velocity. This corresponds to an uncertainty of \pm $0.26 \%$ at this frequency. The characteristic dimension, $d$, is defined as the equivalent diameter, $d_{e}=\left(\frac{4}{\pi}\right) H$. The gap width, $H$, is measured with a digital caliper with a resolution of 0.0005 inches $(0.0127 \mathrm{~mm})$. For the smallest gap width, $H=0.125$ inches $(3.175 \mathrm{~mm})$, this corresponds to an uncertainty of $\pm 0.40 \%$. As previously stated, the uncertainty of the mean flow velocity is $\pm 2.53 \%$. Therefore, using equation (15), the uncertainty of the Strouhal number calculation is as follows: 


$$
\begin{aligned}
& d(S t)=\sqrt{\left(\frac{\partial S t}{\partial f} d f\right)^{2}+\left(\frac{\partial S t}{\partial H} d H\right)^{2}+\left(\frac{\partial S t}{\partial V} d V\right)^{2}} \\
& d(S t)=\sqrt{\left(\frac{\left(\frac{4}{\pi}\right) * H}{V} d f\right)^{2}+\left(\frac{f *\left(\frac{4}{\pi}\right)}{V} d H\right)^{2}+\left(-\frac{f *\left(\frac{4}{\pi}\right) * H}{V^{2}} d V\right)^{2}}
\end{aligned}
$$

For a conservative estimate, the following case is used: $H=12.7 \mathrm{~mm}, V=10 \mathrm{~m} / \mathrm{s}, f$ $=387 \mathrm{~Hz}, \mathrm{St}=0.625, d f=1 \mathrm{~Hz}, d H=0.0127 \mathrm{~mm}, d V=0.253 \mathrm{~m} / \mathrm{s}$. This gives an absolute uncertainty for the Strouhal number of \pm 0.01593 , or a relative uncertainty of $\pm 2.55 \%$.

The normalized sound pressure, $P^{*}$, is calculated using the following equation:

$$
P^{*}=\frac{P}{\frac{1}{2} \rho * V_{\text {mean }}^{2}}
$$

The sound pressure, $P$, has an uncertainty of $\pm 0.69 \%$, and the mean velocity has an uncertainty of $\pm 2.53 \%$, as previously stated. The density of air at $20^{\circ} \mathrm{C}, \rho=1.204 \mathrm{~kg} / \mathrm{m}^{3}$, is used for all calculations of $P^{*}$. The actual air temperature may vary by up to $\pm 5^{\circ} \mathrm{C}$. The air density at $25^{\circ} \mathrm{C}$ is $1.184 \mathrm{~kg} / \mathrm{m}^{3}$, which is a difference of $\pm 0.020 \mathrm{~kg} / \mathrm{m}^{3}$, or $\pm 1.66 \%$. Using equation (15), the uncertainty of the normalized sound pressure calculation is as follows:

$$
\begin{aligned}
& d P^{*}=\sqrt{\left(\frac{\partial P^{*}}{\partial P} d P\right)^{2}+\left(\frac{\partial P^{*}}{\partial \rho} d \rho\right)^{2}+\left(\frac{\partial P^{*}}{\partial V} d V\right)^{2}} \\
& d P^{*}=\sqrt{\left(\frac{1}{\frac{1}{2} \rho V^{2}} d P\right)^{2}+\left(-\frac{P}{\frac{1}{2} \rho^{2} V^{2}} d \rho\right)^{2}+\left(-2 * \frac{P}{\frac{1}{2} \rho V^{3}} d V\right)^{2}}
\end{aligned}
$$


The largest percentage uncertainty exists for low velocities and low sound pressures. Therefore, for a conservative estimate, the following values are used: $P=0.05$ $P a, V=10 \mathrm{~m} / \mathrm{s}, \rho=1.204 \mathrm{~kg} / \mathrm{m}^{3}, P^{*}=8.306 \times 10^{-4}, d P=3.466 \times 10^{-4} \mathrm{~Pa}, d V=0.253 \mathrm{~m} / \mathrm{s}$, $d \rho=0.020 \mathrm{~kg} / \mathrm{m}^{3}$. This gives an absolute uncertainty for the normalized sound pressure of $\pm 4.46 \times 10^{-5}$, or a relative uncertainty of $\pm 5.37 \%$.

\section{A.5. Particle Image Velocimetry Measurements}

The quality of the PIV images was verified by checking the vector validation rate, calculated by the Insight $4 \mathrm{G}$ software. This gives a percentage of the velocity vectors in an image which meet a specified set of standards and are considered "good" vectors. For the PIV results shown in this thesis, the vector validation rate was consistently 98 to $99 \%$. The error in the PIV images is further reduced by taking the average of 200 images at each setpoint.

The pixel size for the PIV images is in the range of 15 to $16 \mu m$, with an interrogation region of $24-b y-24$ pixels. Therefore, each interrogation region is 0.360 to $0.384 \mathrm{~mm}$. The PIV images are only spatially accurate to within half an interrogation region, or to within 0.180 to $0.192 \mathrm{~mm}$.

The phase-locked PIV images were obtained by applying a time delay to the trigger to take images at a specific point in the acoustic cycle. The resolution of this time delay is $0.01 \mathrm{~ms}$, so the point at which images are taken is $\pm 0.005 \mathrm{~ms}$ from the true value. The highest frequency phase-locking is done at a frequency of $4530 \mathrm{~Hz}$ for a flow velocity of $30 \mathrm{~m} / \mathrm{s}$. The period of this oscillation is $0.22075 \mathrm{~ms}$. The percentage error of the image 
capture time for this case is $\pm 2.265 \%$ of a cycle, or $\pm 8.154^{\circ}$. This error decreases at lower phase-locking frequencies. 


\section{APPENDIX B ADDITIONAL RESULTS}

\section{B.1. Acoustic Results - Perforated Plate}

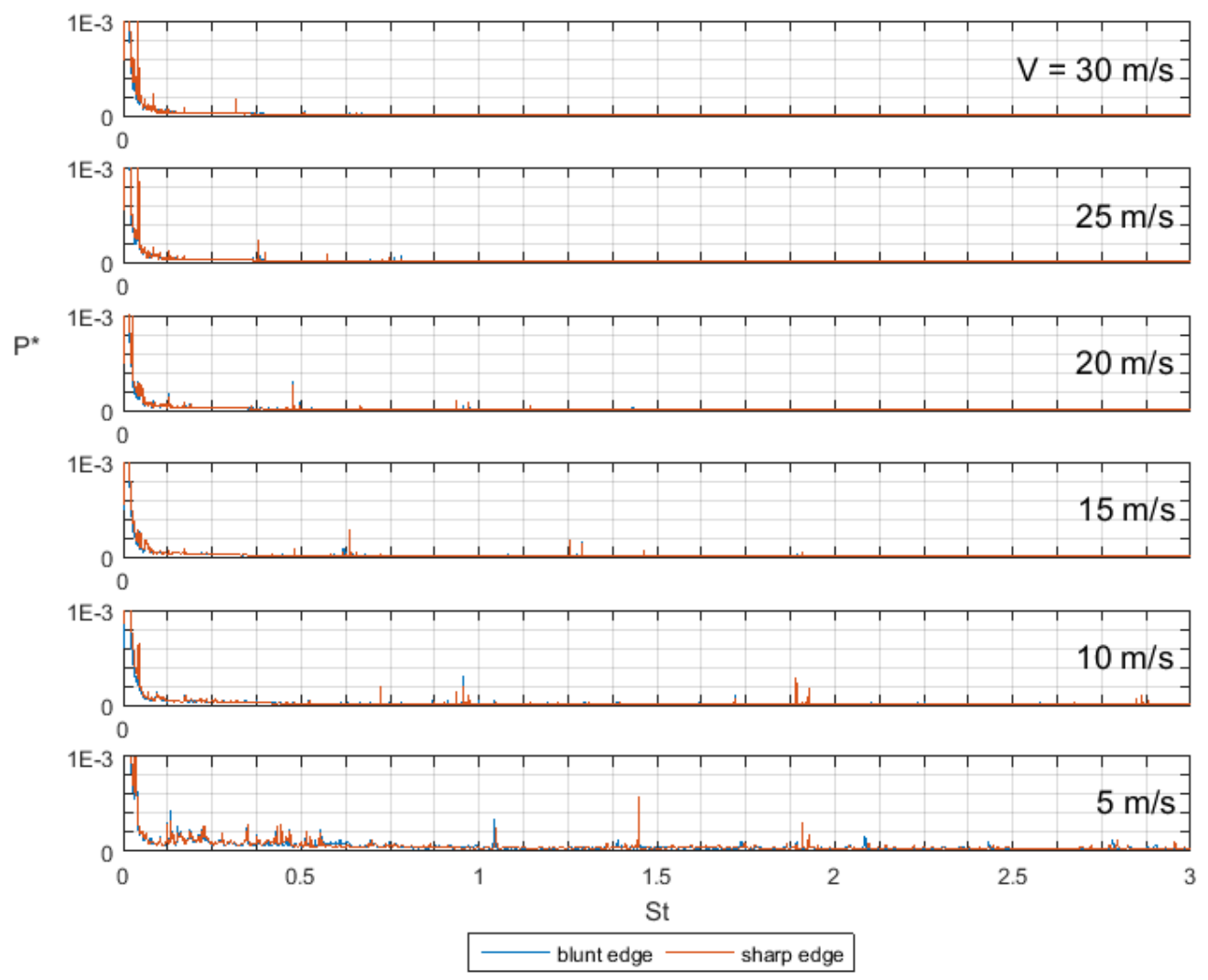

Figure 63: Frequency spectra for a perforated plate with a hole diameter of $6.35 \mathrm{~mm}$, an angle of incidence of 0 degrees, and a flow velocity of 5 to $30 \mathrm{~m} / \mathrm{s}$. The red line is for the sharp edge of the plate exposed to the flow, while the blue line is for the blunt edge exposed to the flow. 


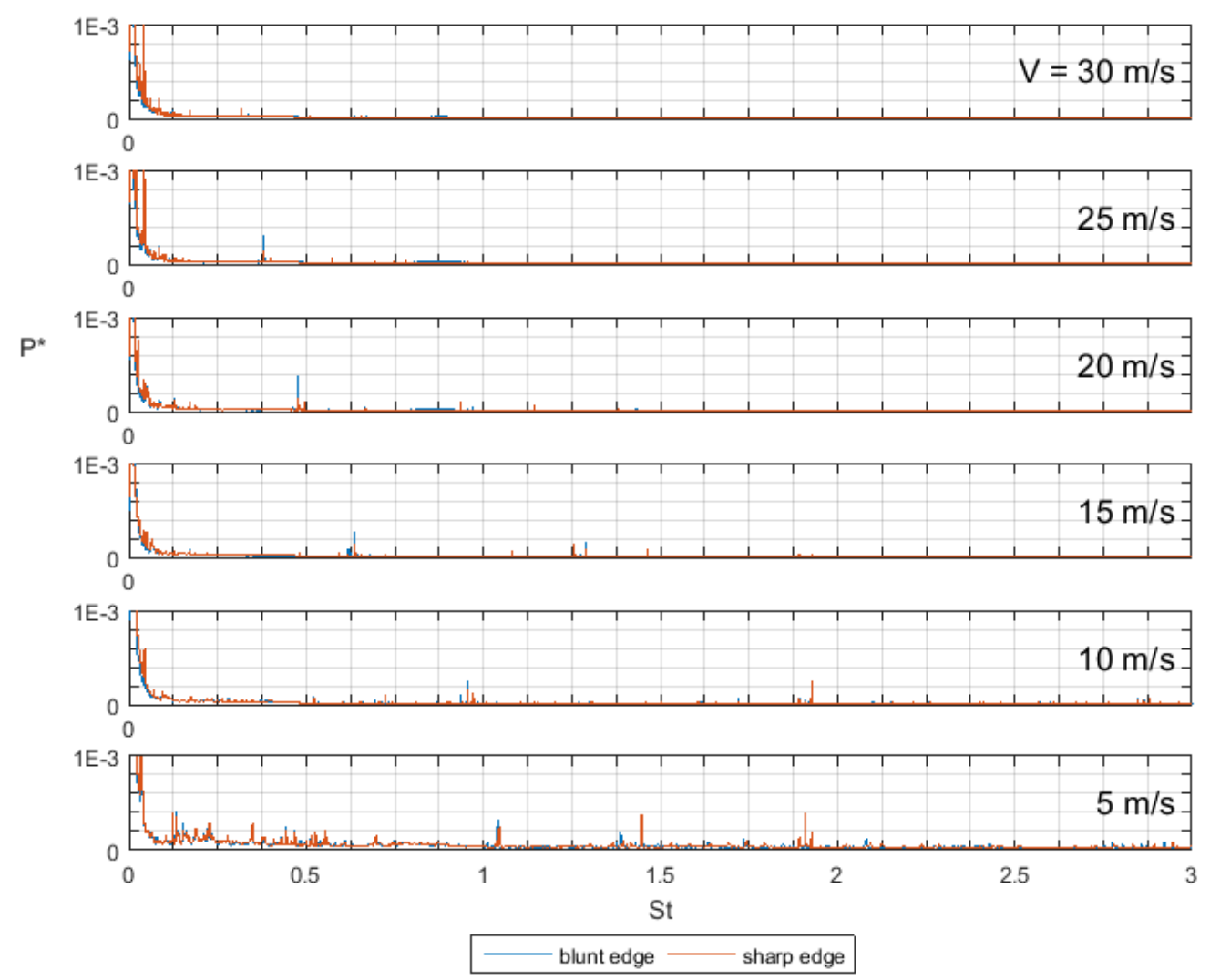

Figure 64: Frequency spectra for a perforated plate with a hole diameter of $6.35 \mathrm{~mm}$, an angle of incidence of 5 degrees, and a flow velocity of 5 to $30 \mathrm{~m} / \mathrm{s}$. The red line is for the sharp edge of the plate exposed to the flow, while the blue line is for the blunt edge exposed to the flow. 


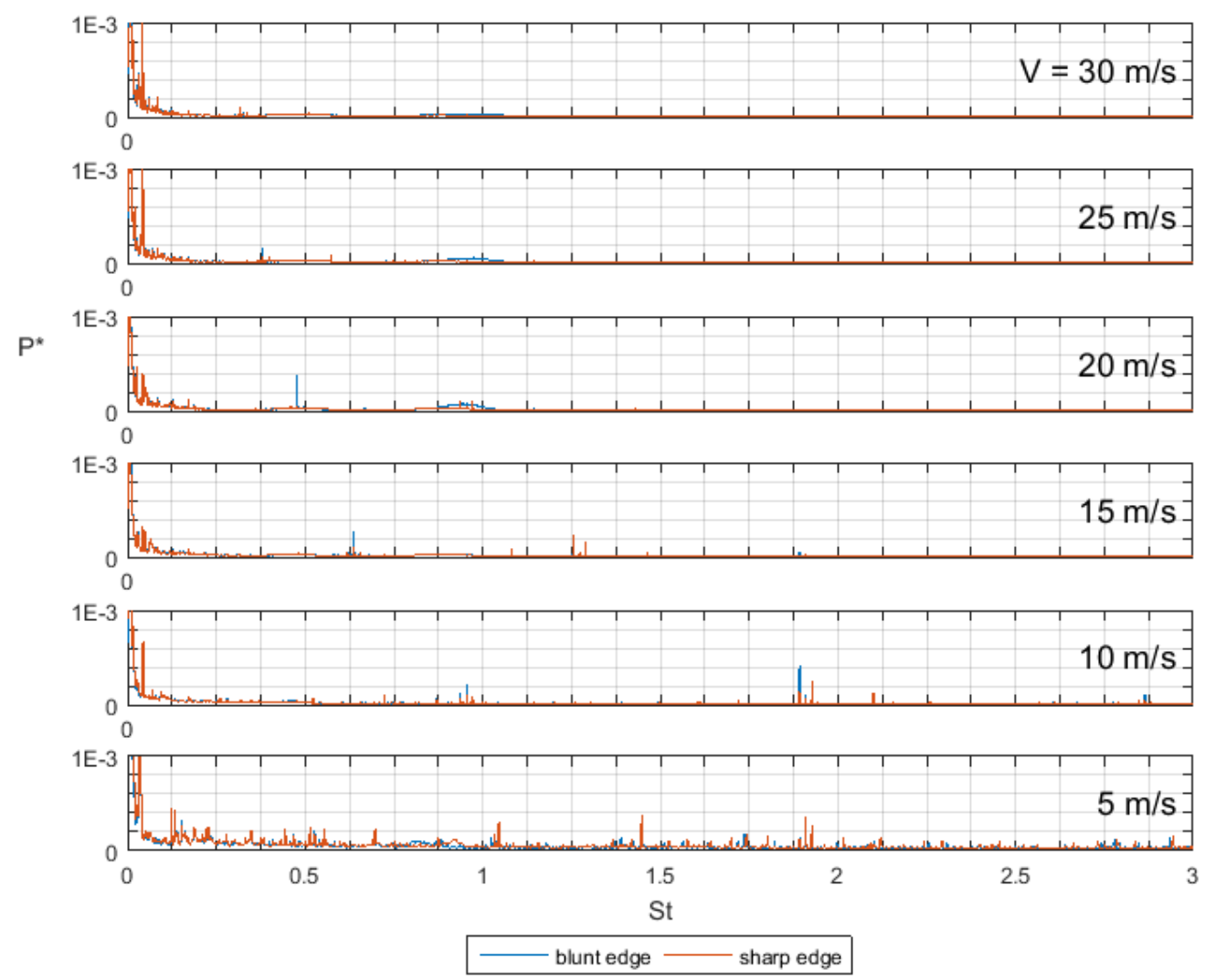

Figure 65: Frequency spectra for a perforated plate with a hole diameter of $6.35 \mathrm{~mm}$, an angle of incidence of 10 degrees, and a flow velocity of 5 to $30 \mathrm{~m} / \mathrm{s}$. The red line is for the sharp edge of the plate exposed to the flow, while the blue line is for the blunt edge exposed to the flow. 


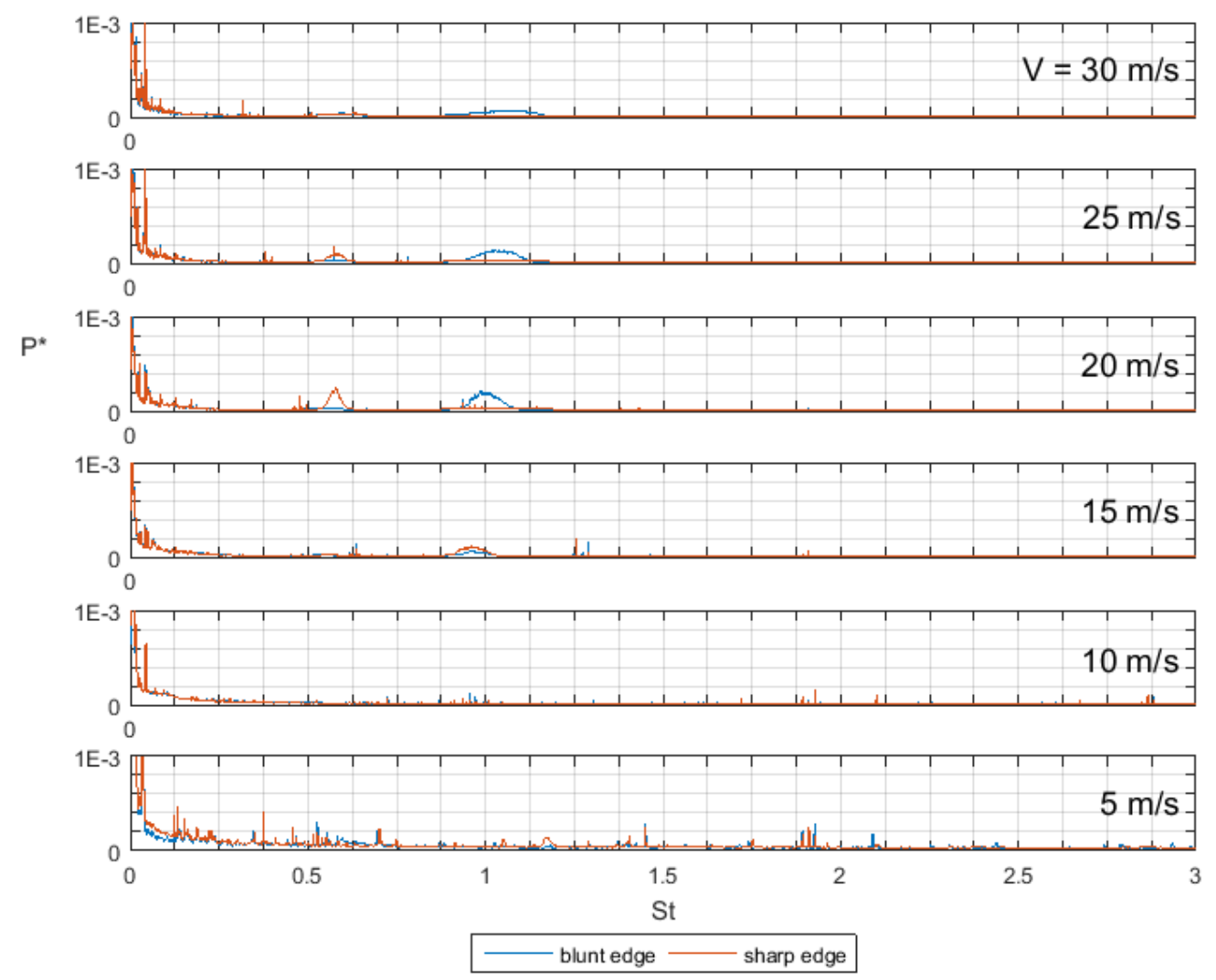

Figure 66: Frequency spectra for a perforated plate with a hole diameter of $6.35 \mathrm{~mm}$, an angle of incidence of 15 degrees, and a flow velocity of 5 to $30 \mathrm{~m} / \mathrm{s}$. The red line is for the sharp edge of the plate exposed to the flow, while the blue line is for the blunt edge exposed to the flow. 


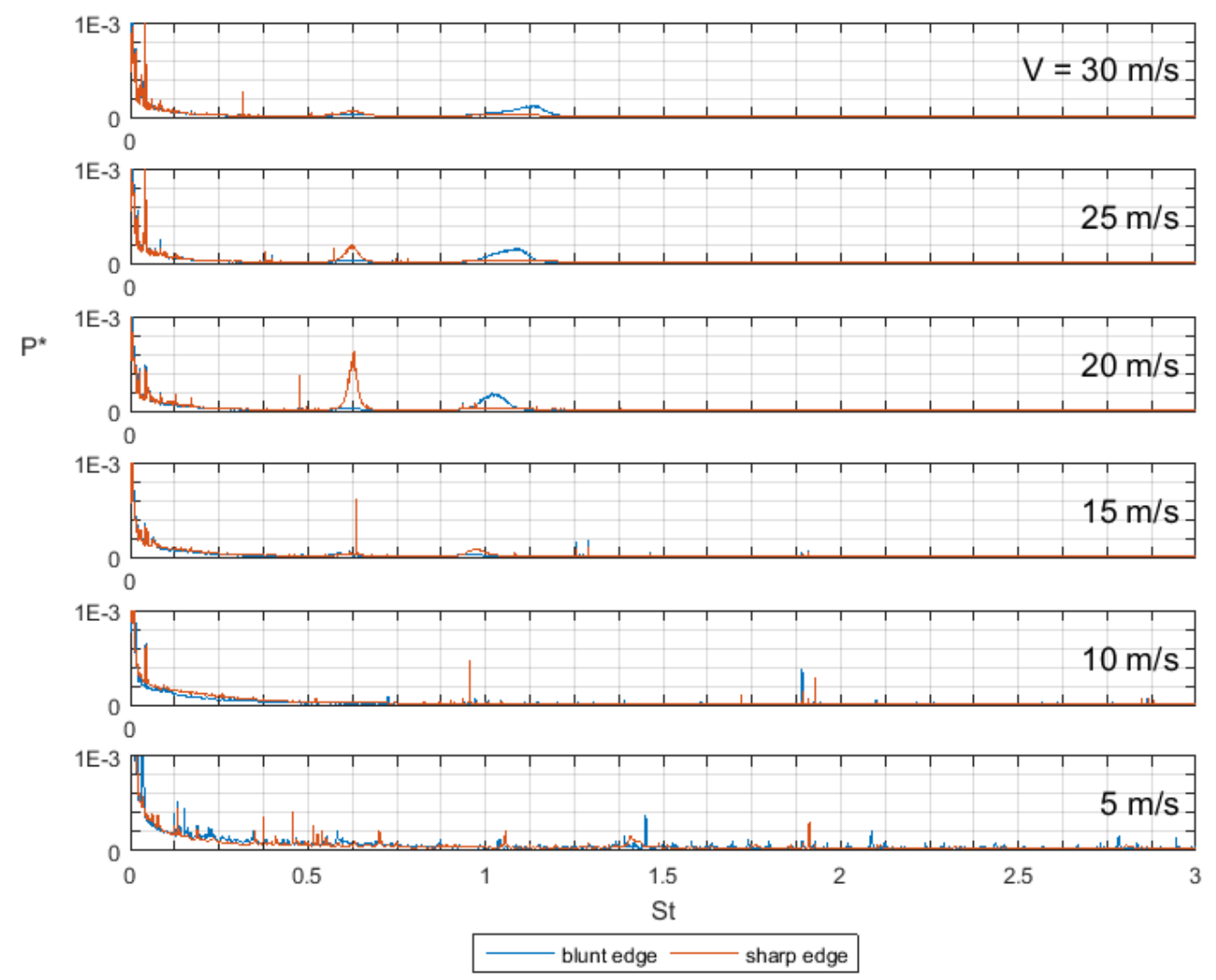

Figure 67: Frequency spectra for a perforated plate with a hole diameter of $6.35 \mathrm{~mm}$, an angle of incidence of 20 degrees, and a flow velocity of 5 to $30 \mathrm{~m} / \mathrm{s}$. The red line is for the sharp edge of the plate exposed to the flow, while the blue line is for the blunt edge exposed to the flow. 


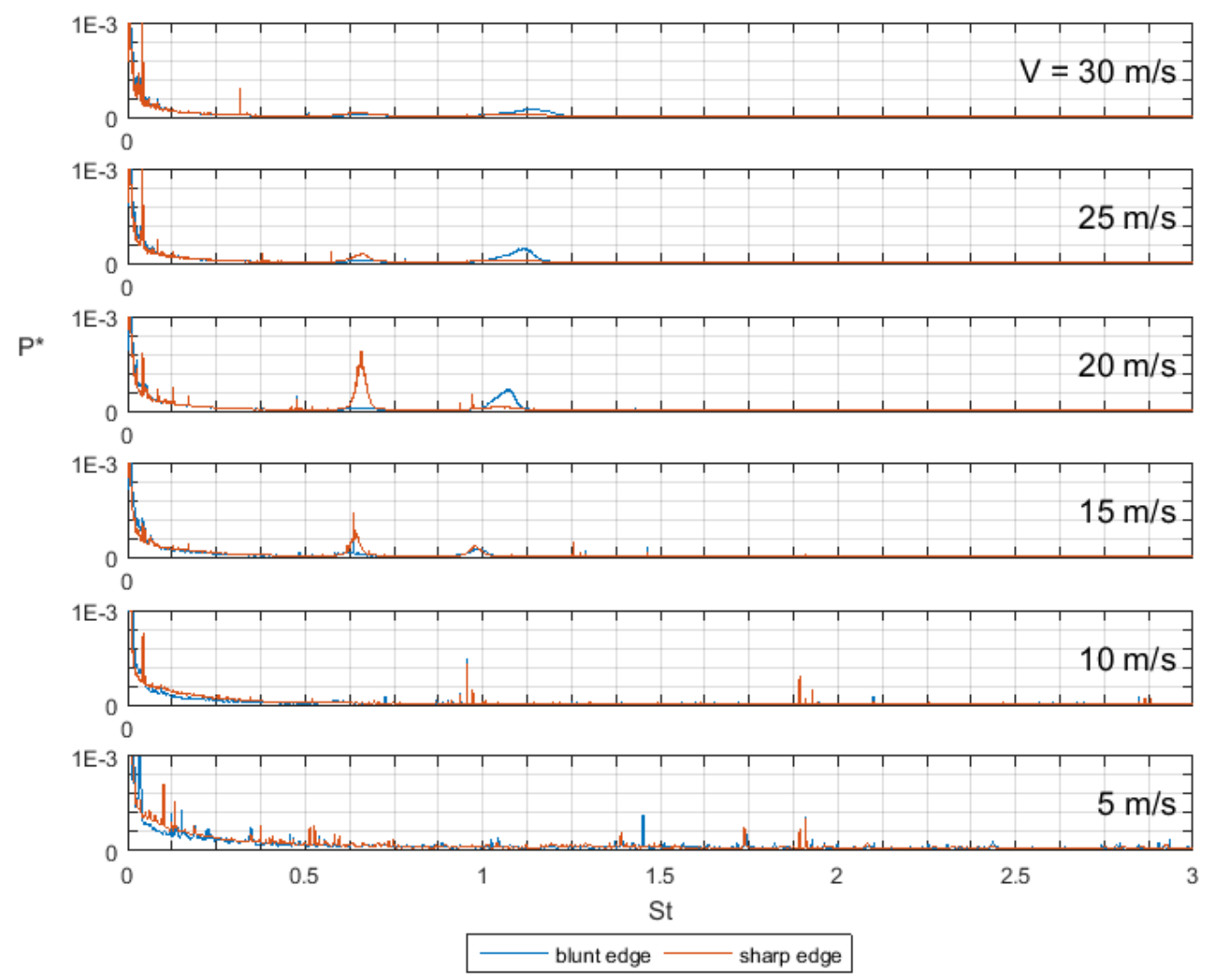

Figure 68: Frequency spectra for a perforated plate with a hole diameter of $6.35 \mathrm{~mm}$, an angle of incidence of 25 degrees, and a flow velocity of 5 to $30 \mathrm{~m} / \mathrm{s}$. The red line is for the sharp edge of the plate exposed to the flow, while the blue line is for the blunt edge exposed to the flow. 


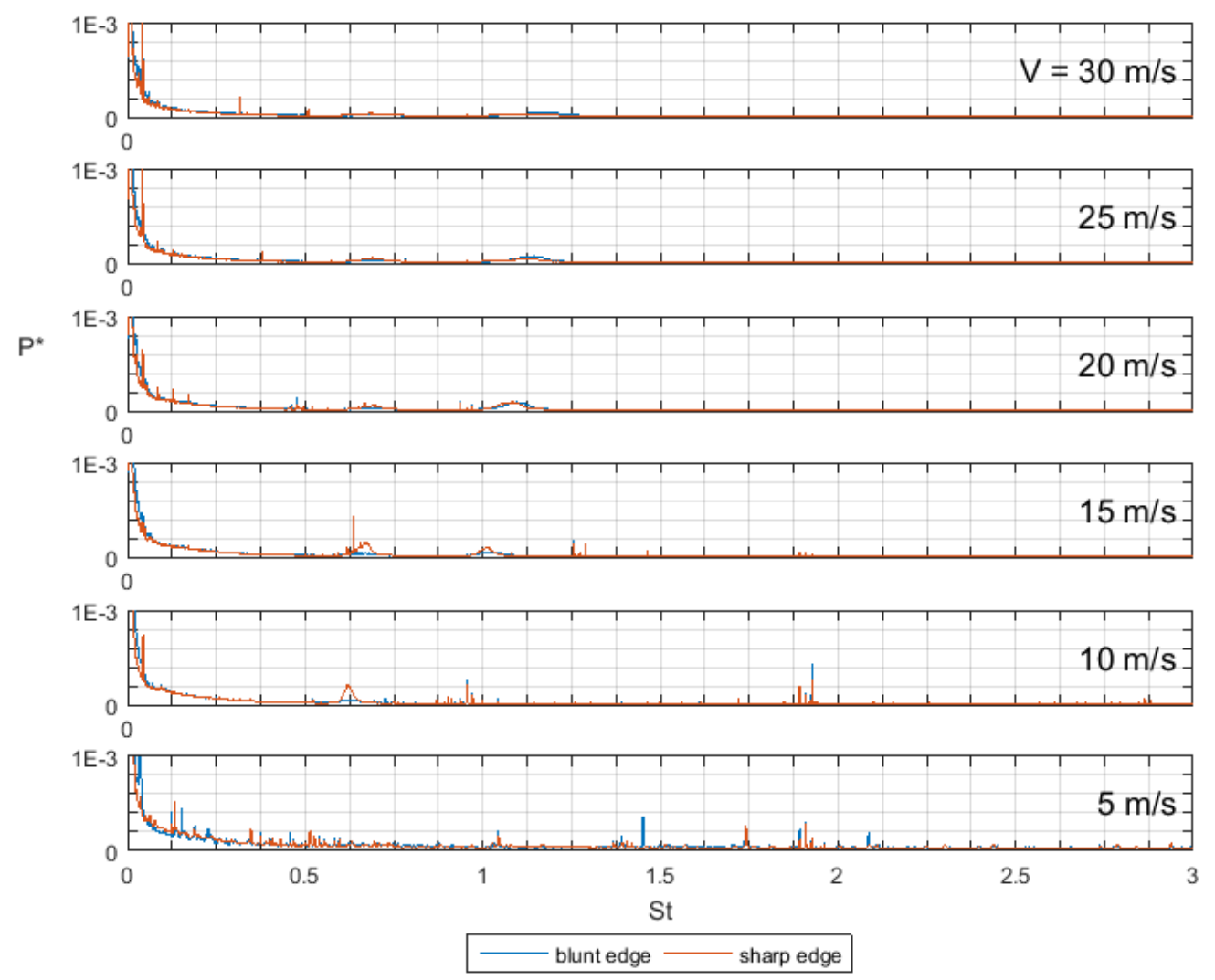

Figure 69: Frequency spectra for a perforated plate with a hole diameter of $6.35 \mathrm{~mm}$, an angle of incidence of 30 degrees, and a flow velocity of 5 to $30 \mathrm{~m} / \mathrm{s}$. The red line is for the sharp edge of the plate exposed to the flow, while the blue line is for the blunt edge exposed to the flow. 


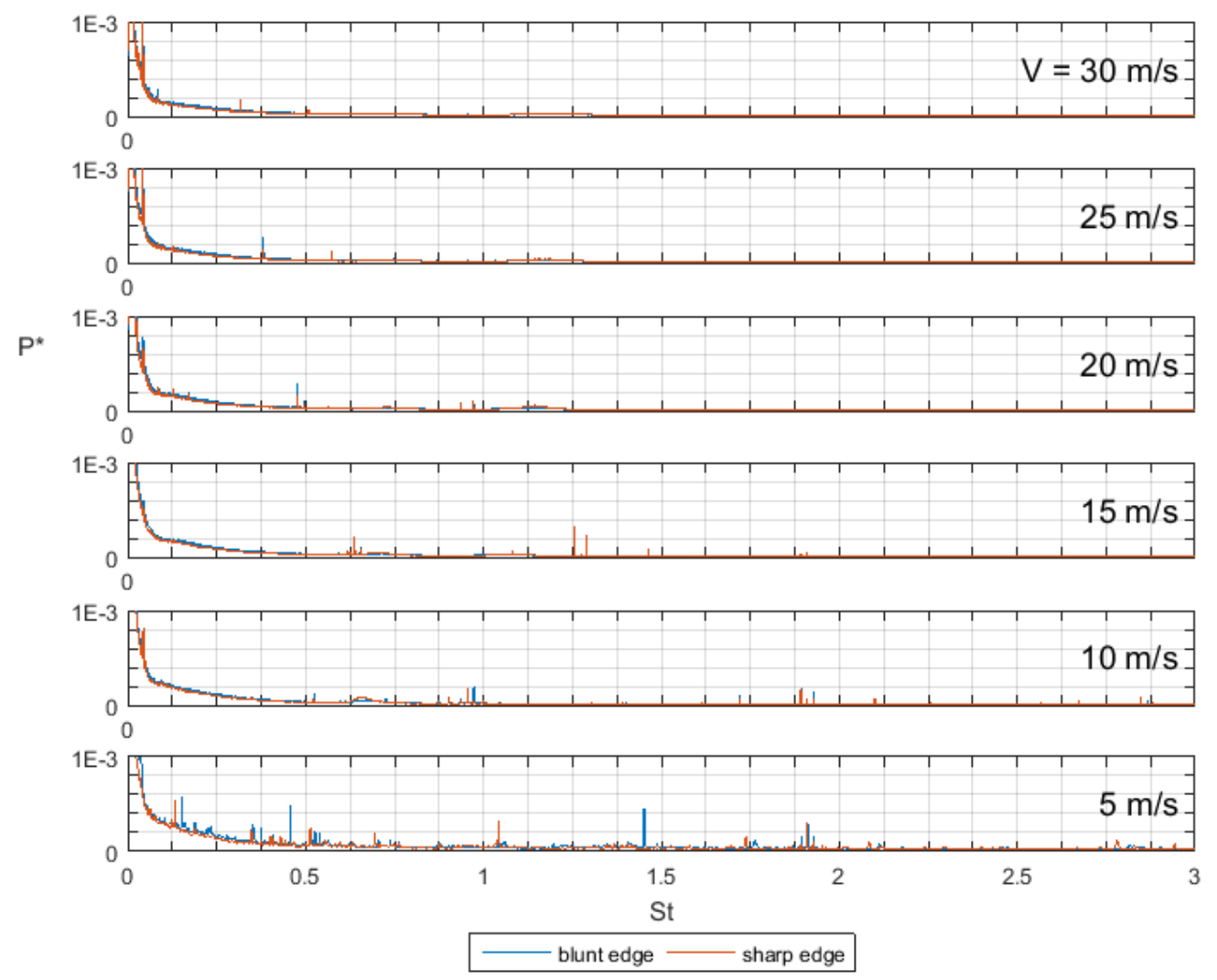

Figure 70: Frequency spectra for a perforated plate with a hole diameter of $6.35 \mathrm{~mm}$, an angle of incidence of 35 degrees, and a flow velocity of 5 to $30 \mathrm{~m} / \mathrm{s}$. The red line is for the sharp edge of the plate exposed to the flow, while the blue line is for the blunt edge exposed to the flow. 


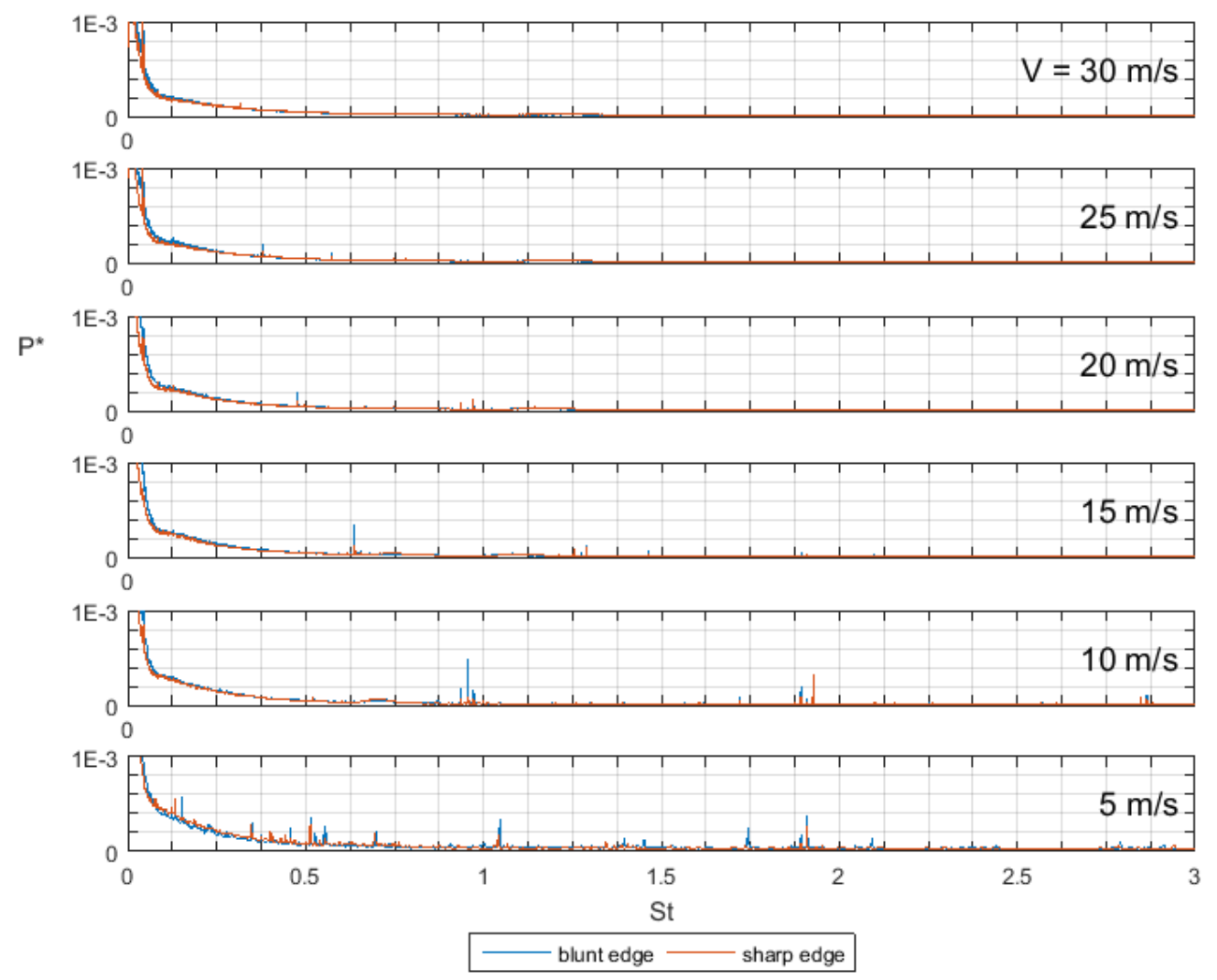

Figure 71: Frequency spectra for a perforated plate with a hole diameter of $6.35 \mathrm{~mm}$, an angle of incidence of 40 degrees, and a flow velocity of 5 to $30 \mathrm{~m} / \mathrm{s}$. The red line is for the sharp edge of the plate exposed to the flow, while the blue line is for the blunt edge exposed to the flow. 


\section{B.2. Acoustic Results - 2D Model}

\section{B.2.1. Effect of flow velocity, gap width $=3.175 \mathrm{~mm}$}

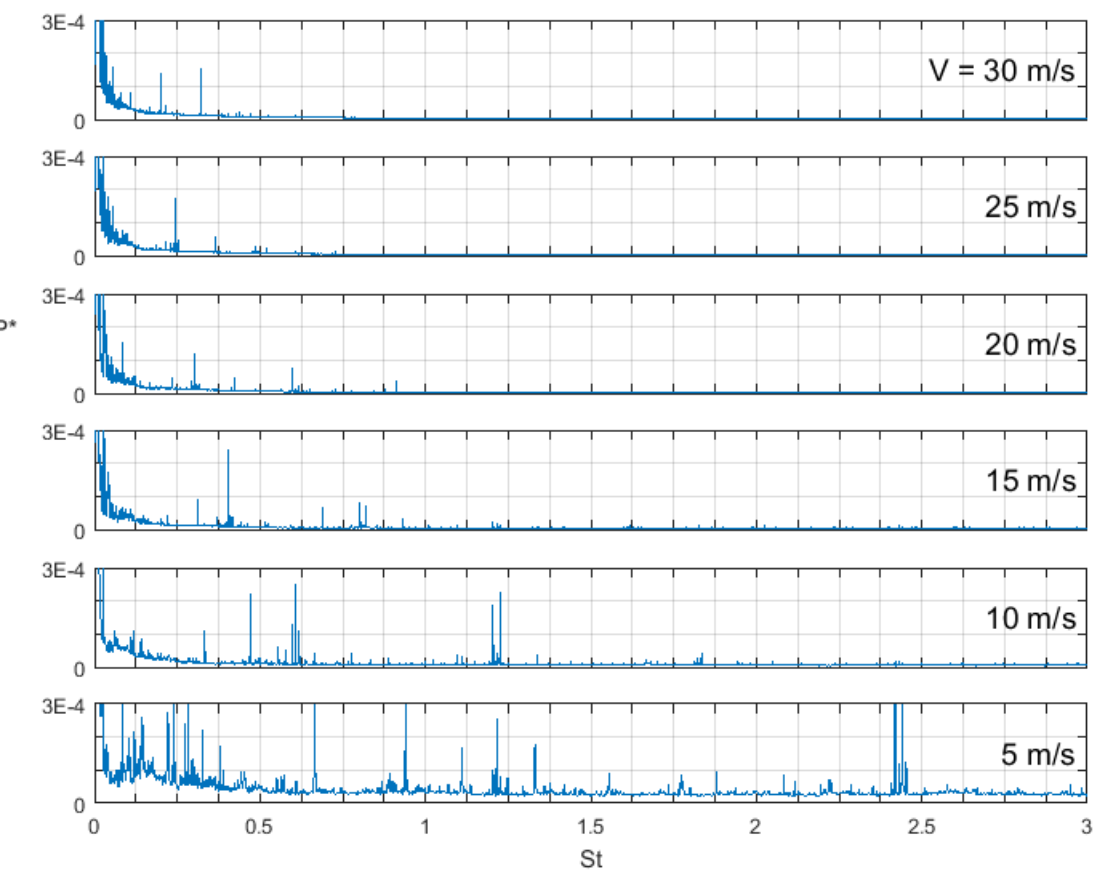

Figure 72: Frequency spectra for a gap width of $3.175 \mathrm{~mm}$, an angle of incidence of 0 degrees, and a flow velocity of 5 to $30 \mathrm{~m} / \mathrm{s}$. 


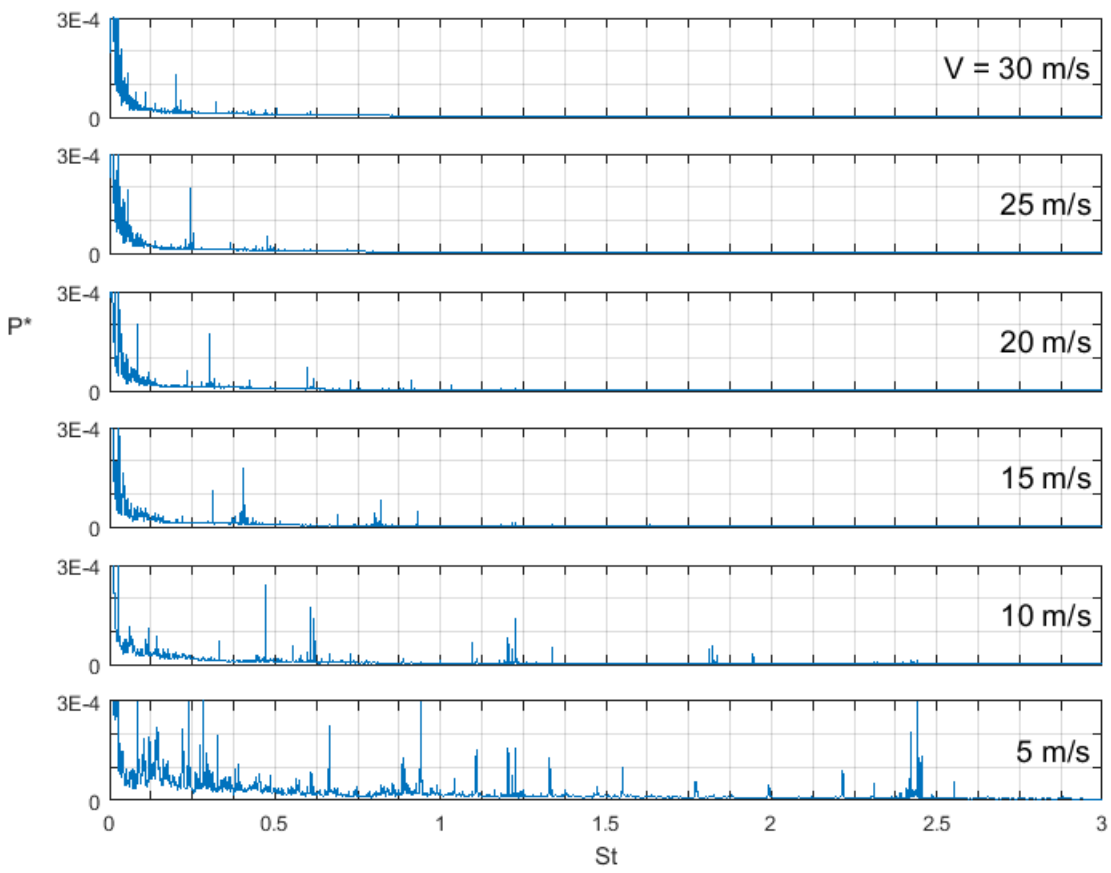

Figure 73: Frequency spectra for a gap width of $3.175 \mathrm{~mm}$, an angle of incidence of 5 degrees, and a flow velocity of 5 to $30 \mathrm{~m} / \mathrm{s}$.

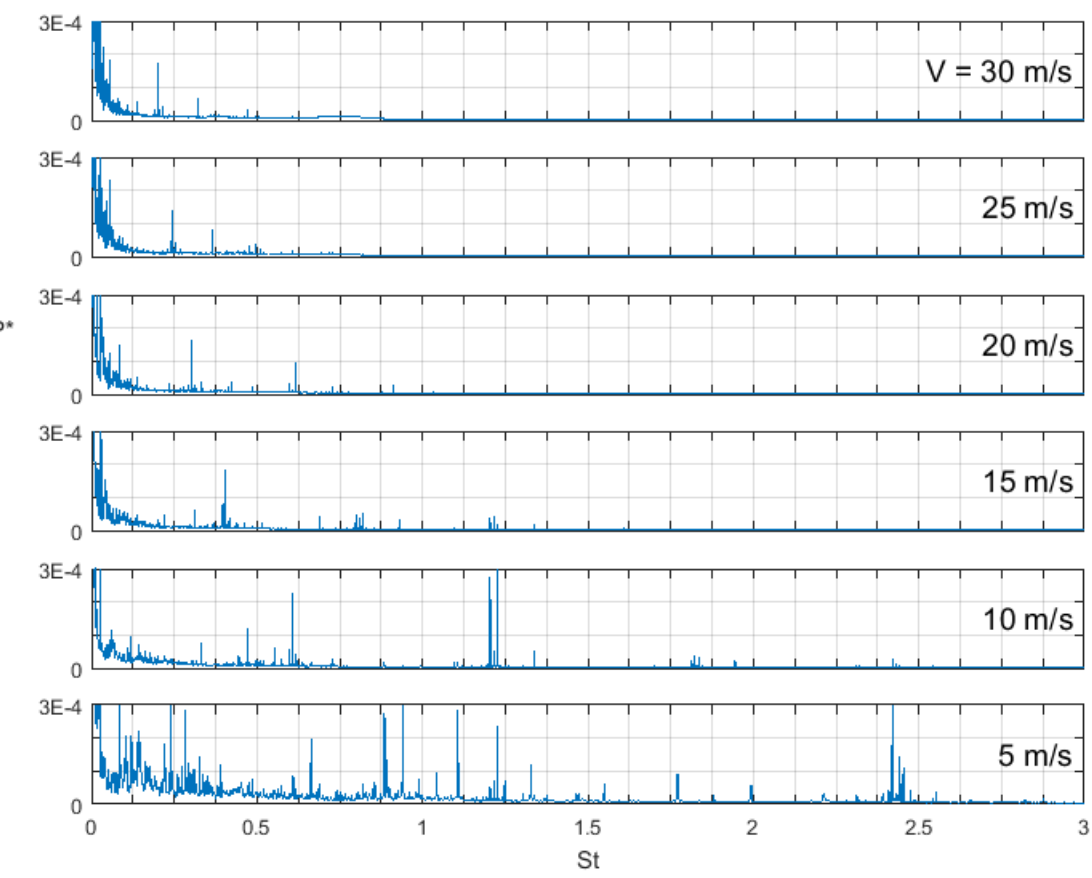

Figure 74: Frequency spectra for a gap width of $3.175 \mathrm{~mm}$, an angle of incidence of 10 degrees, and a flow velocity of 5 to $30 \mathrm{~m} / \mathrm{s}$. 


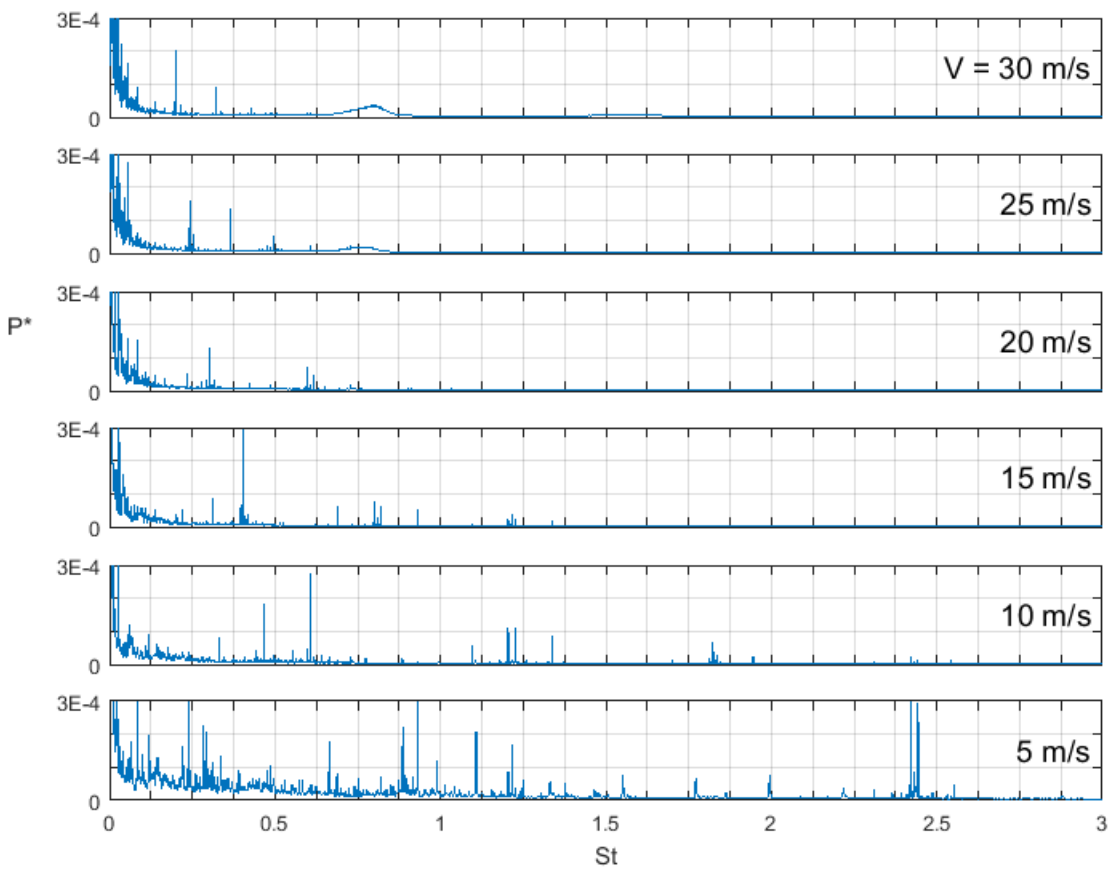

Figure 75: Frequency spectra for a gap width of $3.175 \mathrm{~mm}$, an angle of incidence of 15 degrees, and a flow velocity of 5 to $30 \mathrm{~m} / \mathrm{s}$.

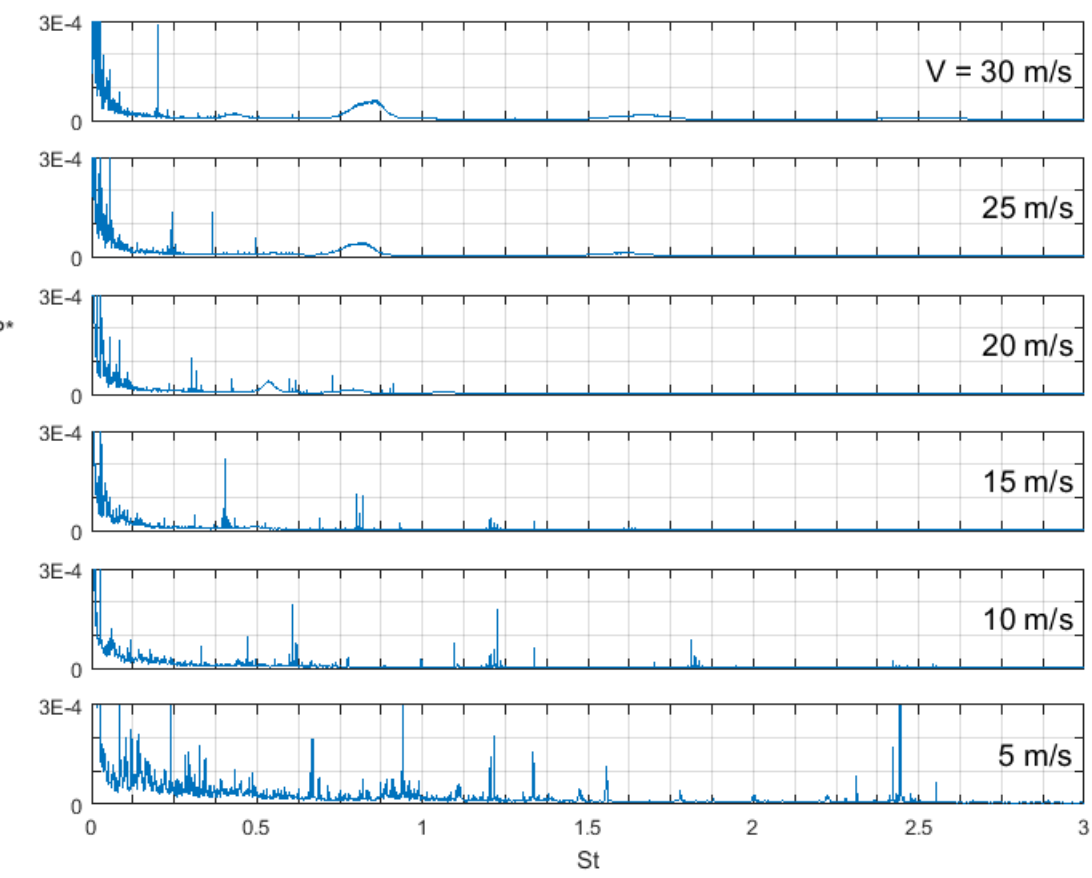

Figure 76: Frequency spectra for a gap width of $3.175 \mathrm{~mm}$, an angle of incidence of 20 degrees, and a flow velocity of 5 to $30 \mathrm{~m} / \mathrm{s}$. 


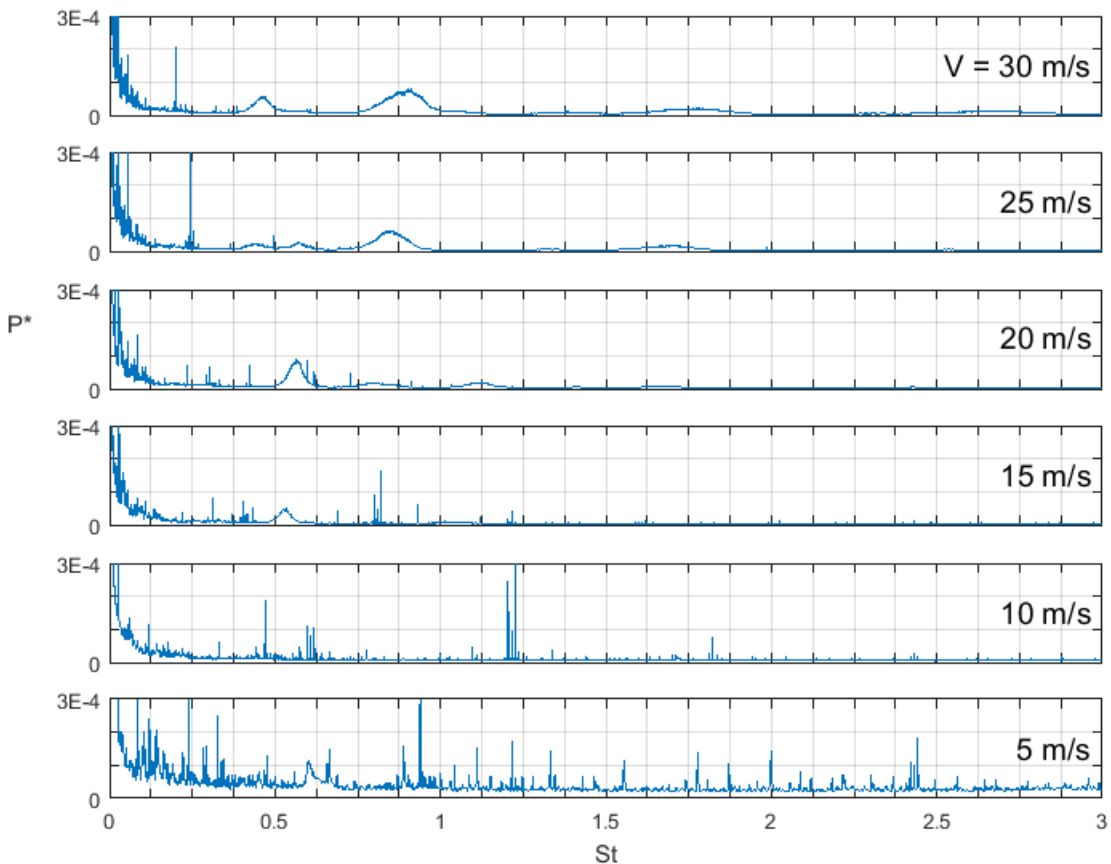

Figure 77: Frequency spectra for a gap width of $3.175 \mathrm{~mm}$, an angle of incidence of 25 degrees, and a flow velocity of 5 to $30 \mathrm{~m} / \mathrm{s}$.

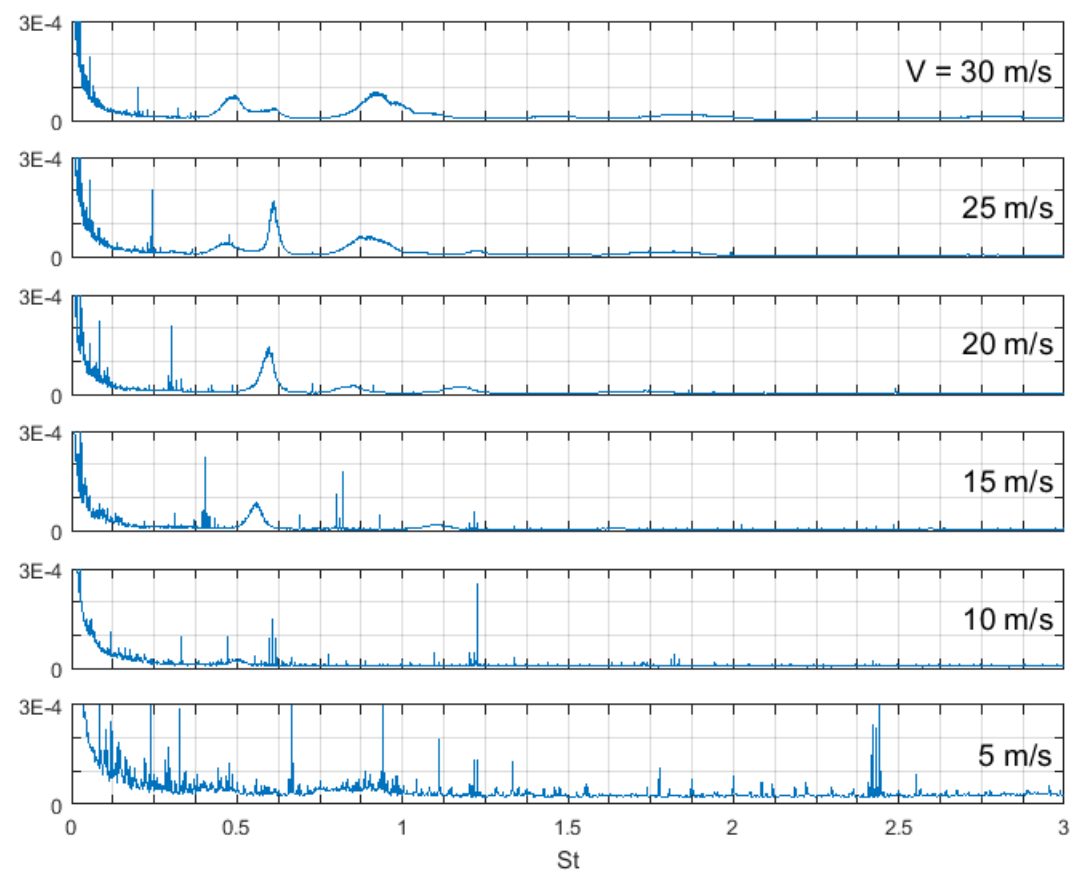

Figure 78: Frequency spectra for a gap width of $3.175 \mathrm{~mm}$, an angle of incidence of 30 degrees, and a flow velocity of 5 to $30 \mathrm{~m} / \mathrm{s}$. 


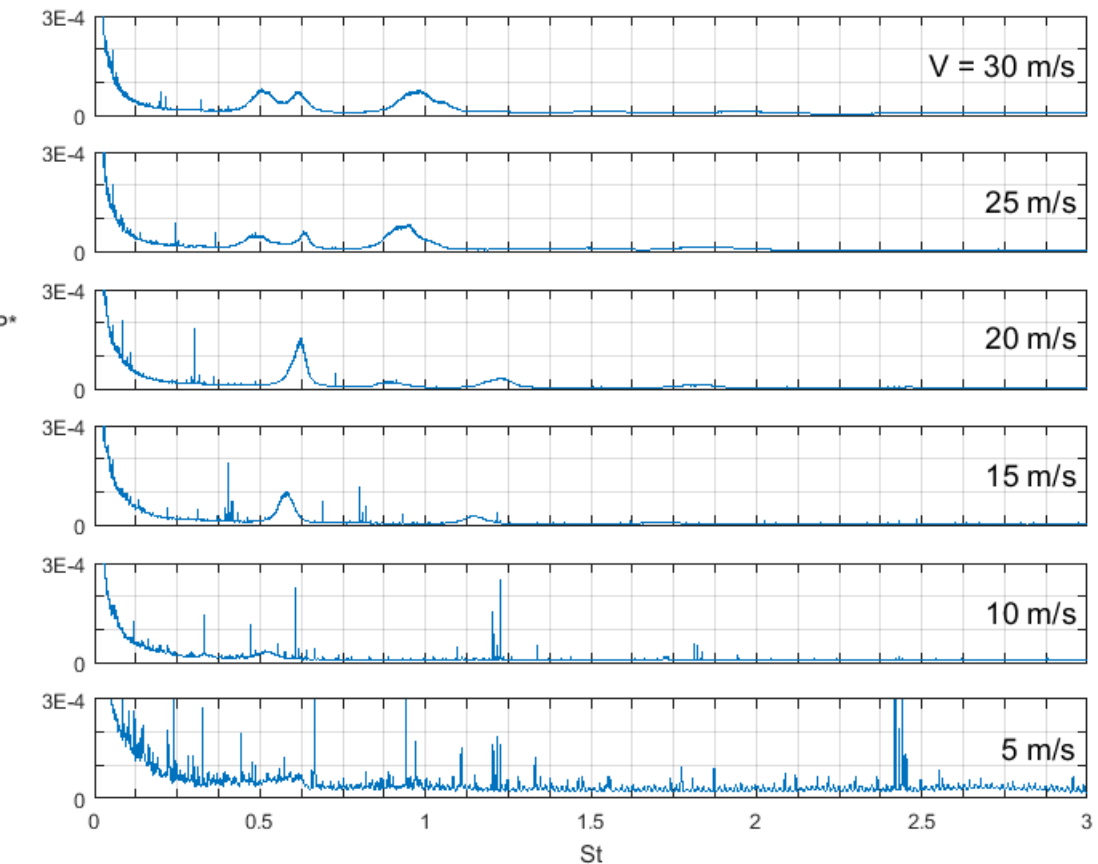

Figure 79: Frequency spectra for a gap width of $3.175 \mathrm{~mm}$, an angle of incidence of 35 degrees, and a flow velocity of 5 to $30 \mathrm{~m} / \mathrm{s}$.
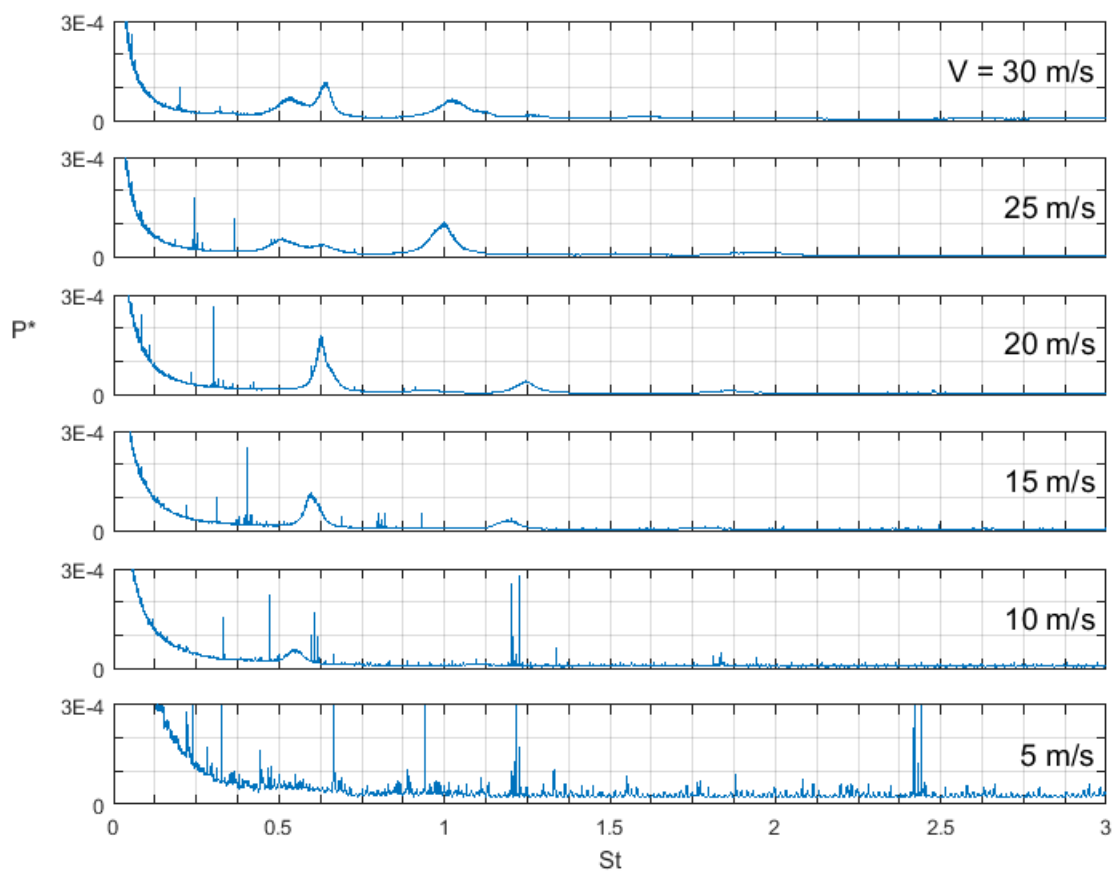

Figure 80: Frequency spectra for a gap width of $3.175 \mathrm{~mm}$, an angle of incidence of 40 degrees, and a flow velocity of 5 to $30 \mathrm{~m} / \mathrm{s}$. 


\section{B.2.2. Effect of flow velocity, gap width $=6.35 \mathrm{~mm}$}

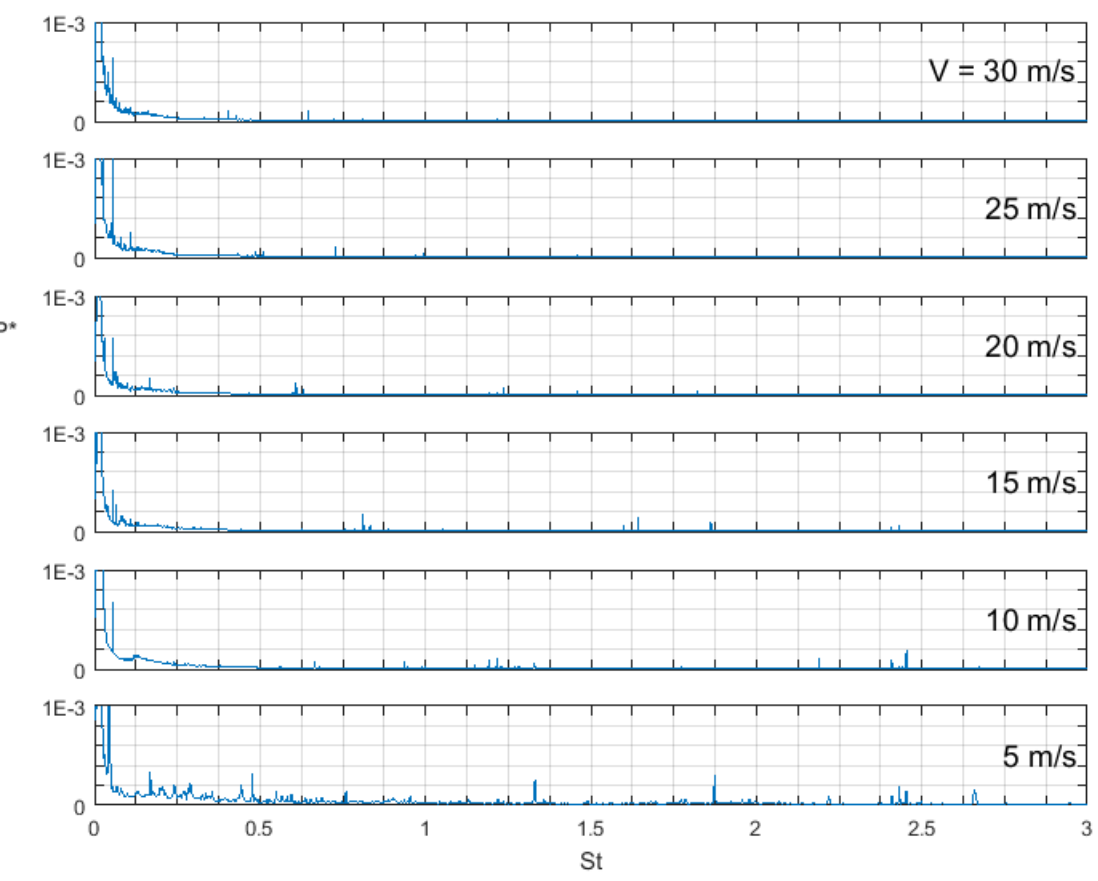

Figure 81: Frequency spectra for a gap width of $6.35 \mathrm{~mm}$, an angle of incidence of 0 degrees, and a flow velocity of 5 to $30 \mathrm{~m} / \mathrm{s}$. 


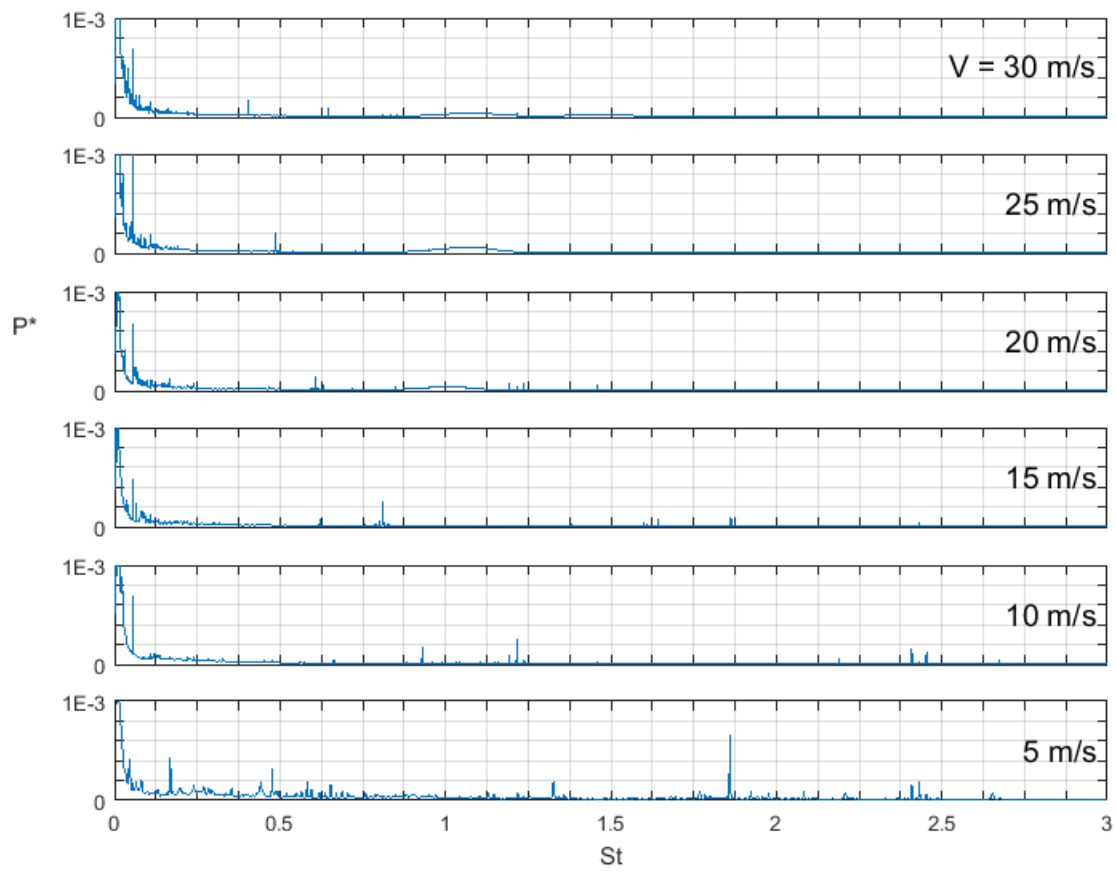

Figure 82: Frequency spectra for a gap width of $6.35 \mathrm{~mm}$, an angle of incidence of 5 degrees, and a flow velocity of 5 to $30 \mathrm{~m} / \mathrm{s}$.

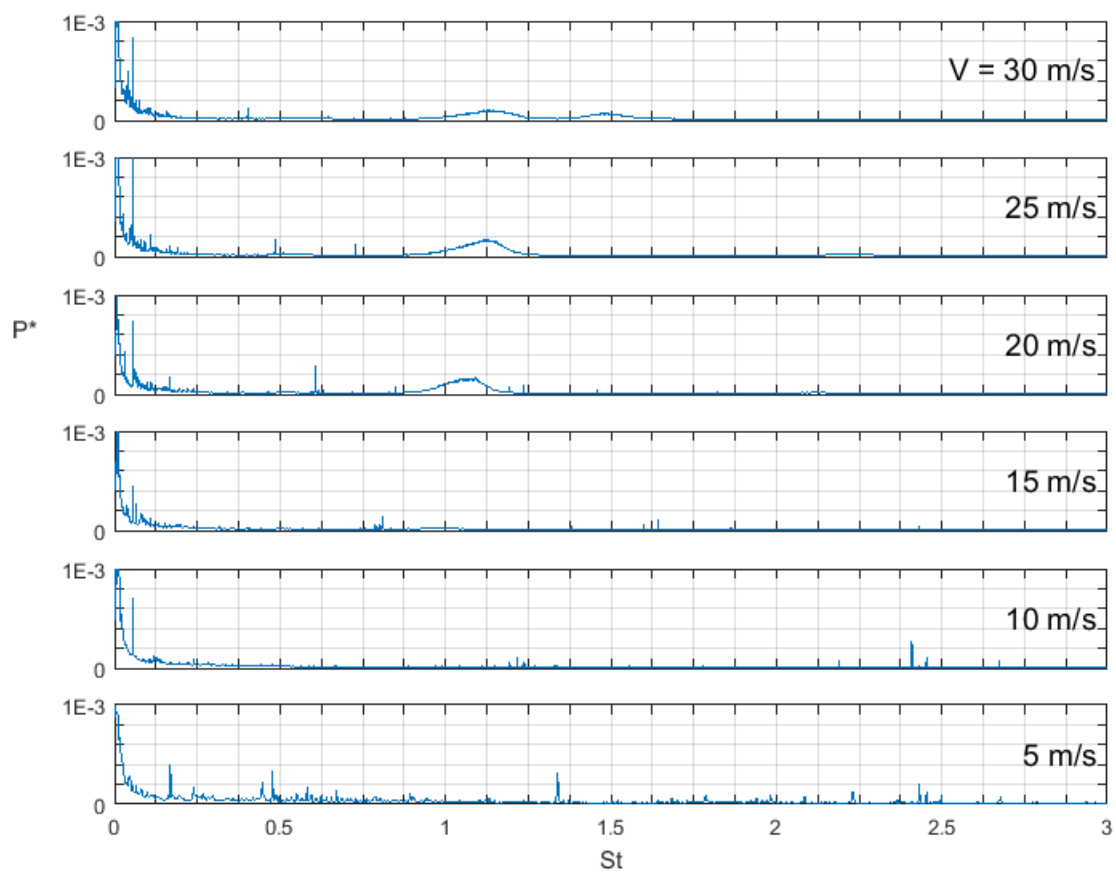

Figure 83: Frequency spectra for a gap width of $6.35 \mathrm{~mm}$, an angle of incidence of 10 degrees, and a flow velocity of 5 to $30 \mathrm{~m} / \mathrm{s}$. 


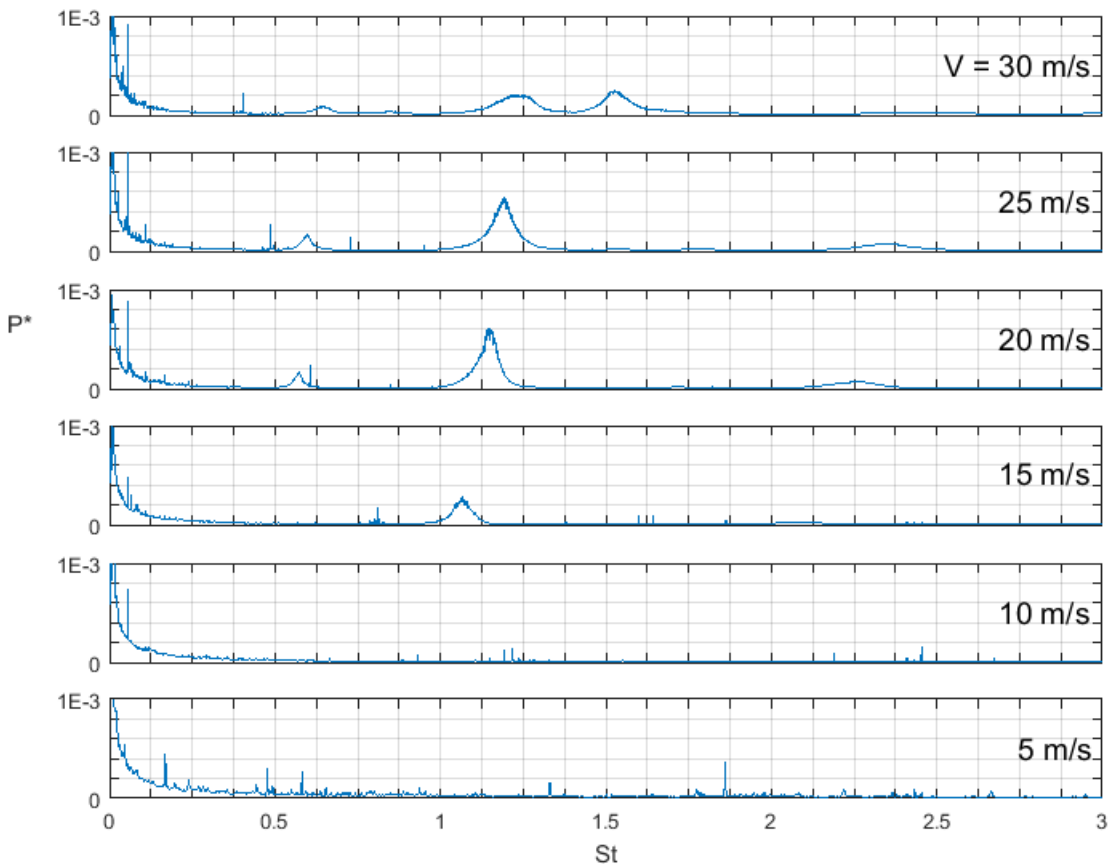

Figure 84: Frequency spectra for a gap width of $6.35 \mathrm{~mm}$, an angle of incidence of 15 degrees, and a flow velocity of 5 to $30 \mathrm{~m} / \mathrm{s}$.
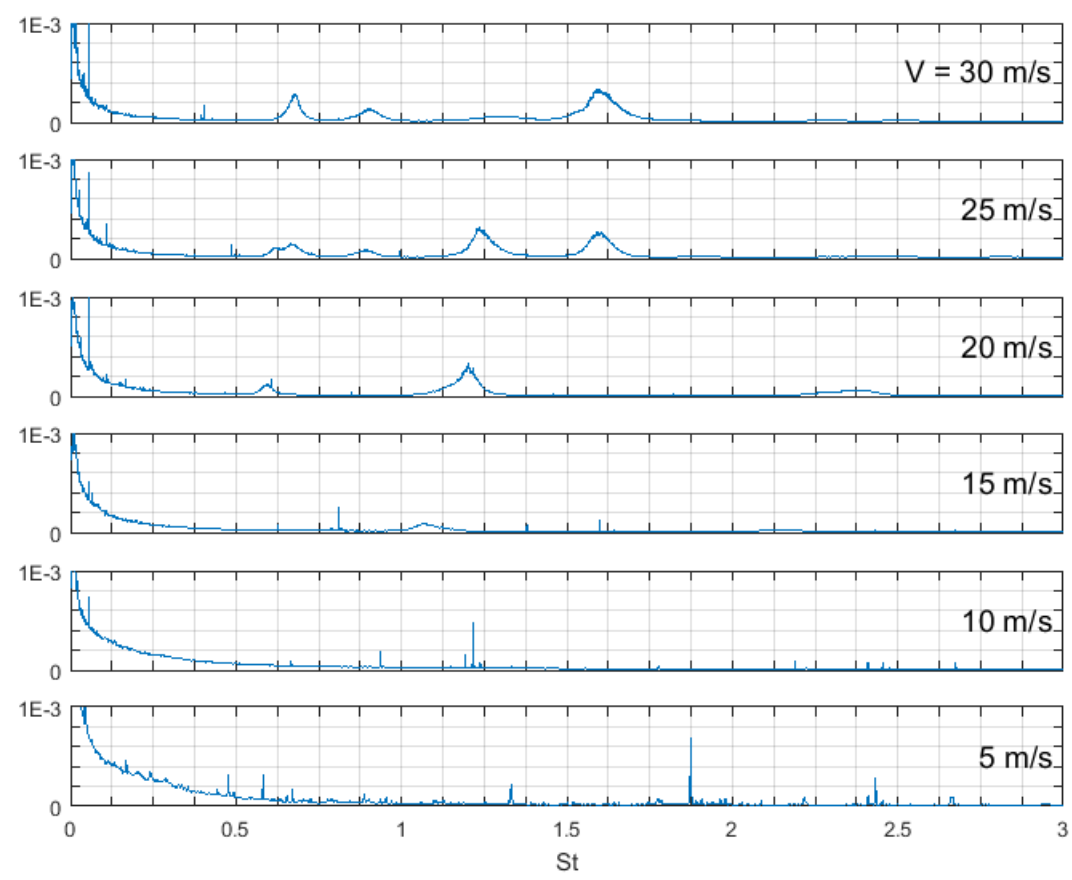

Figure 85: Frequency spectra for a gap width of $6.35 \mathrm{~mm}$, an angle of incidence of 20 degrees, and a flow velocity of 5 to $30 \mathrm{~m} / \mathrm{s}$. 


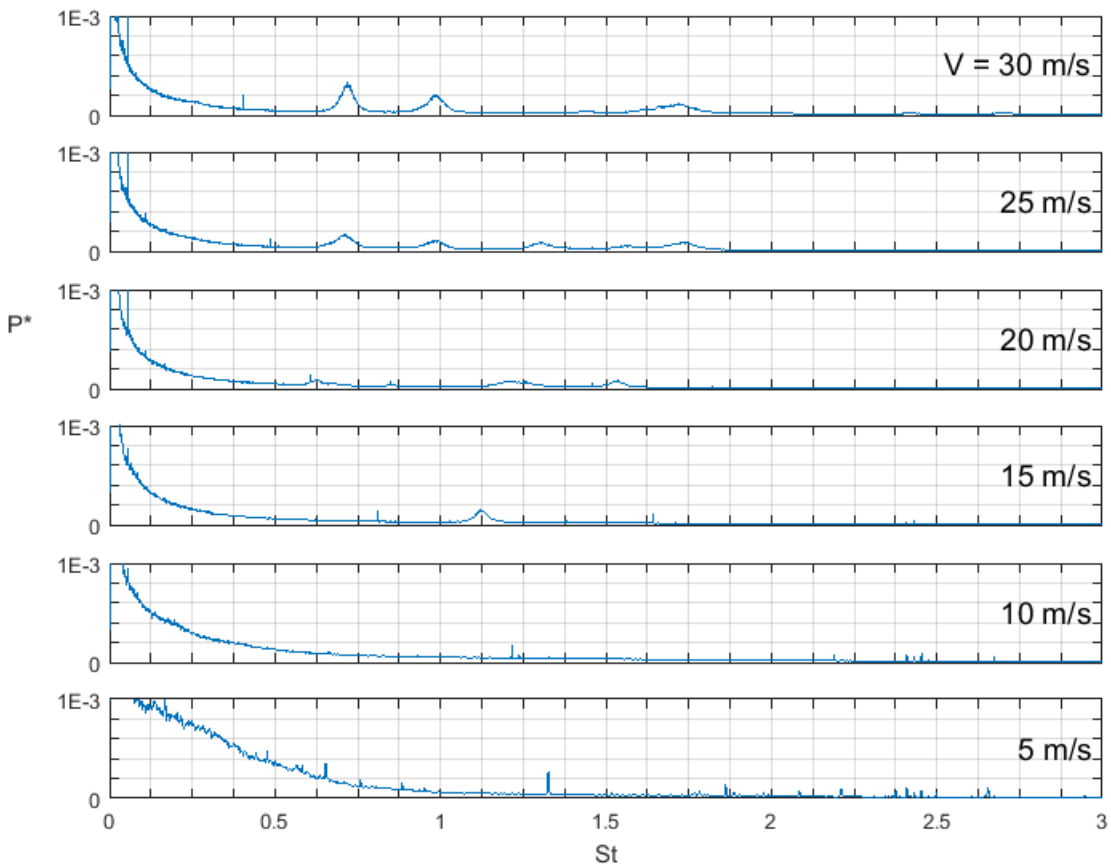

Figure 86: Frequency spectra for a gap width of $6.35 \mathrm{~mm}$, an angle of incidence of 25 degrees, and a flow velocity of 5 to $30 \mathrm{~m} / \mathrm{s}$.

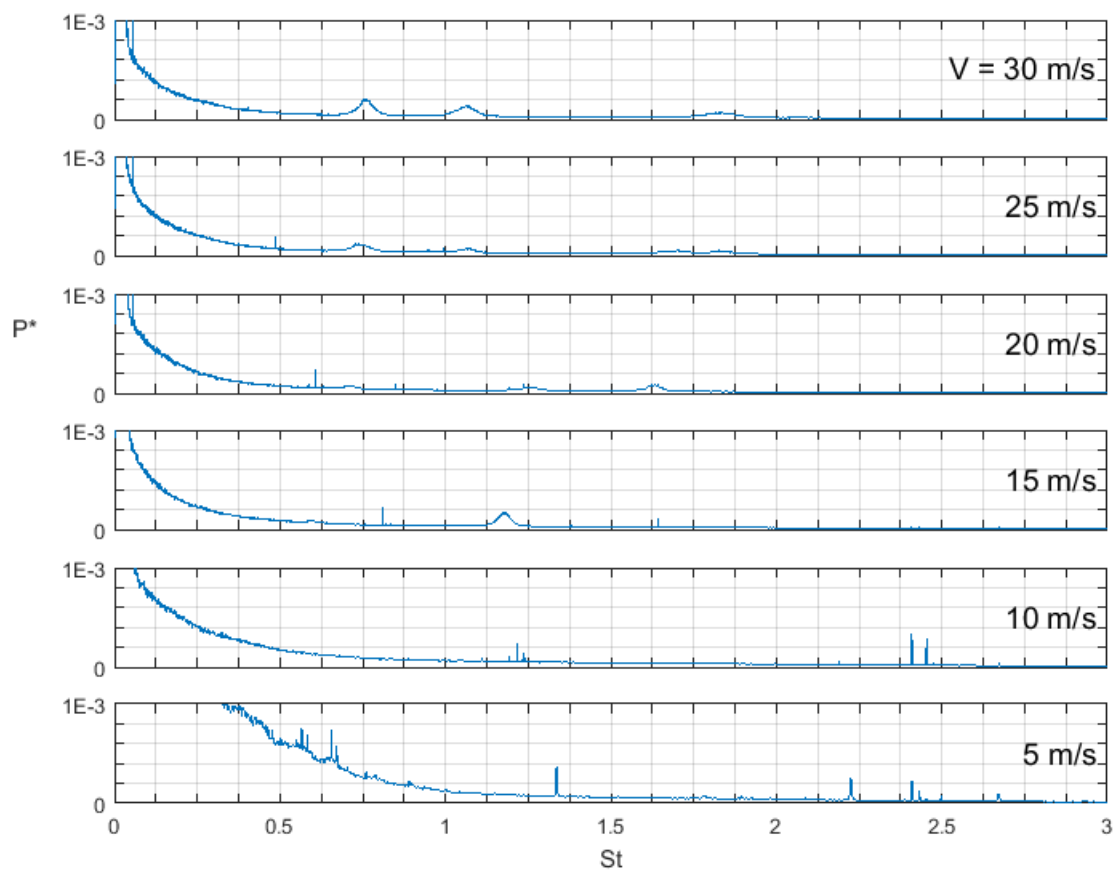

Figure 87: Frequency spectra for a gap width of $6.35 \mathrm{~mm}$, an angle of incidence of 30 degrees, and a flow velocity of 5 to $30 \mathrm{~m} / \mathrm{s}$. 


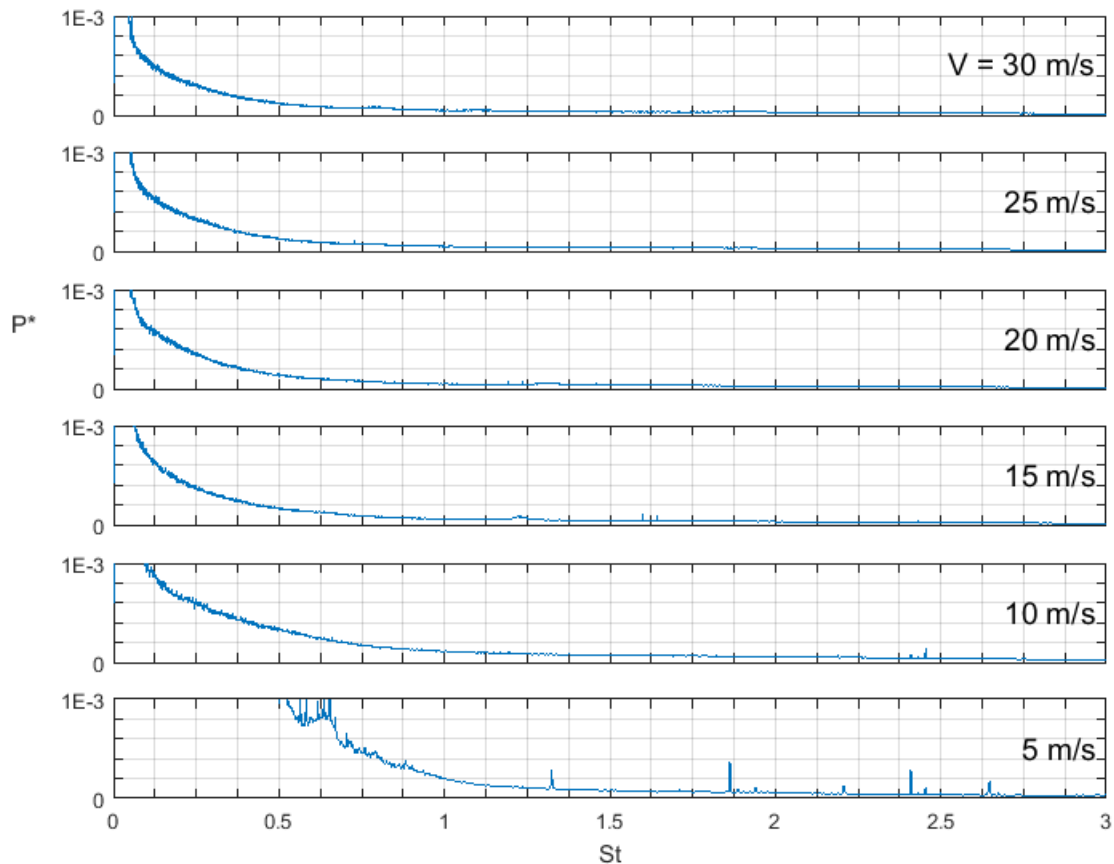

Figure 88: Frequency spectra for a gap width of $6.35 \mathrm{~mm}$, an angle of incidence of 35 degrees, and a flow velocity of 5 to $30 \mathrm{~m} / \mathrm{s}$.
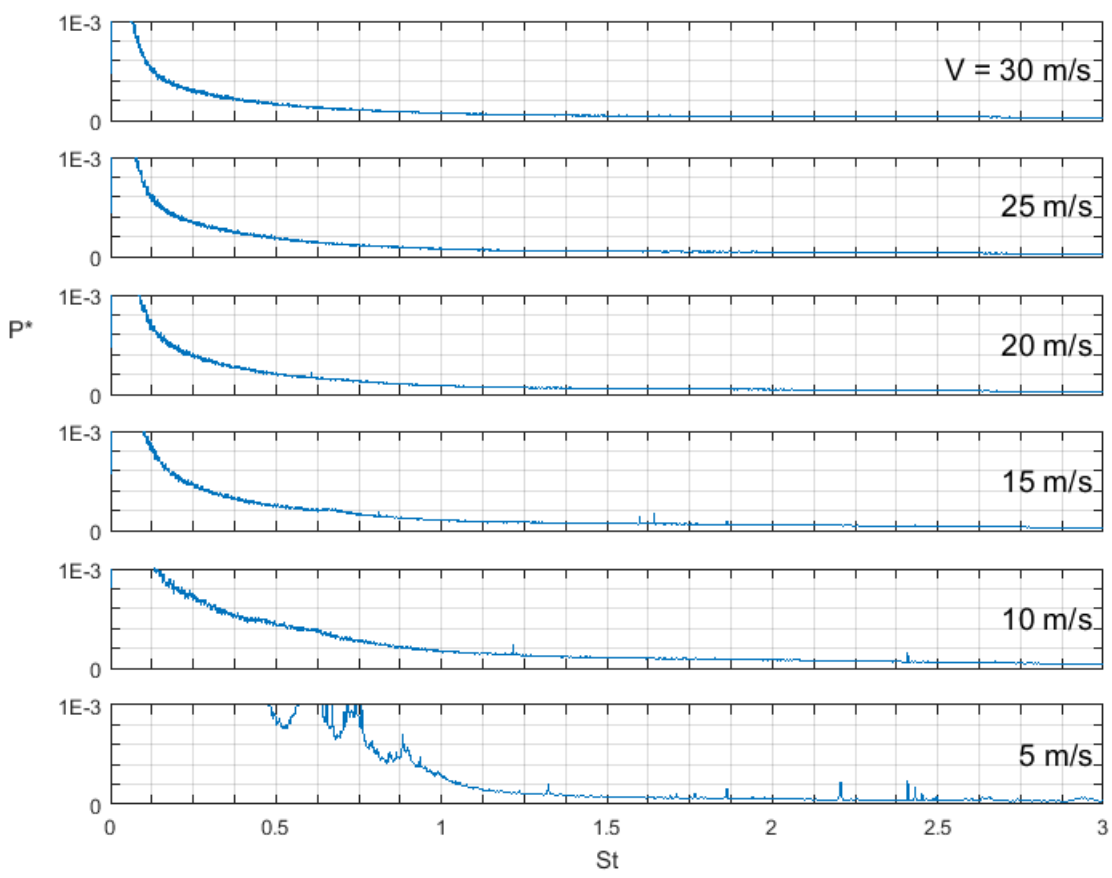

Figure 89: Frequency spectra for a gap width of $6.35 \mathrm{~mm}$, an angle of incidence of 40 degrees, and a flow velocity of 5 to $30 \mathrm{~m} / \mathrm{s}$. 


\section{B.2.3. Effect of flow velocity, gap width $=12.7 \mathrm{~mm}$}

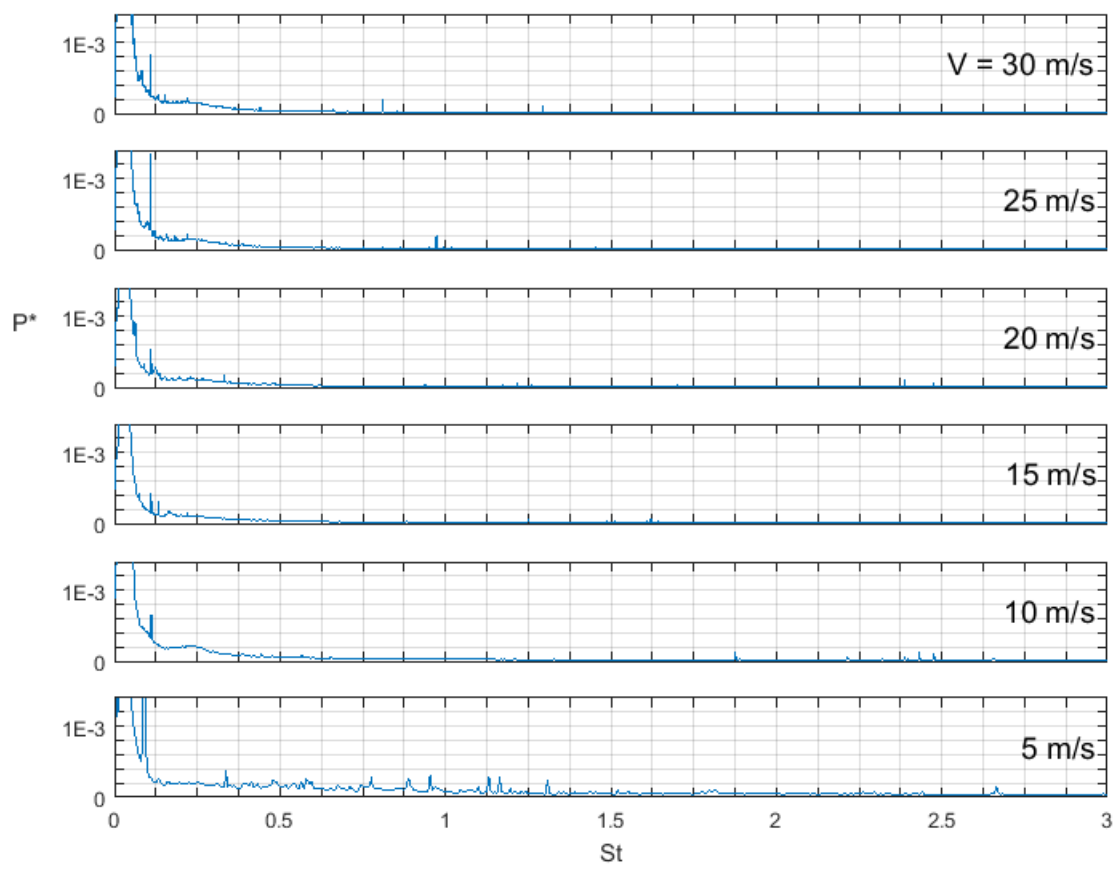

Figure 90: Frequency spectra for a gap width of $12.7 \mathrm{~mm}$, an angle of incidence of 0 degrees, and a flow velocity of 5 to $30 \mathrm{~m} / \mathrm{s}$. 


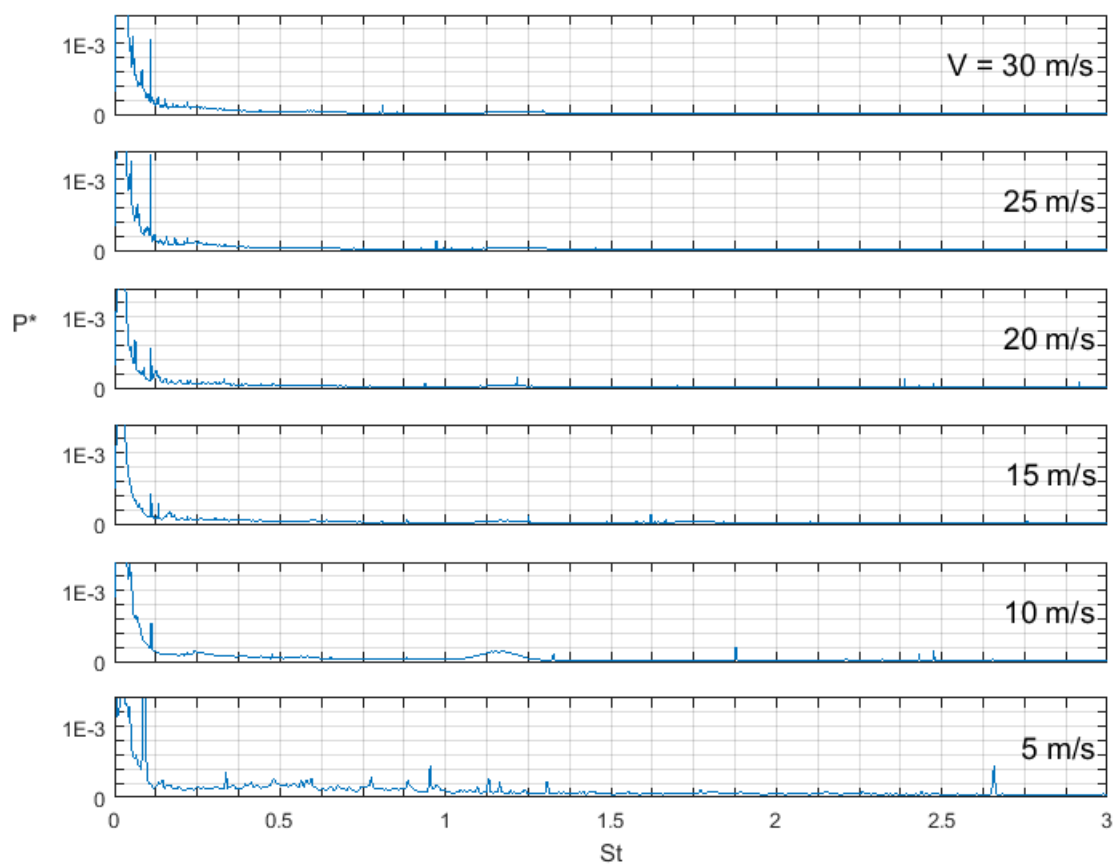

Figure 91: Frequency spectra for a gap width of $12.7 \mathrm{~mm}$, an angle of incidence of 5 degrees, and a flow velocity of 5 to $30 \mathrm{~m} / \mathrm{s}$.
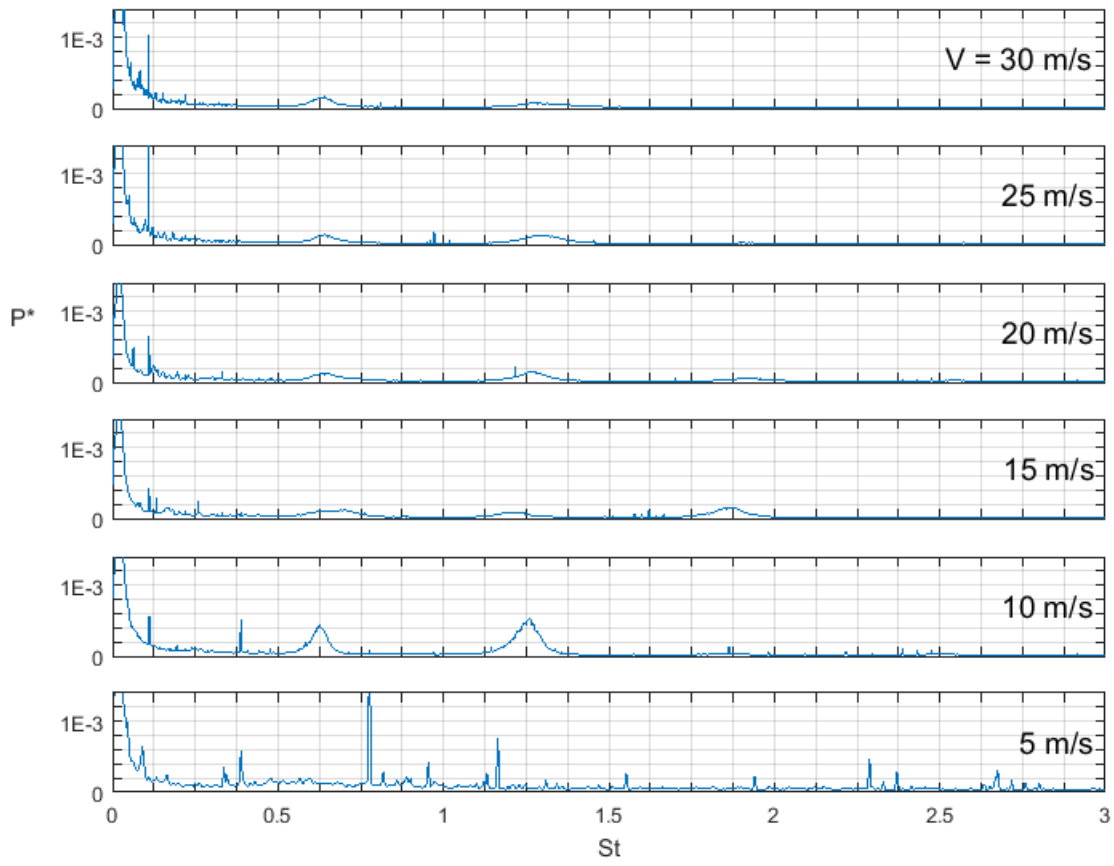

Figure 92: Frequency spectra for a gap width of $12.7 \mathrm{~mm}$, an angle of incidence of 10 degrees, and a flow velocity of 5 to $30 \mathrm{~m} / \mathrm{s}$. 


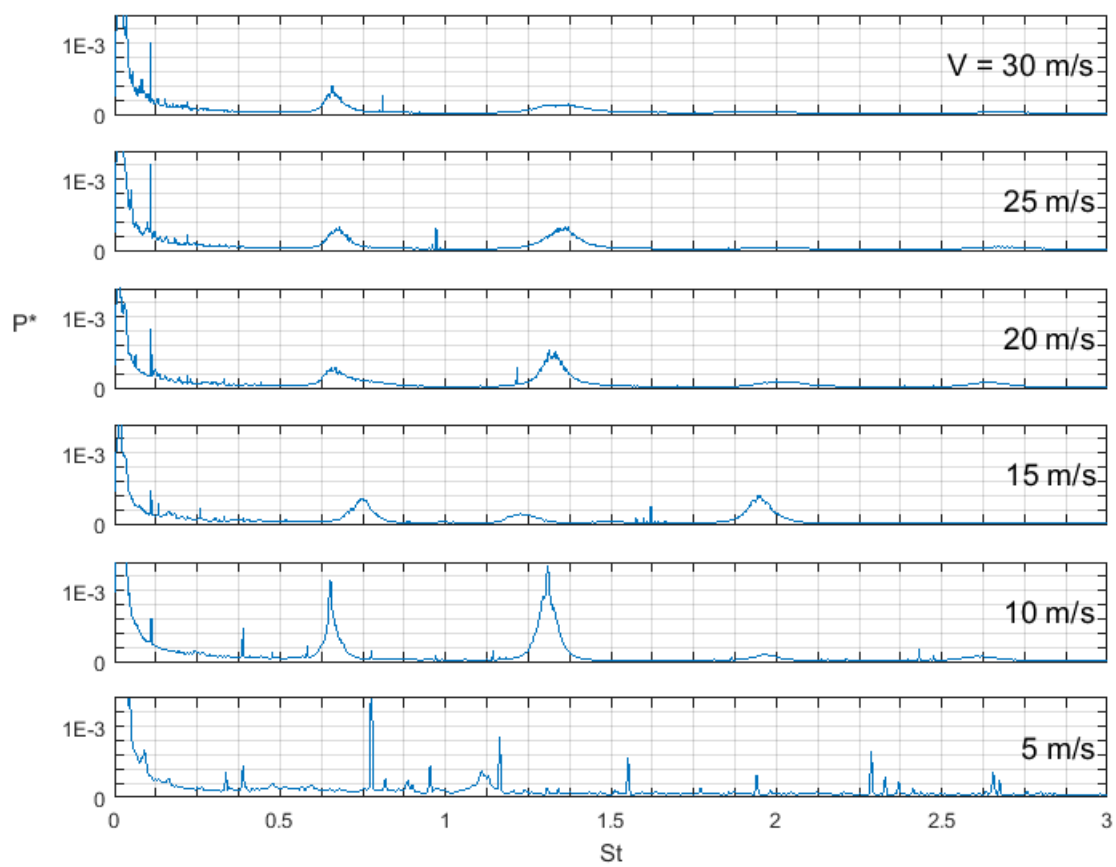

Figure 93: Frequency spectra for a gap width of $12.7 \mathrm{~mm}$, an angle of incidence of 15 degrees, and a flow velocity of 5 to $30 \mathrm{~m} / \mathrm{s}$.
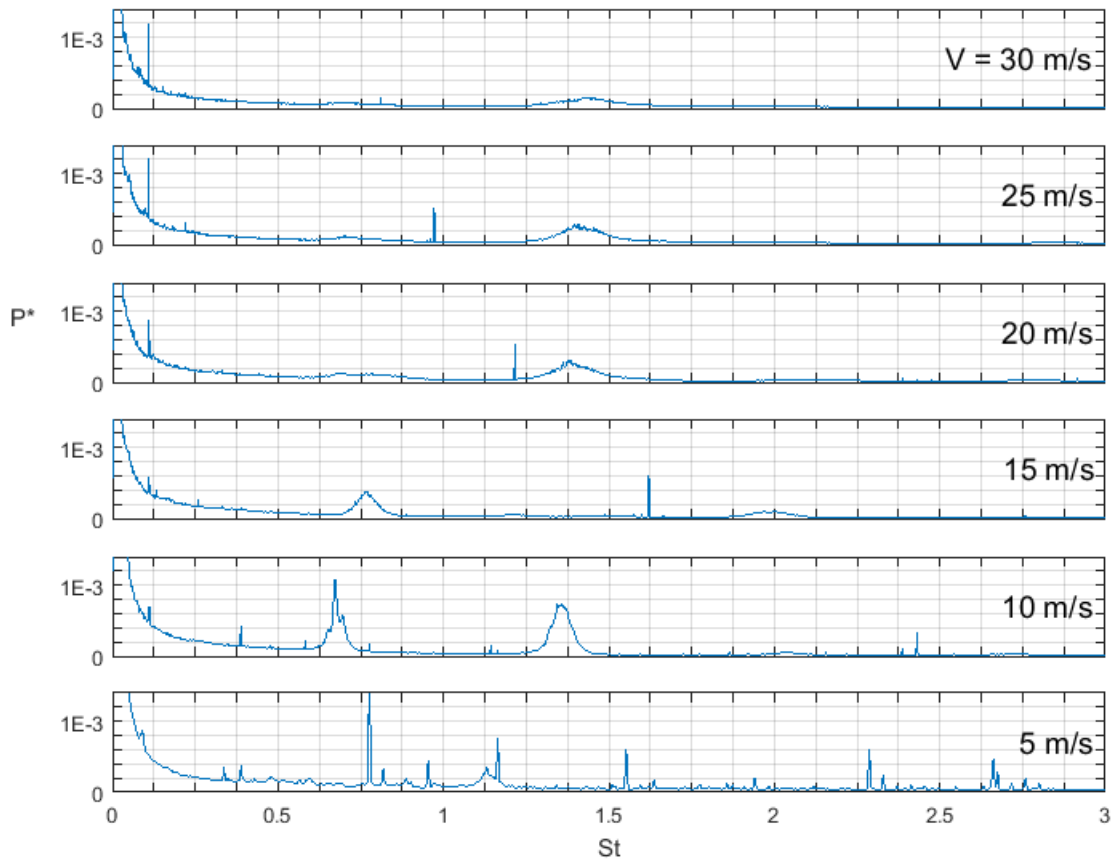

Figure 94: Frequency spectra for a gap width of $12.7 \mathrm{~mm}$, an angle of incidence of 20 degrees, and a flow velocity of 5 to $30 \mathrm{~m} / \mathrm{s}$. 


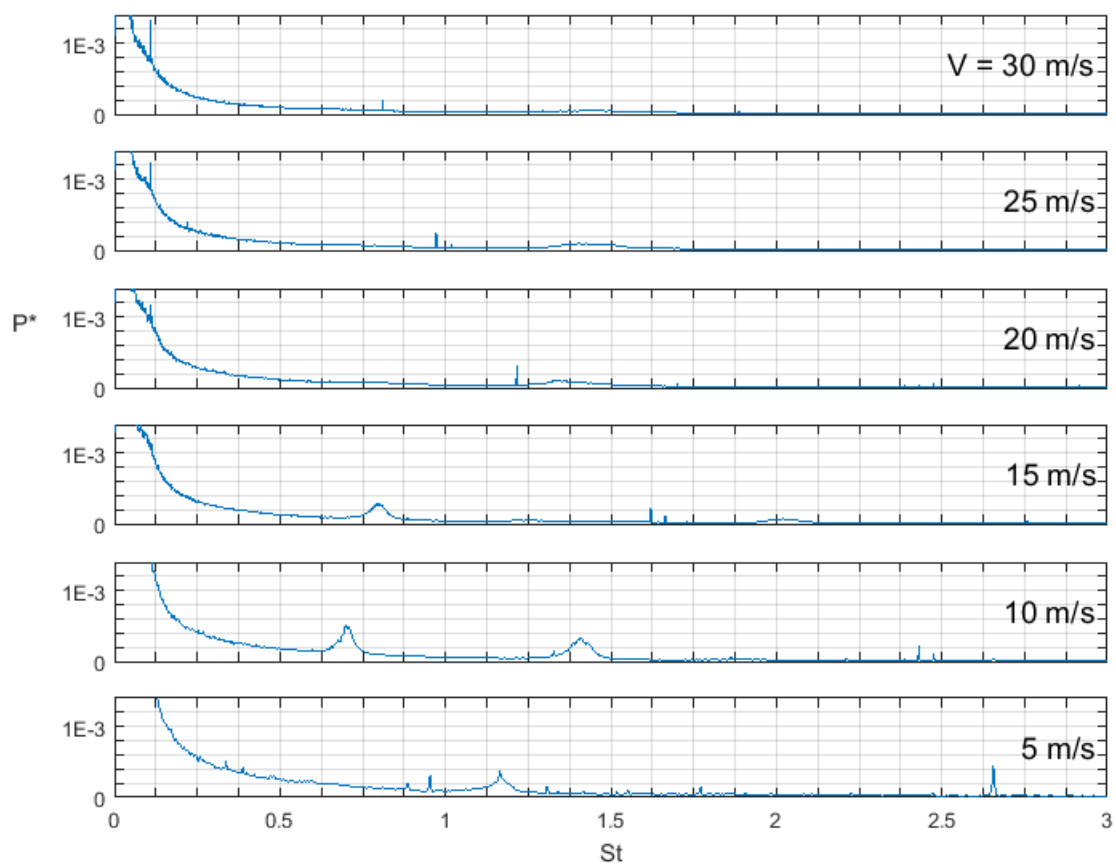

Figure 95: Frequency spectra for a gap width of $12.7 \mathrm{~mm}$, an angle of incidence of 25 degrees, and a flow velocity of 5 to $30 \mathrm{~m} / \mathrm{s}$.
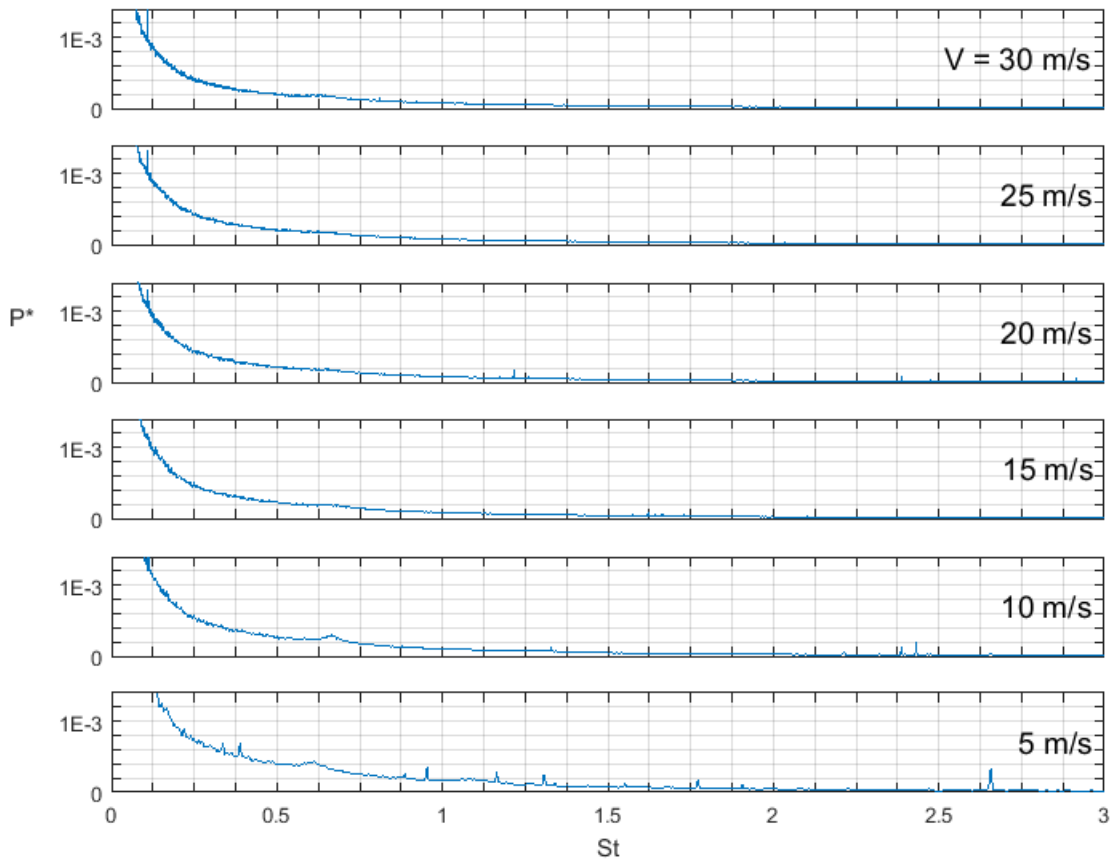

Figure 96: Frequency spectra for a gap width of $12.7 \mathrm{~mm}$, an angle of incidence of 30 degrees, and a flow velocity of 5 to $30 \mathrm{~m} / \mathrm{s}$. 


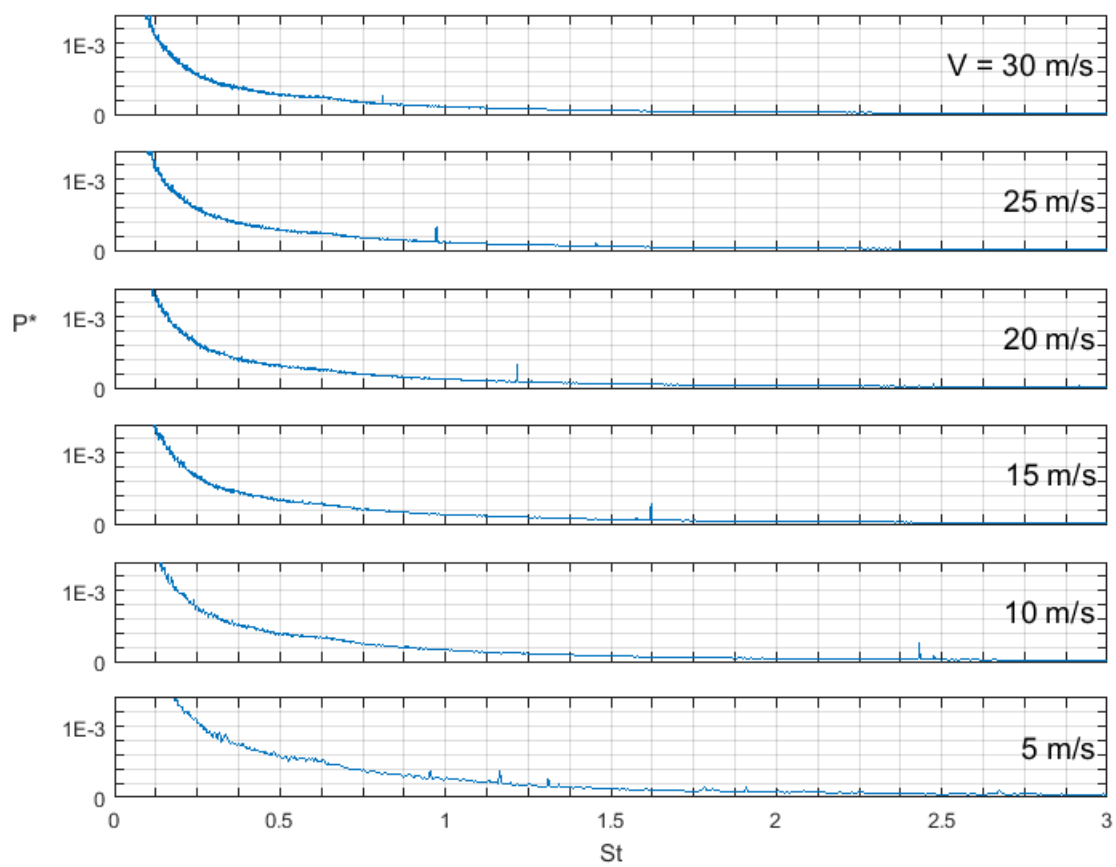

Figure 97: Frequency spectra for a gap width of $12.7 \mathrm{~mm}$, an angle of incidence of 35 degrees, and a flow velocity of 5 to $30 \mathrm{~m} / \mathrm{s}$.
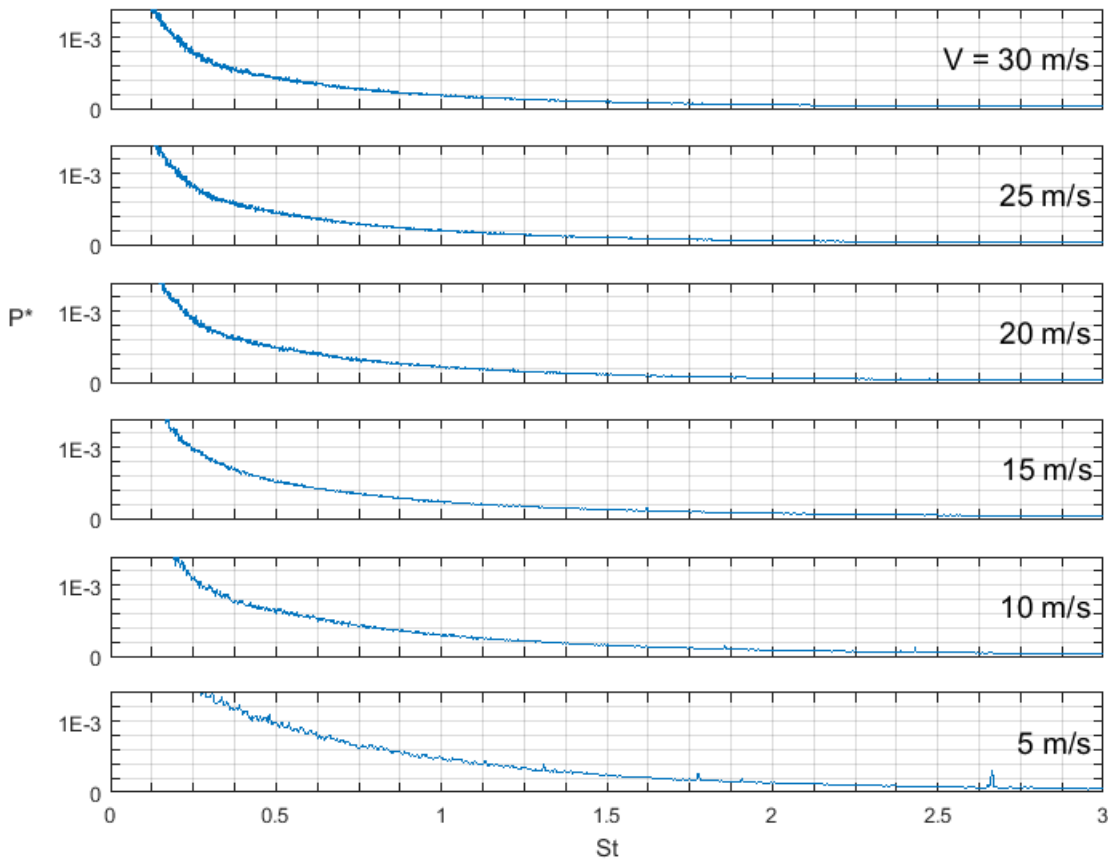

Figure 98: Frequency spectra for a gap width of $12.7 \mathrm{~mm}$, an angle of incidence of 40 degrees, and a flow velocity of 5 to $30 \mathrm{~m} / \mathrm{s}$. 


\section{B.2.4. Effect of streamwise position}

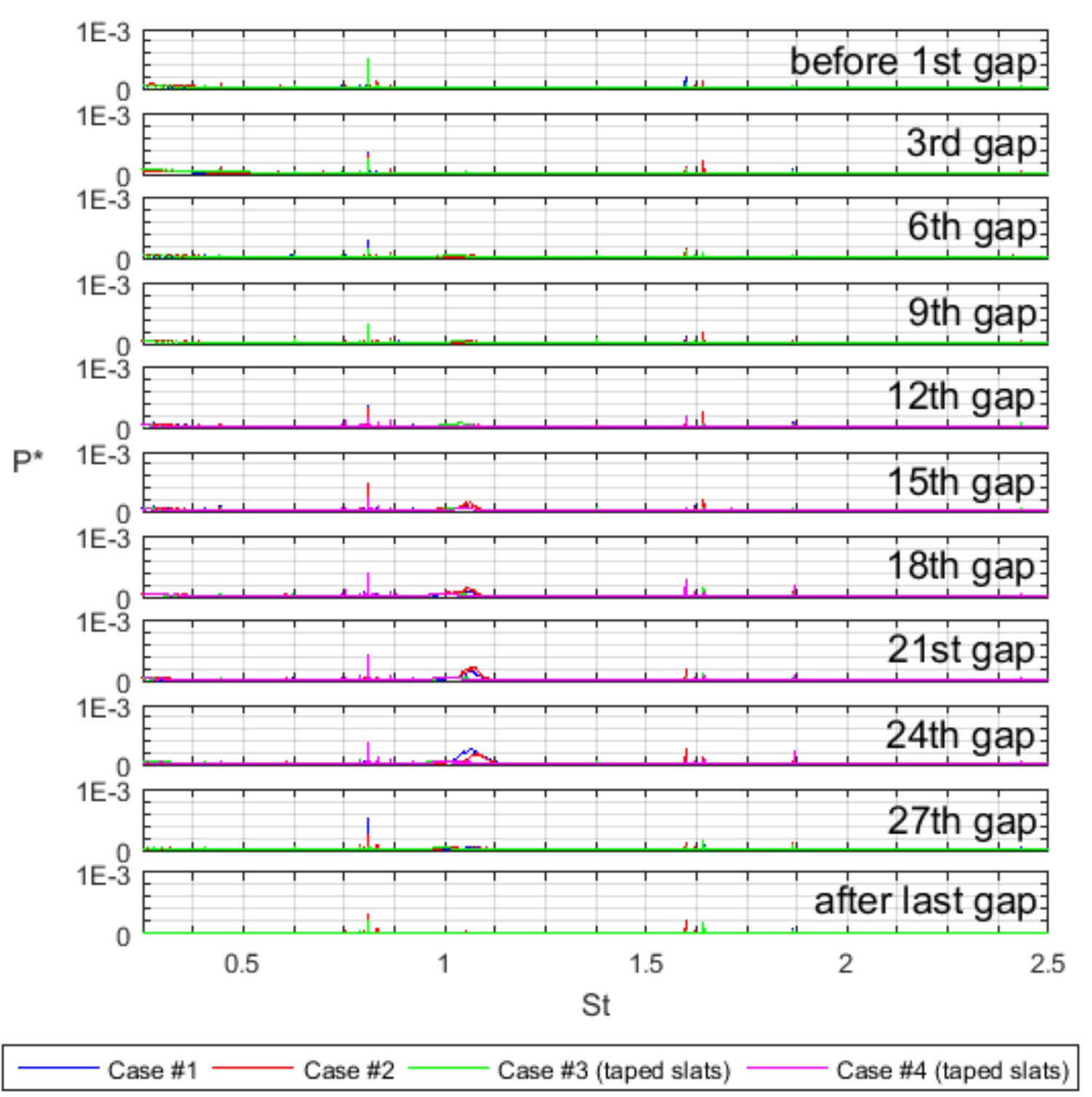

Figure 99: Frequency spectra for a gap width of $6.35 \mathrm{~mm}$, an angle of incidence of $15^{\circ}$, and a flow velocity of $15 \mathrm{~m} / \mathrm{s}$. Results are shown for un-taped (blue \& red) and taped (green \& pink) slats. The streamwise position is varied, from before the first gap to after the last gap. For Case \#4, spectra are only shown for the $12^{\text {th }}$ to $24^{\text {th }}$ gaps. 


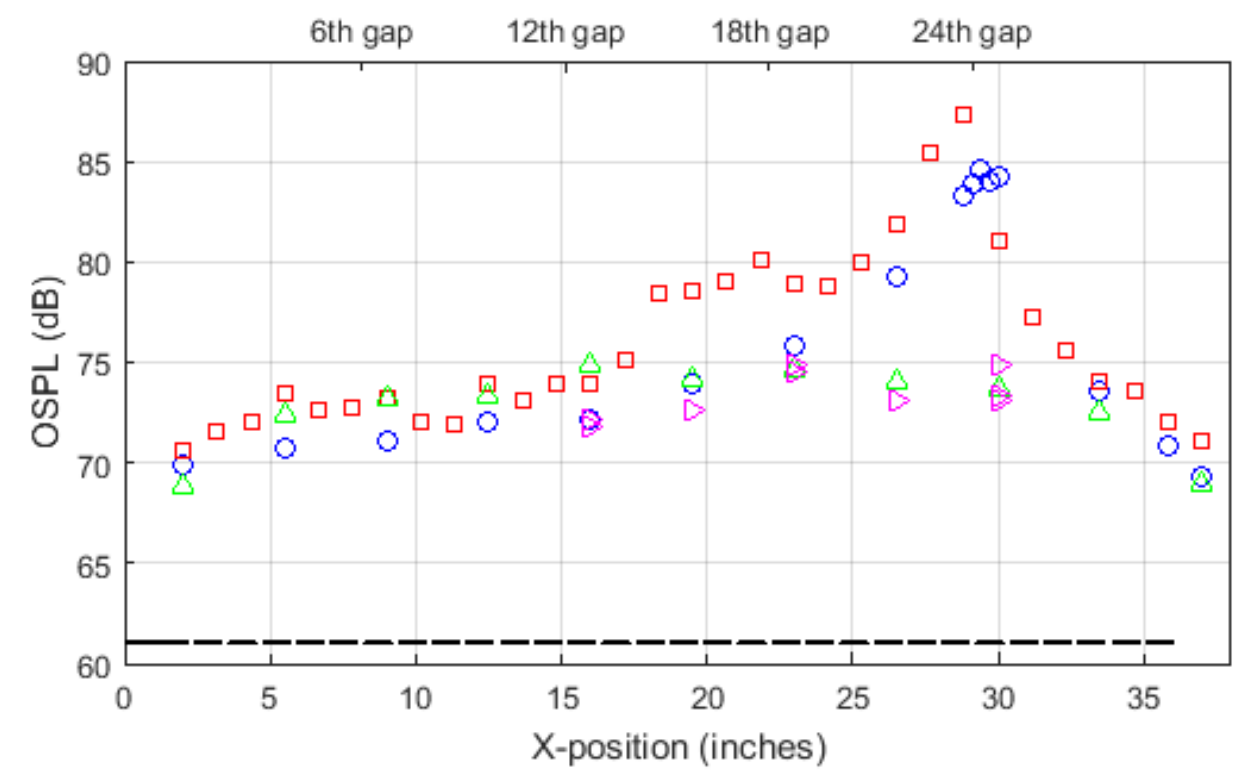

\begin{tabular}{lllllll}
\hline & JUN 2016 & AUG 2016 & $\triangle$ & OCT 2016 (taped) & $\square$ & JAN/FEB 2017 (taped)
\end{tabular}

Figure 100: Overall sound pressure level vs. streamwise position for a gap width of $6.35 \mathrm{~mm}$, an angle of incidence of $15^{\circ}$, and a flow velocity of $15 \mathrm{~m} / \mathrm{s}$. Results are shown for un-taped (blue $\&$ red) and taped (green $\&$ pink) slats. 


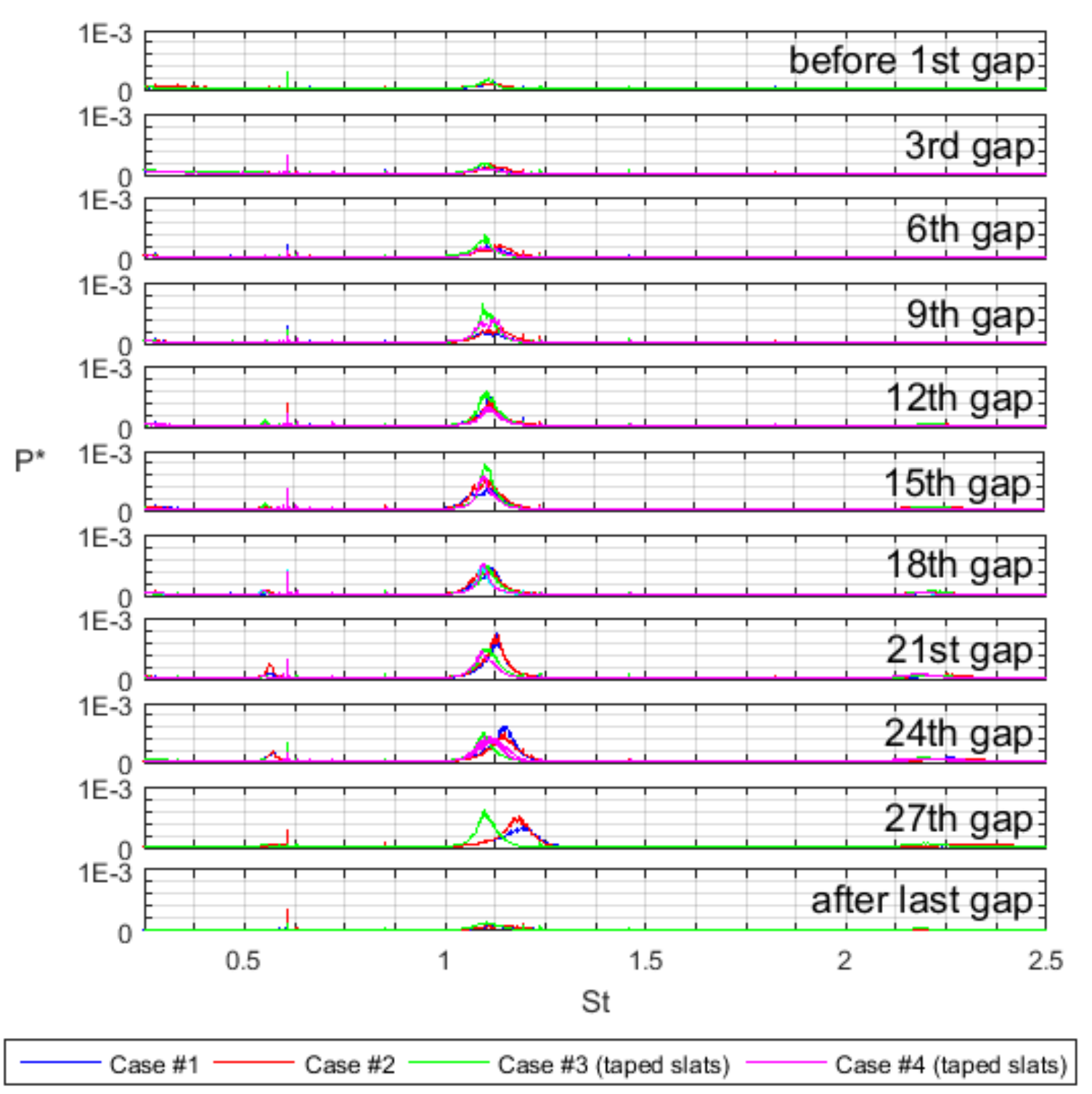

Figure 101: Frequency spectra for a gap width of $6.35 \mathrm{~mm}$, an angle of incidence of $15^{\circ}$, and a flow velocity of $20 \mathrm{~m} / \mathrm{s}$. Results are shown for un-taped (blue $\&$ red) and taped (green \& pink) slats. The streamwise position is varied, from before the first gap to after the last gap. For Case \#4, spectra are only shown for the $3^{\text {rd }}$ to $24^{\text {th }}$ gaps. 


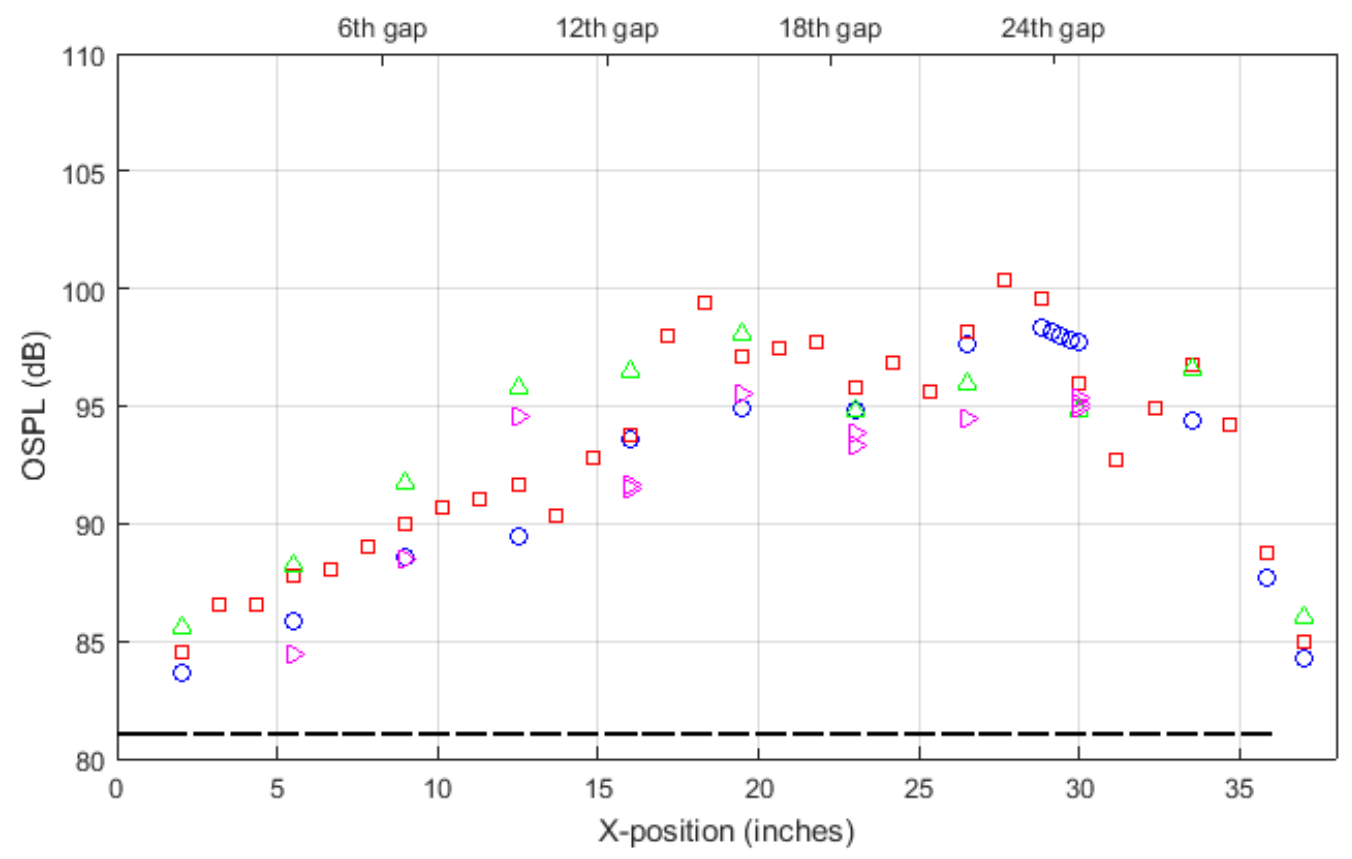

\begin{tabular}{lllllll}
\hline & Case \#1 & $\square$ & Case \#2 & $\triangle$ & Case \#3 (taped slats) $\quad \square \quad$ Case \#4 (taped slats)
\end{tabular}

Figure 102: Overall sound pressure level vs. streamwise position for a gap width of $6.35 \mathrm{~mm}$, an angle of incidence of $15^{\circ}$, and a flow velocity of $20 \mathrm{~m} / \mathrm{s}$. Results are shown for un-taped (blue \& red) and taped (green \& pink) slats. 


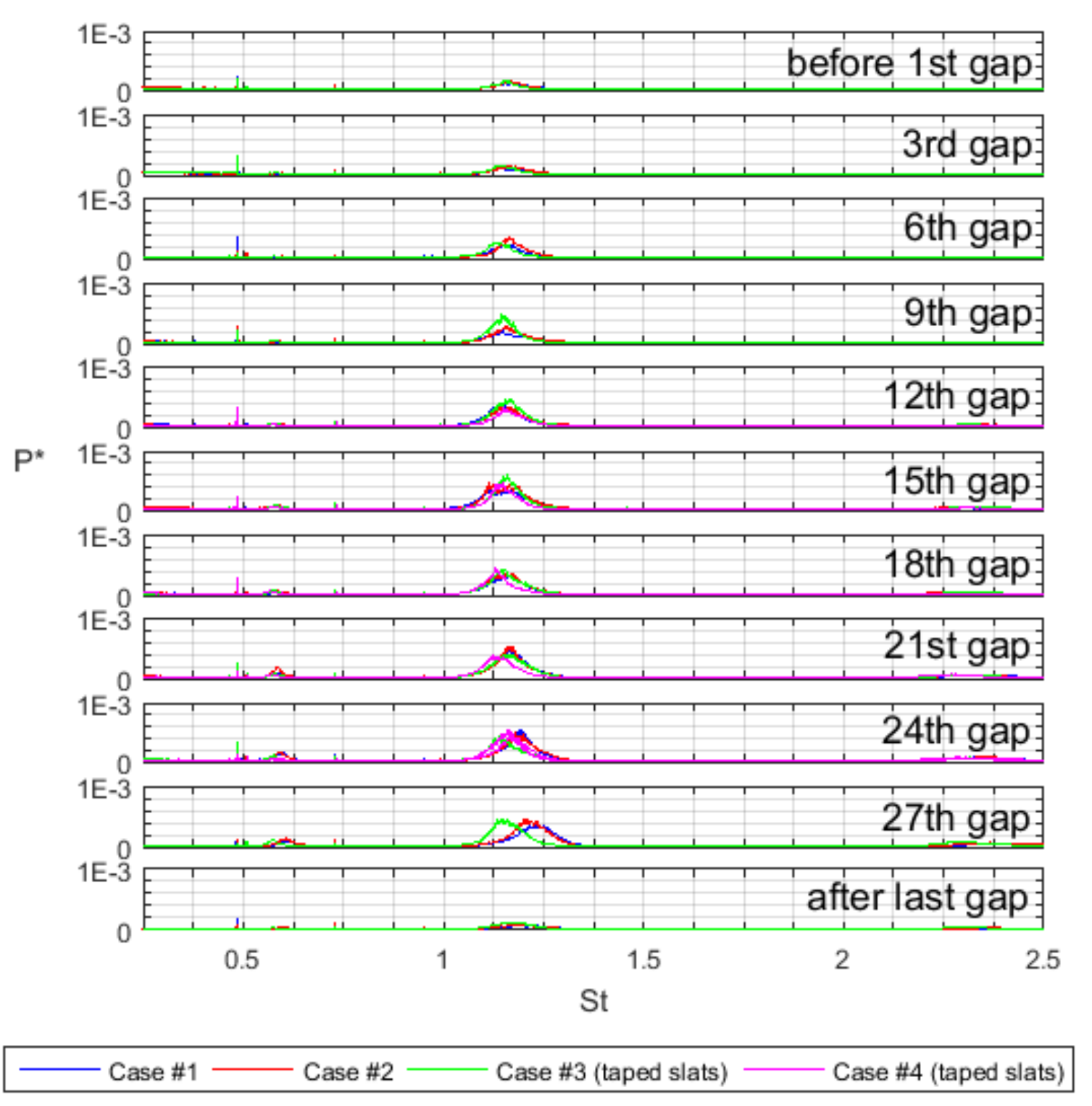

Figure 103: Frequency spectra for a gap width of $6.35 \mathrm{~mm}$, an angle of incidence of $15^{\circ}$, and a flow velocity of $25 \mathrm{~m} / \mathrm{s}$. Results are shown for un-taped (blue $\&$ red) and taped (green $\&$ pink) slats. The streamwise position is varied, from before the first gap to after the last gap. For Case \#4, spectra are only shown for the $3^{\text {rd }}$ to $24^{\text {th }}$ gaps. 


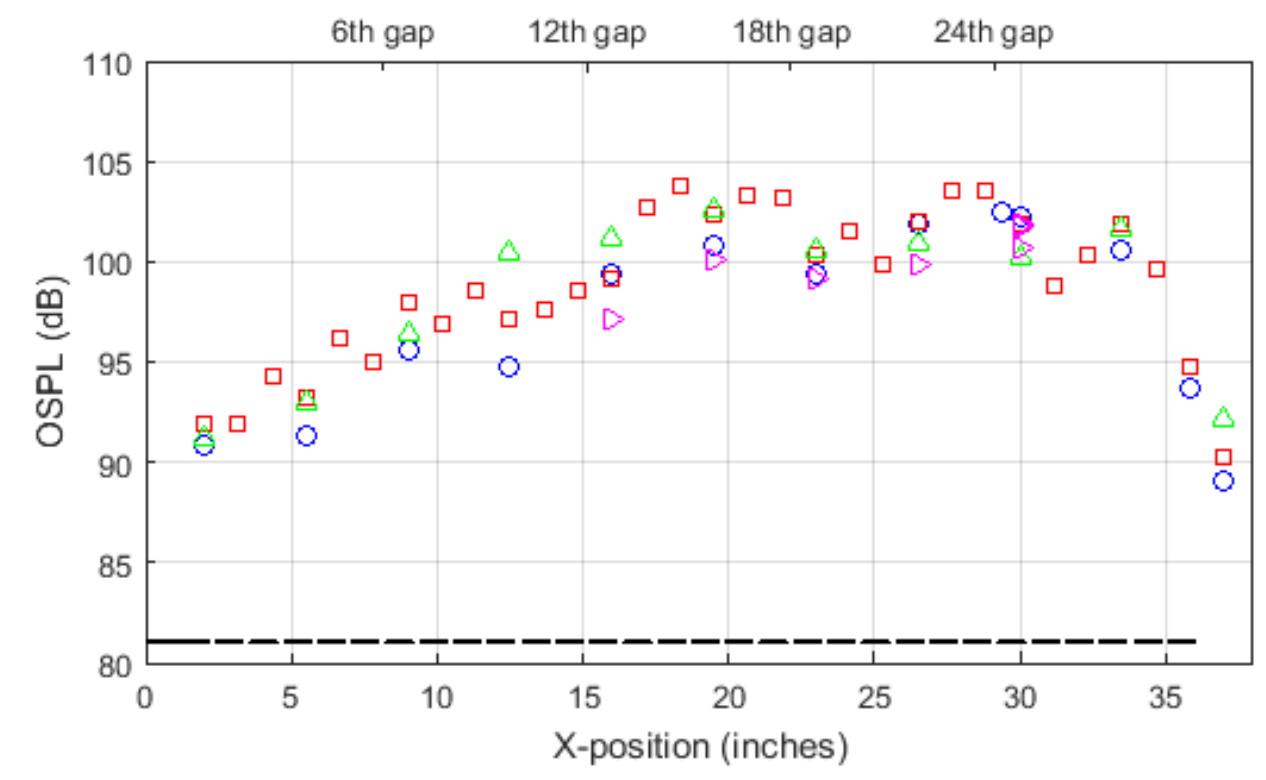

\begin{tabular}{lllllll}
\hline & JUN 2016 & AUG 2016 & $\Delta$ & OCT 2016 (taped) & $\square$ & JAN/FEB 2017 (taped)
\end{tabular}

Figure 104: Overall sound pressure level vs. streamwise position for a gap width of $6.35 \mathrm{~mm}$, an angle of incidence of $15^{\circ}$, and a flow velocity of $25 \mathrm{~m} / \mathrm{s}$. Results are shown for un-taped (blue \& red) and taped (green \& pink) slats. 


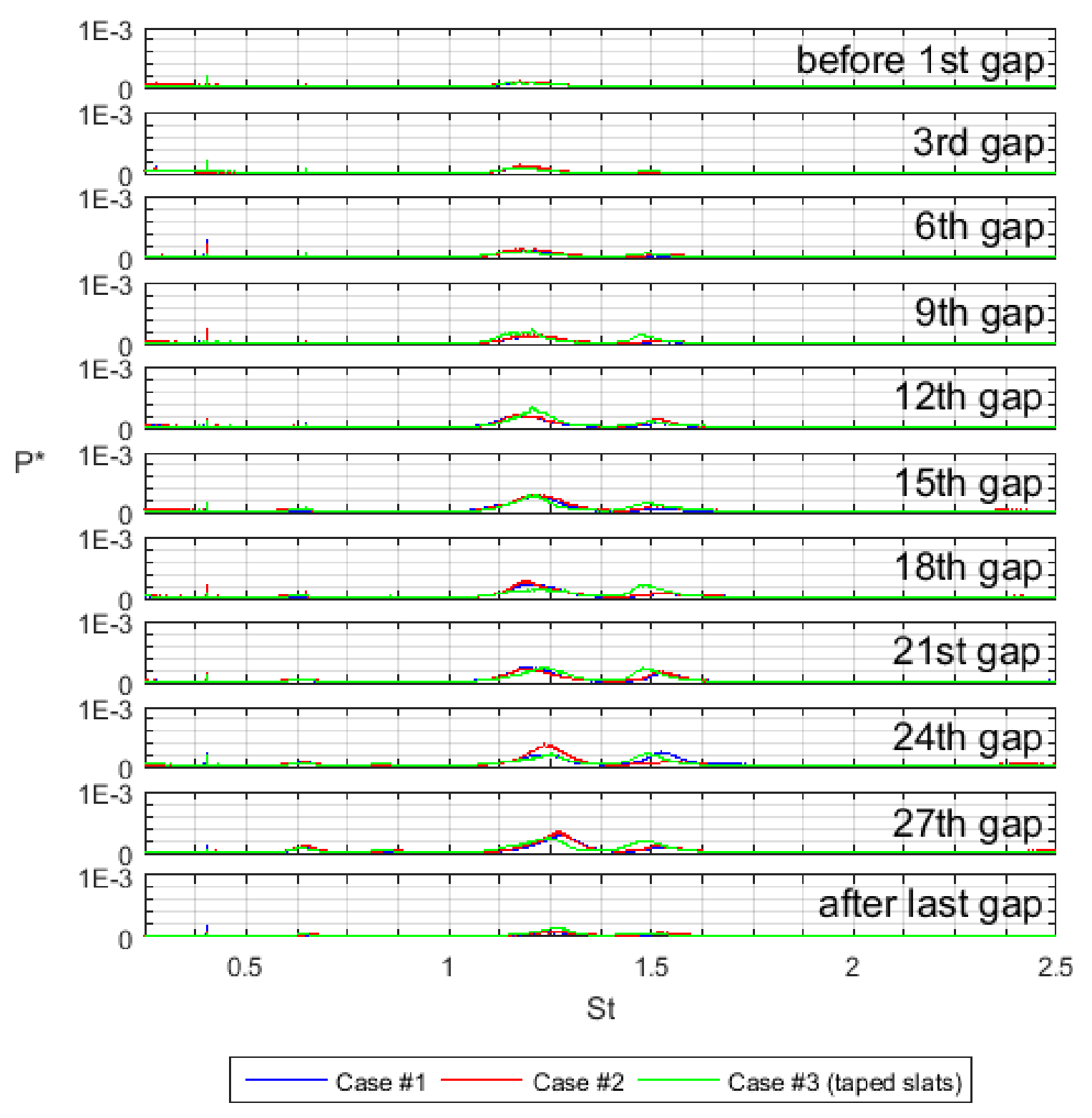

Figure 105: Frequency spectra for a gap width of $6.35 \mathrm{~mm}$, an angle of incidence of $15^{\circ}$, and a flow velocity of $30 \mathrm{~m} / \mathrm{s}$. Results are shown for un-taped (blue \& red) and taped (green \& pink) slats. The streamwise position is varied, from before the first gap to after the last gap. 


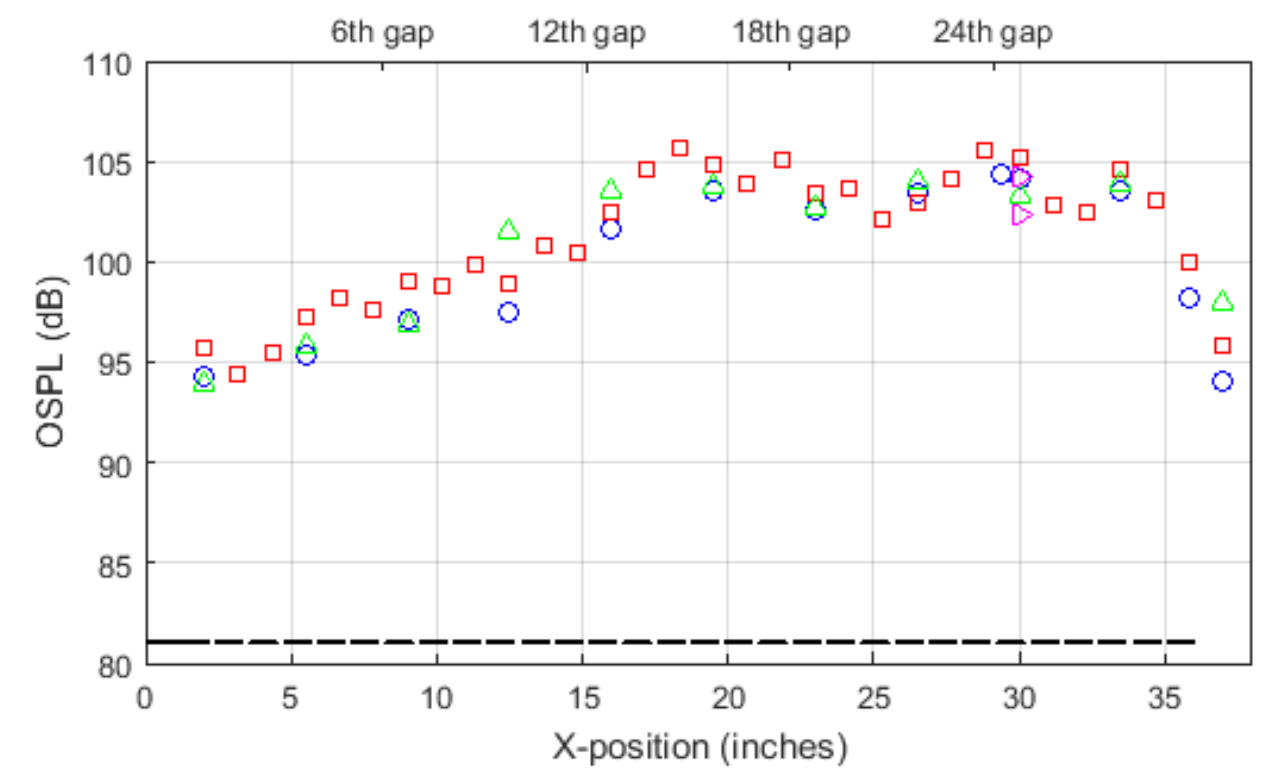

\begin{tabular}{llllllll}
\hline & JUN 2016 & AUG 2016 & $\Delta$ & OCT 2016 (taped) & $\square$ & JAN/FEB 2017 (taped)
\end{tabular}

Figure 106: Overall sound pressure level vs. streamwise position for a gap width of $6.35 \mathrm{~mm}$, an angle of incidence of $15^{\circ}$, and a flow velocity of $30 \mathrm{~m} / \mathrm{s}$. Results are shown for un-taped (blue \& red) and taped (green \& pink) slats. 


\section{B.3. PIV Results}

\section{B.3.1. Effect of angle of incidence}

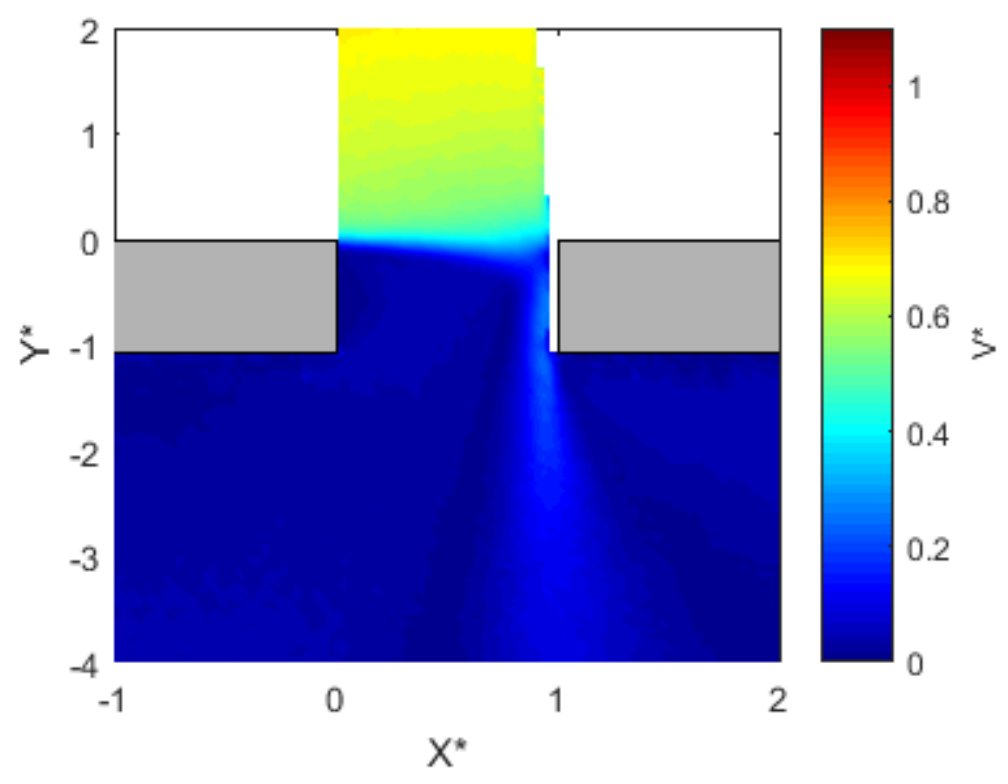

Figure 107: PIV results showing the average velocity field at the 24th gap for a gap width of $6.35 \mathrm{~mm}$, an angle of incidence of 0 degrees, and a flow velocity of $15 \mathrm{~m} / \mathrm{s}$.

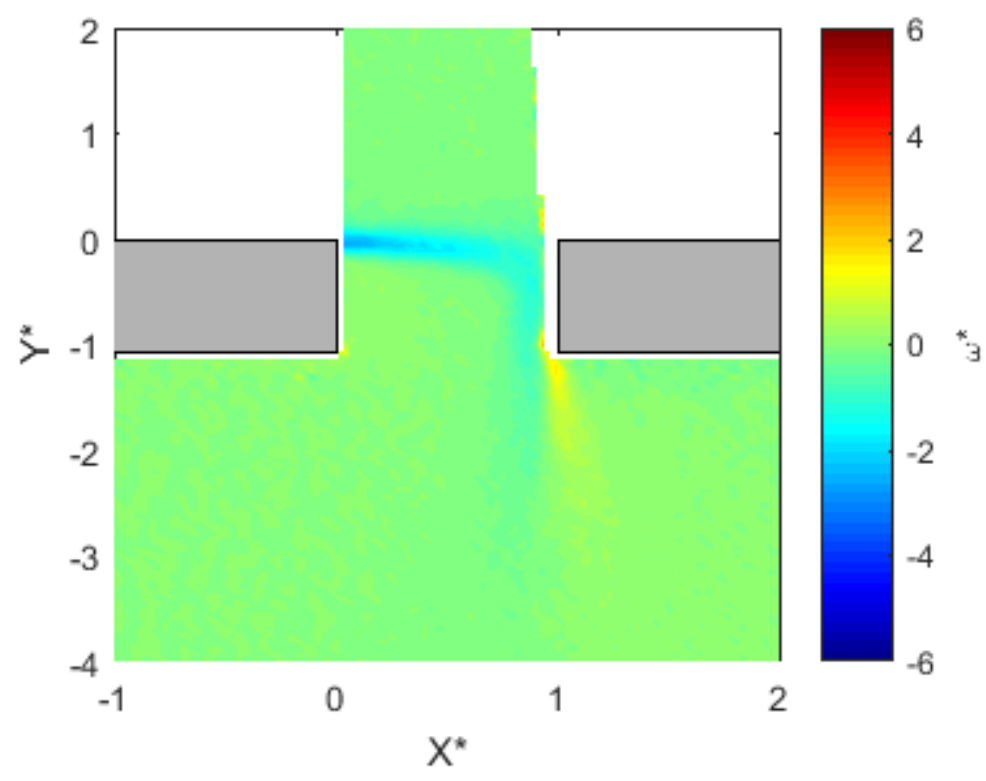

Figure 108: PIV results showing the average vorticity field at the 24th gap for a gap width of $6.35 \mathrm{~mm}$, an angle of incidence of 0 degrees, and a flow velocity of $15 \mathrm{~m} / \mathrm{s}$. 


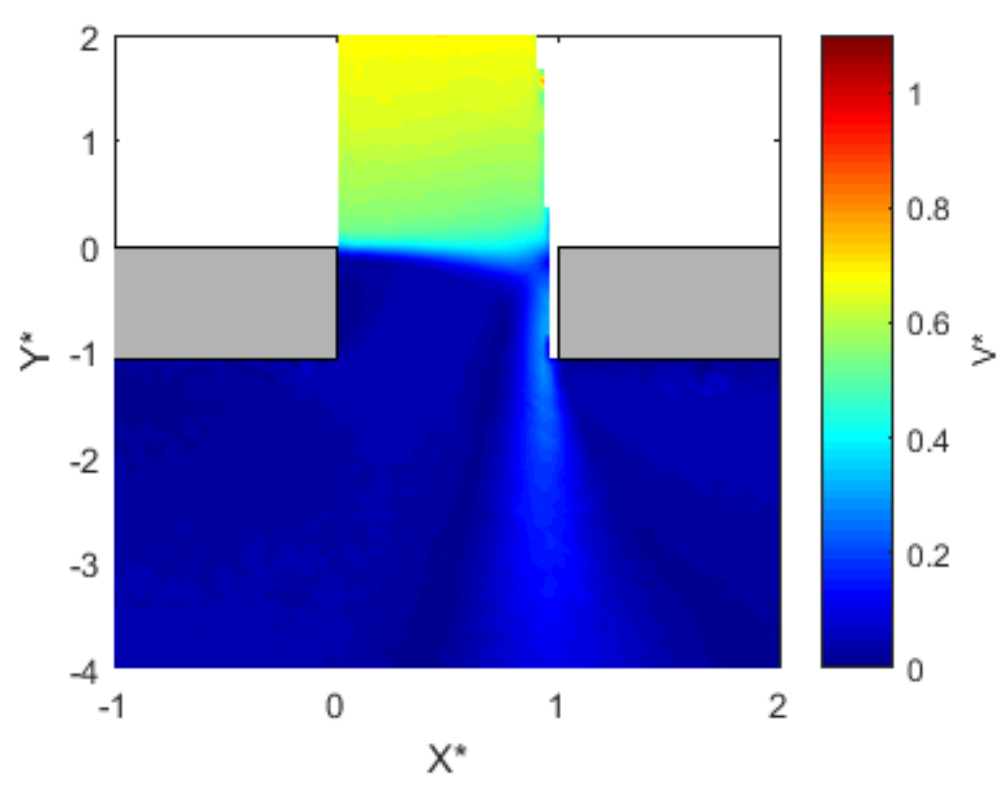

Figure 109: PIV results showing the average velocity field at the 24th gap for a gap width of $6.35 \mathrm{~mm}$, an angle of incidence of 0 degrees, and a flow velocity of $20 \mathrm{~m} / \mathrm{s}$.

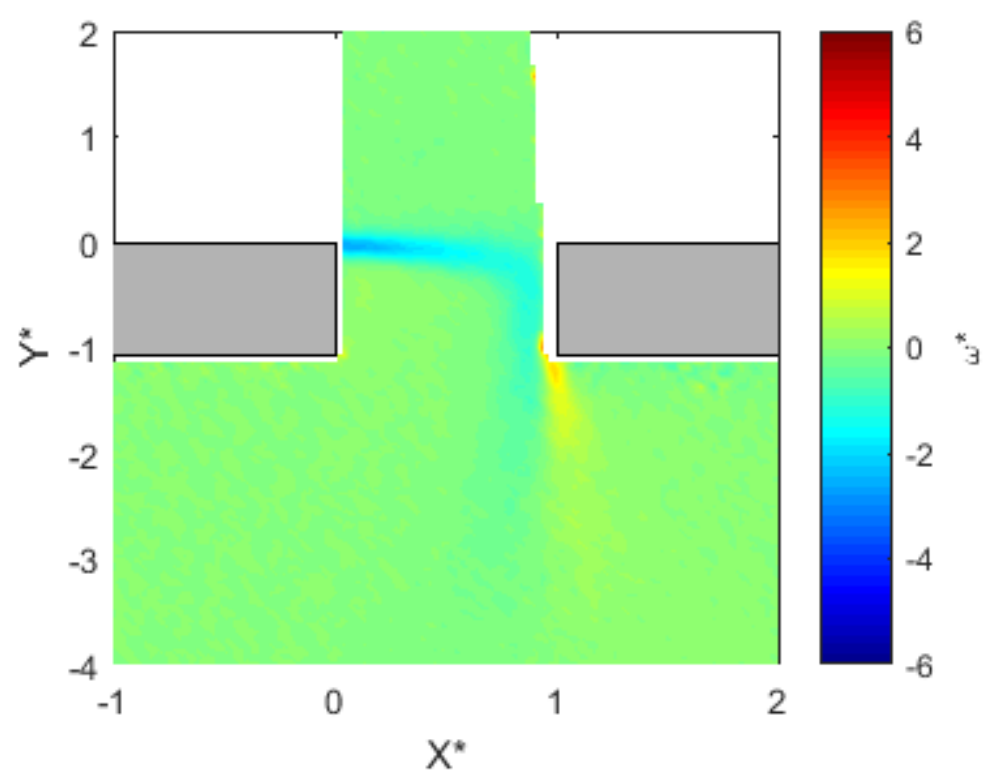

Figure 110: PIV results showing the average vorticity field at the 24th gap for a gap width of $6.35 \mathrm{~mm}$, an angle of incidence of 0 degrees, and a flow velocity of $20 \mathrm{~m} / \mathrm{s}$. 


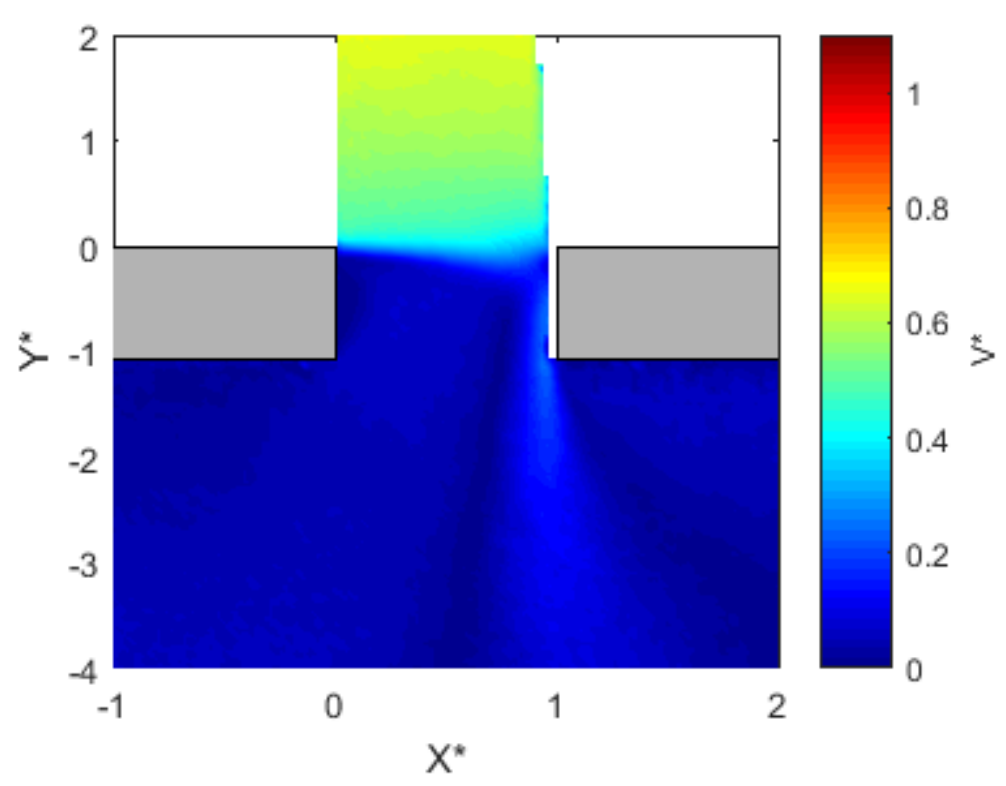

Figure 111: PIV results showing the average velocity field at the 24th gap for a gap width of $6.35 \mathrm{~mm}$, an angle of incidence of 0 degrees, and a flow velocity of $25 \mathrm{~m} / \mathrm{s}$.

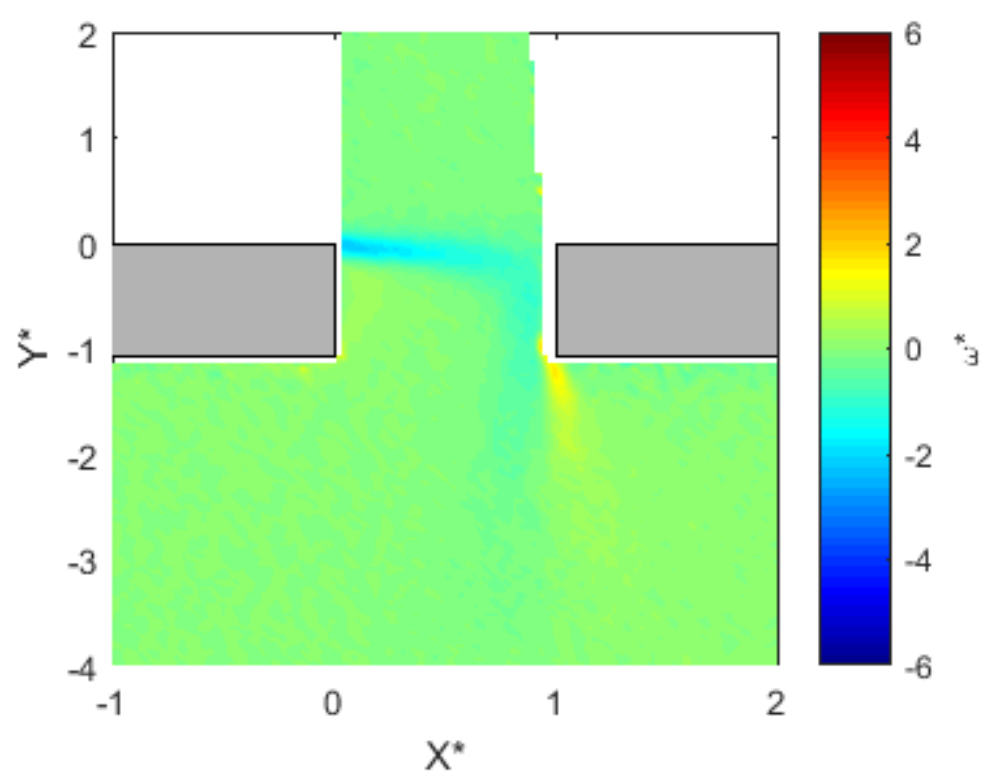

Figure 112: PIV results showing the average vorticity field at the 24th gap for a gap width of $6.35 \mathrm{~mm}$, an angle of incidence of 0 degrees, and a flow velocity of $25 \mathrm{~m} / \mathrm{s}$. 


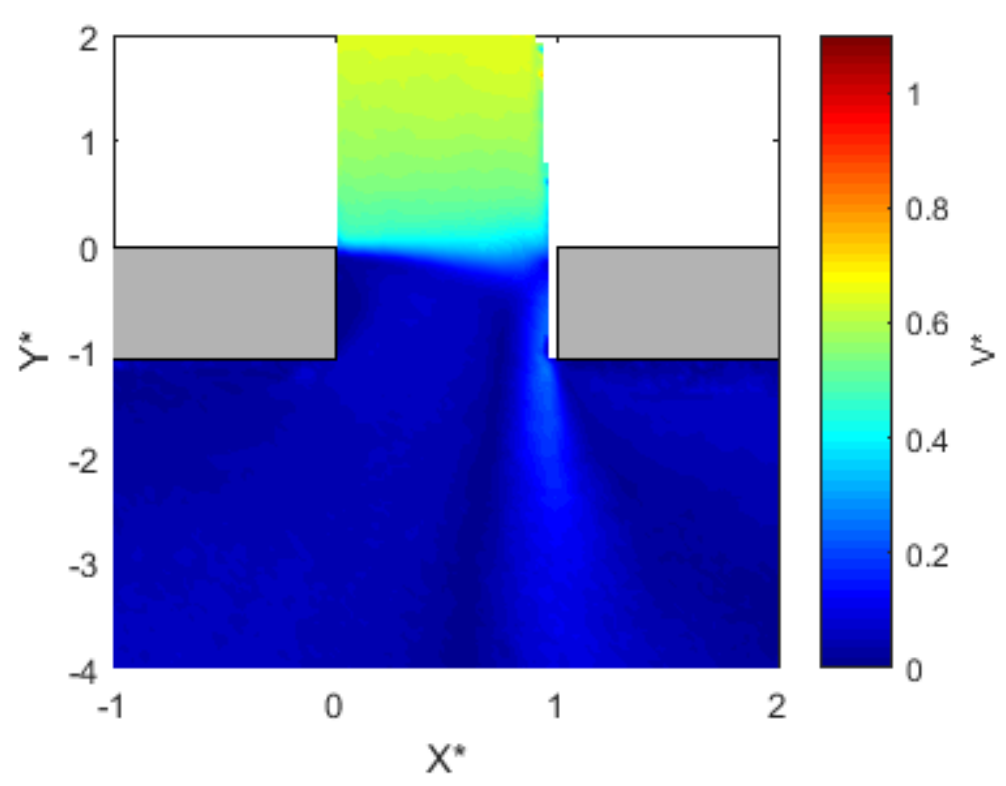

Figure 113: PIV results showing the average velocity field at the 24th gap for a gap width of $6.35 \mathrm{~mm}$, an angle of incidence of 0 degrees, and a flow velocity of $30 \mathrm{~m} / \mathrm{s}$.

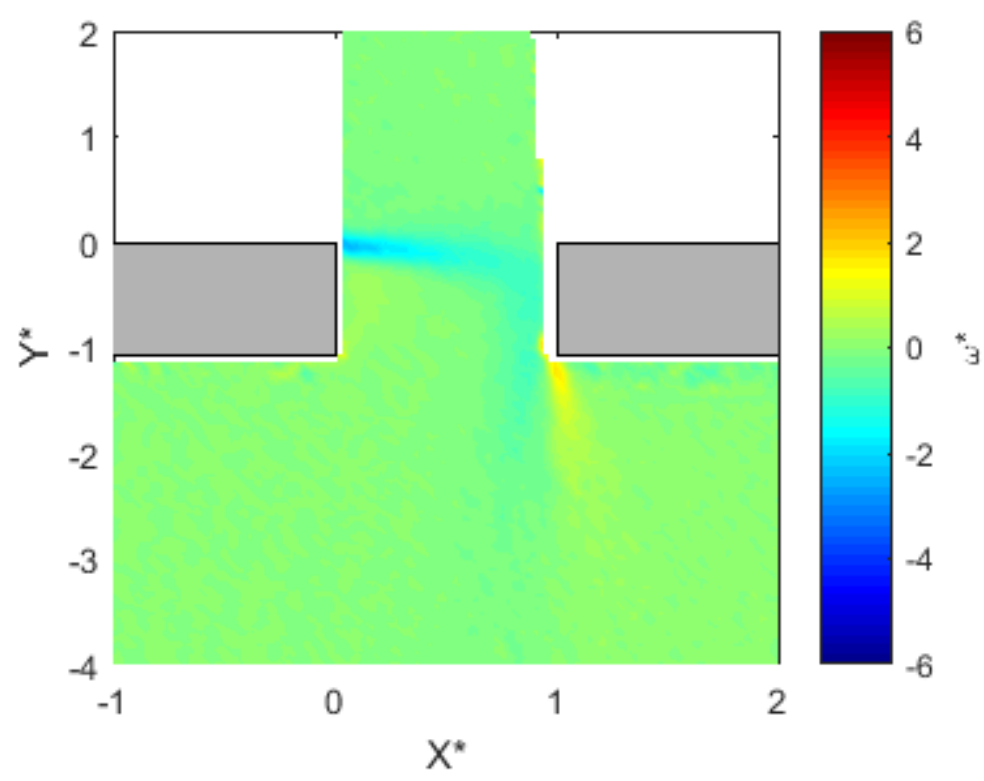

Figure 114: PIV results showing the average vorticity field at the 24th gap for a gap width of $6.35 \mathrm{~mm}$, an angle of incidence of 0 degrees, and a flow velocity of $30 \mathrm{~m} / \mathrm{s}$. 


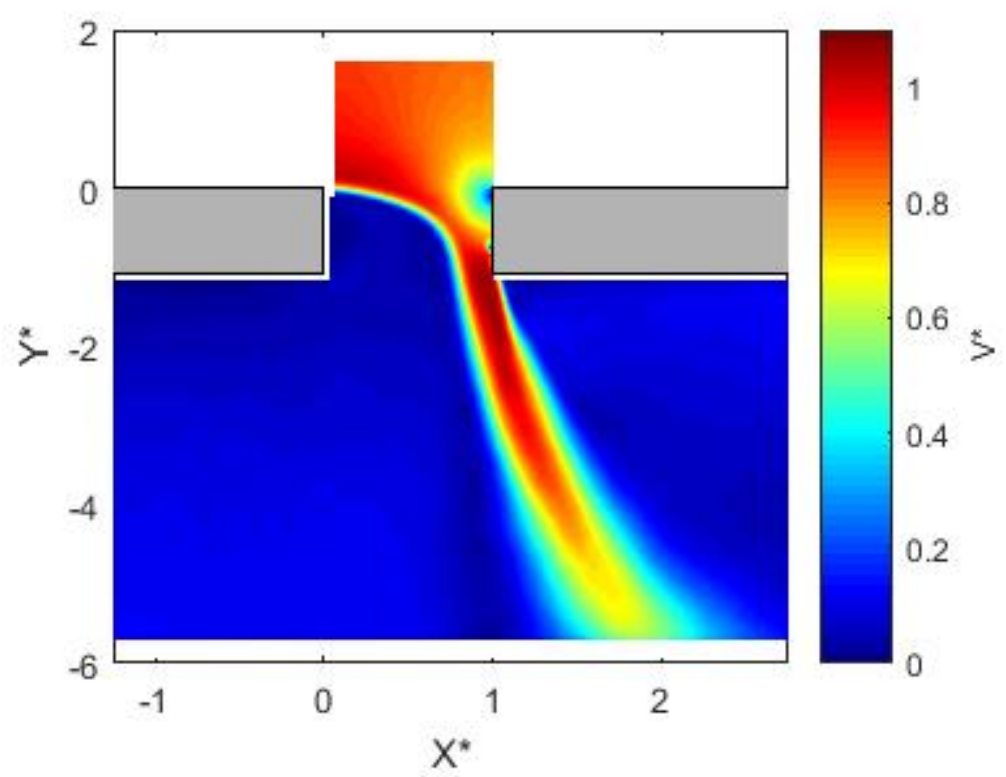

Figure 115: PIV results showing the average velocity field at the 24th gap for a gap width of $6.35 \mathrm{~mm}$, an angle of incidence of 35 degrees, and a flow velocity of $15 \mathrm{~m} / \mathrm{s}$.

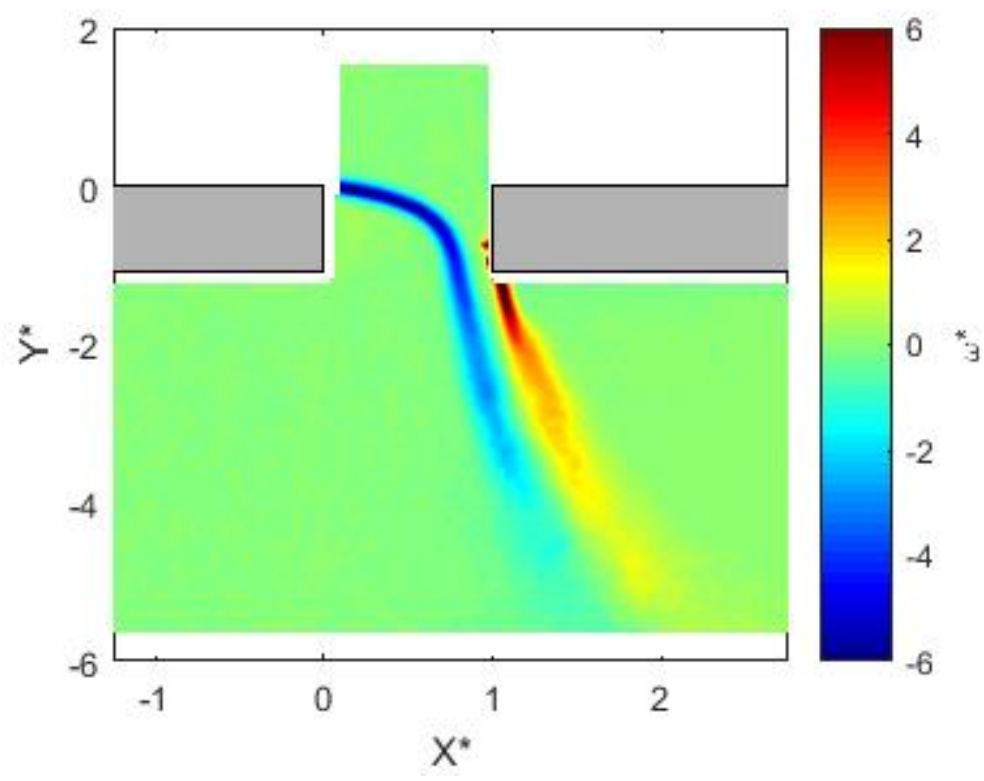

Figure 116: PIV results showing the average vorticity field at the 24th gap for a gap width of $6.35 \mathrm{~mm}$, an angle of incidence of 35 degrees, and a flow velocity of $15 \mathrm{~m} / \mathrm{s}$. 


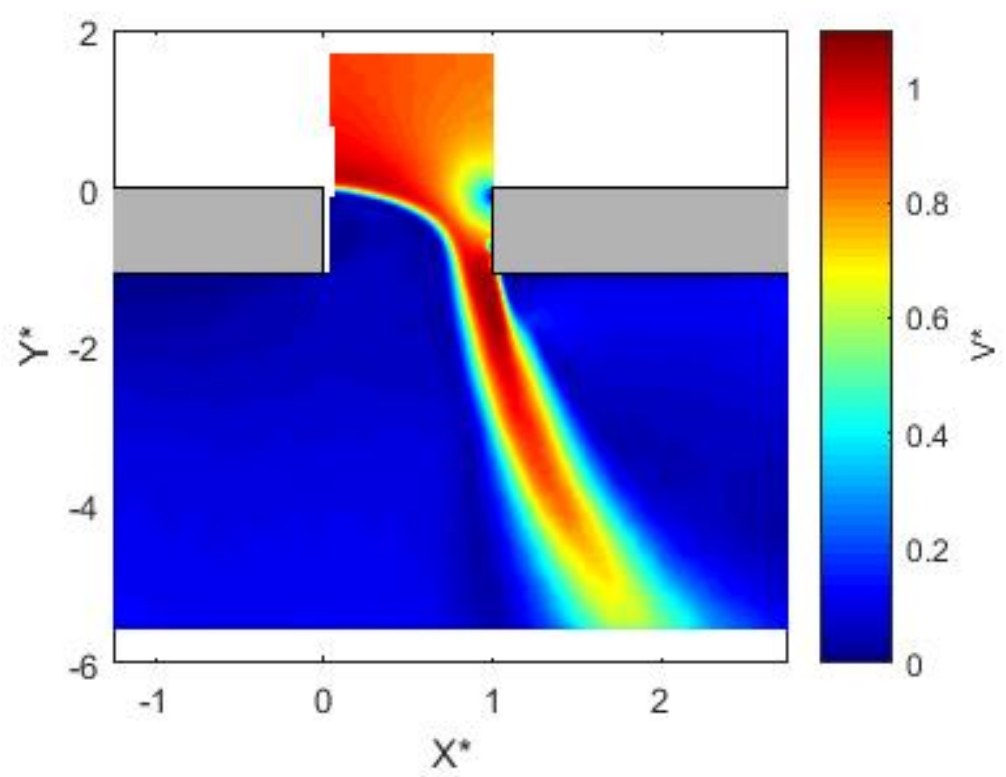

Figure 117: PIV results showing the average velocity field at the 24th gap for a gap width of $6.35 \mathrm{~mm}$, an angle of incidence of 35 degrees, and a flow velocity of $20 \mathrm{~m} / \mathrm{s}$.

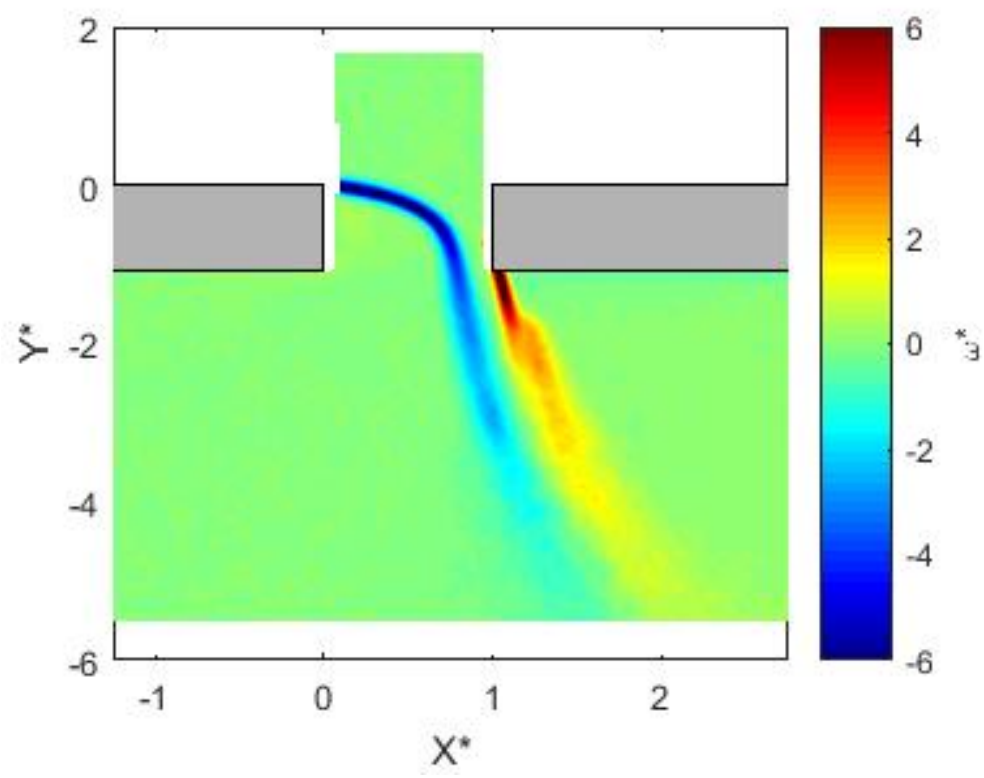

Figure 118: PIV results showing the average vorticity field at the 24th gap for a gap width of $6.35 \mathrm{~mm}$, an angle of incidence of 35 degrees, and a flow velocity of $20 \mathrm{~m} / \mathrm{s}$. 


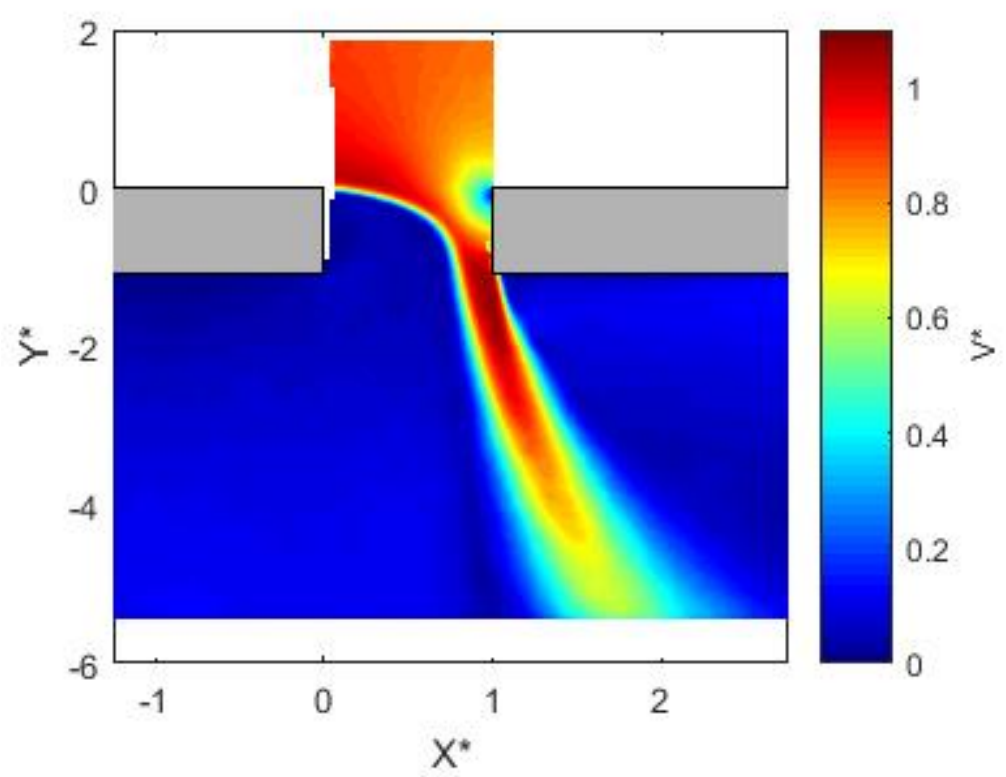

Figure 119: PIV results showing the average velocity field at the 24th gap for a gap width of $6.35 \mathrm{~mm}$, an angle of incidence of 35 degrees, and a flow velocity of $25 \mathrm{~m} / \mathrm{s}$.

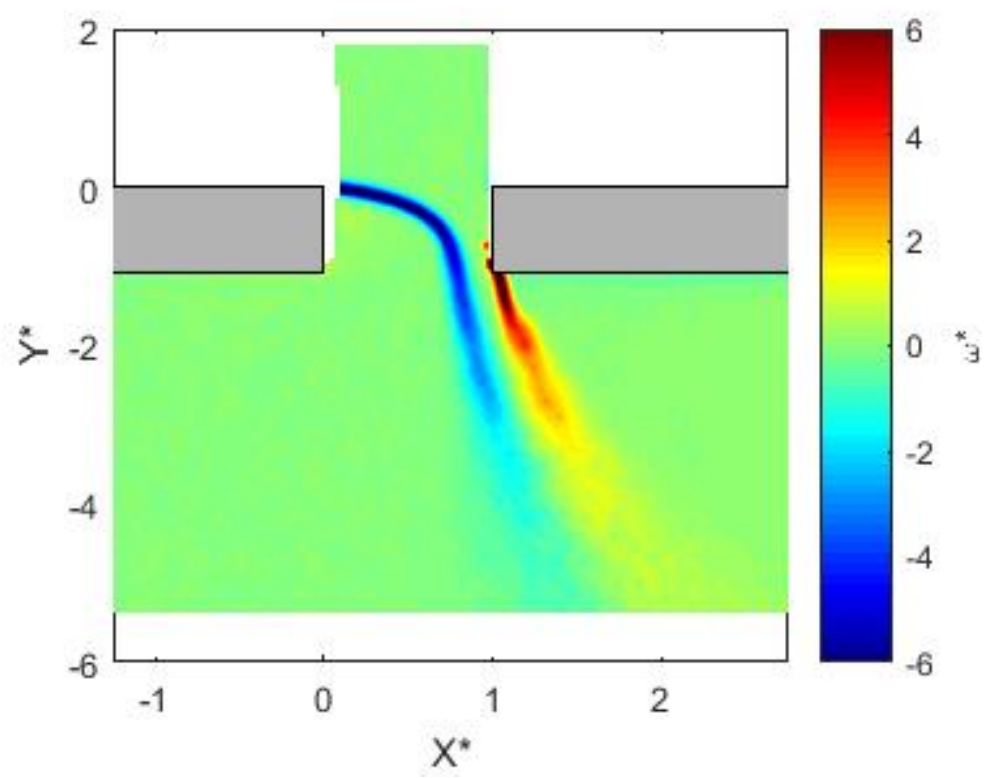

Figure 120: PIV results showing the average vorticity field at the 24th gap for a gap width of $6.35 \mathrm{~mm}$, an angle of incidence of 35 degrees, and a flow velocity of $25 \mathrm{~m} / \mathrm{s}$. 


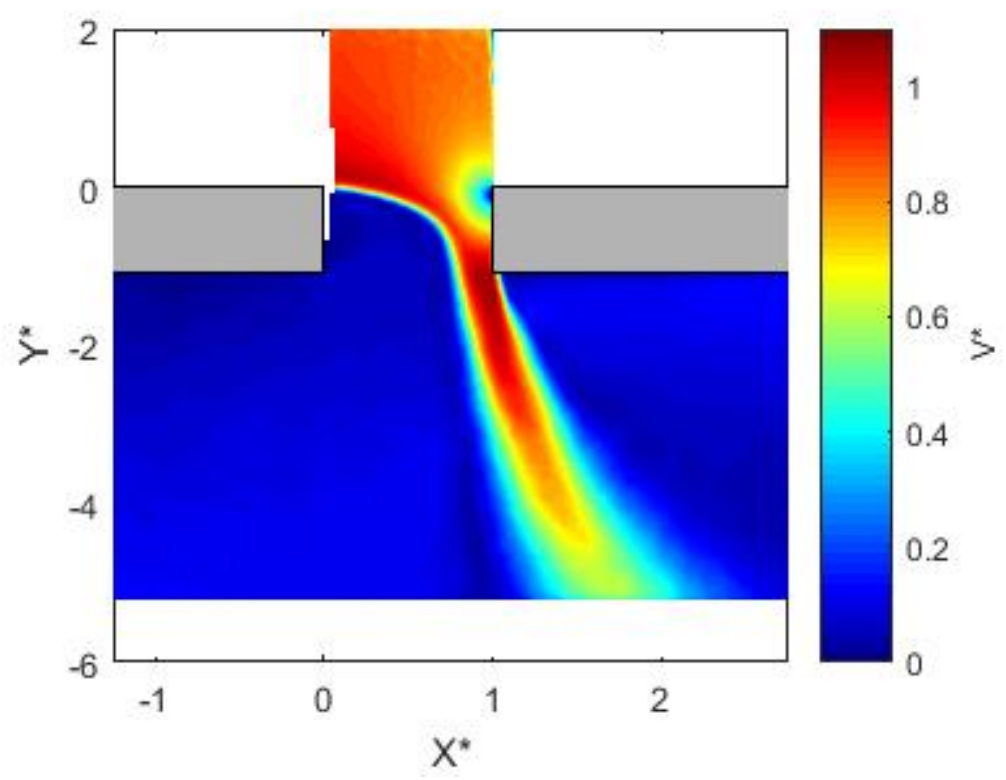

Figure 121: PIV results showing the average velocity field at the 24th gap for a gap width of $6.35 \mathrm{~mm}$, an angle of incidence of 35 degrees, and a flow velocity of $30 \mathrm{~m} / \mathrm{s}$.

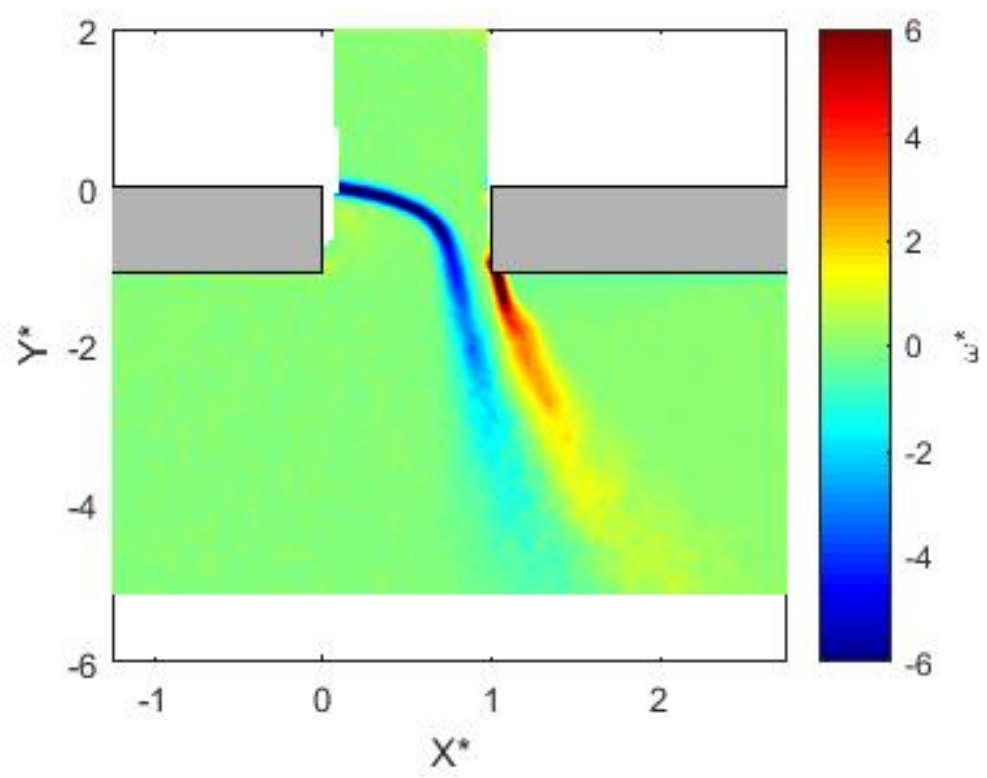

Figure 122: PIV results showing the average vorticity field at the 24th gap for a gap width of $6.35 \mathrm{~mm}$, an angle of incidence of 35 degrees, and a flow velocity of $30 \mathrm{~m} / \mathrm{s}$. 